\author{
UNIVERSIDAD NACIONAL DE LA PLATA \\ FACULTAD DE CIENCIAS EXACTAS \\ DEPARTAMENTO DE FÍSICA
}

\title{
DETERMINACIÓN DE LA ESTRUCTURA ISOMÉRICA DE COLORANTES FOTOISOMERIZABLES MEDIANTE EL ESTUDIO DE POLARIZACIÓN DE LA FLUORESCENCIA.
}

ROBERTO EMIR DI PAOLO

Trabajo de Tesis presentado para optar al título de Doctor en Física.

Director: Dr. Jorge O. Tocho

1997 
Esta Tesis fue realizada en el Centro de Investigaciones Ópticas (CIOp), dependiente de la Comisión de Investigaciones Científicas de la Provincia de Buenos Aires (CICBA). El período de trabajo estuvo comprendido entre los años 1990 y 1997. El mismo se realizó bajo la dirección del Dr. Jorge $\mathrm{O}$. Tocho, contando con la colaboración de los Drs. Lucía Scaffardi y Ricardo Duchowicz.

Parte de los resultados que se presentan a lo largo de esta Tesis, aparecen en las siguientes publicaciones:

"Dual laser excitation of a photochromic system: Application to DODCI".

Autores: R. Duchowicz, L. Scaffardi, R.E. Di Paolo y J.O. Tocho.

The Journal of Physical Chemistry; 96, 2501-2505, 1992.

"Spectroscopic properties of isomerizable cyanine dyes".

Autores: J.O. Tocho, R. Duchowicz, L. Scaffardi, G.M. Bilmes, R.E. Di Paolo y

M. Murphy. Trends in Physical Chemistry; 3, 31-47, 1992.

"Analysis of photoisomerizable dyes using laser absorption and fluorescence techniques".

Autores: R. Duchowicz, L. Scaffardi, R.E. Di Paolo y J.O. Tocho.

Laser Atomic and Molecular Physics (LAMP) Seminars, 1992, ICTP, Trieste, Italia.

"Photoisomerization dynamics and spectroscopy of the polymethine dye DTCI".

Autores: R.E. Di Paolo, L. Scaffardi, R. Duchowicz y G.M. Bilmes.

The Journal of Physical Chemistry; 99, 13796-13799, 1995.

"Polarization anisotropy applied to the determination of structural changes in the photoisomerization of DODCI".

Autores: R. E. Di Paolo and J. O. Tocho.

Chemical Physics; 206, 375-382, 1996.

"Rotational diffusion parameters of cyanine dyes determined by depolarization of luminiscence".

R.E. Di Paolo and J.O. Tocho.

Journal of Luminescence, 1997, (en prensa).

"Simultaneous absorption and fluorescence analysis of the DOCI cyanine dye".

L. Scaffardi, R. E. Di Paolo and R. Duchowicz.

J. of Photochemistry Photobiology, 1997, (en prensa). 


\section{AGRADECIMIENTOS}

Deseo expresar mi agradecimiento a todos aquellos que de una forma $u$ otra me han acompañado durante el periodo de realización de esta tesis, ya sea dirigiéndome en el trabajo o simplemente brindando su amistad y compartiendo gratos momentos. Creo sinceramente que todos han contribuido a crear un clima de trabajo cordial, donde siempre ha prevalecido la colaboración y la amistad. Por esta razón, a todo el Personal del CIOp mi más sincero agradecimiento por estos años vividos junto a ustedes.

En particular deseo agradecer al Dr. Jorge O. Tocho por la dirección de esta tesis. Su confianza y paciencia fueron fundamentales para que pudiera desarrollar mi trabajo con total libertad. No sólo me ha brindado la oportunidad de realizar mis tareas dentro de su grupo de investigación, sino que además quiero destacar su dedicación y apoyo en la concreción de esta tesis. Sin su orientación permanente la realización de la misma no hubiera sido posible.

Asimismo, quiero también agradecer muy especialmente a los Drs. Lucía Scaffardi y Ricardo Duchowicz, por su invalorable colaboración en diversos aspectos de mi trabajo. Con ellos he compartido muchas horas en el laboratorio, recibiendo su apoyo en todo momento.

A mis compañeros, en especial a Daniel Orzi y Miguel Murphy, con quienes he compartido tantos buenos momentos. Sus estímulos y colaboración me animaron siempre y fueron decisivos para alcanzar esta meta. A todos ellos mi más sincero agradecimiento.

A los Drs. Mario Garavaglia y Mario Gallardo, anterior y actual Director del Centro de Investigaciones Ópticas, les doy las gracias por su amable generosidad y acogida en esta Institución, que me ha hecho sentir desde el inicio parte integrante de la misma.

Deseo agradecer también a la Comisión de Investigaciones Científicas de la Provincia de Buenos Aires, por el otorgamiento de una Beca que me permitió durante la mayor parte de este tiempo contar con los recursos necesarios para dedicarme exclusivamente a la actividad de investigación, que se resume en esta tesis.

Para terminar, quisiera pedir al lector de esta tesis, le atribuya a quien me dirigió en la concreción de la misma todo lo bueno que pueda contener, dejando para mí los párrafos confusos y cuantas carencias pueda encontrar. 


\section{RESUMEN}

Determinar la estructura isomérica de un colorante en una solución líquida puede ser una tarea abordable mediante el estudio de la anisotropía de su emisión luminiscente. El cambio en la polarización de la luminiscencia tiene su origen en los movimientos rotacionales que experimenta la molécula de colorante en el seno del líquido. En la despolarización de su emisión está contenida la información referida a la forma estructural de la misma. Debido a que la difusión rotacional no sólo depende de esta característica de la molécula estudiada, sino también de factores tales como su volumen efectivo, la viscosidad del solvente o el tiempo de vida mismo del compuesto en su estado excitado, distinguir el efecto real de cada uno de ellos sobre el comportamiento observado requiere de un análisis detallado.

Los procesos fotofisicos de las cianinas estudiadas en esta tesis, colorantes polimetínicos que presentan fotoisomerización reversible, han sido analizados mediante el empleo de diversas técnicas. Dilucidar la estructura isomérica, tanto de las especies estables de estos compuestos como así también de aquellas especies que se generan bajo excitación luminosa (fotoisómeros), resulta ser por demás una tarea particularmente compleja cuando las características espectroscópicas entre las diferentes especies de un mismo compuesto son muy similares. En el estudio de las especies fotoisoméricas, la separación de la emisión de estas especies de aquella correspondiente a las especies normales, es necesaria para poder así seguir individualmente el comportamiento con la polarización de sus emisiones. El uso de técnicas láser de absorción y fluorescencia permitió obtener información de las especies fotoisoméricas de estas moléculas, analizando sus propiedades cinéticas y características espectroscópicas.

Con el fin de discernir las conformaciones isoméricas de las cianinas fotoisomerizables en solución, se implementó la técnica de polarización de la fluorescencia. Midiendo la anisotropía en estado estacionario de la fluorescencia que se genera bajo excitación luminosa, se pudieron calcular tiempos de difusión rotacional para las diferentes especies estudiadas. La asignación de determinadas estructuras isoméricas para estos compuestos, resulta de contrastar el resultado experimental de la anisotropía medida con modelos teóricos para la difusión rotacional de estas moléculas. Con el objeto de poder utilizar modelos moleculares más cercanos a las formas de las cianinas estudiadas, se representó las formas moleculares con elipsoides asimétricos, que permiten una aproximación más real a la geometría involucrada en el estudio de estas especies.

El uso de elipsoides asimétricos en el modelado de las estructuras moleculares y el análisis del comportamiento hidrodinámico de estos modelos con diferente condición de vínculo soluto-solvente, ha permitido ampliar la discusión a partir de los datos que se obtienen experimentalmente, posibilitando asignar distintas estructuras a los diferentes isómeros estudiados y dar en consecuencia una interpretación de los fenómenos observados. 
Del estudio realizado se concluye que la hidrodinámica "slip" es consistente con la anisotropía de la fluorescencia medida para las distintas especies de las cianinas. El similar comportamiento hidrodinámico de las especies más estables, se corresponde con la asignación de una estructura común a todas ellas. Una estructura en la que la cadena polimetínica de estos colorantes aparece totalmente extendida (todo-trans). En el caso particular del DODCI se ha podido descartar la estructura di-cis para la especie normal de esta cianina, dejando para su especie fotoisomérica alguna de las estructuras que resultan de rotar una parte de la molécula alrededor de un enlace de su cadena polimetínica. Una interacción ligeramente diferente entre las distintas especies de esta molécula y el solvente podría ser inferida de las medidas llevadas a cabo. El estudio fotofísico realizado sobre las cianinas permitió determinar entre otros parámetros, la baja eficiencia de fluorescencia de los fotoisómeros de las carbocianinas. Esta era una característica conocida de estos colorantes. De los resultados obtenidos y su posterior interpretación, la fricción dieléctrica resulta ser el fenómeno más indicado para acompañar a la fricción mecánica en un modelo hidrodinámico que dé cuenta de la difusión rotacional de las especies estudiadas. 


\section{ÍNDICE}

\section{CAPÍtULO 1}

\section{INTRODUCCIÓN GENERAL.}

I.1. INTRODUCCIÓN

1.2. DETERMINACIÓN DE LA ESTRUCTURA MOLECULAR DE LOS COLORANTES EN SOLUCIÓN

1.3. LA IMPORTANCIA DE LOS MÉTODOS TEÓRICOS EN EL ANÁLISIS DE LAS POSIBLES ESTRUCTURAS MOLECULARES

1.4. EL USO DE LA TÉCNICA DE POLARIZACIÓN DE LA LUMINISCENCIA

1.5. CONCLUSIÓN

\section{CApítulo 2}

PIROPIEDADES FÍSICAS DE LOS COLORANTES.

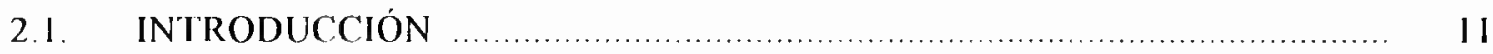

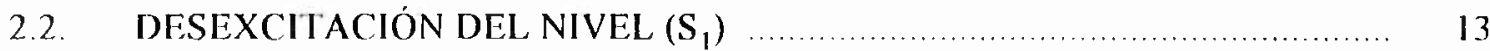

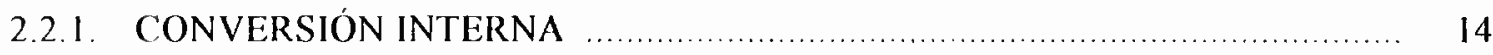

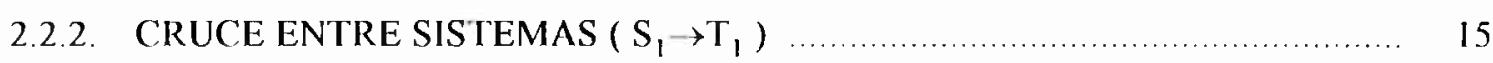

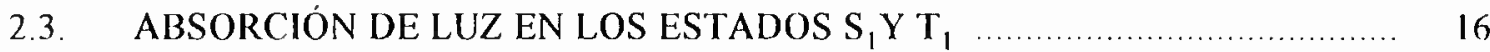

2.4. EFECTO DEL MEDIO SOBRE EL COMPORTAMIENTO DE

2.4.1. AGREGACIÓN DE LAS MOLÉCULAS DE COLORANTE _......................... 18

2.4.2. EFECTO DEL SOLVENTE SOBRE LAS TRANSICIONES

2.5. CÁlCULO DEL TIEMPO DE VIDA RADIATIVO DEL PRIMER

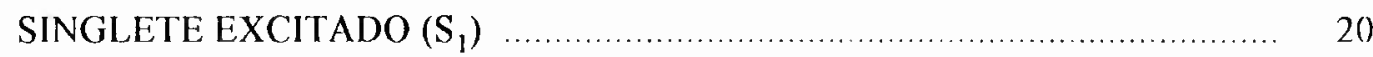

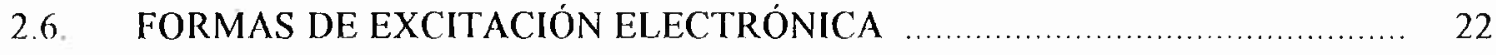

2.7 PROPIEDADES FÍSICAS DE LAS CIANINAS …................................ 23 


\section{CAPÍtulo 3}

POLARIZACIÓN DE LA FLUORESCENCIA.

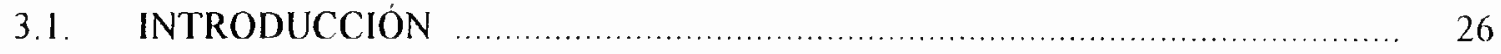

3.2. TEORÍA DE LA DESPOLARIZACIÓN DE LA FLUORESCENCIA _.............. 27

3.2.1. EXPRESIONES PARTICULARES DE LA ANISOTROPIA .......................... 33

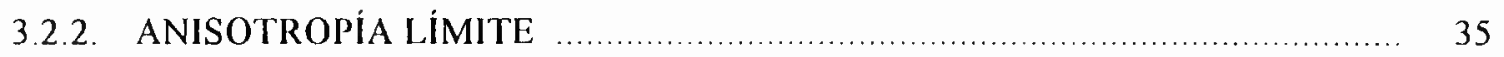

3.3. CÁLCULO DE LOS COEFICIENTES DE DIFUSIÓN ROTACIONAL ........... 37

3.3.1. COEFICIENTES DE DIFUSIÓN ROTACIONAL PARA UN ELIPSOIDE ASIMÉTRICO ............................................................. 39

3.3.2. MODELOS HIDRODINÁMICOS ( STICK - SLIP ) ................................. 41

\section{CAPÍTULO 4}

CARACTERIZACIÓN FOTOFÍSICA DE LAS CIANINAS ESTUDIADAS.

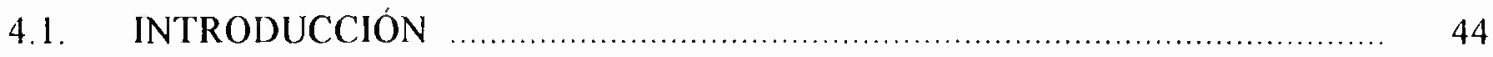

4.2. DESCRIPCIÓN DEL MODELO CINÉTICO _.......................................... 45

4.2.1. ECUACIONES DE BALANCE DE LAS POBLACIONES ......................... 46

4.3. TÉCNICAS UTILIZADAS EN LA DETERMINACIÓN DE LOS

PARÁMETROS CINÉTICOS Y ESPECTROSCÓPICOS DE

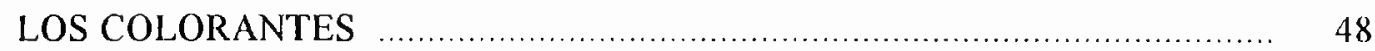

4.3.1. TÉCNICAS DE ABSORCIÓN …....................................................... 48

4.3.2. TÉCNICAS DE FLUORESCENCIA _................................................... 50

4.4. RESULTADOS DE LA APLICACIÓN DE ESTAS TÉCNICAS _.................... 52

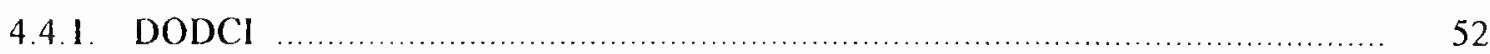

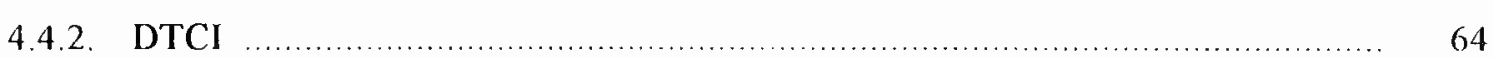

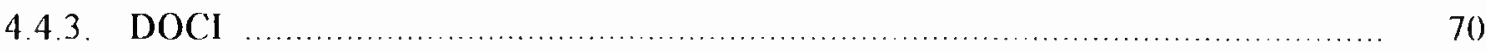

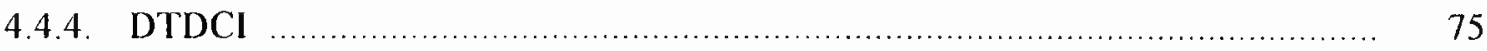

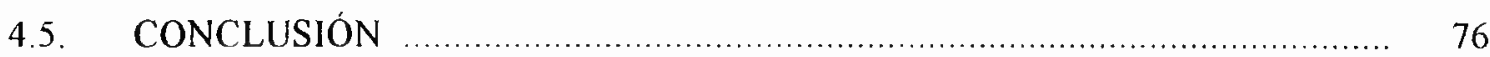

\section{CAPÍtulo 5}

DESARROLLO EXPERIMENTAL Y RESULTADOS DE ANISOTROPÍA.

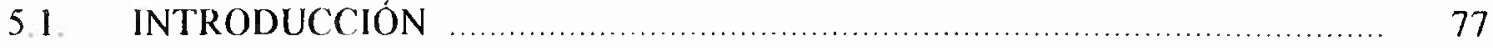

5.2. ESTUDIO DE LA DIFUSIÓN ROTACIONAL DEL DODCI ........................ 77

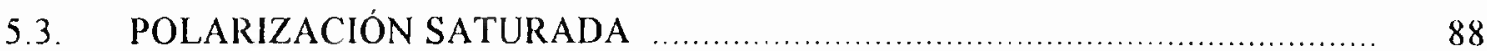


5.4. ESTUDIO DE LA DIFUSIÓN ROTACIONAL DE LAS ESPECIES

NORMALES

5.5. ANÁLISIS COMPARATIVO DE LAS CIANINAS ESTUDIADAS

CAPítulo 6

INTERPRETACIÓN DE LOS RESULTADOS.

CApítulo 7

CONCLUSIONES

120

\section{APÉNDICES}

A. EXPRESIÓN GENERAL DE LA ANISOTROPÍA

B. CONDICIÓN STICK-SLIP

C. DEPENDENCIA CON LA TEMPERATURA DEL TIEMPO

DE VIDA DE LA FLUORESCENCIA 


\section{CAPÍTULO 1: INTRODUCCIÓN GENERAL.}

\subsection{INTRODUCCIÓN.}

La absorción de radiación electromagnética por parte de moléculas orgánicas da lugar a una gran variedad de procesos, algunos de los cuales pueden interpretarse en términos de interacciones entre estados excitados. Los procesos fotofísicos, aquellos mecanismos de transformación de energía que no involucran cambios químicos, tienen su origen en muchos casos en estados singletes de vida corta (nanosegundos). En general el estudio de la cinética de formación y desactivación de estos estados requiere el empleo de altas potencias de excitación, constituyendo los láseres en estos casos una herramienta apropiada para tal efecto. La naturaleza de las transiciones no radiativas es un aspecto fundamental de la interacción radiación-materia, que representa de por sí una cuestión de interés más allá del sistema particular bajo estudio. El proceso de fotoisomerización de moléculas orgánicas en solución es un ejemplo en donde una parte de la energía absorbida por el compuesto durante su excitación, se usa para producir cambios estructurales reversibles

Los colorantes orgánicos jugaron un rol significativo en la física del láser. Sus características y propiedades han permitido el desarrollo de fuentes coherentes de muy alta eficiencia y óptimas posibilidades de sintonía. Los láseres basados en estos compuestos generan radiación en el rango de 400 a $1200 \mathrm{~nm}$. Además de ser sumamente adecuados como medio activo para la emisión láser, los colorantes orgánicos tuvieron un papel decisivo en algunos de los métodos desarrollados para la generación de pulsos láser ultracortos, de picosegundos y femtosegundos, actuando en calidad de absorbentes saturables en las técnicas de mode-locking pasivo [G.H.C.]. Un ejemplo que fuera de interés en esta línea de investigación lo constituye el caso del colorante 3,3'dietiloxadicarbocianina (DODCI). La combinación de un láser de Rodamina $6 \mathrm{G}$ como medio activo con esta cianina como absorbente saturable, en técnicas de mode locking pasivo, fue en su momento la única que permitió obtener pulsos de femtosegundos [Fork, Valdmanis]. La dinámica de funcionamiento de este tipo de sistemas no ha sido aún completamente esclarecida, debido a que la información que se tiene sobre los procesos fotofisicos que ocurren en el DODCI es todavía insuficiente, particularmente en lo que se refiere a los mecanismos de relajación del estado excitado del fotoisómero. Se sabe que en especial el mecanismo de fotoisomerización juega un rol decisivo en su eficiente utilización como absorbente saturable. Es por ello que un considerable esfuerzo puesto en el estudio de estos procesos, ha acompañado el desarrollo de las técnicas de generación de pulsos ultracortos.

Los procesos de fotoisomerización son característicos de muchos compuestos orgánicos y ocurren también en fotorreceptores biológicos, donde la radiación absorbida es 
empleada para transmitir información. Como ejemplo puede mencionarse que la fotoisomerización cis-trans constituye la base de las transformaciones de retinal, que juega un rol importante en el proceso de la visión. Estos sistemas, que en general son complejos, tienen estructuras formadas por cadenas polimetínicas similares a las que poseen las cianinas, colorantes orgánicos sintéticos. Sus propiedades fotofísicas y espectroscópicas las han convertido actualmente en buenos modelos dentro del área de la fotobiología, de moléculas biológicas que se usan para detectar luz, traducir señales luminosas, etc. Las cianinas constituyen también una familia de colorantes polimetínicos de gran aplicación en biofisica como sondas fluorescentes de las propiedades de biomembranas [Minch, Nakashima, Grieser]

Otra propiedad de los colorantes polimetínicos la constituye su capacidad para volver sensible a la luz visible compuestos basados en haluros de plata. Su facilidad para la agregación y adsorción sobre estos últimos ha sido determinante para su utilización como sensibilizadores en la industria fotográfica del color [Sturmer].

La estructura de las cianinas en solución no se conoce muy bien. En general si no tienen sustituyentes en la cadena serían todo-trans. (Esta puede verse en la página 23). Pero distintos isómeros pueden existir por rotaciones alrededor de los enlaces de la cadena polimetínica. En vista de las propiedades expuestas de estos colorantes, y su participación ya sea directa o indirecta en diversos procesos que involucran a especies isoméricas de estos compuestos, queda implícita la importancia de un estudio sobre las características estructurales de los colorantes polimetínicos. El análisis de los movimientos de difusión rotacional durante el tiempo de vida de moléculas excitadas, brinda un método apropiado para caracterizar la estructura isomérica de estas moléculas. En lo que sigue, se discutirá la conveniencia o no de este y otros métodos para alcanzar tal fin

\subsection{DETERMINACIÓN DE LA ESTRUCTURA MOLECULAR DE LOS COLORANTES EN SOLUCIÓN}

A pesar de la batería de métodos teóricos y experimentales aplicados a la identificación de las especies isoméricas de colorantes, no ha sido posible aún establecer sin ambigüedades la estructura de los mismos en solución. Dentro de los métodos experimentales que han sido utilizados en el estudio conformacional de las cianinas, el análisis de difracción de rayos $\mathrm{X}$ requiere condiciones especiales, como ser la preparación de muestras cristalinas, que no siempre pueden realizarse. Además, la estructura de las especies estudiadas en matriz cristalina puede ser muy diferentes de aquella que adopten en fase líquida. 
En la figura 1.1 pueden verse los contomos de densidad electrónica, obtenidos a partir de los datos de difracción de rayos $X$, de la fialocianina cuya representación estructural se indica abajo.
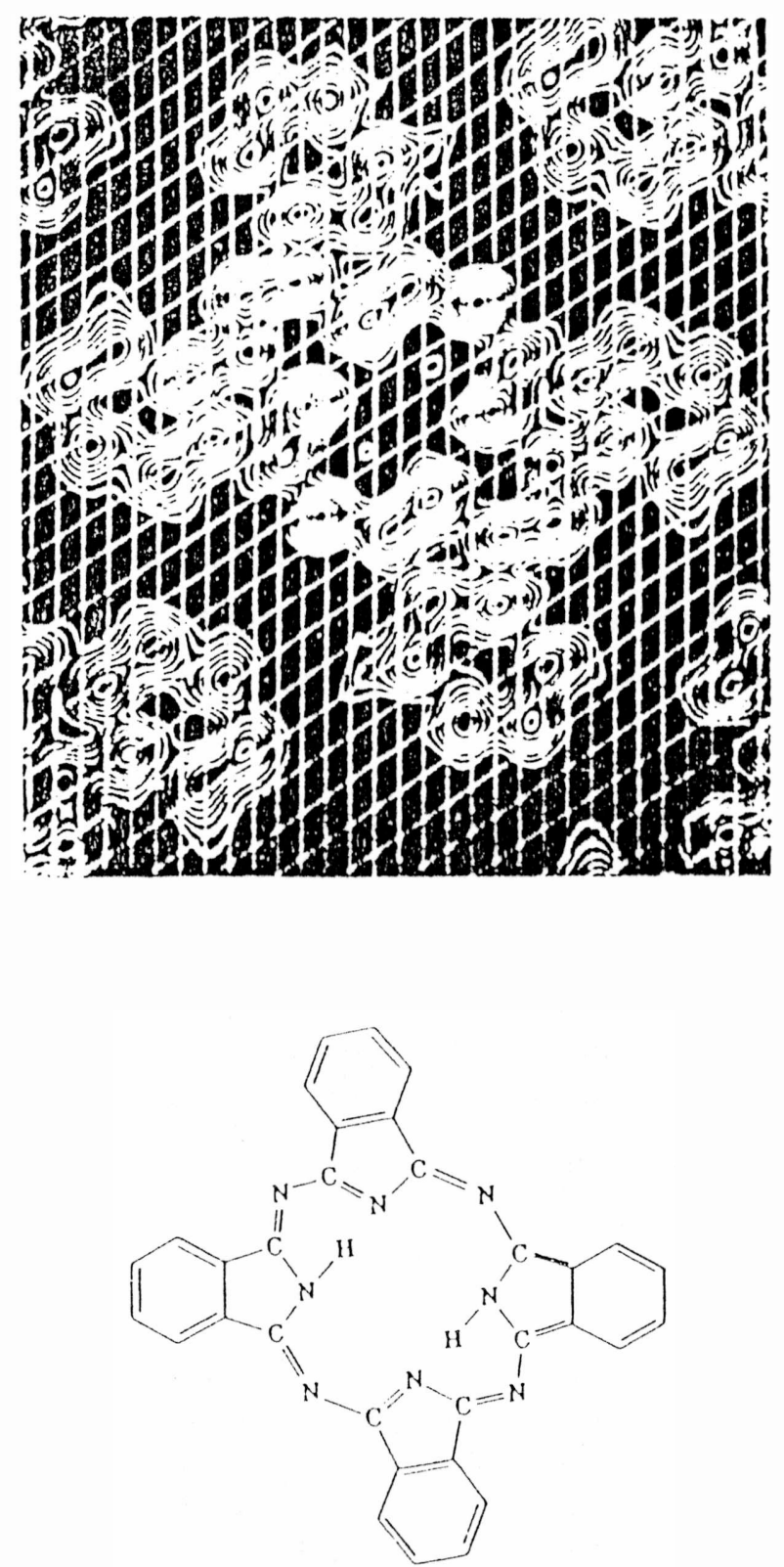

Figura I.I

Datos de difracción de ayos $X$ para una scrie de cianinas, pucden ser encontradas en el review de Smith. El análisis con rayos X de la molécula dietiltiacarbocianina (DTCI), 
no sustituida en su cadena polimetínica, indica que estas especies adoptan una estructura completamente todo-trans y son casi planas [Wheatley]. Acorde a los diferentes datos, los ángulos entre los planos de los núcleos heterocíclicos y el plano de los restantes átomos del cromóforo, están en el orden de unos pocos grados.

El análisis de los espectros electrónicos, que constituyen la característica más importante de los colorantes, no resulta ser el método más conveniente en esta instancia. La información que se infiere de ellos contiene la acción de diversos factores que dificulta la separación de los mismos. Las bandas de absorción de carbocianinas $\beta$ sustituidas en solución, se desdoblan a baja temperatura en dos bandas muy próximas entre sí [West]. La intensidad de cada banda aumenta excitando la banda vecina con radiación en la longitud de onda de su máximo de absorción. Cuando cesa la irradiación el sistema vuelve a su estado inicial de equilibrio. Los datos experimentales se pueden interpretar asumiendo un proceso de fotoisomerización trans $\rightarrow$ cis. Pero dado que la interacción del sustituyente con los núcleos terminales haría aumentar la energía del estado base de la forma todo-trans del compuesto, volviéndola comparable a la de la conformación cis, la dirección de este proceso podría no ser así. Puede verse también que el equilibrio entre las especies depende del solvente. El efecto del solvente sobre los espectros es otro de los inconvenientes en el presente análisis

Un método más efectivo para la determinación de estructuras isoméricas de colorantes polimetínicos, lo constituye la resonancia magnética nuclear (NMR). Este método, aplicado en principio a estudios en sólidos, puede extenderse a fase líquida con una elección adecuada del solvente. El análisis de la forma y el número de señales en los espectros NMR de carbocianinas $\beta$-sustituidas, hace posible la determinación de la estructura geométrica de los confórmeros presentes y posibilita estimar cuantitativamente la posición del equilibrio conformacional [Henrichs]. Se ha establecido, por ejemplo, que un incremento en el bulk efectivo del sustituyente en la posición $\beta$, promueve la aparición del isómero cis. Datos preliminares de NMR mostrarían que la forma estable de la molécula dietiloxacarbocianina (DOCI), al igual que la correspondiente tiacarbocianina, es una estructura todo-trans plana.

En cuanto a las dicarbocianinas, se sabe que el catión tiadicarbocianina (DTDCI) existe en fase sólida en conformación todo-trans y es una estructura casi plana. La NMR de dicarbocianinas sustituidas indica que estos compuestos existirian en solución exclusivamente en conformación todo-trans, favoreciendo la máxima superposición de los orbitales y la consecuente deslocalización de los electrones $\pi$. Este resultado contrasta con uno previo, que habla de la separación por cromatografia de tres isómeros de la tiatricarbocianina sobre carbonato de calcio a temperatura ambiente [Zechmeister]. 
Asimismo la información que se tiene para DODCI (dietiloxadicarbocianina) acerca de este punto, resulta indirecta y contradictoria. En un estudio de difusión rotacional, se sugirió que la fotoisomerización de este colorante consiste en la rotación alrededor de dos enlaces de la cadena polimetínica, llevando la molécula de una estructura compacta di-cis (isómero estable) a una todo-trans (fotoisómero) [Fleming, \#49].

El problema de determinar cual de las posibles fotoisomerizaciones tiene lugar realmente en estas moléculas, no puede establecerse con certeza sobre la base de la evidencia experimental existente

\subsection{LA IMPORTANCIA DE LOS MÉTODOS TEÓRICOS EN EL ANÁLISIS DE LAS POSIBLES ESTRUCTURAS MOLECULARES.}

El comportamiento observado experimentalmente de los espectros de absorción de polienos conjugados y sus derivados, pudo ser reproducido en el pasado, al menos en forma cualitativa, con cálculos teóricos sobre distintos isómeros de estos compuestos. De acuerdo con la teoría [Mulliken], la conformación de las moléculas afecta la distribución de intensidad y la intensidad total del espectro de absorción. Cuanto más alargada es la molécula, mayor la intensidad total y mayor la intensidad correspondiente a la transición en longitudes de onda más larga. Los espectros calculados usando la aproximación de Hückel de orbitales moleculares (MO), permiten reflejar las diferencias observadas en el comportamiento de distintos isómeros de estos compuestos. Experimentalmente se ve que al aumentar el número de dobles enlaces conjugados, se produce un corrimiento de los espectros hacia longitudes de onda más largas, con un aumento en su intensidad

De los cálculos se advierte que para la forma todo-trans de las moléculas, la transición de absorción más fuerte está polarizada aproximadamente a lo largo del eje mayor de la molécula, dando origen así a una alta polarizabilidad de estos compuestos en aquella dirección. Otras transiciones mucho más débiles, en longitudes de onda más corta, aparecen polarizadas perpendicularmente a este eje

Un hecho característico del espectro electrónico de los isómeros cis es la absorción apreciable en longitudes de onda corta (UV), conocida como pico cis [Kolesnikov]. Esta está completamente ausente en los espectros de isómeros trans, como consecuencia de resultar una transición no permitida para esta estructura. La isomerización trans-cis levantaría entonces esta prohibición, permitiendo distinguir a la especie todo-trans del resto de los posibles isómeros.

Si bien la teoría falla en el sentido cuantitativo, esta predice cualitativamente bien el corrimiento hacia longitudes de onda más largas, con un aumento en la intensidad de las 
transiciones, al aumentar el número de doble enlaces conjugados en la cadena polimetínica.

El modelo cinético para la fotoisomerización de cianinas propuesto por Rulliere, resultó ser el más apropiado para explicar los datos fotofísicos y fotoquímicos reportados a la fecha [Negri]. De acuerdo con este modelo, el ángulo de torsión alrededor de un enlace de la cadena es la coordenada de reacción. Desde un estado intermedio torsionado, la molécula relaja al estado fundamental de alguna de sus especies. Sobre la base de su modelo de diagramas de energía potencial para los niveles electrónicos de cianinas pentametinicas, simples consideraciones basadas en la correlación Hückel MO, complementadas por cálculos CS-INDO/CI [Ponterini], muestran claramente que una doble isomerización simultánea en estos compuestos es muy improbable. Esta se caracteriza por una barrera mucho más alta que aquella correspondiente a una sola isomerización.

Diferentes aproximaciones teóricas han sido empleadas en los últimos tiempos, para determinar las estructuras más estables de los distintos isómeros e identificar de esta manera las coordenadas de isomerización para estos compuestos [Awad]. Así por ejemplo, en la optimización del procedimiento de modelado molecular, alterando sistemáticamente los ángulos de torsión de los enlaces, se busca para cada una de las estructuras aquella con la energía asociada más baja. Para ello se usan programas de cálculo semiempíricos (MOPAC 5.0 y el AM1). Las conformaciones todo-trans generalmente son las que tienen el calor de formación más pequeño [Churio, \#98].

\subsection{EL USO DE LA TÉCNICA DE POLARIZACIÓN DE LA LUMINISCENCIA}

La espectroscopia de la fluorescencia polarizada no ha llegado a ser aún un método rutinario de análisis como lo es hoy la espectroscopia de absorción y la de emisión. Los métodos fluorescentes están encontrando una aplicación creciente en los campos de la química, la fotofisica y la bioquímica, en vista del hecho que muchos procesos fisicos naturales son muy rápidos y ocurren en tiempos del orden de los nano y picosegundos. Se trata básicamente de procesos de relajación, tales como decaimiento de la fluorescencia de moléculas orgánicas en solución, la transferencia de energía de excitación intra e intermolecular y el cambio de la anisotropía de la emisión debido a movimientos rotacionales de las moléculas durante el tiempo que permanecen excitadas.

La excitación de moléculas orgánicas luminiscentes en solución, afecta la distribución isotrópica de los momentos dipolares de transición de estas moléculas. Como resultado de esta excitación la fluorescencia anisotrópica resultante, esto es el grado de 
polarización de esta luminiscencia, puede ser observada. La anisotropía de la fluorescencia constituye una fuente valiosa de información acerca de los procesos fotofisicos que tienen lugar en moléculas complejas. A partir de ella pueden extraerse conclusiones sobre transferencia de energía entre moléculas en sistemas condensados, dirección de momentos de transición en absorción y emisión, estructura molecular y cambios conformacionales, propiedades tales como forma y dimensiones de las moléculas, momentos dipolares eléctricos en estados excitados, etc.

Los métodos de espectroscopia fluorescente juegan un rol clave en la investigación de las propiedades dinámicas de ácidos nucleicos, proteínas y membranas biológicas. De particular interés son aquellos que se basan en el estudio de la despolarización de la luminiscencia de pruebas fluorescentes naturales o bien de sondas que son introducidas artificialmente. Estas permiten seguir procesos de difusión rotacional rápidos, con constantes de tiempo que dependen de la sonda y la técnica experimental empleada. La introducción de técnicas de nano y picosengundos ha permitido alcanzar una mejora importante en los estudios de polarización.

La iluminación de la muestra con un haz de luz polarizado linealmente es el ejemplo típico de la excitación anisotrópica de la fluorescencia. En estos casos, el campo de radiación fotoluminiscente muestra simetría axial. La dirección del vector eléctrico de la luz de excitación constituye este eje de simetría. Cuando se usa luz natural para excitar la muestra, el eje de simetría lo determina la dirección perpendicular al plano de vibración del campo eléctrico del haz de excitación.

Existe una serie de consideraciones que se adoptan cuando se trata la anisotropía de la fotoluminiscencia. En primer lugar se supone que no existe relación de fase entre la luz de excitación y la luminiscencia que emiten las moléculas excitadas. Dado que el proceso de absorción ocurre en femtosegundos, mientras que los tiempos de vida de las moléculas luminiscentes son del orden de los nanosegundos, la absorción y la emisión son procesos bien separados temporalmente como para que se establezca tal relación. La suposición es válida siempre que la intensidad de excitación sea suficientemente baja como para poder despreciar la emisión inducida. Otra de las hipótesis de trabajo, es que las moléculas que emiten son fuentes incoherentes. Es decir, que no existe relación de fase entre la luz emitida por las diferentes moléculas. En este caso, las intensidades de las componentes individuales pueden simplemente ser sumadas. En cuanto a la dirección del momento de transición molecular responsable de la emisión, se entiende que este depende del método de excitación y sólo depende de los estados moleculares entre los cuales ocurre la transición.

Debido a la direccionalidad de los momentos de transición electrónica de las moléculas, un haz de luz crea en la muestra un conjunto anisotrópico de moléculas excitadas. Dado que existe una dependencia orientacional de la probabilidad de absorción, inmediatamente después de la excitación se observará una orientación preferencial de las 
moléculas excitadas. Es a este fenómeno al que se lo conoce como fotoselección [Albrecht] De acuerdo con el mismo, los momentos de transición de las moléculas excitadas tendrán una distribución particular alrededor de una dirección vinculada con el haz de excitación, y el resto de las moléculas (aquellas que no fueron excitadas) una distribución complementaria

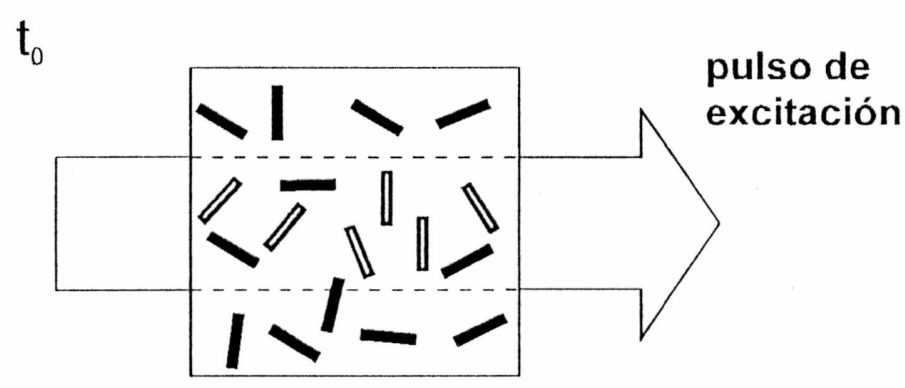

moléculas

en su estado

fundamental

$\| \begin{aligned} & \text { moléculas } \\ & \text { excitadas }\end{aligned}$

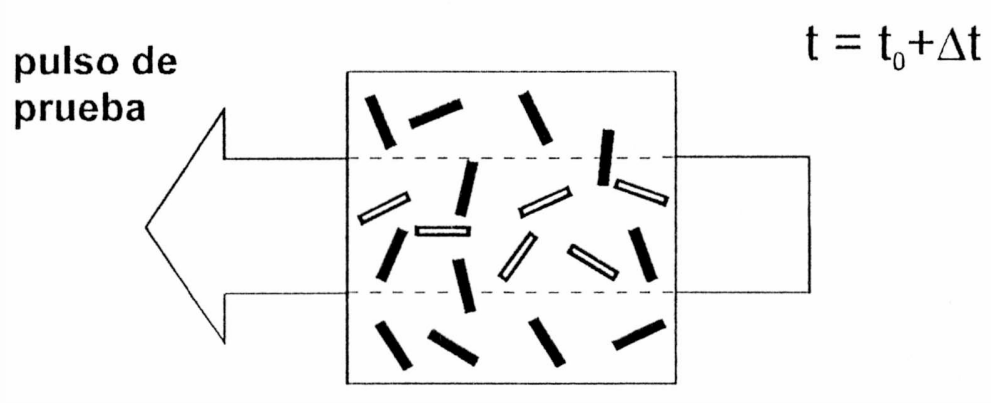

Figura 1.2. El pulso de excilación crea una distribución particular de moléculas excitadas. Por relajación orientacional el pulso de prueba encuentra una distribución diferente, tanto de las moléculas excitadas como de las que no lo están.

En un líquido las moléculas continúan moviéndose por difusión rotacional (ver Fig. 1.2) Luego, cuando un pulso de prueba alcanza la muestra en un tiempo $\Delta$ t más tarde, las moléculas excitadas anteriormente $\left(\mathrm{en}_{0}\right.$ ) han rotado, alterando la posición de sus momentos de transición respecto de la polarización de referencia, o habrán relajado al estado fundamental. Por otra parte, habrá moléculas que no fueron excitadas en un principio y que ahora estarán alineadas con el campo de excitación. Evidentemente la difusión rotacional, lo mismo que el proceso cinético que vuelve las moléculas a su estado 
fundamental, contribuye a la relajación toda de la transmisión. Este fotodicroísmo inducido, como suele llamársele, es observado aún en matrices viscosas [Lombardi]. La luz polarizada y la difusión rotacional contribuyen virtualmente en cualquier experimento de transmisión transiente de colorantes en solución. El primer experimento de picosegundos sobre fotodicroísmo inducido, fue realizado por Eisenthal y Drexhage.

En las medidas del decaimiento de la fluorescencia basta con una elección adecuada de las direcciones de polarización de la excitación y de la emisión, para evitar el efecto de la difusión rotacional sobre las medidas. De la misma manera, siempre es posible medir la transmisión transiente salvando este problema [Lessing, 1979], con una elección adecuada del ángulo entre las direcciones de polarización de los haces de prueba y blanqueo, que cancele el efecto de la fotoselección y la relajación orientacional.

\subsection{CONCLUSIÓN.}

Tal como ya fuera señalado, la similitud estructural de las cianinas con muchos pigmentos biológicos, las convierte en buenos modelos de sistemas fotobiológicos más complejos. Las cianinas sufren una isomerización reversible a través de su cadena polimetínica cuando son excitadas. Para algunas carbocianinas no sustituídas este proceso ocurre vía el estado singlete excitado, compitiendo entonces con la fluorescencia. A pesar de ser la fotoisomerización de estos compuestos un proceso constantemente investigado, la evidencia experimental sobre la conformación de los isómeros es escasa. Los evidentes avances logrados con la mejora de las técnicas espectroscópicas y la posibilidad de sintetizar nuevos compuestos, no han facilitado la identificación de los confórmeros, que sigue siendo en estos días una tarea compleja.

En la búsqueda de una solución al problema de dilucidar qué especies intervienen en la fotoisomerización de estos compuestos, esto es, cual es la forma estable de estas moléculas y cual aquella que se genera bajo excitación luminosa, se decidió como primer objetivo la implementación de la técnica de polarización de la fluorescencia, para su aplicación en la determinación de las estructuras isoméricas de cianinas en solución. El problema ha sido tratado sobre la base de un análisis previo de los mecanismos de fotoisomerización, que tienen lugar cuando se excita a estas moléculas con diferentes fluencias de radiación. El proceso de fotoisomerización de cianinas simétricas ha sido estudiado combinando técnicas de absorción y fluorescencia, buscando en particular caracterizar las especies fotoisoméricas de estos compuestos.

Las cianinas elegidas para llevar adelante este trabajo han sido cuatro. El DODCI ya mencionado y otras tres cianinas que se diferencian de la primera por el largo de la cadena 
polimetínica y/o los núcleos heterocíclicos presentes. Esta elección se basa en el conocimiento previo de ciertas propiedades de estos compuestos que posibilitan avanzar en su estudio estructural y en segundo lugar el interés por ver el efecto de las características mencionadas sobre su comportamiento fotoisomérico. En virtud de hacer el estudio en estado estacionario, se requiere conocer el tiempo de decaimiento de la fluorescencia de los compuestos elegidos, para poder así a partir de la anisotropía estudiada, inferir información sobre otros aspectos como ser la estructura isomérica de estos compuestos

Las cuatro moléculas estudiadas son: 3,3'-dietiloxadicarbocianina (DODCI), 3,3'dietiloxacarbocianina (DOCI), 3,3'-dietiltiadicarbocianina (DTDCI), y 3,3'-dietiltiacarbocianina (DTCI). La anisotropía de la fluorescencia de estos compuestos es analizada sobre la base de aspectos teóricos que han sido desarrollados para permitir extender la discusión al caso de modelos geométricos más próximos al de estas moléculas. Esto posibilitó interpretar los resultados experimentales en términos de rotores asimétricos, buscando determinar volúmenes moleculares efectivos y decidir entre diferentes condiciones de contorno hidrodinámicas. 


\section{CAPÍTUlO 2: PROPIEDADES FísICAS dE lOS COLORANTES.}

\subsection{INTRODUCCIÓN}

Los colorantes orgánicos se caracterizan por tener una fuerte banda de absorción en la región visible del espectro electromagnético. Tal propiedad se encuentra sólo en compuestos orgánicos que contienen un sistema extendido de enlaces conjugados, esto es, alternando enlaces simples y dobles.

La configuración electrónica más baja de estas moléculas es un estado singlete. Los electrones ocupan de a pares los niveles más bajos. Cuando la molécula es excitada y un electrón salta a un estado superior, el spin total del sistema de fermiones podría seguir siendo cero o pasar a ser uno (estado triplete). La energía del sistema en este último estado cs algo menor que en el estado singlete excitado.

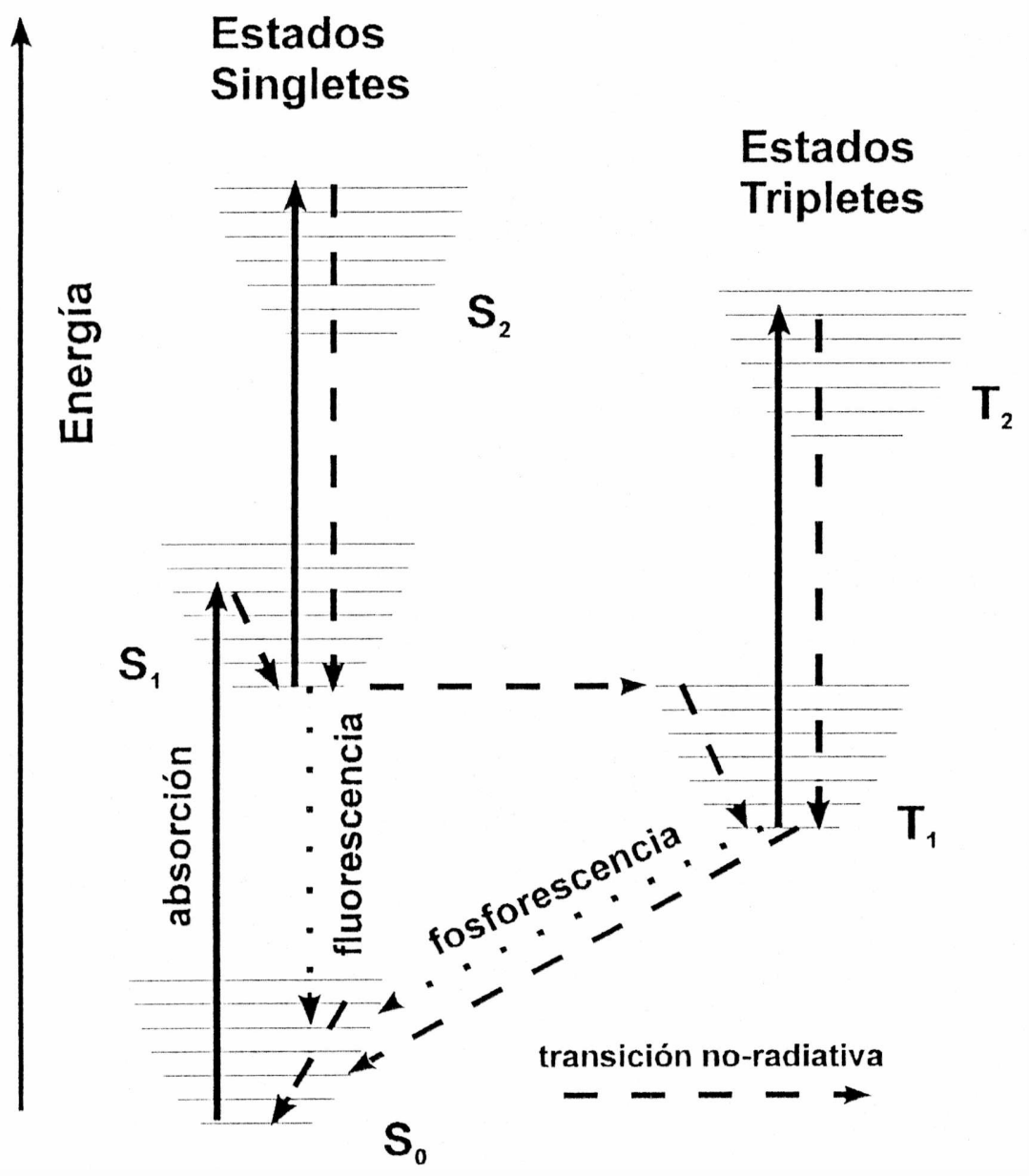

Figura 2.1. Diagrama de Jablonsky para una molécula de colorante. 
Tenemos así estados singletes excitados $S_{1}, S_{2}, S_{3}, \ldots$ y los correspondientes estados tripletes $T_{1}, T_{2}, T_{3}, \ldots$ de menor energía. La distribución esquemática de los niveles de energía en una molécula de colorante, resulta como se muestra en la figura 2.1. Cada uno de los niveles (bandas) vibracionales tiene un ancho definido, dado que estos poseen una estructura fina rotacional que no es mostrada en el diagrama.

Las transiciones entre los estados singletes están permitidas por las reglas de selección espectroscópicas. Ellas dan origen a los intensos espectros de absorción y emisión Las transiciones entre diferentes estados tripletes también están permitidas; pero las transiciones singlete-triplete, conocidas como cruce entre sistemas, están prohibidas. Que la transición esté prohibida significa que esta ocurre con una probabilidad baja respecto de las transiciones permitidas.

La banda de absorción de los colorantes en longitudes de onda larga, se atribuye a la transición desde el estado fundamental electrónico $S_{0}$, al primer estado singlete excitado $S_{1}$. A temperatura ambiente, varias bandas vibracionales del nivel electrónico fundamental se hallan pobladas, entonces serán posibles absorciones con longitudes de onda mayores que a baja temperatura. La desexcitación radiativa $S_{1} \rightarrow S_{0}$ es la responsable de la emisión espontanea conocida como fluorescencia y de la emisión estimulada en láseres de colorante A causa del momento de transición grande de este proceso, la velocidad de la emisión espontanea es bastante alta. Un tiempo de decaimiento típico en colorantes orgánicos es de 5 ns. En general los tiempos de vida de los estados excitados de carácter singlete pueden esperarse en el rango de 1 a 100 ns. Además, asociado con cada estado hay transiciones vibracionales con tiempos de vida del orden de 10 a 0.01 ps.

Una vez que la excitación ocurre a un nivel vibracional de algún estado singlete relativamente alto, digamos $S_{3}$, la molécula inmediatamente comienza a vibrar y en un proceso vibracional en cascada, extremadamente rápido, salta a través de los niveles vibracionales asociados con el estado excitado. Si algún nivel vibracional de $\mathrm{S}_{2}$ es isoenergético o próximo con alguno de $\mathrm{S}_{3}$, entonces existe una alta probabilidad de que en un dado instante la molécula esté vibrando en una forma apropiada para el estado $\mathrm{S}_{2}$. Luego la cascada continúa en $S_{2}$, y si $S_{1}$ cruza a $S_{2}$, el proceso anterior se repite hasta que finalmente la cascada vibracional o proceso de conversión interna lleve la molécula al nivel vibracional más bajo del estado electrónico excitado más bajo, $\mathrm{S}_{1}$. Desde aquí la molécula emite a varios niveles vibracionales del estado base y el espectro de fluorescencia típico resulta como se muestra en la figura 2.2. Este ocurre en una banda ancha y frecuentemente su forma es similar al de absorción. Es su imagen especular si la estructura vibracional es similar en $S_{1}$ y en $S_{0}$. Algunas veces se observa un amplio intervalo en longitudes de onda entre ambos espectros 


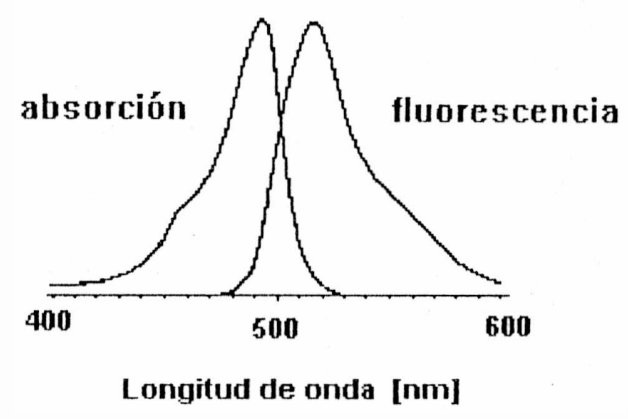

Figura 2.2 : Espectros de absorción y fluorescencia de una molécula típica de colorante, fluoresceina sódica en agua.

\subsection{DESEXCITACIÓN DEL NIVEL $S_{1}$.}

El tiempo de vida de una molécula excitada en el estado $S_{1}$ es del orden de algunos nanosegundos, pero previo a su desexcitacion a niveles más bajos ocurre una relajación rápida desde el estado vibracional excitado hasta el nivel más bajo del estado $S_{1}$. Esta relajación ocurre en tiempos del orden de $100 \mathrm{fs}$ a 1 ps, y es la razón por la cual los espectros de fluorescencia de los colorantes generalmente no dependen de la longitud de onda de excitación. La fluorescencia se produce desde este estado hasta niveles vibracionales de $S_{0}$, con una distribución espectral de la emisión que depende de la probabilidad de cada transición. Dado que las emisiones desde otras bandas de $S_{1}$ resultan casi inexistentes, es evidente que la desexcitación ocurre en esta forma. La relajación que tiene lugar en el estado excitado y la que ocurre luego de la emisión en el estado $\mathrm{S}_{0}$ se atribuyó por mucho tiempo al intercambio energético con moléculas del solvente, que colisionan con la molécula de colorante a una frecuencia del orden de $10^{12}$ colisiones por segundo. Sin embargo, estudios más recientes muestran que aún en fase gaseosa, donde las interacciones ocurren con una frecuencia mucho menor, el espectro de fluorescencia es independiente del nivel vibracional excitado primariamente. Este hecho indica que el proceso de relajación es intramolecular y no necesita de la participación de las moléculas del solvente

Tal como se ha señalado, cuando la molécula de colorante es excitada a un nivel singlete alto, esta desde allí relaja dentro de los picosegundos al nivel vibracional más bajo de $S_{1}$. En este punto hay muchos procesos no radiativos que pueden competir efectivamente con la emisión de luz y entonces reducir la eficiencia de fluorescencia. Se verá luego como por ejemplo estos procesos dependen de una manera compleja de las estructuras moleculares de los colorantes. Los procesos no radiativos pueden agruparse en aquellos que 
causan una relajación directa al estado $S_{0}$ (conversión interna) y aquellos que son responsables del cruce entre sistemas a triplete.

Los estados tripletes y singletes más bajos pueden también ser desactivados por transferencia de energía sin radiación a otras moléculas de colorante. Para que ello suceda la banda de absorción de la molécula aceptora debe superponerse con la banda de fluorescencia o fosforescencia de la primera. La distancia crítica entre moléculas, para la cual la eficiencia cuántica de la emisión se reduce en un factor 2 , puede alcanzar valores de $10 \mathrm{~nm}$ para el caso de transferencia de energía singlete-singlete. Existe así una variedad de procesos por los cuales una molécula excitada retorna al estado fundamental y algunos serán discutidos ahora con más detalle.

\subsubsection{CONVERSIÓN INTERNA}

El decaimiento no radiativo desde el estado singlete excitado más bajo $S_{1}$, al estado fundamental $\mathrm{S}_{0}$ es el mayor responsable por la pérdida de eficiencia de fluorescencia en los colorantes orgánicos. Dependiendo de la estructura molecular del colorante y de las propiedades del solvente, la velocidad de esta relajación puede variar en muchos ordenes de magnitud. Dado que existen diferentes características estructurales que contribuyen al decaimiento no radiativo $S_{1} \rightarrow S_{0}$, la relación entre estructura molecular y eficiencia de fluorescencia puede resultar enigmática.

Una de estas características es la movilidad estructural. Las estructuras moleculares planas y rigidas favorecen las altas eficiencias de fluorescencia. Como ejemplos contrapuestos pueden mencionarse la fenolftaleína y la fluoresceína, con eficiencias que van desde ser prácticamente nula a alcanzar un valor del $90 \%$ respectivamente. Que los colorantes con estructuras completamente rigidas tendrían una eficiencia de fluorescencia muy alta, puede verse también a partir del comportamiento de las eficiencias de estos compuestos con la temperatura. La eficiencia de fluorescencia de la rodamina $\mathrm{B}$ en etanol depende de la temperatura como sigue. A $25^{\circ} \mathrm{C}$ es aproximadamente del $40 \%$. Disminuyendo la temperatura llega a ser cercana al 100\%, mientras que cae a un porcentaje pequeño en etanol en ebullición. Este efecto puede ser atribuido a la movilidad de los grupos dietilamino, la cual aumenta con la temperatura. Si estos grupos son inmovilizados, como en el caso de la rodamina 101, la eficiencia de fluorescencia llega a ser virtualmente del $100 \%$, independientemente de la temperatura. La movilidad estructural aumentaría la conversión interna, pero dado que este último proceso no depende sólo de dicha movilidad, puede ocurrir que colorantes con una estructura rígida tengan baja eficiencia de fluorescencia, o también que teniendo grupos móviles sean altamente fluorescentes. La 
razón de la alta eficiencia de fluorescencia de colorantes como la rodamina $110(85 \%$ en solución alcohólica, independientemente de la temperatura), puede entenderse en términos de la densidad de electrones $\pi$ en el enlace $C=N$ cuando la molécula está excitada. Si esta densidad es alta, la energía térmica de las moléculas del solvente no será suficiente para torcer los grupos amino fuera del plano molecular. La viscosidad de los solventes puede jugar un rol importante en la movilidad estructural de las moléculas de colorante, permitiendo o no apartamientos de la forma plana de las mismas.

Aún cuando el cromóforo de algunos colorantes sea completamente plano y rígido, otro mecanismo de conversión interna puede estar actuando. Este involucra la conversión del nivel vibracional más bajo del estado excitado $S_{1}$ a un nivel vibracional alto del estado fundamental $S_{0}$, el cual entonces relaja rápidamente al nivel vibracional más bajo de $S_{0}$. Este proceso es en principio independiente de la temperatura y viscosidad del solvente. El mismo se relaciona con las vibraciones de los átomos de hidrógeno pegados directamente al cromóforo del colorante. El reemplazo del hidrógeno por deuterio debería reducir la efectividad de este mecanismo, habida cuenta de la reducción de energía por las vibraciones de los átomos más pesados, y en consecuencia observarse un aumento en la eficiencia de fluorescencia. Esto se observa, por ejemplo, en rodamina 110 y cresil violeta. Este mecanismo debería también ser más efectivo al disminuir la diferencia de energía entre $S_{1}$ y $\mathrm{S}_{0}$

Sumados a los mecanismos de conversión interna, hay varios procesos intramoleculares que pueden causar la extinción de la fuorescencia. Por ejemplo, puede ocurrir una transferencia de carga reversible entre un grupo de la molécula donante y el cromóforo excitado, haciendo que se pierda la excitación electrónica. El hecho de que una gran variedad de procesos de relajación no radiativos pueda competir con la emisión de la fluorescencia, hace que resulte muy dificil dilucidar la relación entre estructura molecular y eficiencia cuántica de fluorescencia de los colorantes orgánicos.

\subsubsection{CRUCE ENTRE SISTEMAS $\left(\mathrm{S}_{1} \rightarrow \mathrm{T}_{1}\right)$.}

Además del decaimiento no radiativo directo al estado fundamental, una molécula excitada en el estado $S_{1}$ puede entrar en el sistema de estados tripletes y relajar al nivel más bajo de estos, $T_{1}$. Este cruce entre sistemas, en colorantes típicos, ocurre en tiempos del orden de los $50 \mathrm{~ns}$, mientras que el tiempo de vida del estado triplete puede ser del orden de los milisegundos.

La transición radiativa $\mathrm{T}_{1} \rightarrow \mathrm{S}_{0}$, se conoce como fosforescencia. Como es de esperar para las transiciones de spin prohibidas, el tiempo de vida de esta es extremadamente largo, 
yendo desde los milisegundos a varios segundos para moléculas con un acoplamiento spinorbita pequeño. Los procesos lentos de extinción pueden conducir a una desactivación no radiativa en solución líquida, determinando que la fosforescencia sea apreciable sólo a bajas temperaturas, por ejemplo $77 \mathrm{~K}$ en soluciones sólidas

Ciertas características estructurales de los colorantes orgánicos influencian la velocidad del cruce entre los sistemas. El cruce desde el singlete al triplete, siendo un proceso prohibido, es comparativamente lento considerando la pequeña diferencia de energía entre $S_{1}$ y $T_{1}$. Sin embargo, su velocidad varía de una forma peculiar con la estructura molecular del colorante. Una regla establece que en un colorante donde los electrones $\pi$ del cromóforo puedan hacer un rizo cuando oscilan entre los grupos terminales, la eficiencia a tripletes será más alta que en un compuesto relacionado donde este rizo esté bloqueado. Podría decirse que los electrones circulando crean un momento magnético orbital, el cual se acopla con el spin de los electrones. Esto incrementa el acoplamiento spin-orbita y entonces mejora la velocidad del cruce entre sistemas, aumentando la eficiencia del pasaje a tripletes. El acuerdo hallado con las observaciones experimentales da muestras de la utilidad de esta regla, que predice cualitativamente las eficiencias de este pasaje para muchos colorantes orgánicos. La regla predice eficiencias relativamente altas en colorantes con cromóforo en forma de anillo, como por ejemplo clorofilas y ftalocianinas. Por otra parte, las oxazinas y aquellos compuestos similares en los que el oxígeno es reemplazado por un átomo de carbono tetraédrico, al no estar permitido el paso de los electrones $\pi$ sobre estos, la predicción es una muy baja eficiencia de formación de tripletes.

El cruce entre sistemas, si bien depende esencialmente de la distribución de electrones $\pi$ en el cromóforo, puede mejorarse si el colorante es sustituido con elementos más pesados, lo cual incrementa el acoplamiento spin-orbita. El mismo efecto ocurre (el aumento en la eficiencia del pasaje a tripletes) si el compuesto orgánico es disuelto en un solvente conteniendo átomos pesados como sustituyentes. Esto se debe a que la transición sin radiación desde un estado singlete excitado a un estado triplete puede ser inducida no sólo por perturbaciones internas (acoplamiento spin-orbita) sino también por perturbaciones externas, como pueden ser colisiones con moléculas de oxígeno en solución.

\subsection{ABSORCIÓN DE LUZ EN LOS ESTADOS $\mathrm{S}_{1} \mathrm{Y} \mathrm{T}_{1}$}

Al igual que con las moléculas en el estado $S_{0}$, hay espectros de absorción bien definidos asociados con las moléculas que están en los estados excitados $S_{1}$ y $T_{1}$. Estos espectros son muy dificiles de medir. La determinación del espectro de absorción del estado 
excitado $S_{1}$, requiere normalmente el empleo de técnicas láser debido al corto tiempo de vida de este estado. El espectro de absorción de moléculas en el estado triplete más bajo $T_{1}$, es más fácil de obtener si el compuesto está embebido en una matriz sólida, donde el tiempo de vida del triplete es muy largo (en algunos casos varios segundos). Si la solución sólida es excitada con una fuente de luz muy intensa, se logra una población apreciable del estado triplete y el espectro puede ser determinado sin demasiada dificultad, en muchos casos simplemente con un espectrofotómetro comercial.

Las transiciones triplete-triplete están permitidas y la absorción óptica asociada con estas es fuerte. La banda de absorción en la transición $T_{1} \rightarrow T_{2}$ generalmente se superpone con el espectro de emisión $S_{1} \rightarrow S_{0}$, ocurriendo en muchos casos lo propio con el espectro de absorción $S_{1} \rightarrow S_{n}$. Ello debilita la intensidad de la fluorescencia al punto de llegar a anularla.

\subsection{EFECTOS DEL MEDIO SOBRE EL COMPORTAMIENTO DE LOS COLORANTES.}

Los procesos no radiativos en moléculas de colorante no sólo se relacionan con la estructura molecular de estos, sino también con el medio en el que estos compuestos están inmersos. Así por ejemplo, se sabe que la fluorescencia de los colorantes es extinguida por ciertos aniones. La eficiencia de extinción depende fuertemente de la naturaleza química del anion, resultando muy importante en el caso del iodo. El estado excitado del colorante podría ser desactivado por un mecanismo de transferencia de carga. El cromóforo de muchos colorante orgánicos porta una carga positiva como resultado de que estos colorantes son sales iónicas con un anion, comúnmente cloro o iodo. Que la fluorescencia sea afectada por este anion dependerá de la concentración y polaridad del solvente. Aparentemente las sales del colorante están totalmente disociadas en un solvente polar tal como etanol y no tienen tiempo de alcanzar a las moléculas excitadas durante su tiempo de vida. En otros solventes (cloroformo) la sal no se disocia y pude tomar parte en un mecanismo de interacción con la especie excitada. Existen otros compuestos además de los aniones que pueden extinguir la fluorescencia por un mecanismo similar.

Otro proceso por el cual los estados excitados, tanto de singletes como tripletes, son desactivados externamente puede operar si la molécula de intercambio tiene un nivel de energía igual o más bajo que aquel del estado a ser desexcitado. Bajo condiciones favorables la transferencia de energía puede ocurrir sobre distancias de alrededor de $10 \mathrm{~nm}$. En solución líquida, donde los reactivos pueden aproximarse uno a otro muy cerca, los procesos de transferencia de energía son muy eficientes, siempre que el tiempo de difusión 
sea más corto que el tiempo de vida del estado excitado. A altas concentraciones, la fluorescencia de la mayoría de los compuestos orgánicos es extinguida por la fuerte interacción entre las moléculas excitadas y aquellas de la misma especie en el estado fundamental. En el caso de la piridina y de algunos otros compuestos, se ha observado la aparición de una nueva banda de fluorescencia cuando se aumenta la concentración de la solución. Esta nueva banda se debe a los dímeros que existen sólo en el estado excitado (excímeros). Luego de la emisión de un fotón, ellos se disocian inmediatamente en monómeros en el estado fundamental. Similarmente, una molécula excitada puede reaccionar con una molécula de un compuesto diferente, de solvente o de otro soluto, para formar un complejo excitado, el cual se disociará en sus componentes después de la desexcitación radiativa.

\subsubsection{AGREGACIÓN DE LAS MOLÉCULAS DE COLORANTE.}

Los colorantes orgánicos en solución acuosa tienen la tendencia a formar dímeros y agregados más altos, con espectros de absorción distintivos de cada especie. Los dímeros normalmente tienen una banda de absorción fuerte en longitudes de onda más corta que los monómeros y con frecuencia una banda adicional más débil del lado de las longitudes de onda larga. Ellos son en general poco o nada fluorescentes. El equilibrio entre monómeros y dímeros se corre hacia estos últimos cuando se aumenta la concentración del colorante y con la disminución de la temperatura.

Diferentes factores han sido sugeridos como los responsables de la agregación de los colorantes orgánicos. Se ha asumido que una fuerza de dispersión atractiva entre cromóferos altamente polarizables juega un rol importante; en tanto que la constante dieléctrica alta del agua reduce la repulsión culombiana entre moléculas cargadas. Mientras la agregación en agua es común con muchos colorantes, ésta usualmente no ocurre en solventes orgánicos, aún en concentraciones muy altas. Es posible eliminar la agregación de colorantes en solución acuosa con el agregado de compuestos orgánicos. Presumiblemente estos compuestos formen una celda alrededor del colorante hidrofóbico y entonces resguarden a este de las otras moléculas de colorante y de las de agua. En acuerdo con la formación de este cluster, se ha observado que el tiempo de relajación rotacional de las moléculas de colorante es más largo en estas soluciones [Drexhage] 


\subsubsection{EFECTOS DEL SOLVENTE SOBRE LAS TRANSICIONES ELECTRÓNICAS.}

Cuando se considera el cambio en la longitud de onda de absorción de un determinado compuesto al pasar de fase gaseosa a una solución en fase condensada, uno debe considerar el cambio en la energía de excitación de ese compuesto. Este puede ser atribuido a la constante dieléctrica del solvente y a efectos de solvatación específicos, tales como enlaces hidrógeno. Las anilinas muestran ambos efectos claramente [Wiberg]

Los estados excitados dipolares deberían ser estabilizados por un aumento de la constante dieléctrica más que los estados base no polares. En estos casos, con un incremento en la constante dieléctrica de los solventes, la energía de la emisión debería reducirse, originando un corrimiento al rojo. Esto se observa yendo de fase gaseosa a un solvente y yendo también de un solvente no polar a otro relativamente polar, como puede ser acetonitrilo o dioxano. En sustancias para las cuales el estado fundamental es más polar que el estado excitado, se puede observar el efecto opuesto.

Cuando se considera el efecto de un solvente tal como etanol o agua debemos tener presente el principio de Franck-Condon. Durante una transición electrónica ni la posición de los núcleos en el compuesto, ni la posición de las moléculas de solvente en torno a éste, cambian. En etanol, la anilina estará ligada por puente hidrógeno con los protones del solvente, y esto hace que baje la energía de su estado fundamental cuando se la compara con la de la molécula en fase vapor. Por otra parte, en el estado excitado el enlace hidrógeno es menos importante a causa de la carga positiva del nitrógeno. Si las moléculas de solvente pudieran moverse de manera de alcanzar una posición más favorable para la interacción dipolo-dipolo con la molécula excitada, se lograría alguna estabilización. Pero ello no es posible en el tiempo de la transición electrónica. Disolviendo anilina en etanol se baja entonces la energía de su estado fundamental más que la del estado excitado, con lo cual aumenta la energía de excitación, produciéndose un corrimiento al azul de su espectro de absorción. En agua el corrimiento al rojo causado por el efecto de la constante dieléctrica, es cancelado por el corrimiento al azul debido al cambio en los enlaces por puente hidrógeno. Esto hace que la anilina tenga esencialmente el mismo máximo de absorción en agua que en fase gaseosa. Cuando el estado excitado es más polar que el estado base, la energía de excitación decrece yendo de la fase gaseosa a un solvente, y yendo de un solvente a otro más polar. Este es el caso del nitrobenceno para el cual los enlaces por puente hidrógeno se dan entre los oxígenos del grupo nitro y los protones del solvente. En el estado excitado los enlaces hidrógeno deberian ser más importantes, dado que las cargas negativas sobre los oxigenos aumentan respecto de aquellas en el estado fundamental de la molécula. Entonces el enlace hidrógeno debería disminuir la energía del estado excitado más que la del estado base, originando un corrimiento al rojo. 


\subsection{CÁlCUlo DEL TIEMPO DE VIDA RADIATIVO DEL PRIMER SINGLETE EXCITADO $\left(S_{1}\right)$.}

Como hemos visto, existen diferentes procesos por los cuales una molécula excitada puede regresar directa o indirectamente a su estado fundamental. Uno de estos procesos es la transición radiativa desde el primer estado singlete excitado, $\mathrm{S}_{1}$, al estado fundamental de la molécula, $S_{0}$. Si esta emisión (fluorescencia) ocurre espontáneamente, el tiempo de vida radiativo, $\tau_{\mathbf{r}}$, se relaciona con el coeficiente de Einstein para la emisión espontánea, $\mathbf{A}, \mathrm{y}$ con la intensidad de oscilador, $f$, de la banda de absorción pertinente, mediante la siguiente expresión:

$$
\mathrm{A}=\tau_{\mathrm{r}}^{-1}=\frac{8 \pi^{2} \mathrm{n}^{2} \mathrm{e}^{2}}{\mathrm{~m} . \mathrm{c}} \cdot v^{2} \cdot f
$$

donde $\mathbf{n}$ es el índice de refracción del medio, e y $\mathbf{m}$ la carga y masa del electrón respectivamente, c la velocidad de la luz y $v$ el número de onda correspondiente al centro de la banda de absorción. Esta relación es válida si el ancho medio de la banda de emisión es pequeño y su posición no está corrida significativamente respecto de aquella correspondiente a la banda de absorción. Dado que los valores de $f$ de la transición son cercanos a la unidad en muchos colorantes, el tiempo de vida radiativo, $\tau_{\mathbf{r}}$, es típicamente del orden de unos pocos nanosegundos.

Ocurre que los espectros de fluorescencia son generalmente anchos y muestran un corrimiento Stokes considerable. En estos casos el tiempo de vida radiativo puede ser calculado con la ayuda de la relación de Strickler y Berg

$$
\tau_{\mathbf{r}}^{-1}=\mathbf{a} \cdot \mathbf{n}^{2} \cdot \frac{\mathbf{g}_{1}}{\mathbf{g}_{u}} \cdot \frac{\int \mathbf{I}(v) \mathbf{d} v}{\int v^{-3} \mathbf{I}(v) \mathbf{d} v} \cdot \int \frac{\varepsilon(v) \mathbf{d} v}{v}
$$

donde $\mathbf{I}(v)$ es la intensidad del espectro de fluorescencia, que debería ser medida en términos del número relativo de cuantos en cada frecuencia más que en unidades de energía. a es un factor constante. $\mathbf{g}_{\mathbf{l}}$ y $\mathbf{g}_{\mathbf{u}}$ representan la degeneración de los estados electrónicos superior e inferior $\varepsilon(v)$ es el coeficiente de extinción molar y la integral se extiende sobre toda la banda de absorción.

Si la banda de emisión es estrecha y la absorción y emisión ocurren en la misma longitud de onda, $v$ puede ser considerada como una constante. Luego el factor $v^{-3}$ puede sacarse fuera de la integral, con lo cual la Eq. (2) se reduce a la ecuación (1) como es de esperar para el caso de transiciones atómicas 
Esta ecuación relaciona el tiempo de vida radiativo de las moléculas excitadas con sus coeficientes de absorción $(\varepsilon(v))$. Luego $\tau_{r}$ puede ser calculado a partir del conocimiento de los espectros de absorción y de fluorescencia de estas moléculas.

Cabe señalarse que una ecuación análoga para semiconductores, la cual relaciona el tiempo de vida radiativo de un par electrón-hueco en un semiconductor con el espectro de absorción de éste, fue deducida por Roosbroeck y Shockley. La misma se indica a continuación.

$$
\tau_{r}^{-1}=\frac{n_{0}+p_{0}}{n_{0} \cdot p_{0}} \cdot \frac{8 \pi}{c^{2}} \cdot \int \frac{\alpha \cdot n^{2} v^{2}}{\exp \left(\frac{h v}{k T}\right)-1} \cdot d v
$$

donde $\mathbf{n}_{0}$ y $\mathbf{p}_{0}$ son las concentraciones en equilibrio de electrones en la banda de conducción y de huecos en la banda de valencia respectivamente, $\alpha$ es el coeficiente de absorción, y la integral se toma sobre toda la banda de absorción.

Puesto que estas dos ecuaciones ( 2 y 3 ) se refieren a sistemas distintos y tienen formas bastante diferentes, no resulta obvio ver que están relacionadas. Las diferencias entre estas dos ecuaciones aparecen por el uso de distintas nomenclaturas, a causa de la bimolecularidad del proceso de recombinación en seniconductores, que requiere la participación de un electrón y un hueco en cada absorción o emisión entre bandas. Pero principalmente a partir del corrimiento del espectro de fluorescencia molecular, hacia frecuencias por debajo del extremo de la banda de absorción, donde en general el coeficiente de absorción no se conoce. Bolton y Archer demuestran que ambas ecuaciones pueden ser deducidas a partir de un tratamiento teórico común, el cual se basa en la suposición de bandas termalizadas, el uso del principio de reversibilidad microscópica, y para el caso de cromóferos moleculares, la validez de la relación de la imagen especular. Esta última se debe a que su tratamiento no se apoya sobre suposiciones concernientes a los momentos de transición. Es precisamente este hecho el que diferencia el camino de deducción seguido por Strickler y Berg de su ecuación, que no requiere el uso de la relación de simetría especular para las propiedades de absorción y emisión en moléculas de colorante 


\subsection{FORMAS DE EXCITACIÓN ELECTRÓNICA.}

Hasta aquí hemos considerado la excitación de un electrón desde un orbital molecular $\pi$ a otro, comúnmente referida como una transición $\pi \rightarrow \pi^{*}$. Vimos que hay dos clases de transiciones, aquellas que involucran un estado singlete excitado y aquellas que involucran a un estado triplete excitado. Sólo la primera da una banda de intensidad razonable; la excitación a un estado triplete formalmente está prohibida y da una banda muy débil. Esto no significa que el estado triplete no sea importante para muchos singletes excitados que decaen a un estado triplete de menor energía. Este último tiene un tiempo de vida largo porque la transición al estado fundamental es poco probable. Entonces muchas reacciones fotoquímicas involucrarán a este estado triplete.

Otra transición electrónica importante es aquella que involucra la excitación de un electrón de un par desapareado (nitrógeno $\mathrm{u}$ oxígeno) a un enlace molecular $\pi$ antienlazante. Este tipo de transición es una característica común de los espectros de las aminas no saturadas, tal como piridina, y de las cetonas y aldehidos; y se refiere a una transición $n \rightarrow \pi^{*}$.

Resulta importante distinguir entre estos dos tipos de transiciones. Por ejemplo, antes que se conocieran las transiciones $\mathbf{n} \rightarrow \pi^{*}$, aquellas de baja energía de las azinas se atribuían a transiciones $\pi \rightarrow \pi^{*}$, y esto condujo a valores erróneos en la deslocalización de la energía, basada en el tratamiento de los enlaces de valencia. A veces resulta dificil lograr esta distinción. Las herramientas principales para ello lo constituyen los efectos del solvente, la polarización de las bandas y la intensidad de las mismas. Es fácil ver que las transiciones $\mathrm{n} \rightarrow \pi^{*}$ son de baja intensidad. Podemos considerar al par desapareado teniendo un orbital híbrido $\mathrm{sp}^{2}$. La componente $\mathrm{s}$ es una función par y la integral de ésta con dos funciones impares, la coordenada y la función de onda $\pi$ en la dirección perpendicular al enlace $\sigma$, será distinta de cero Sin embargo, esta será pequeña porque la distancia desde el centro del orbital $2 \mathrm{~s}$ al centro de uno de lóbulos $\pi$ es pequeña. La componente $\mathrm{p}$ del par desapareado será cero en la integral correspondiente, dado que es antisimétrica con respecto a la dirección de su eje mayor. Entonces la longitud del momento de transición total será un tercio de la integral (la contribución de la parte s del orbital), alrededor de $0.2 \AA$. Puede verse que la transición es permitida sólo en la dirección perpendicular al sistema de referencia $\sigma$ de la molécula, y entonces la transición $n \rightarrow \pi^{*}$ es permitida en una dirección diferente a las transiciones $\pi \rightarrow \pi^{*}$

Los estados excitados que resultan de una transición $n \rightarrow \pi^{*}$ tienen un momento dipolar considerable, dado que un electrón es tomado normalmente de un $\mathrm{O}$ o $\mathrm{N}$ y ubicado en un orbital $\pi$. Como resultado de colocar una carga positiva sobre el heteroátomo, la banda de absorción de tales transiciones siempre muestran un fuerte corrimiento al azul 
yendo de solventes sin enlace hidrógeno a alcoholes o agua. Este corrimiento es uno de los criterios empleados para discernir el tipo de transición.

\subsection{PROPIEDADES FÍSICAS DE LAS CIANINAS.}

Como ya fuera señalado en el capítulo anterior, las cianinas constituyen una familia de colorantes polimetínicos ampliamente utilizados en diversas áreas tecnológicas e industriales, principalmente en el desarrollo de láseres [Arthurs, 1972; Mialocq, 1979] y en la industria fotográfica. El mayor interés en fotobiología está dado por la similitud estructural de las cianinas con una variedad de compuestos naturales [Sheves], y por su uso como sondas fluorescentes en el estudio de las propiedades de biomembranas [Grieser]. En la figura 2.3 se representa la estructura del isómero todo-trans de las cianinas que fueron motivo de estudio en esta tesis.
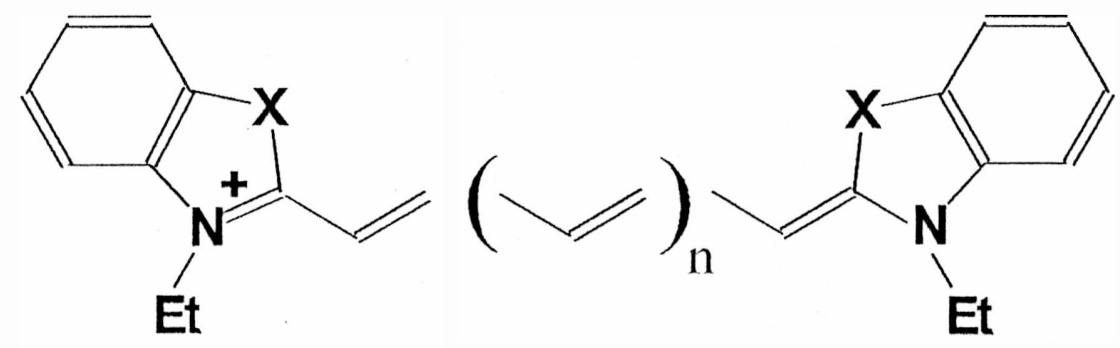

\begin{tabular}{lll}
\multicolumn{1}{c}{} & & \\
CIANINA & $\mathrm{n}$ & $\mathrm{X}$ \\
& & \\
Dietiloxacarbocianina (DOCI) & 0 & $\mathrm{O}$ \\
Dietiloxadicarbocianina (DODCI) & 1 & $\mathrm{O}$ \\
Dietiltiacarbocianina (DTCI) & 0 & $\mathrm{~S}$ \\
Dietiltiadicarbocianina (DTDCI) & 1 & $\mathrm{~S}$
\end{tabular}

Figura 2.3. 
Las cianinas presentan gran solubilidad en alcoholes, alta absorción de radiación en el visible ( $\varepsilon \approx 10^{5} \mathrm{M}^{-1} \mathrm{~cm}^{-1}$ ) y en general son intensamente fluorescentes. Además de estas propiedades, que son comunes a la mayoría de los colorantes, las cianinas se caracterizan por presentar isomerización trans-cis fotoinducida (fotoisomerización) [Dempster]. La absorción de luz visible desde el estado fundamental de la forma térmicamente estable $(\mathrm{N})$, conduce a la misma al primer estado singlete excitado $\left(S_{1}^{N}\right)$, mediante una transición $\pi \rightarrow \pi^{*}$. Desde este estado la cadena polimetínica puede rotar alrededor de alguna de las uniones carbono-carbono que la componen, dando lugar a la formación de un fotoisómero (P)

Dado que la eficiencia cuántica de fluorescencia de las cianinas disminuye al aumentar la temperatura, y la velocidad de emisión espontanea es independiente de este parámetro, existe por lo menos un proceso activado que compite con la fluorescencia. Uno de estos procesos es la fotoisomerización del colorante.

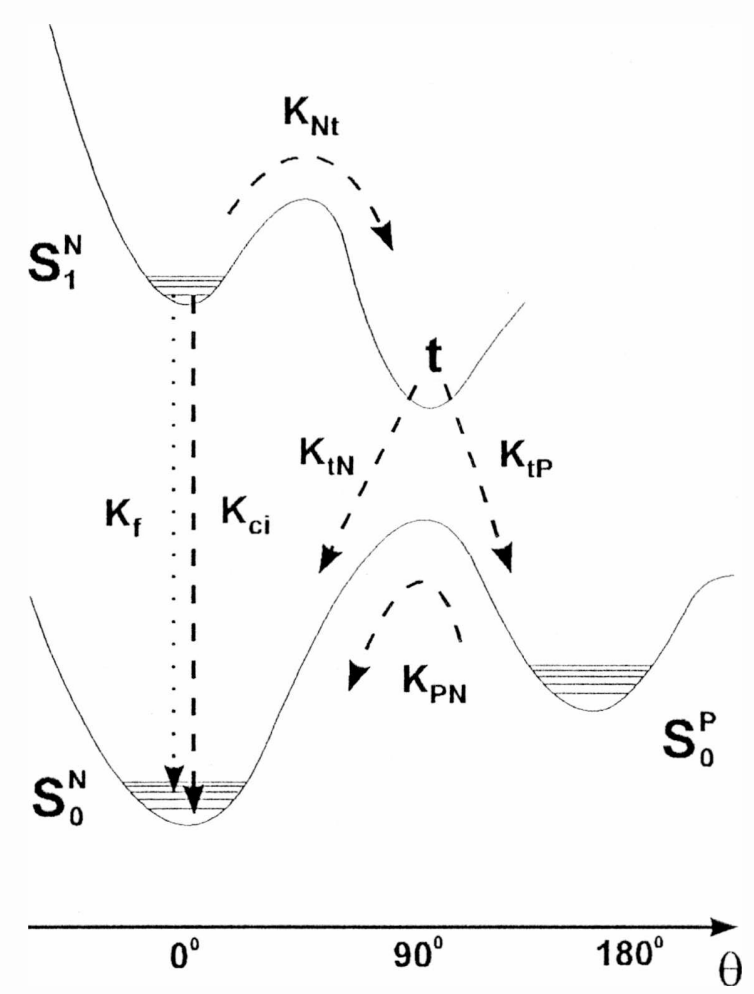

Figura 2.4. Representación unidimensional de las superficies de energia potencial, en términos de la coordenada de rotación $0 . K_{\text {Ig }}$ son las velocidades de desactivación corespondicntes a los distintos procesos señalados. 
De acuerdo con diferentes modelos propuestos para describir la fotoisomerización de las cianinas, este proceso, el pasaje desde el estado excitado de la especie normal al fundamental del fotoisómero, tiene una instancia intermedia llamada estado "twisted". A diferencia de las formas normal y fotoisomérica, cuyas estructuras moleculares serían planas, en este estado "torsionado" los grupos extremos de la molécula se hallan a $90^{\circ}$ uno respecto del otro, por rotación alrededor de alguna unión de la cadena polimetínica [Dietz]. Este estado no espectroscópico, que se indica con la letra $\mathbf{t}$ en la figura 2.4 , decae muy rápidamente por medio de una transición no radiativa hacia la superficie de energía potencial del estado fundamental, pudiendo seguir luego dos caminos distintos. Uno de ellos consiste en completar la rotación de los grupos extremos $\left(\theta=180^{\circ}\right)$, dando origen a la especie transiente (fotoisómero). El otro camino consiste en desandar la rotación primera, regresando la molécula a su forma normal. Estos dos procesos no son activados y compiten entre sí con distinta probabilidad.

En acuerdo con el modelo descripto, la especie normal excitada puede alcanzar su estado fundamental mediante diferentes procesos. Uno de ellos es la conversión interna no activada, que compite con la fluorescencia y con el pasaje hacia el estado twisted (t). Otro es la vuelta pasando por el estado $\mathbf{t}$, sin que se forme el fotoisómero. El último de los caminos posibles es la retroisomerización, esto es, la conversión del fotoisómero en la especie normal. Esta isomerización tiene lugar sobre la superficie potencial del estado fundamental y ocurre en una escala de tiempos mucho mayor que la de los demás procesos arriba indicados (milisegundos frente a nanosegundos). 


\section{CAPÍTULO 3: POLARIZACIÓN DE LA FLUORESCENCIA.}

\subsection{INTRODUCCIÓN}

Cuando un conjunto de moléculas distribuidas al azar es excitado con un haz de luz, se crea una distribución orientacional no uniforme de los momentos de transición de aquellas moléculas que fueron excitadas. Esta anisotropia puede decaer en el tiempo por difusión rotacional de las moléculas y entonces conducir a la despolarización de la radiación emitida y al mismo tiempo a un decaimiento del dicroismo inducido inicialmente. El estudio del movimiento rotacional de moléculas en líquidos por despolarización de la fluorescencia, está limitado por la respuesta temporal de la fuente de excitación o del sistema de detección. Utilizando técnicas láser de picosegundos es posible seguir la dinámica rotacional en la región de tiempo de los subnanosegundos

Perrin (1926) fue el primero en formular la relación existente entre la despolarización de la fluorescencia y el movimiento rotacional Browniano para una molécula esférica. Usando la teoría de Perrin, junto con una extensión de la teoría debida a Weber (1952), se han realizado en el pasado numerosos experimentos para determinar tiempos medios de relajación rotacional. Estos se relacionan por las leyes hidrodinámicas con el volumen y con la forma de las moléculas, permitiendo obtener información sobre la estructura y conformación de las mismas

Para una molécula cuya difusión rotacional alrededor de cada uno de sus ejes principales resulte considerablemente diferente, la descripción isotrópica debe ser reemplazada por un modelo anisotrópico de la rotación. Perrin (1934) y Memming estudiaron el movimiento rotacional de moléculas simétricas, y posteriormente Weber (1971) y Tao, este último usando el formalismo de las funciones de distribución, obtuvieron la formulación para la difusión de un rotor asimétrico. La expresión más general para la despolarización de la fluorescencia dependiente del tiempo, causada por difusión rotacional anisotrópica, que contiene como casos particulares a las anteriores, es aquella debida Chuang y Eisenthal. Existen diferencias entre las distintas expresiones, que surgen a partir de un tratamiento no general del problema o por no usar las expresiones correctas para las probabilidades de transición [Tao]. Weber y Chuang coinciden en la expresión para oblados. Esta la dedujo primero Memming, que también obtuvo la expresión de los prolados. Tao y Weber tienen la misma expresión para los oblados sólo si la anisotropía en ausencia de movimiento Browniano alcanza su valor teórico máximo $(0.4)$

La despolarización de la fluorescencia estudiada en estado estacionario presenta algunos inconvenientes. Se podría citar por ejemplo, que el tiempo de decaimiento natural de la fluorescencia debe ser conocido, y frecuentemente no están determinados con precisión. 
Es necesario alterar la viscosidad y/o la temperatura del medio, sin saber en algunos casos los efectos que este cambio produce sobre la conformación de la molécula. Los coeficientes de difusión en general no pueden determinarse individualmente (sólo la media puede ser determinada). Para evitar algunas de estas dificultades, lo mejor es seguir el proceso de relajación rotacional directamente midiendo el decaimiento de la fluorescencia polarizada como función del tiempo. No obstante, su estudio en estado estacionario permite en ciertos casos obtener información de interés a un propósito dado. Particularmente ello es así cuando se tiene un único coeficiente de difusión dominante en el proceso de decaimiento.

\subsection{TEORÍA DE LA DESPOLARIZACIÓN DE LA FLUORESCENCIA.}

Comenzaré analizando una molécula fluorescente cuyo dipolo de transición es el mismo para el proceso de absorción y de emisión. Sean $(\theta, \phi)$ los ángulos que definen la orientación de $\mu$ respecto de un sistema de coordenadas fijos en el espacio $X, Y, Z$ (ver figura 3. 1). Asumamos que la luz de excitación viaja en la dirección $X$, con polarización lineal en $Z$. La muestra fluorescente está en el origen de coordenadas y su fluorescencia es observada en la dirección $\mathrm{Y}$. Nuestro interés está en determinar la componente de la fluorescencia polarizada en la misma dirección que la excitación $\left(\mathrm{I}_{z}\right)$, y aquella en la dirección perpendicular a esta última $\left(\mathrm{I}_{\mathrm{X}}\right)$.

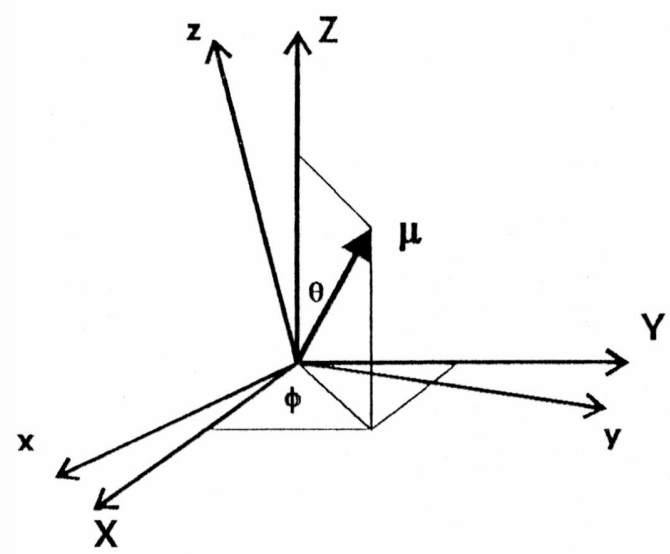

Figura 3.1. X, Y,Z es el sistema de referencia usado para describir la geometria experimental. $\mathrm{x}, \mathrm{y}, \mathrm{z}$ es un sistema de ejes fijo a la molécula fluorescencte. 
La anisotropía de la fluorescencia se define como:

$$
\mathbf{r}=\frac{\mathbf{I}_{\mathbf{z}}-\mathbf{I}_{\mathbf{x}}}{\mathbf{I}_{\mathrm{z}}+2 \mathbf{I}_{\mathbf{x}}}
$$

Ahora bien, sea $f(\theta, \phi, t)$ la probabilidad de que el vector $\mu$ esté orientado en $(\theta, \phi)$ en el instante $\mathrm{t}$, y $\mathrm{G}\left(\theta_{0}, \phi_{0} ; \theta, \phi, \mathrm{t}\right)$ la función de evolución de aquella probabilidad, esto es, la probabilidad de que si $\mu$ está orientado en $\left(\theta_{0}, \phi_{0}\right)$ a $t=0$, entonces estará orientado en $(\theta, \phi)$ al tiempo t. A partir de estas definiciones, es claro que

$$
f(\theta, \phi, t)=\int_{0}^{2 \pi} d \phi_{0} \int_{0}^{\pi} \operatorname{sen} \theta_{0} d \theta_{0} . f\left(\theta_{0}, \phi_{0}, t_{0}\right) \cdot G\left(\theta_{0}, \phi_{0}, \theta, \phi, t\right)
$$

y $\mathrm{f}\left(\theta_{0,} \phi_{0}\right)$ es la distribución inicial de orientaciones de los momentos de transición.

La ecuación anterior también define a $\mathrm{G}$ como la función de Green de la ecuación diferencial que satisface $f(\theta, \phi, t)$.

La probabilidad de que una molécula con dipolo de transición $\mu$ absorba un fotón cuyo vector eléctrico está polarizado en la dirección $Z$, es proporcional a $|\boldsymbol{\mu} \cdot \mathbf{z}|^{2}=\mu_{\mathrm{z}}^{2}$. Luego la distribución inicial normalizada es:

$$
f\left(\theta_{0}, \phi_{0}\right)=\frac{3}{4 \pi} \cdot \cos ^{2} \theta_{0}
$$

Expandiendo la función de Green $\mathrm{G}\left(\theta_{0}, \phi_{0} ; \theta, \phi, t\right)$ en harmónicos esféricos resulta :

$$
\mathbf{f}(\theta, \phi, t)=\frac{1}{4 \pi}\left[1+2\left\langle\mathbf{P}_{2}(\mu(0) \cdot \mu(t))\right\rangle \mathbf{P}_{2}(\cos \theta)\right]
$$

donde $\mathrm{P}_{2}$ es el polinomio de Legendre de orden 2 , y la función de correlación,

$\left\langle P_{2}(\mu(0) \cdot \mu(t))\right\rangle=\frac{1}{4 \pi} \cdot \iint \operatorname{sen} \theta d \theta d \phi \iint \operatorname{sen} \theta_{0} d \theta_{0} d \phi_{0} P_{2}(\mu(0) \cdot \mu(t)) \cdot f\left(\theta_{0}, \phi_{0}\right) \cdot G\left(\theta_{0}, \phi_{0} ; \theta, \phi, t\right)$

Cuando la emisión del dipolo oscilante $\mu$, orientado en $(\theta, \phi)$, es observada en la dirección $\mathrm{Y}$, la componente de esta emisión en $Z$ (componente paralela a la polarización de 
excitación) y la componente en $\mathrm{X}$ (componente perpendicular) resultan ser proporcionales respectivamente a:

$$
\mu_{z}^{2}=\mu^{2} \cdot \cos ^{2} \theta \quad \text { y } \quad \mu_{x}^{2}=\mu^{2} \cdot \operatorname{sen}^{2} \theta \cdot \cos ^{2} \phi
$$

Sea $P(t)$ la probabilidad de encontrar al estado fluorescente aún excitado en el instante t. La contribución a la intensidad de la emisión total de los dipolos de transición, con esta orientación particular, será proporcional a

$$
\mu_{z}^{2} \cdot \mathbf{P}(\mathbf{t}) \quad y \quad \mu_{x}^{2} \cdot \mathbf{P}(\mathbf{t})
$$

Las intensidades totales se obtienen promediando sobre todas las orientaciones posibles en el espacio. Luego,

$$
\begin{aligned}
& \mathbf{I}_{z}(\mathbf{t})=\iint \operatorname{sen} \theta d \theta d \phi \cdot \mathbf{P}(\mathbf{t}) \cdot \mu^{2} \cos ^{2} \theta \cdot \mathbf{f}(\theta, \phi, t) \\
& \mathbf{I}_{x}(\mathbf{t})=\iint \operatorname{sen} \theta d \theta d \phi \cdot \mathbf{P}(\mathbf{t}) \cdot \mu^{2} \operatorname{sen}^{2} \theta \cos ^{2} \phi \cdot \mathbf{f}(\theta, \phi, t)
\end{aligned}
$$

Usando la expresión hallada para $f(\theta, \phi, t)$, el resultado de las integrales es como sigue:

$$
\begin{aligned}
& I_{z}(t)=\left[\frac{1}{3}+\frac{4}{15}\left\langle P_{2}(\mu(0) \cdot \mu(t))\right\rangle\right] \cdot P(t) \\
& I_{x}(t)=\left[\frac{1}{3}-\frac{2}{5}\left\langle P_{2}(\mu(0) \cdot \mu(t))\right\rangle\right] \cdot P(t)
\end{aligned}
$$

de donde la expresión para la anisotropía de la luminiscencia resulta,

$$
\mathbf{r}(\mathbf{t})=\frac{2}{5}\left\langle\mathbf{P}_{2}(\mu(0) \cdot \mu(t))\right\rangle
$$

Para hallar la dependencia explícita de la anisotropía con el tiempo, debemos buscar la solución a la ecuación de difusión, que ha de satisfacer la función de distribución de probabilidades ( $)$. 
En el caso particular de la difusión rotacional de una esfera de volumen $\mathrm{V}$ en un medio de viscosidad $\eta$, esta ecuación es:

$$
\frac{\partial \mathbf{f}(\theta, \phi, \mathbf{t})}{\partial \mathrm{t}}=\mathbf{D} \cdot \nabla^{2} \mathbf{f}
$$

siendo $\mathbf{D}$ el coeficiente de difusión rotacional, dado por $\quad \mathbf{6 D}=\frac{\mathbf{k T}}{\mathbf{V \eta}}$

Resolviendo la última ecuación en términos de las funciones de Green para la distribución de probabilidades, resulta:

$$
r(t)=\frac{2}{5} \cdot \exp ^{-6 D t}
$$

Para un rotor asimétrico, la ecuación de difusión puede ser escrita como [Favro]

$$
\frac{\partial \mathbf{f}(\Omega, \mathbf{t})}{\partial \mathbf{t}}=-\mathbf{H} \cdot \mathbf{f}(\Omega, \mathbf{t})
$$

estando el hamiltoniano dado por la siguiente expresión:

$$
\mathbf{H}=\sum_{\mathrm{ij}} \mathbf{L}_{\mathrm{i}} \cdot \mathbf{D}_{\mathrm{ij}} \cdot \mathbf{L}_{\mathrm{j}}
$$

$\mathbf{L}$ es el operador momento angular de la mecánica cuántica y $\mathbf{D}_{\mathrm{ij}}$ las componentes del tensor de difusión. Si se elige el sistema de coordenadas que diagonalice el tensor de difusión, entonces el hamiltoniano resulta

$$
\mathbf{H}=\sum_{\mathbf{i}} \mathbf{D}_{\mathbf{i}} \mathbf{L}_{\mathbf{i}}^{2}
$$

Ahora $\Omega$ en $\mathbf{f}(\Omega, \mathbf{t})$ se refiere a la orientación de un sistema de coordenadas fijo al cuerpo en rotación, respecto del sistema de coordenadas fijo en el espacio. $\Omega$ puede identificarse con los tres ángulos de Euler que rotan el sistema fijo al cuerpo en el sistema del laboratorio.

Una vez más se busca resolver la nueva ecuación de difusión por el método de las funciones de Green. 


$$
f(\Omega, t)=\int_{\Omega_{0}} f\left(\Omega_{0}\right) \cdot G\left(\Omega_{0} ; \Omega, t\right) d \Omega_{0}
$$

Haciendo uso de la analogía de este hamiltoniano con aquel operador de la mecánica cuántica para un rotor asimétrico, la función de Green puede ser expandida en términos de las autofunciones de este último operador,

$$
G\left(\Omega_{0} ; \Omega, t\right)=\sum_{n} \psi_{n}^{*}\left(\Omega_{0}\right) \cdot \psi_{n}(\Omega) \cdot \operatorname{Exp}\left(-\mathbf{E}_{n} t\right)
$$

que cumplen con la condición inicial:

$$
G\left(\Omega_{0} ; \Omega, 0\right)=\delta\left(\Omega_{0}, \Omega\right)=\sum_{n} \psi_{n}^{*}\left(\Omega_{0}\right) \cdot \psi_{n}(\Omega)
$$

Sean $x, y, z$, los ejes principales del tensor de difusión. Estos se pueden relacionar con los ejes fijos en el sistema del laboratorio mediante la siguiente transformación : $\mathbf{X}=\mathbf{R} . \mathbf{x}$, donde $\mathbf{R}$ es una matriz que realiza la rotación.

Antes habiamos supuesto que los dipolos de absorción y emisión eran paralelos. Consideremos ahora el caso más general. Sean $\mathrm{h}_{\mathrm{i}}$ las projecciones del vector unitario del dipolo de absorción a lo largo de los ejes moleculares y $\mathrm{g}_{\mathrm{i}}$ las correspondientes componentes del dipolo de emisión. Entonces la probabilidad de que una molécula sea excitada es ahora,

$$
P_{\text {abs. }}=\left(h_{x} R_{Z x}+h_{y} R_{Z y}+h_{z} R_{Z z}\right)^{2}
$$

donde $\mathbf{R}_{\mathbf{i j}}$ es el elemento de la matriz de rotación y $\sum_{\mathbf{i}} \mathbf{h}_{\mathbf{i}}^{2}=\mathbf{1}$.

Las intensidades de emisión con polarización paralela y perpendicular a la excitación, son proporcionales respectivamente a

$$
\begin{aligned}
& \mathbf{P}_{\mathrm{em} .}^{z}=\left(g_{x} R_{Z x}+g_{y} R_{Z y}+g_{z} R_{Z z}\right)^{2} \\
& P_{e m .}^{x}=\left(g_{x} R_{X x}+g_{y} R_{X y}+g_{z} R_{X z}\right)^{2}
\end{aligned}
$$

Las intensidades de emisión, observadas en la dirección $\mathrm{Y}$, con polarización paralela y perpendicular son entonces:

$$
I_{z}(t)=\int_{\Omega} P_{c m}^{z} \cdot P(t) \cdot f(\Omega, t) d \Omega
$$




$$
I_{x}(t)=\int_{\Omega} P_{c m}^{x} \cdot P(t) \cdot f(\Omega, t) d \Omega
$$

Si la emisión de las moléculas fluorescentes decae exponencialmente con un tiempo de vida $\tau$, entonces $P(t)=\exp (-t / \tau)$. Luego, resolviendo las integrales (ver apéndice $A$ ) la expresión general para la anisitropía puede ser escrita como sigue,

$$
r(t)=\sum_{i=1}^{5} A_{i} \cdot \operatorname{Exp}[- \text { ai. } t]
$$

donde $\mathbf{a i}^{-1}$ son los tiempos de correlación rotacional; y la anisotropía límite de la emisión, $\mathrm{r}_{\mathrm{O}}$, está determinada por la suma de los factores preexponenciales Ai. Los ai contienen los coeficientes de difusión para la rotación relativa a los ejes inerciales.

La anisotropía de la polarización en estado estacionario se obtiene tomando el promedio temporal sobre el decaimiento de la emisión

$$
\begin{aligned}
& \overline{\mathbf{I}}_{z}=\frac{1}{\tau} \cdot \int_{0}^{\infty} I_{z}(t) d t \\
& \overline{\mathbf{I}}_{x}=\frac{1}{\tau} \cdot \int_{0}^{\infty} I_{x}(t) d t
\end{aligned}
$$

Luego la expresión que resulta para la anisotropía en estado estacionario es,

$$
\overline{\mathbf{r}}=\sum_{\mathbf{i}=1}^{5} \frac{\mathbf{A}_{\mathbf{i}}}{1+\mathbf{a}_{i} \cdot \tau}
$$

donde $\tau$ es el tiempo de vida del estado fluorescente

Se tienen así expresiones generales para la depolarización de la fluorescencia, dependiente del tiempo y en estado estacionario. En la evolución temporal de la anisotropía aparecen cinco decaimientos exponenciales, sin contar el decaimiento propio de la luminiscencia con $\tau$. Los resultados de Weber quedan contemplados como caso particular, debido a que él asume un dipolo de absorción sobre uno de los ejes moleculares. Este claramente no es el caso más general, dado que el dipolo puede en principio tener cualquier dirección en el sistema molecular La expresión de Tao de la depolarización de la luminiscencia, tiene errores debido a la proyección incorrecta del dipolo de absorción a lo largo de los ejes moleculares. La importancia de contar con la expresión correcta de la 
depolarización de la luminiscencia está en el hecho de que las cantidades fisicas de interés, los coeficientes de difusión rotacional $\left(D_{\mathrm{i}}\right)$, son deducidos a partir de la anisotropía de la fluorescencia observada experimentalmente. Estas expresiones son esenciales para obtener información adicional acerca de las proyecciones de los dipolos de absorción y de emisión, a lo largo de los ejes principales de difusión.

\subsubsection{EXPRESIONES PARTICULARES DE LA ANISOTROPÍA.}

Analicemos ahora algunos casos de interés particular. Supongamos que la molécula pueda ser modelada con un elipsoide oblado. Entonces la molécula posee un eje de simetría perpendicular al plano que la contiene. El coeficiente de difusión rotacional para el movimiento en el plano de la molécula $\left(D_{p}\right)$, será diferente de los coeficientes de rotación alrededor de los ejes contenidos en dicho plano. Es evidente además, que la existencia de un eje de simetría hace que los coeficientes de difusión ecuatoriales, perpendiculares a dicho eje, sean indistinguibles. Así se tendrán dos únicas constantes de difusión, $\mathrm{D}_{\mathrm{s}}$ y $\mathrm{D}_{\mathrm{p}}$, que están relacionadas por las leyes hidrodinámicas con los semiejes del elipsoide. Una simplificación adicional, bastante intuitiva también, consiste en suponer que el dipolo de transición es perpendicular al eje de simetria, vale decir que está contenido en el plano molecular. En este caso resulta para la anisotropía de la fluorescencia dependiente del tiempo, la siguiente expresión,

$$
r(t)=r_{o} \cdot\left[\frac{1}{4} \exp -\left(6 D_{s}\right) t+\frac{3}{4} \exp -\left(2 D_{s}+4 D_{p}\right) t\right]
$$

Normalmente estos dos decaimientos exponenciales son muy similares [ver tabla II ref. Tau], y entonces el decaimiento de la anisotropía puede describirse en forma aproximada mediante una sola exponencial. Podemos mencionar a modo de ejemplo el caso del colorante rose bengal [Spears], cuya forma molecular se modela con un elipsoide oblado de semiejes 2 y $7 \AA$. La diferencia entre los coeficientes $6 \mathrm{D}_{\mathrm{s}}$ y $\left(2 \mathrm{D}_{\mathrm{s}}+4 \mathrm{D}_{\mathrm{p}}\right)$ es del orden del $15 \%$. Este colorante rota casi isotrópicamente con un decaimiento que puede obtenerse haciendo el promedio de aquellos dos coeficientes.

Un sólo decaimiento exponencial es el que corresponde a una molécula esférica. De todos los casos éste es el más simple. Dado que la difusión es isotrópica, el decaimiento de la anisotropía puede estar caracterizado por una única constante de difusión. La rotación 
alrededor de un eje cualquiera tiene la misma constante de difusión. Luego en la expresión de arriba $D_{s}=D_{p}$. Así para una molécula esférica,

$$
r(t)=r_{0} \cdot \exp -(6 D t)
$$

y en estado estacionario,

$$
\begin{aligned}
& \bar{r}=\frac{1}{\tau} \cdot \int_{0}^{\infty} \exp -(t / \tau) \cdot \mathbf{r}(t) \cdot d t \\
& \frac{\mathbf{r}_{0}}{\mathbf{r}}-1=6 \mathrm{D} \tau
\end{aligned}
$$

que es la bien conocida ecuación de Perrin.

Es fácil ver además que esta expresión es válida también para un elipsoide prolado con el dipolo de transición a lo largo de su eje mayor (eje de simetría molecular). Si z es este eje, entonces $h_{i}=g_{i}=\delta_{i z}$. Luego (ver apéndice $A$ ),

$$
\begin{aligned}
& \mathrm{S}=2 \mathrm{~d}_{\mathrm{x}}+\mathrm{d}_{\mathrm{z}} \\
& \mathrm{U}^{2}=\left(\mathrm{d}_{\mathrm{x}}-\mathrm{d}_{\mathrm{z}}\right)^{2} \\
& \lambda=2 / 3 \\
& \omega=2 / 3
\end{aligned}
$$

Reemplazando en la expresión de la anisotropía, resulta:

$$
\begin{aligned}
& r(t)=3 / 10 \cdot(2 / 3+2 / 3) \cdot \exp -\left[2\left(3 d_{x}\right) D t\right] \\
& r(t)=0.4 \exp -\left(6 D_{x} t\right) \\
& \text { donde } D_{x}=D \cdot d_{x}
\end{aligned}
$$

Como resultado de estos tres ejemplos que acabamos de ver, podemos concluir que sin más información, como puede ser el volumen del cuerpo en rotación, una curva de despolarización de la fluorescencia que sea sólo una exponencial, no puede ser distinguida de entre los siguientes casos: geometría esférica, geometría oblada o prolada con un volumen mayor y una orientación preferencial del dipolo de la transición. 


\subsubsection{ANISOTROPÍA LÍMITE.}

En los ejemplos que acabo de considerar he supuesto que el dipolo de absorción y el de emisión son colineales. En este caso particular es fácil ver que el valor de la anisotropía limite $\left(r_{0}\right)$, es 0.4

Consideremos una molécula cuyo momento dipolar forma un ángulo $\theta$ con el vector eléctrico de la radiación incidente (fig. 3.1). La energía que entrega la radiación al dipolo $\mu$ es proporcional al $\cos ^{2} \theta$ ( la probabilidad de excitación de la molécula es $\cos ^{2} \theta$ ).

En ausencia de movimiento Browniano, con osciladores lineales ligados rígidamente a las moléculas, las intensidades radiadas tendrán componentes proporcionales a :

$$
\begin{aligned}
& \mathrm{I}_{\mathrm{x}} \sim \operatorname{sen}^{2} \theta \cdot \cos ^{2} \phi \\
& \mathrm{I}_{\mathrm{y}} \sim \operatorname{sen}^{2} \theta \cdot \operatorname{sen}^{2} \phi \\
& \mathrm{I}_{\mathrm{z}} \sim \cos ^{2} \theta
\end{aligned}
$$

dado que las componentes del dipolo no cambian por estár éste fijo en el espacio.

Si las moléculas están distribuidas con orientaciones al azar dentro de la solución, el número de moléculas excitadas con momento dipolar en la dirección $(\theta, \phi)$ más $(\mathrm{d} \theta, \mathrm{d} \phi)$ es:

$$
\cos ^{2} \theta \cdot \operatorname{sen} \theta \cdot d \theta \cdot d \phi
$$

Luego las intensidades totales observadas en la dirección $\mathrm{Y}$ con polarización en las direcciones X y $Z$, serán:

$$
\begin{aligned}
& I_{x}=C \cdot \int_{0}^{\pi} \int_{0}^{2 \pi} \operatorname{sen} \theta \cdot d \theta \cdot d \phi \cdot \cos ^{2} \theta \cdot \operatorname{sen}^{2} \theta \cdot \cos ^{2} \phi \\
& I_{z}=C \cdot \int_{0}^{\pi} \int_{0}^{2 \pi} \operatorname{sen} \theta \cdot d \theta \cdot d \phi \cdot \cos ^{2} \theta \cdot \cos ^{2} \theta
\end{aligned}
$$

donde $\mathrm{C}$ es una constante.

Resolviendo las integrales se obtiene,

$$
\mathrm{I}_{\mathrm{x}}=\mathrm{C} .1 / 15 \quad \mathrm{e} \quad \mathrm{I}_{\mathrm{z}}=\mathrm{C} \cdot 3 / 15
$$

Con lo cual, $r_{n}=2 / 5$. 
Este valor es dificil de encontrar en la práctica, a causa de alguna despolarización que resulta de factores externos tales como pueden ser tensiones en la matriz rígida que contiene la muestra [Paladini]. Desplazamientos rápidos del dipolo de la transición, alrededor de su posición media dentro de la molécula, podrían causar también una disminución de la anisotropía máxima que podemos observar [Perrin, 1929].

Supongamos ahora que el dipolo de absorción y el de emisión están fijos en la molécula, pero forman un ángulo $\alpha$ entre sí. La transferencia de activación del primer dipolo al de emisión equivale a un desplazamiento angular medio de todas las posiciones moleculares correspondientes a una misma dirección del dipolo de absorción, alrededor de aquella posición. Resulta así,

$$
r_{0}=\frac{3 \cos ^{2} \alpha-1}{5}
$$

Dado que $\alpha$ puede variar entre 0 y $\pi / 2$, la anisotropía límite puede adoptar cualquier valor entre

$$
-0.2 \leq \mathrm{r}_{\mathrm{o}} \leq 0.4
$$

Resulta interesante observar también que si $\alpha=54.74^{\circ}$, la anisotropia en ausencia de movimiento Browniano es cero; con lo cual las componentes con polarización paralela y perpendicular de la emisión, tienen la misma intensidad durante todo el proceso de decaimiento radiativo de las moléculas

Dependiendo del ángulo que formen el dipolo de absorción y el de emisión, tal como acabamos de ver, el valor $r_{0}$ puede resultar negativo. Un valor negativo de la anisotropía, significa que la luminiscencia emitida perpendicularmente al vector eléctrico de la onda incidente tiene su máximo de vibración eléctrica en el plano perpendicular a este vector eléctrico de excitación. 


\subsection{CÁLCULO DE LOS COEFICIENTES DE DIFUSIÓN ROTACIONAL}

Los coeficientes de difusión rotacional pueden ser evaluados acorde a las ecuaciones hidrodinámicas de Perrin (1934). Estos coeficientes son funciones de la forma y tamaño de la molécula luminiscente, como así también de la viscosidad y temperatura del solvente. Sus expresiones en el caso más general se verán en la siguiente sección (3.3.1). Para el caso particular de elipsoides de revolución, resulta :

$$
\begin{aligned}
& \mathbf{d}_{\mathrm{p}}=\frac{3 \cdot \rho \cdot\left(\rho-\mathbf{S}^{\prime}\right)}{2 \cdot\left(\rho^{2}-1\right)} \\
& \mathbf{d}_{\mathrm{s}}=\frac{3 \cdot \rho \cdot\left[\left(2 \rho^{2}-1\right) \cdot \mathrm{S}^{\prime}-\rho\right]}{2 \cdot\left(\rho^{4}-1\right)}
\end{aligned}
$$

donde $\rho$ es el cociente entre el eje de revolución y el eje ecuatorial; menor que uno para un rotor oblado (con forma de disco) y mayor que uno para un rotor prolado (con forma de vara). El parámetro $S^{\prime}$ se define dependiendo del valor de $\rho$ como sigue:

$$
\begin{array}{ll}
S^{\prime}=\frac{1}{\sqrt{\rho^{2}-1}} \cdot \ln \left(\rho+\sqrt{\rho^{2}-1}\right) & \rho>1 \\
S^{\prime}=\frac{1}{\sqrt{1-\rho^{2}}} \cdot \operatorname{arctg}\left(\frac{\sqrt{1-\rho^{2}}}{\rho}\right) & \rho<1 \quad \text { (prolado) }
\end{array}
$$

Los tiempos de relajación rotacional pueden ser entonces evaluados a partir de estos dos coeficientes de difusión adimensionales. En la figura 3.2 se los ha representado como función de $\rho$, para elipsoides prolados y oblados $(\rho=1$ corresponde a la geometría esférica) Puede verse claramente como para elipsoides prolados estos coeficientes se diferencian uno de otro rápidamente al aumentar $\rho$, mientras que para un elipsoide oblado esta diferencia no se hace tan importante. 


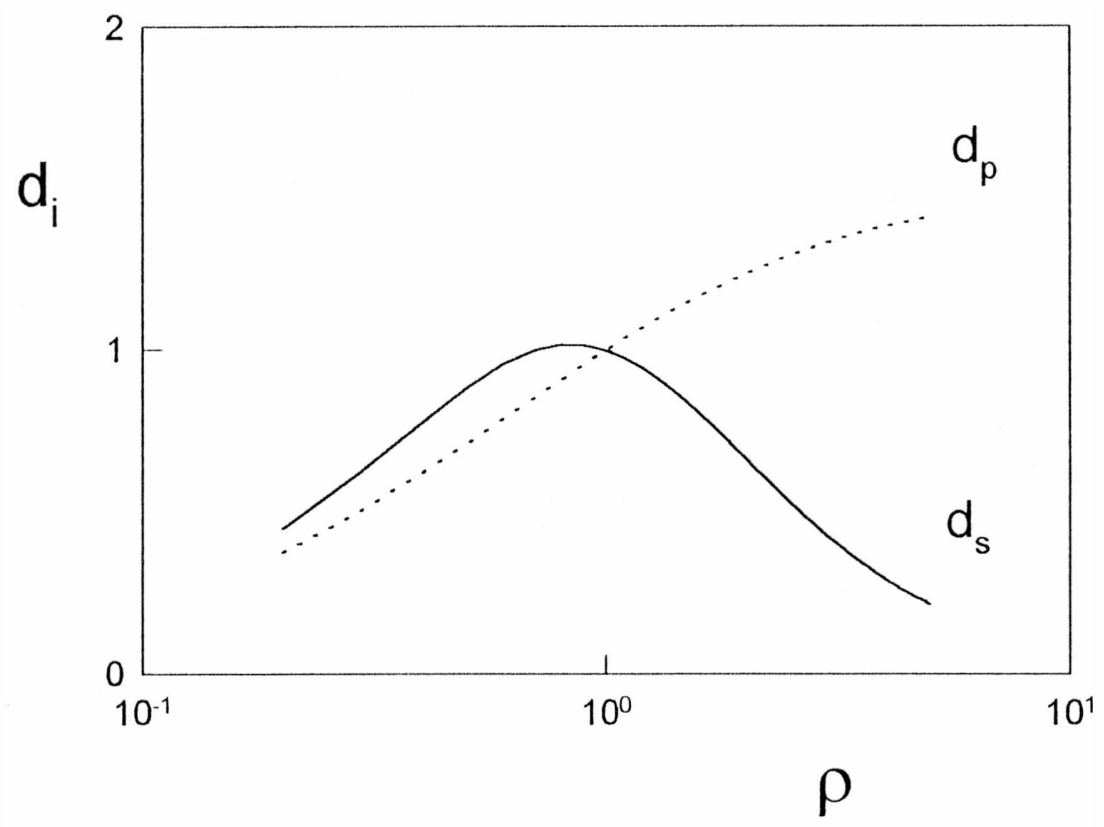

Figura 3.2. Coeficientes de difusión rotacional (adimensionales) de un elipsoide simétrico. $d_{p}$ es el coeficiente de difusión para la rotación alrededor del eje de revolución del elipsoide $y d_{s}$ el correspondiente al eje ecuatorial.

Dado que los colorantes son moléculas planas, puede resultar no ser una buena aproximación considerar elipsoides prolados para modelarlas. Debemos tratar entonces con elipsoides asimétricos para simular la forma de las moléculas. Esto trae aparejado un problema complejo, en el sentido de tener que evaluar los coeficientes de difusión rotacional alrededor de cada eje del elipsoide. Si bien ello ha sido llevado adelante por Perrin, usando la aproximación hidrodinámica de Stokes, la evaluación de los mismos requiere resolver ecuaciones elípticas, para las cuales, salvo en el caso particular de los elipsoides de revolución que acabamos de considerar, no existe solución analítica. Así, en la obtención de la expresión correspondiente a la anisotropía de un elipsoide asimétrico, deberemos primero establecer los semiejes del elipsoide mediante un modelado acorde a la estructura de la molécula y después evaluar numéricamente las integrales que permitan conocer los coeficientes de difusión rotacional 


\subsubsection{COEFICIENTES DE DIFUSIÓN ROTACIONAL PARA UN ELIPSOIDE ASIMÉTRICO}

Consideremos entonces ahora el caso más general. Este ha sido tratado en primer lugar por Edwardes y posteriormente, como ya fuera mencionado, por Perrin. Sean $x, y, z$, las direcciones correspondientes a los tres ejes de simetría del elipsoide que representa a la molécula; a,b,c, las longitudes de los semiejes en aquellas direcciones, respectivamente. El coeficiente de difusión rotacional alrededor del eje $i$, es:

$$
\mathrm{D}_{i}=\mathrm{k} \cdot \mathrm{T} / \mathrm{C}_{i}
$$

donde $\mathrm{k}$ es la constante de Boltzmann, $\mathrm{T}$ la temperatura absoluta y $\mathrm{C}_{i}$ es el coeficiente de fricción para la rotación alrededor del eje $i$. Estos coeficientes están dados por las siguientes expresiones [Woessner],

$$
\begin{aligned}
& \mathrm{C}_{\mathrm{x}}=16 \cdot \pi \cdot \eta \cdot\left(\mathrm{b}^{2}+\mathrm{c}^{2}\right) /\left[3 \cdot\left(\mathrm{b}^{2} \cdot \mathrm{Q}+\mathrm{c}^{2} \cdot \mathrm{R}\right)\right], \\
& \mathrm{C}_{\mathrm{y}}=16 \cdot \pi \cdot \eta \cdot\left(\mathrm{a}^{2}+\mathrm{c}^{2}\right) /\left[3 \cdot\left(\mathrm{a}^{2} \cdot \mathrm{P}+\mathrm{c}^{2} \cdot \mathrm{R}\right)\right], \\
& \mathrm{C}_{\mathrm{z}}=16 \cdot \pi \cdot \eta \cdot\left(\mathrm{a}^{2}+\mathrm{b}^{2}\right) /\left[3 \cdot\left(\mathrm{a}^{2} \cdot \mathrm{P}+\mathrm{b}^{2} \cdot \mathrm{Q}\right)\right],
\end{aligned}
$$

donde $\eta$ es la viscosidad del solvente a temperatura T, y P, Q y R son integrales elípticas de la forma:

$$
\begin{aligned}
& P=\int_{0}^{\infty}\left[\left(a^{2}+s\right)^{3} \cdot\left(b^{2}+s\right) \cdot\left(c^{2}+s\right)\right]^{-1 / 2} d s, \\
& Q=\int_{0}^{\infty}\left[\left(a^{2}+s\right) \cdot\left(b^{2}+s\right)^{3} \cdot\left(c^{2}+s\right)\right]^{-1 / 2} d s, \\
& R=\int_{0}^{\infty}\left[\left(a^{2}+s\right) \cdot\left(b^{2}+s\right) \cdot\left(c^{2}+s\right)^{3}\right]^{-1 / 2} d s,
\end{aligned}
$$

Estas cantidades se relacionan de la siguiente manera:

$$
P+Q+R=2 /\left(\text { a.b.c) } \quad y \quad a^{2} \cdot P+b^{2} \cdot Q+c^{2} \cdot R=W\right.
$$


donde $W=\int_{0}^{\infty}\left[\left(a^{2}+s\right) \cdot\left(b^{2}+s\right) \cdot\left(c^{2}+s\right)\right]^{-1 / 2} d s$

Con el siguiente cambio de la variable de integración, logramos adimensionalizar las integrales, posibilitando así su posterior evaluación por métodos numéricos:

$$
\mathrm{u}=\mathrm{s} / \mathrm{a}^{2} \quad \mathrm{ds}=\mathrm{a}^{2} \cdot \mathrm{du}
$$

Ahora las tres integrales pueden volver a escribirse como sigue:

$$
\begin{aligned}
& \mathrm{P}=(\mathrm{a} \cdot \mathrm{b} \cdot \mathrm{c})^{-1} \cdot \int_{0}^{\infty}\left[(1+\mathrm{u})^{3} \cdot\left(1+\alpha^{2} \cdot \mathrm{u}\right) \cdot\left(1+\beta^{2} \cdot \mathrm{u}\right)\right]^{-1 / 2} \mathrm{du}, \\
& \mathrm{Q}=\mathrm{a} /\left(\mathrm{b}^{3} \cdot \mathrm{c}\right) \cdot \int_{0}^{\infty}\left[(1+\mathrm{u}) \cdot\left(1+\alpha^{2} \cdot \mathrm{u}\right)^{3} \cdot\left(1+\beta^{2} \cdot \mathrm{u}\right)\right]^{-1 / 2} \mathrm{du}, \\
& \mathrm{R}=\mathrm{a} /\left(\mathrm{b} \cdot \mathrm{c}^{3}\right) \cdot \int_{0}^{\infty}\left[(1+\mathrm{u}) \cdot\left(1+\alpha^{2} \cdot \mathrm{u}\right) \cdot\left(1+\beta^{2} \cdot \mathrm{u}\right)^{3}\right]^{-1 / 2} \mathrm{du}, \\
& \text { donde } \quad \alpha=\mathrm{a} / \mathrm{b} \quad \mathrm{y} \quad \beta=\mathrm{a} / \mathrm{c} .
\end{aligned}
$$

Bajo las mismas condiciones, el coeficiente de difusión rotacional de una molécula esférica con el mismo volumen que el elipsoide ( $V=4 / 3 . \pi$.a.b.c $)$ es $D=k . T / 6 . V . \eta$.

Definiendo $d_{i}=D_{i} / D$, puede verse facilmente que en términos de $p, q$ y $r$; donde

$$
\begin{aligned}
& \mathrm{p}=\text { P.a.b.c } \quad \mathrm{q}=\mathrm{Q} \cdot \mathrm{b}^{3} \cdot \mathrm{c} / \mathrm{a} \quad \mathrm{r}=\mathrm{R} \cdot \mathrm{c}^{3} \cdot \mathrm{b} / \mathrm{a} \\
& \mathrm{d}_{\mathrm{x}}=[3 \cdot(\mathrm{q}+\mathrm{r})] /\left[2 \cdot\left(\alpha^{-2}+\beta^{-2}\right)\right], \\
& \mathrm{d}_{\mathrm{y}}=[3 \cdot(\mathrm{r}+\mathrm{p})] /\left[2 \cdot\left(1+\beta^{-2}\right)\right], \\
& \mathrm{d}_{\mathrm{z}}=[3 \cdot(\mathrm{p}+\mathrm{q})] /\left[2 \cdot\left(1+\alpha^{-2}\right)\right],
\end{aligned}
$$


Dados los semiejes del elipsoide, los coeficientes de difusión rotacional adimensionales $\left(d_{i}\right)$ pueden ser obtenidos evaluando las integrales elipticas ( $p, q$ y $r$ )

\subsubsection{MODELOS HIDRODINÁMICOS ( STICK - SLIP )}

En el cálculo de los coeficientes de difusión rotacional alrededor de cada eje del elipsoide, Perrin emplea las ecuaciones de Navier-Stokes. Usando la aproximación hidrodinámica de Stokes calcula el torque que actúa contra la rotación de un cuerpo inmerso en un fluido continuo. Se supone además que en la superficie del cuerpo, el fluido en contacto con éste sigue solidariamente su movimiento y esta condición de contorno soluto-solvente, subyacente en el cálculo de los coeficientes de fricción, se conoce como condición de contorno stick

Los coeficientes de difusión rotacional así obtenidos, se corresponden muy bien con aquellos hallados experimentalmente en el caso de moléculas grandes, tales como macromoléculas biológicas (por ej. proteínas o polímeros). Pero para moléculas pequeñas en soluciones líquidas, los tiempos de relajación rotacional que se determinan experimentalmente resultan ser frecuentemente mucho más pequeños que aquellos que son calculados sobre la base de la condición de contorno stick. En general, esta condición es válida para objetos grandes macroscópicamente. Cuando la misma se aplica a sistemas en los que tanto el soluto como el solvente son de dimensiones moleculares, aparecen discrepancias entre este modelo hidrodinámico y el experimento. En estos casos resulta más apropiado el uso de la condición de contorno slip. De acuerdo con este modelo, la componente tangencial del tensor de esfuerzos sobre la superficie del cuerpo en rotación es nula, sin embargo algo de fluido será desplazado cuando el objeto rota (si su forma no es esférica) dado que el fluido no puede penetrar ni abandonar la superficie del cuerpo (ver apéndice B). La condición de contorno slip marca una diferencia importante con stick para el movimiento rotacional. Hu y Zwanzig fueron quienes introdujeron la condición de contorno slip y obtuvieron soluciones numéricas ( no hay una solución exacta al problema de contorno slip ) para los coeficientes de fricción de distintos esferoides. Ellos resuelven la ecuación de Navier-Stokes para la distribución de velocidades de un fluido viscoso, alrededor de un esferoide (elipsoide prolado u oblado) que está rotando uniformemente con una dada velocidad angular, con condición de contorno slip sobre la superficie del esferoide. Usando esta distribución de velocidades calcularon el torque hidrodinámico sobre el esferoide.

Posteriormente, Youngren y Acrivos dedujeron una aproximación diferente para obtener los valores numéricos de los coeficientes de fricción de elipsoides asimétricos, rotando alrededor de sus ejes principales, bajo condición de contorno slip. Sus resultados 
contemplan como caso particular el de Hu y Zwanzig, si bien un error en la presentación de los mismos (tabla 1, ref. Youngren) podría causar una mala interpretación de medidas experimentales. En la tabla se dice que son presentados los coeficientes de fricción adimensionales, cuando en realidad son indicados a menos de un factor $V / \pi$, donde $V$ es el volumen del elipsoide. Este error fue también notado por Sension y Hochstrasser, y los resultados corregidos se muestran en la tabla 3.1 .

Tabla 3.1. Coeficientes de fricción adimensionales ( $\zeta$ ), con condición slip. a y b son las longitudes de los semiejes del elipsoide en las direcciones y y $\mathrm{z}$ respectivamente. En $\mathrm{x}$ la longitud del semieje tiene valor uno.

\begin{tabular}{|c|c|c|c|c|c|c|c|c|c|}
\hline bla & 1.0 & 0.9 & 0.8 & 0.7 & 0.6 & 0.5 & 0.4 & 0.3 & 0.2 \\
\hline 1.0 & $\begin{array}{l}0.00 \\
0.00 \\
0.00\end{array}$ & & & & & & & & \\
\hline 0.9 & $\begin{array}{l}0.04 \\
0.04 \\
0.00\end{array}$ & $\begin{array}{l}0.00 \\
0.05 \\
0.05\end{array}$ & & & & & & & \\
\hline 0.8 & $\begin{array}{l}0.19 \\
0.19 \\
0.00\end{array}$ & $\begin{array}{l}0.05 \\
0.20 \\
0.04\end{array}$ & $\begin{array}{l}0.00 \\
0.20 \\
0.20\end{array}$ & & & & & $\begin{array}{l}\zeta_{\mathrm{x}} \\
\zeta_{\mathrm{y}} \\
\zeta_{z}\end{array}$ & \\
\hline 0.7 & $\begin{array}{l}0.47 \\
0.47 \\
0.00\end{array}$ & $\begin{array}{l}0.23 \\
0.49 \\
0.05\end{array}$ & $\begin{array}{l}0.07 \\
0.51 \\
0.21\end{array}$ & $\begin{array}{l}0.00 \\
0.54 \\
0.54\end{array}$ & & & & & \\
\hline 0.6 & $\begin{array}{l}0.96 \\
0.96 \\
0.00\end{array}$ & $\begin{array}{l}0.57 \\
0.99 \\
0.06\end{array}$ & $\begin{array}{l}0.28 \\
1.02 \\
0.23\end{array}$ & $\begin{array}{l}0.09 \\
1.07 \\
0.57\end{array}$ & $\begin{array}{l}0.00 \\
1.13 \\
1.13\end{array}$ & & & & \\
\hline 0.5 & $\begin{array}{l}1.77 \\
1.77 \\
0.00\end{array}$ & $\begin{array}{l}1.22 \\
1.80 \\
0.07\end{array}$ & $\begin{array}{l}0.75 \\
1.86 \\
0.26\end{array}$ & $\begin{array}{l}0.36 \\
1.95 \\
0.62\end{array}$ & $\begin{array}{l}0.10 \\
2.00 \\
1.25\end{array}$ & $\begin{array}{l}0.00 \\
2.25 \\
2.25\end{array}$ & & & \\
\hline 0.4 & $\begin{array}{l}3.13 \\
3.13 \\
0.00\end{array}$ & $\begin{array}{l}2.42 \\
3.19 \\
0.06\end{array}$ & $\begin{array}{l}1.69 \\
3.26 \\
0.28\end{array}$ & $\begin{array}{l}1.04 \\
3.38 \\
0.67\end{array}$ & $\begin{array}{l}0.53 \\
3.53 \\
1.41\end{array}$ & $\begin{array}{l}0.15 \\
3.79 \\
2.48\end{array}$ & $\begin{array}{l}0.00 \\
4.17 \\
4.17\end{array}$ & & \\
\hline 0.3 & $\begin{array}{l}5.45 \\
5.45 \\
0.00\end{array}$ & $\begin{array}{l}4.44 \\
5.56 \\
0.08\end{array}$ & $\begin{array}{l}3.44 \\
5.69 \\
0.31\end{array}$ & $\begin{array}{l}2.46 \\
5.89 \\
0.75\end{array}$ & $\begin{array}{l}1.58 \\
6.21 \\
1.58\end{array}$ & $\begin{array}{l}0.85 \\
6.60 \\
2.80\end{array}$ & $\begin{array}{l}0.25 \\
7.19 \\
4.81\end{array}$ & $\begin{array}{l}0.00 \\
8.17 \\
8.17\end{array}$ & \\
\hline 0.2 & $\begin{array}{l}10.1 \\
10.1 \\
0.00\end{array}$ & $\begin{array}{l}8.54 \\
10.4 \\
0.13\end{array}$ & $\begin{array}{l}7.03 \\
10.7 \\
0.37\end{array}$ & $\begin{array}{l}5.52 \\
11.0 \\
0.91\end{array}$ & $\begin{array}{l}4.13 \\
11.6 \\
1.88\end{array}$ & $\begin{array}{l}2.70 \\
12.3 \\
3.45\end{array}$ & $\begin{array}{l}1.50 \\
13.4 \\
5.91\end{array}$ & $\begin{array}{l}0.50 \\
15.2 \\
10.0\end{array}$ & $\begin{array}{l}0.00 \\
18.6 \\
18.6\end{array}$ \\
\hline
\end{tabular}


En la tabla aparecen tabulados los coeficientes de fricción adimensionales $\left(\zeta_{\mathrm{i}}=6 / \mathrm{d}_{\mathrm{i}}\right)$ para los elipsoides $x^{2}+y^{2} / a^{2}+z^{2} / b^{2}=1$, rotando alrededor de cada uno de sus ejes principales. Los resultados que aparecen en la columna de la izquierda y a lo largo de la diagonal concuerdan muy bien con las soluciones dadas por Hu y Zwanzig para esferoides oblados y prolados respectivamente

Los coeficientes principales de difusión rotacional de elipsoides asimétricos pueden entonces ser obtenidos en cualquiera de las dos aproximaciones hidrodinámicas al problema de condiciones de contorno (stick-slip). Con estos coeficientes y conociendo la orientación, respecto de los ejes de simetría molecular, de los dipolos de transición para absorción y emisión, se construye la expresión particular para la anisotropía de la fluorescencia de la molécula de colorante bajo estudio. 


\section{CAPítulo 4. CARACTERIZACIÓN FOTOFísiCa de las CIANINAS ESTUDIADAS.}

\subsection{INTRODUCCIÓN}

Es bien sabido que la excitación con luz de muchas cianinas normalmente produce una especie nueva. Esta especie transiente, denominada fotoisómero P, relaja en forma casi reversible a la forma normal, N. Los tiempos de vida medidos de distintos fotoisómeros en su estado fundamental, cubren varios ordenes de magnitud. Se han hallado fotoisómeros de vida corta, del orden de los nanosegundos, hasta aquellos con tiempos de vida del microsegundo o milisegundo [Tocho]. Para cada compuesto, los tiempos de vida y otros parámetros espectroscópicos, dependen fuertemente de la temperatura y de las propiedades del solvente.

La formación de especies fotoisoméricas ha sido demostrada a través de extensos estudios experimentales. Entre otras técnicas se usaron, flash fotólisis [Dempster], fluorescencia [Arthurs (1973), Scaffardi], absorción transiente [Knudtson] y espectroscopía de picosegundos resuelta en tiempo [Sundström, Sibbett]. A pesar de la gran cantidad de trabajo realizado, la información disponible para modelar estos sistemas está reducida a la isomerización $\mathrm{N} \rightarrow \mathrm{P}$, a través del primer estado singlete excitado de la forma normal, $S_{1}^{N}$, mientras que la retroisomerización $\mathrm{P} \rightarrow \mathrm{N}$ es sólo considerada arrancando desde el estado fundamental de la especie transiente, $\mathrm{S}_{0}^{\prime}$. Resulta escasa la información disponible sobre eficiencia cuántica de fotoisomerización de muchas de cianinas. Las propiedades de $S_{1}^{p}$, el primer estado excitado de $\mathrm{P}$, y su participación en el proceso de retroisomerización han sido aún menos estudiadas. Más adelante se verá como la irradiación con alta fluencia, usando láseres pulsados o continuos, permite obtener una población relativamente alta de $\mathrm{P}$ y analizar sus propiedades ópticas. Como señalara Momicchioli, la existencia de modelos teóricos dando cuenta de la fotoisomerización parecen estar menos desarrollados. Rulliere, siguiendo el modelo propuesto por Orlandi y Siebrand para la fotoisomerización del estilbeno, fue el primero en sugerir una curva de energía potencial para describir la isomerización del DODCI. Desde el estado excitado $S_{1}^{N}$ la molécula cae por relajación activada a un estado twisted, desde el cual el decaimiento continua muy rápidamente al estado fundamental, ya sea de la forma normal o del fotoisómero. El estado intermedio tiene un tiempo de vida demasiado corto para ser detectado directamente. Para explicar la isomerización de los colorantes tri y pentametino, Dietz por su parte sugiere un modelo que incluye la existencia de un mínimo adicional en la superficie $S_{0}$, producido en principio por la inclusión de efectos de solvatación en los cálculos. 


\subsection{DESCRIPCIÓN DEL MODELO CINÉTICO}

Uno de los problemas que afectan el desarrollo de un modelo completo, es que en general existe muy poca información acerca de las propicdades de los fotoisómeros. A los efectos de analizar en detalle la dinámica de isomerización de las cianinas estudiadas, haremos una descripción completa del modelo usado para representar los procesos que tienen lugar cuando estas moléculas son irradiadas. La figura 4.1 muestra un esquema simplificado de niveles de energía con conexiones entre isómeros, que se corresponde con el modelo desarrollado por Rullière, basado en la existencia de dos formas isoméricas: normal (N) y fotoisómero (P).

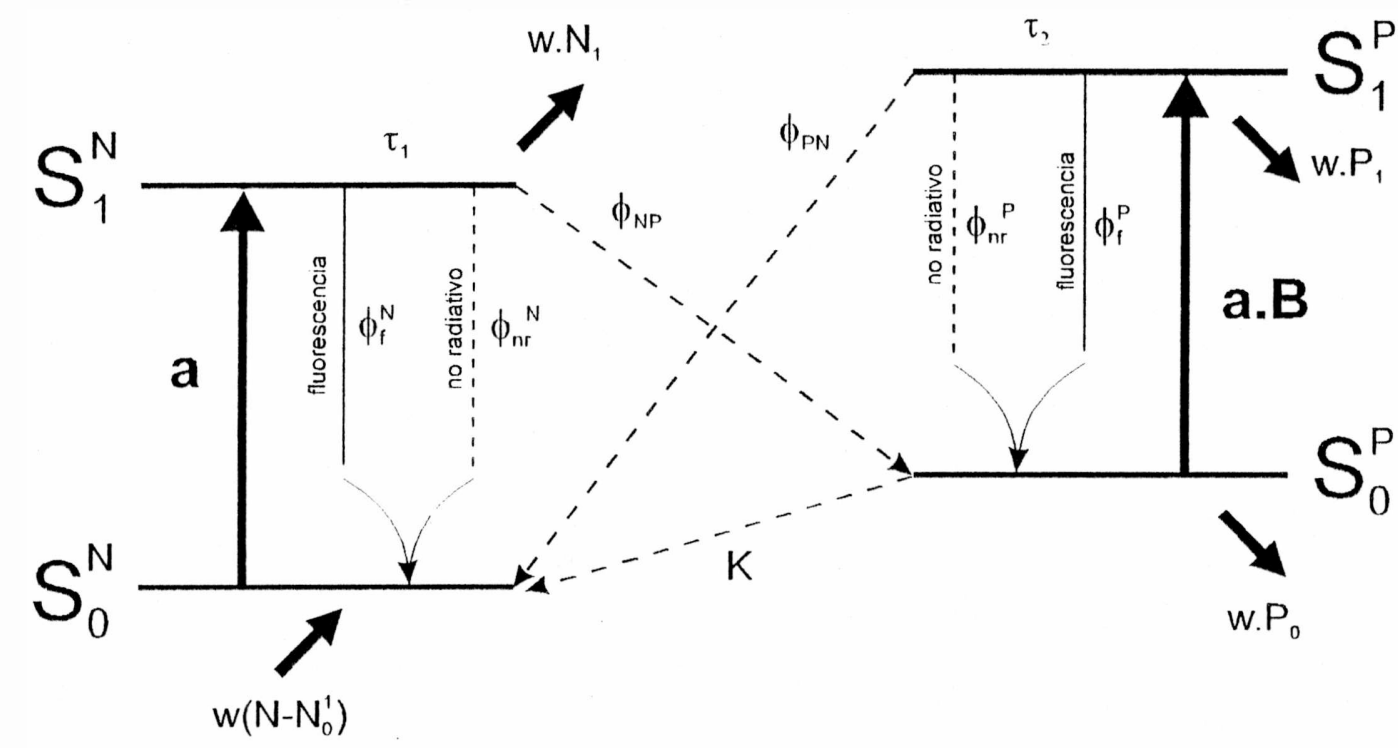

$\mathbf{N}$

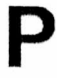

Figura 4.1

Cuando la luz es absorbida por el fotoisómero, la vuelta de $\mathrm{P} \rightarrow \mathrm{N}$ comienza en el nivel $S_{1}^{p}$ y puede terminar en $S_{0}^{N}$ sin pasar por el nivel $S_{0}^{p}$. Este canal de relajación competiría con el mecanismo de vuelta desde el estado fundamental $S_{0}^{\mathrm{p}}$ antes mencionado. Este modelo ha sido aplicado en el tratamiento de la dinámica de fotoisomerización de las diferentes cianinas. El mismo es bastante general e independiente de la estructura estérica real de los isómeros. Si todas las constantes de velocidad involucradas en el proceso son conocidas, el sistema puede ser modelado fácilmente. Con la notación de la figura 4.1, la población de los cuatro niveles involucrados puede ser calculada a partir de las ecuaciones dadas en la siguiente sección. 


\subsubsection{ECUACIONES DE BALANCE DE LAS POBLACIONES}

Resolviendo este sistema de ecuaciones, se puede evaluar la distribución de la población de cada nivel en función de la fluencia de excitación. Esto permitirá ajustar simultáneamente los resultados obtenidos en absorción y fluorescencia al variar dicho parámetro. En condiciones de equilibrio fotoestacionario, la población de todos los niveles involucrados puede ser obtenida en forma exacta. Para excitación pulsada, con diferentes formas de pulso, las poblaciones pueden ser seguidas resolviendo las ecuaciones con métodos numéricos. Si $\mathbf{N}_{\mathbf{0}}, \mathbf{N}_{\mathbf{1}}, \mathbf{P}_{\mathbf{0}}$ y $\mathbf{P}_{1}$ son las poblaciones de los estados fundamental y excitado de las especies normal y fotoisomérica, respectivamente, entonces:

$$
\begin{aligned}
& \frac{d N_{0}}{d t}=-(a+w) \cdot N_{0}+\left(1-\phi_{N P}\right) \cdot \tau_{N}^{-1} \cdot N_{1}+\phi_{P N} \cdot \tau_{P}^{-1} \cdot P_{1}+K \cdot P_{0}+w \cdot N \\
& \frac{d N_{1}}{d t}=a \cdot N_{0}-\left(\tau_{N}^{-1}+w\right) \cdot N_{1} \\
& \frac{d P_{1}}{d t}=B \cdot a \cdot P_{0}-\left(\tau_{P}^{-1}+w\right) \cdot P_{1} \\
& \frac{d P_{0}}{d t}=\phi_{N P} \cdot \tau_{N}^{-1} \cdot N_{1}+\left(1-\phi_{P N}\right) \cdot \tau_{P}^{-1} \cdot P_{1}-(B \cdot a+K+w) \cdot P_{0}
\end{aligned}
$$

$$
\mathbf{N}=\mathbf{N}_{0}+\mathbf{N}_{1}+\mathbf{P}_{0}+\mathbf{P}_{1} \text { se relaciona directamente con la concentración de la }
$$
muestra. $\mathbf{B}=\sigma_{\mathrm{p}} / \sigma_{\mathrm{N}}$, siendo $\sigma_{\mathrm{P}}$ y $\sigma_{\mathrm{N}}$ las secciones transversales de absorción de las especies $\mathrm{P}$ y $\mathrm{N}$ respectivamente. $\mathbf{B}$ depende de la longitud de onda de excitación. $\mathbf{a}=\mathbf{I} . \boldsymbol{\sigma}_{\mathrm{N}}$ $(1 / \mathrm{s})$ es la velocidad de absorción de la luz desde el estado fundamental $\mathrm{S}_{0}^{\mathrm{N}}$, suponiendo una absorbancia baja de tal manera que la intensidad absorbida pueda considerarse proporcional a la primera, donde I ( $\mathrm{fot} /\left(\mathrm{cm}^{2} \mathrm{~s}\right)$ ) es la fluencia de fotones de excitación. w es la velocidad con que se renueva la muestra dentro del volumen irradiado, por circulación de la solución. $K$ es la velocidad del proceso $P_{0} \rightarrow N_{0}$ (desactivación térmica). $\tau_{0}$ y $\tau_{1}$ son los tiempos de vida de los estados excitados de las especies normal y fotoisomérica respectivamente. $\phi_{\mathrm{NP}}$ y $\phi_{\mathrm{PN}}$ son las eficiencias cuánticas de los procesos de isomerización y retroisomerización respectivamente. 
La población térmica del fotoisómero desde el nivel fundamental de la especie normal fue ignorada. Esta aproximación necesita ser justificada en términos de la diferencia de energía entre los estados fundamentales de ambos isómeros (contenido energético del fotoisómero) y es válida para todos los colorantes estudiados en este trabajo. Las ecuaciones completas son desarrolladas por Bäumler y Penzkofer (1990, \#142).

Si en el conjunto de Ec.(4.1) suponemos que la velocidad de desactivación debida a la circulación del flujo (w) es despreciable frente a los demás procesos cinéticos que tienen lugar, el número total de moléculas es aproximadamente igual a:

$$
\mathrm{N} \approx \mathrm{N}_{0}+\mathbf{P}_{0}
$$

donde hemos despreciado la población de los estados excitados respecto de las poblaciones de los estados fundamentales, que en condiciones de equilibrio dependen de los tiempos de vida de cada nivel. La vida media de los estados fundamentales es $10^{4}-10^{6}$ veces mayor que la de los estados excitados.

En estado estacionario, cuando el sistema fotocrómico se halla en equilibrio con la radiación continua de la excitación, las poblaciones de los cuatro niveles involucrados en el modelo pueden ser obtenidas a partir de las ecuaciones ya mencionadas. Despreciando la despoblación de $S_{1}^{N}$ y $S_{1}^{\text {p }}$ por circulación de la solución $\left(w<\tau_{N}^{-1}, \tau_{p}^{-1}\right)$, la fracción de población del estado fundamental de $\mathrm{P}$ puede ser expresada como sigue:

$$
x_{p}=\frac{P_{0}}{N}=\frac{1}{(1+p \cdot B) \cdot\left(1+\frac{a_{s}}{a}\right)}
$$

donde :

$$
\mathbf{p}=\frac{\phi_{\mathrm{PN}}}{\phi_{\mathrm{NP}}} \quad \mathrm{y} \quad \mathbf{a}_{\mathbf{s}}=\frac{\mathbf{K}+\mathbf{w}}{\phi_{\mathrm{NP}} \cdot(\mathbf{1}+\mathbf{p} \cdot \mathrm{B})}
$$

p es el cociente de las eficiencias cuánticas del cruce entre las especies (desde el primer singlete excitado, al estado fundamental de la otra especie). La fluencia de saturación $\left(\mathbf{a}_{\mathrm{s}}\right.$ ) es la necesaria para lograr la mitad de la población máxima que puede alcanzar el fotoisómero. Si la intensidad de excitación es suficientemente alta ( $\mathbf{a}>>\mathbf{a}_{\mathbf{s}}$ ), la ecuación (4.2) se reduce a :

$$
x_{p}=\frac{1}{1+p \cdot B}
$$


Bajo esta condición de excitación, llamada saturación, la población de $S_{0}^{\prime}$ depende de la longitud de onda de excitación, como resultado de la dependencia de $\mathrm{B}$ con este parámetro, y es independiente de la fluencia de excitación. En esta condición el análisis de los resultados se simplifica.

\subsection{TÉCNICAS UTILIZADAS EN LA DETERMINACIÓN DE LOS PARÁMETROS CINÉTICOS Y ESPECTROSCÓPICOS DE LOS COLORANTES.}

La fotofisica de las cianinas ha sido estudiada intensivamente mediante la espectroscopía de absorción y emisión. Como ejemplo se puede mencionar la fotoisomerización del DODCI, analizada usando diferentes fuentes láser con fluencias de excitación muy altas [Mialocq, Shank]. En el caso de colorantes con fotoisómeros que tienen un tiempo de vida relativamente largo en su estado fundamental, como es el caso del DODCI, se pueden usar láseres continuos para producir el fotoequilibrio entre las poblaciones de las especies $\mathrm{N}$ y $\mathrm{P}$. La población $\mathrm{P}_{0}$ obtenida por este método permite estudiar las propiedades ópticas de $\mathrm{P}$ y sus conexiones cinéticas con la fotoisomerización. Los colorantes con especies transientes de vida corta, no pueden ser estudiados con excitación continua y resulta necesario el empleo de láseres pulsados de alta potencia.

\subsubsection{TÉCNICAS DE ABSORCIÓN.}

La espectroscopia láser de absorción resulta ser una técnica muy sensible para el monitoreo de bajas concentraciones de especies conocidas o para caracterizar especies desconocidas. El fotoequilibrio de las formas fotoisoméricas está relacionado con la fluencia de excitación y con los parámetros cinéticos del sistema. Esta relación puede ser analizada a través de medidas de absorción, usando el modelo de isomerización presentado en la sección anterior junto con las ecuaciones que alli aparecen.

Cuando los espectros de absorción de las distintas especies se hallan muy superpuestos, la obtención de información acerca del fotoisómero se vuelve dificultosa. En estos casos se debe emplear una técnica de absorción con doble excitación (pump and probe). Básicamente el método consiste en el empleo de una configuración diferencial, que permite seguir el cambio en la absorbancia de la muestra a través de un haz de prueba incidente, en función de la fluencia del haz de excitación. Como el bombeo es modulado, la 
absorbancia del haz de prueba será diferente dependiendo de si la excitación primaria está presente o no. La reproducibilidad de los resultados experimentales puede mejorarse con detección sincrónica y velocidades altas de muestreo.

El haz modulado de un láser de intensidad $\mathrm{I}_{\mathrm{cxc}}$, incide sobre la muestra generando una población fotoestacionaria $\mathrm{P}_{0}$. La variación de la absorción es monitoreada por un haz de prueba continuo, de intensidad $\mathrm{I} \ll \mathrm{I}_{\mathrm{exc}}$. Utilizando haces polarizados perpendicularmente entre sí, es posible mediante un arreglo adecuado de polarizadores, detectar la señal transmitida de cada haz por separado. Esto es, detectar separadamente el haz de prueba y el de excitación.

Los coeficientes de absorción son obtenidos midiendo la intensidad transmitida del haz de prueba, con y sin excitación (bombeo). Usando la ley de Lambert-Beer para relacionar la intensidad transmitida con la incidente, resulta para el coeficiente de absorción,

$$
\begin{array}{ll}
\alpha_{0}=\frac{1}{l} \cdot \ln \left(\frac{\mathrm{I}}{\mathrm{I}_{0}}\right) & \text { sin excitación } \\
\alpha_{\mathrm{s}}=\frac{1}{l} \cdot \ln \left(\frac{\mathrm{I}}{\mathrm{I}_{\mathrm{s}}}\right) & \text { con excitación }
\end{array}
$$

donde $I$ es la longitud del camino recorrido por el haz dentro de la muestra. I es la intensidad del haz incidente. $\mathbf{I}_{\mathbf{0}} \mathrm{e} \mathbf{I}_{\mathrm{S}}$ son las intensidades transmitidas en cada caso.

Los coeficientes de absorción se relacionan con las secciones eficaces de absorción de los estados fundamentales de N y P. Cuando la excitación está encendida las poblaciones de las dos especies absorben, y sólo la especie normal estará presente cuando no haya excitación. Entonces los coeficientes de absorción pueden ser expresados como sigue:

$$
\begin{aligned}
& \alpha_{0}=N \cdot \sigma_{N} \\
& \alpha_{S}=N_{0} \cdot \sigma_{N}+P_{0} \cdot \sigma_{P}
\end{aligned}
$$

donde $\sigma_{\mathrm{N}}$ y $\sigma_{\mathrm{p}}$ son las secciones eficaces de absorción de las especies $\mathrm{N}$ y $\mathrm{P}$ respectivamente, en la longitud de onda de la prueba. A partir de las últimas ecuaciones podemos escribir la siguiente relación: 


$$
\frac{\alpha_{0}-\alpha_{\mathrm{S}}}{\alpha_{0}}=(\mathbf{B}-1) \cdot \mathbf{x}_{\mathrm{P}}
$$

y utilizando la expresión hallada anteriormente para $\mathbf{x}_{\mathbf{p}}$, resulta:

$$
\frac{\alpha_{0}}{\alpha_{0}-\alpha_{\mathrm{S}}}=\frac{1+\mathbf{p} \cdot \mathbf{B}}{\mathbf{B}-1}+\frac{\mathbf{K}+\mathbf{w}}{(\mathbf{B}-1) \cdot \phi_{\mathrm{NP}}} \cdot \frac{1}{\mathbf{a}}
$$

Esta representa una linea recta cuando es graficada como función de $1 / \mathbf{a}^{\mathrm{H}}$. A partir de esta representación, la excitación en diferentes longitudes de onda permite obtener los valores de $\mathrm{p}, \mathrm{B}$ y $\phi_{\mathrm{NP}}$, siempre que $(\mathrm{K}+\mathrm{w})$, la velocidad de desactivación de $\mathrm{S}_{0}^{\mathrm{P}}$ sea conocida. Como se verá más adelante, este último valor puede determinarse a partir de la modulación resuelta en tiempo de la señal de absorción (figura 4.16).

\subsubsection{TÉCNICAS DE FLUORESCENCIA.}

Las propiedades fluorescentes de las cianinas son particularmente importantes para entender el comportamiento fotofísico del estado singlete excitado de estas moléculas. La emisión fotoisomérica aparece en los casos más favorables, como un cambio en la distribución espectral de la emisión de las especies normales, cuando se varía la fluencia de excitación, o algún otro parámetro como puede ser la temperatura. Pero en el caso general, la presencia del fotoisómero sólo puede ser detectada a través del cambio en la intensidad de la fluorescencia toda (no se observa ningún cambio en la distribución espectral de la fluorescencia). Medidas confiables del espectro de fluorescencia, la eficiencia cuántica de fluorescencia y el tiempo de vida del estado excitado de las especies fotoisoméricas, requieren el uso de técnicas muy sensibles y un análisis cuidadoso del sistema.

En el caso del DODCI [Scaffardi] se ve claramente que el máximo de emisión de su fotoisómero se presenta en una longitud de onda diferente de aquella correspondiente a su especie normal. Para moléculas con espectros de absorción de sus especies que no se hallen fuertemente superpuestos, se puede utilizar una técnica de fluorescencia particularmente apropiada para tratar estos casos. Al igual que la técnica de absorción antes mencionada, esta se basa en la excitación simultánea de la muestra con dos haces, uno continuo y otro

\footnotetext{
* Esta propiedad es particularmente importante para detectar si alguna otra especie interviene en los procesos absorbiendo radiación [Vaveliuk, 1996].
} 
pulsado. El láser continuo, sintonizado en la longitud de onda de absorción de $\mathrm{S}_{0}^{\mathrm{N}}$, produce una población estacionaria $P_{0}$, mientras que el segundo láser (pulsado) prueba esta población en la longitud de onda donde la absorción de $\mathrm{N}$ puede ser despreciada. La fluorescencia transiente viene de $\mathrm{P}$, pudiendo separarse de la emisión de $\mathrm{N}$. Las medidas se realizan con fluencias de bombeo suficientemente altas, tal que las poblaciones en fotoequilibrio de todos los niveles involucrados llegan a ser independientes del valor de excitación (saturación). Medidas de fluorescencia con diferentes fluencias de excitación, permiten obtener el cociente entre la eficiencia cuántica de fluorescencia de la especie fotoisomérica y aquella de la especie normal.

Cuando los espectros de emisión de las especies isoméricas están fuertemente solapados, la determinación de los parámetros cinéticos y espectroscópicos resulta más dificultosa. En estos casos la fluorescencia en cualquier longitud de onda resulta de ambas especies y la intensidad total de fluorescencia debe ser escrita como sigue,

$$
\mathbf{I}_{\mathrm{r}}=\phi_{\mathrm{r}}^{\mathrm{N}} \cdot \mathbf{a} \cdot \mathbf{N}_{0}+\phi_{\mathrm{r}}^{\mathrm{P}} \cdot \mathbf{a} \cdot \mathbf{B} \cdot \mathbf{P}_{0}
$$

Dividiendo ambos miembros por $\phi_{\mathrm{f}}^{\mathrm{N}} \cdot \mathbf{N} \cdot \mathbf{a}$, usando la condición $\mathbf{N}=\mathbf{N}_{\mathbf{0}}+\mathbf{P}_{\mathbf{0}}$ y la expresión hallada para $\mathbf{x}_{\mathrm{p}}$, la ecuación anterior puede volver a escribirse como:

$$
\frac{I_{\mathrm{f}}}{\mathrm{a}}=\mathbf{C} \cdot\left[1+\left(\frac{\phi_{\mathrm{f}}^{\mathrm{P}}}{\phi_{\mathrm{r}}^{\mathrm{N}}} \cdot \mathbf{B}-1\right) \cdot \mathbf{x}_{\mathrm{P}}\right]
$$

y expresando en forma explícita $\mathbf{I}_{\mathbf{f}} / \mathbf{a}$ como función de a :

$$
\frac{I_{f}}{a}=C \cdot \frac{\left(1+G \cdot a / a_{s}\right)}{\left(1+a / a_{s}\right)}
$$

donde $\mathbf{C}$ es una constante instrumental, $\mathbf{G}=(\mathbf{p}+\mathbf{f}) /(\mathbf{p}+\mathbf{1} / \mathbf{B})$ y $\mathbf{f}=\phi_{\mathrm{r}}^{\mathrm{p}} / \phi_{\mathrm{r}}^{\mathrm{N}}$ ( p y $\mathbf{B}$ como fueron definidas antes ).

Cuando la intensidad de fluorescencia dividida la intensidad de excitación es graficada en función de esta última, la ecuación (4.10) puede ser usada para ajustar los datos experimentales. Esta contiene tres parámetros de ajuste: $\mathrm{C}, \mathrm{G}$ y $\mathrm{a}_{\mathrm{s}}$. C depende de factores tales como sensibilidad del fotomultiplicador, longitud de onda, geometría usada en el arreglo experimental, concentración del colorante; y no brinda información útil. $a_{S}$ 
obtenida a partir de las medidas de fluorescencia tiene menos precisión que cuando se obtiene de las medidas de absorción. La información más útil está contenida en el factor $\mathrm{G}$, el valor límite de $\mathrm{I}_{\mathrm{f}} / \mathrm{C}$ a cuando a es mucho mayor que $\mathrm{a}_{\mathrm{s}}$. Teniendo $\mathrm{p}$ y $\mathrm{B}$ de las medidas de absorción, $G$ permite obtener $\mathbf{f}=\phi_{\mathrm{f}}^{\mathrm{P}} / \phi_{\mathrm{l}}^{\mathrm{N}}$, la relación de eficiencias cuánticas de fluorescencia

\subsection{RESULTADOS DE LA APLICACIÓN DE ESTAS TÉCNICAS}

Las técnicas desarrolladas, basadas en estudios de absorción y de fluorescencia en condiciones de equilibrio fotoestacionario, se utilizaron en la determinación de los parámetros fotofísicos de aquellas cianinas fotoisomerizables, que resultaron de interés al propósito de conocer sus conformaciones isoméricas en solución. Se buscó estudiar particularmente, el estado excitado de los fotoisómeros y sus procesos de decaimiento; información ésta que resulta indispensable a la hora de discernir el comportamiento rotacional de estas especies. Técnicas tales como fluorescencia saturada, fluorescencia por doble excitación y absorción en función de la temperatura fueron aplicadas al caso específico del DODCI, permitiendo obtener la eficiencia de fluorescencia y el tiempo de vida de su fotoisómero, en función de la temperatura. Se determinó también la energía de activación y el factor pre-exponecial de los procesos de decaimiento no-radiativo y se registró el espectro de fluorescencia de la forma isomérica transiente, libre de la emisión de la especie normal. Esto constituyó un hecho particularmente importante porque permitió posteriormente estudiar en forma separada del resto, la anisotropía de la fluorescencia de esta especie.

Comenzaré describiendo los resultados de la aplicación de todas estas técnicas al caso del DODCI y luego continuaré con los resultados obtenidos para las tres restantes cianinas estudiadas, en las que el fuerte solapamiento que presentan las secciones eficaces de absorción y fluorescencia de ambas especies isoméricas dificulta analizarlas separadamente.

\subsubsection{DODCI}

Las medidas se llevaron a cabo utilizando colorante de grado láser, Eastman Kodak, en solución de etanol absoluto, de grado analítico. Para la detección en los cambios de la 
absorbancia y detección de la emisión fluorescente, se ha empleado una solución del colorante en etanol de baja concentración $\left(2-10 \times 10^{-6} \mathrm{M}\right)$, para evitar efectos de reabsorción y formación de agregados, asegurando una concentración homogénea de todas las especies dentro de la celda. Mediante la acción de una pequeña bomba la solución se hizo circular a velocidad constante, desde un reservorio ubicado en un baño termostatizado hasta la celda de cuarzo ( una cubeta standard de fluorescencia, con forma de paralelepípedo y sección transversal cuadrada, de dimensiones $1 \times 1 \times 4 \mathrm{~cm}$ ). La celda se ubicó en un portacelda adecuado, con ventanas que permitieron al mismo tiempo la excitación del compuesto, la observación de los cambios en la absorción y la detección de la fluorescencia. La temperatura fue medida en la celda, con un error de $\pm 0.2^{\circ} \mathrm{C}$, mediante el empleo de una termocupla de $\mathrm{Cu}$-constantan. En el rango de temperaturas estudiado, de 0 a $55^{\circ} \mathrm{C}$, se supuso una dependencia de la viscosidad con la temperatura de tipo Arrhenius. (En la última página del apéndice $\mathrm{C}$, se indican los parámetros usados). La velocidad con que se removió la muestra de la zona iluminada por circulación de la solución, w, está relacionada con la velocidad del flujo en la celda, $v$, y el diámetro de la zona irradiada, d. Después de medir la velocidad del flujo, $w=v / d$ fue estimada en $\sim 10^{3} 1 / \mathrm{s}$.

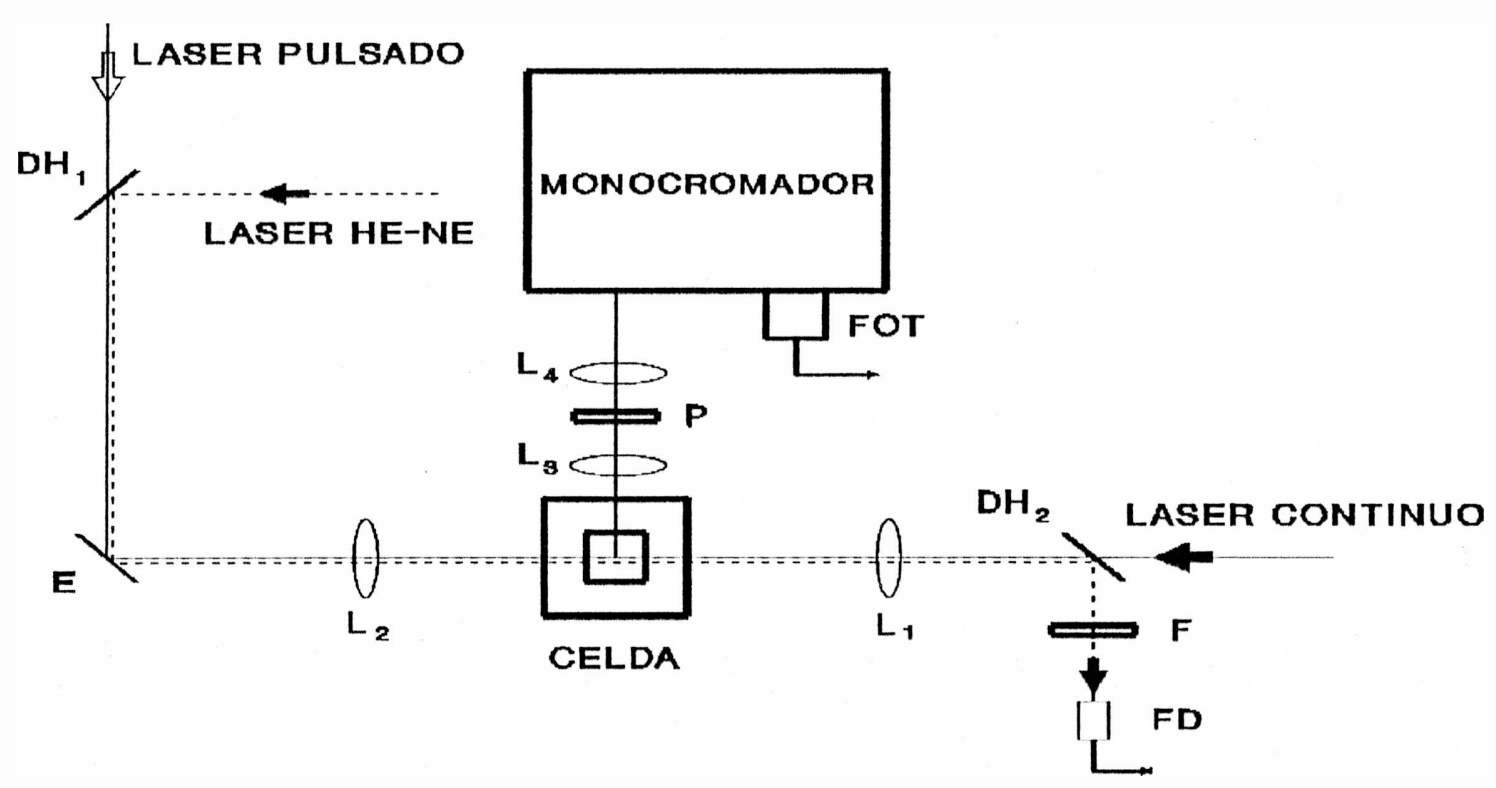

Figura 4.2

Las medidas de absorción y fluorescencia se realizaron utilizando el esquema experimental mostrado en la figura (4.2). Para la fluorescencia, se enfocó sobre la celda un 
láser de colorante modelo Spectra Physics 375 (Rodamina 6G) bombeado por un láser de argón o el mismo láser de Ar modelo Spectra Physics SP 165. Simultáneamente, un láser de colorante bombeado por una lámpara de flash (Chromatix CMX-4, ancho del pulso $=1 \mu \mathrm{s}$, energía pico $=1 \mathrm{~mJ}$, velocidad de repetición $=5 \mathrm{~Hz}$ ) se alineó en sentido opuesto con respecto al láser continuo y se focalizó con la lente L2, sobre la misma región espacial de la celda que iluminara el primer haz. La fluorescencia pulsada fue detectada perpendicularmente a la dirección de excitación. La polarización de los dos haces láser, pulsado y continuo, era perpendicular al plano definido por las direcciones de incidencia de los haces de excitación y de observación de la fluorescencia. La fluorescencia fue focalizada a través de un sistema de lentes, $\mathrm{L}_{3}$ y $\mathrm{L}_{4}$, sobre la rendija de un espectrómetro de barrido (Jarrell Ash 82-025), con montaje Ebert y $50 \mathrm{~cm}$ de distancia focal. Entre las lentes se colocó un polarizador orientado en ángulo "mágico", para eliminar los efectos sobre la fluorescencia de la difusión rotacional, efectos que pueden ser distintos para las diferentes especies de un mismo compuesto. La respuesta del sistema monocromadorfotomultiplicador difiere significativamente para polarización paralela o perpendicular a la ranura del monocromador. El sistema fue calibrado usando una lámpara patrón de tungsteno. El espectro de la lámpara (a) y las señales registradas por el sistema para polarización paralela (b) y perpendicular (c) a la ranura, son mostrados en la figura (4.3). Para poder corregir los espectros un segundo polarizador se colocó delante del monocromador, con su linea de transmisión vertical debido a la dependencia más suave del sistema de detección para la polarización paralela a la ranura, (Fig. 4.3b). La emisión se detectó usando un fotomultiplicador multialcalino Hamamatsu R-446, adosado a la salida del espectrómetro.

Para las medidas de absorción, en lugar del láser de colorante pulsado, se usó un láser continuo de He-Ne de baja potencia (línea a trazos en la Fig. 4.2). Con un fotodiodo (Spectra Physics, modelo 404) se midió la potencia del láser de He-Ne transmitida a través de la celda con $\left(\mathrm{I}_{\mathrm{s}}\right)$ y $\sin \left(\mathrm{I}_{\mathrm{o}}\right)$ excitación del haz de bombeo. Por las características de absorción de una y otra especie del DODCI, el coeficiente de absorción calculado como $\alpha_{\mathrm{P}^{\mathrm{p}}}=\ln \left(\mathbf{I}_{\mathrm{S}} / \mathbf{I}_{0}\right)$ se relaciona con $\mathbf{P}_{\mathbf{0}}$ por la siguiente igualdad:

$$
\alpha_{\mathrm{P}}=\sigma_{\mathrm{P}} \cdot \mathbf{P}_{0} \cdot \boldsymbol{l}
$$

donde $l$ es la longitud de la celda y $\sigma_{\mathrm{r}}$, es la sección transversal de absorción del fotoisómero en $\lambda=632.8 \mathrm{~nm}$. La intensidad absorbida por la especie normal es despreciable en esta longitud de onda. Las señales de absorción y de fluorescencia se procesaron mediante el 
empleo de un integrador Boxcar (EG \& G PAR 162-163) y fueron presentadas en un gráfico Y-t.

\section{Curva de Calibración}

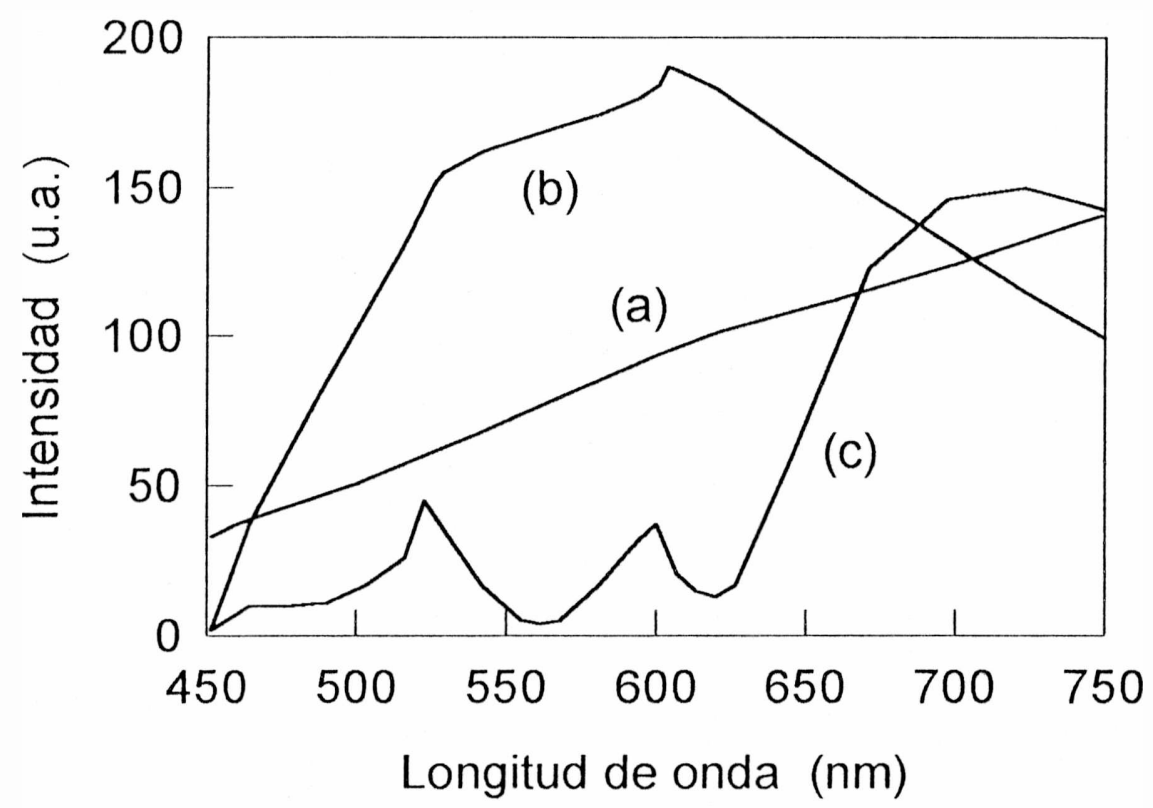

Figura 4. 3. Calibración del sistema de detección. (a) Espectro de la lámpara patrón, (b) respuesta del sistema para polarización paralela y (c) respuesta para polarización perpendicular.

En lo que sigue se muestran los distintos resultados obtenidos en el estudio de este colorante.

- DETERMINACIÓN DE LA POBLACIÓN P ${ }_{0}$ EN FUNCIÓN DE LA TEMPERATURA

Excitando con el láser de colorante en forma continua y con alta fluencia en $570 \mathrm{~nm}$, se logró poblar significativamente el estado fundamental del fotoisómero a partir de la especie normal y del pasaje $\mathrm{S}_{1}^{\mathrm{N}} \rightarrow \mathrm{S}_{0}^{\mathrm{P}}$. La población transferida a $\mathrm{S}_{0}^{\mathrm{p}}$ es seguida por el cambio en la transmisión del láser de He-Ne, que prueba esta población en su zona de absorción (ver Fig. 5.7). El haz del primer láser fue modulado para obtener la diferencia entre las 
intensidades transmitidas con y sin población inducida. La ligura 4.4 muestra la población del fotoisómero, nomalizada con su valor a temperatura ambiente, para distintas temperaturas entre 0 y $55^{\circ} \mathrm{C}$. En la determinación de esta población se usó la Eq. (4.11).

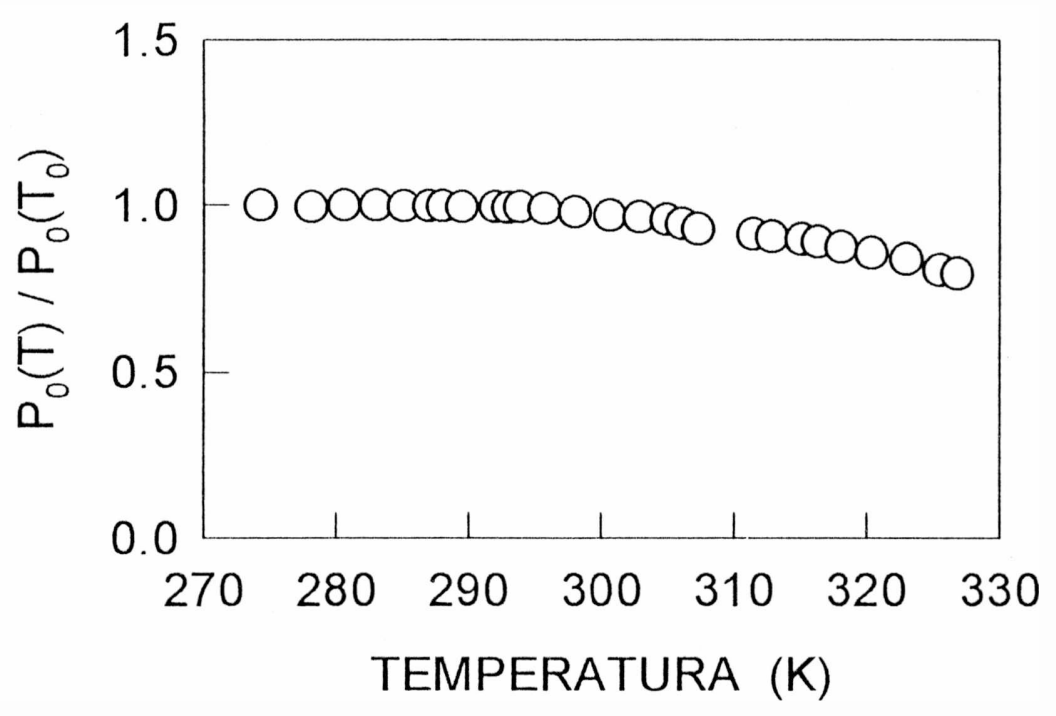

Figura 4.4. Variación de la población del fotoisómero con la temperatura.

\section{- población Po en régimen estacionario, bajo condición de SATURACIÓN.}

La saturación puede ser definida como la condición para la cual la absorción de P es independiente de la fluencia de bombeo. Bajo esta condición, el coeficiente de absorción de esta especie $\left(\alpha_{1}\right)$ se midió en distintas longitudes de onda de excitación con el fin de obtener la dependencia de $P_{0}$ con este parámetro. En la figura 4 .5a se muestran los valores de $\alpha_{1}$, normalizados al valor obtenido con el bombeo en el punto isosbéstico $(B=1)$. Para DODCI este punto corresponde a $\lambda_{13-1}=600 \mathrm{~nm}$.

Los valores experimentales pueden ser ajustados con una expresión que resulta de combinar las ecuaciones (4.3) y (4.11).

$$
\frac{\alpha_{\mathrm{P}}(\lambda)}{\alpha_{\mathrm{P}}\left(\lambda_{0}\right)}=\frac{\mathrm{P}_{0}(\lambda)}{\mathrm{P}_{0}\left(\lambda_{0}\right)}=\frac{1+\mathrm{p}}{1+\mathrm{p} \cdot \mathrm{B}}
$$


El mejor ajuste se obtuvo con $\mathbf{p}=0.36 \pm 0.06$. B fue calculado a partir de los valores de $\sigma$ obtenidos del trabajo de Bilmes (1988). El buen ajuste de todos los datos respalda la independencia de p con la longitud de onda de bombeo. Usando la Eq.(4.3), con el valor de p hallado, podemos obtener la población $\mathrm{P}_{0}$. En la figura $4.5 \mathrm{~b}$ puede verse cómo en la condición de saturación casi todas las moléculas son convertidas de $\mathrm{N}$ a $\mathrm{P}$ para $\lambda<580 \mathrm{~nm}$ $(\mathrm{B}<1)$, mientras que para $\lambda>600 \mathrm{~nm}(\mathrm{~B}>1$ ) la retroisomerización es un canal efectivo para volver de $\mathrm{P}$ a $\mathrm{N}$.

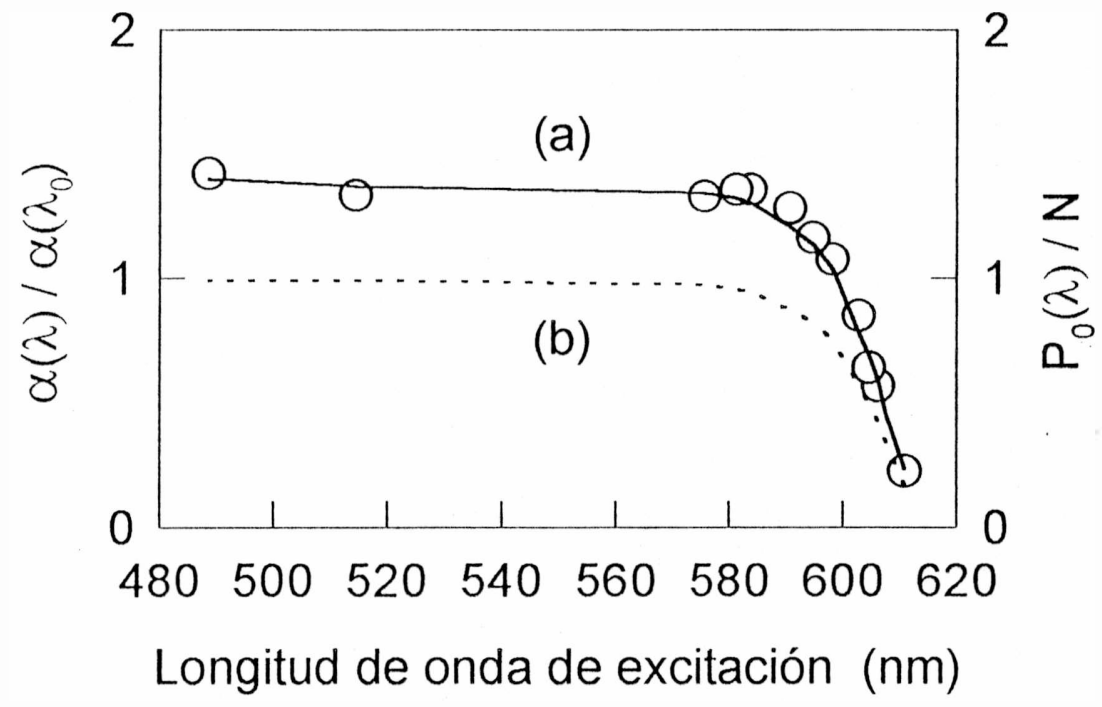

Figura 4.5. Los circulos corresponden a valores relativos del coeficiente de absorción del fotoisómero. In línea continua (a) se muestra el ajuste obtenido usando la Eq. (4. 12). La línea a trazos (b) representa la fracción de población del fotoisómero en su estado fundamental.

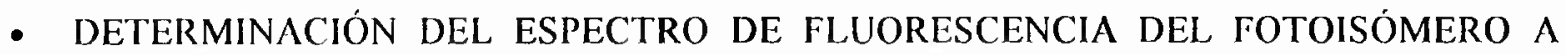
TEMPERATURA AMBIENTE.

La excitación en forma continua de la muestra con alta fluencia $\left(\mathrm{I}_{0}=300 \mathrm{~W} / \mathrm{cm}^{2}\right.$ ) en $580 \mathrm{~nm}$, transfiere aproximadamente todas las moléculas a $P_{0}$. Dado que la absorción del fotoisómero en esta longitud de onda es pequeña, el espectro de emisión fluorescente obtenido sólo corresponde a la especie normal. Excitando luego en forma pulsada en 640 $n m$, al ser $\sigma_{p}, P_{0} \gg \sigma_{N}, N_{n}$ para esta longitud de onda, se logra poblar $S_{1}^{p}$, resultando posible obtener el espectro de fluorescencia del foloisómero, detectando la señal pulsada. 
La figura 4.6 muestra la señal fluorescente pulsada que se obtiene con esta técnica de doble excitación. El espectro más pequeño (4.6b) corresponde a la señal obtenida sin la excitación del láser continuo. La fluorescencia desde $S_{1}^{N}$ que aparece cuando el bombeo está encendido, genera una señal continua que es filtrada por la baja impedancia del sistema de detección ( $50 \Omega$ ). La flecha en la figura indica la posición del máximo de emisión de la especie normal. El pico producido por scattering del haz de prueba pulsado puede ser obtenido usando solvente puro en la celda y luego restado con el fin de corregir el espectro.

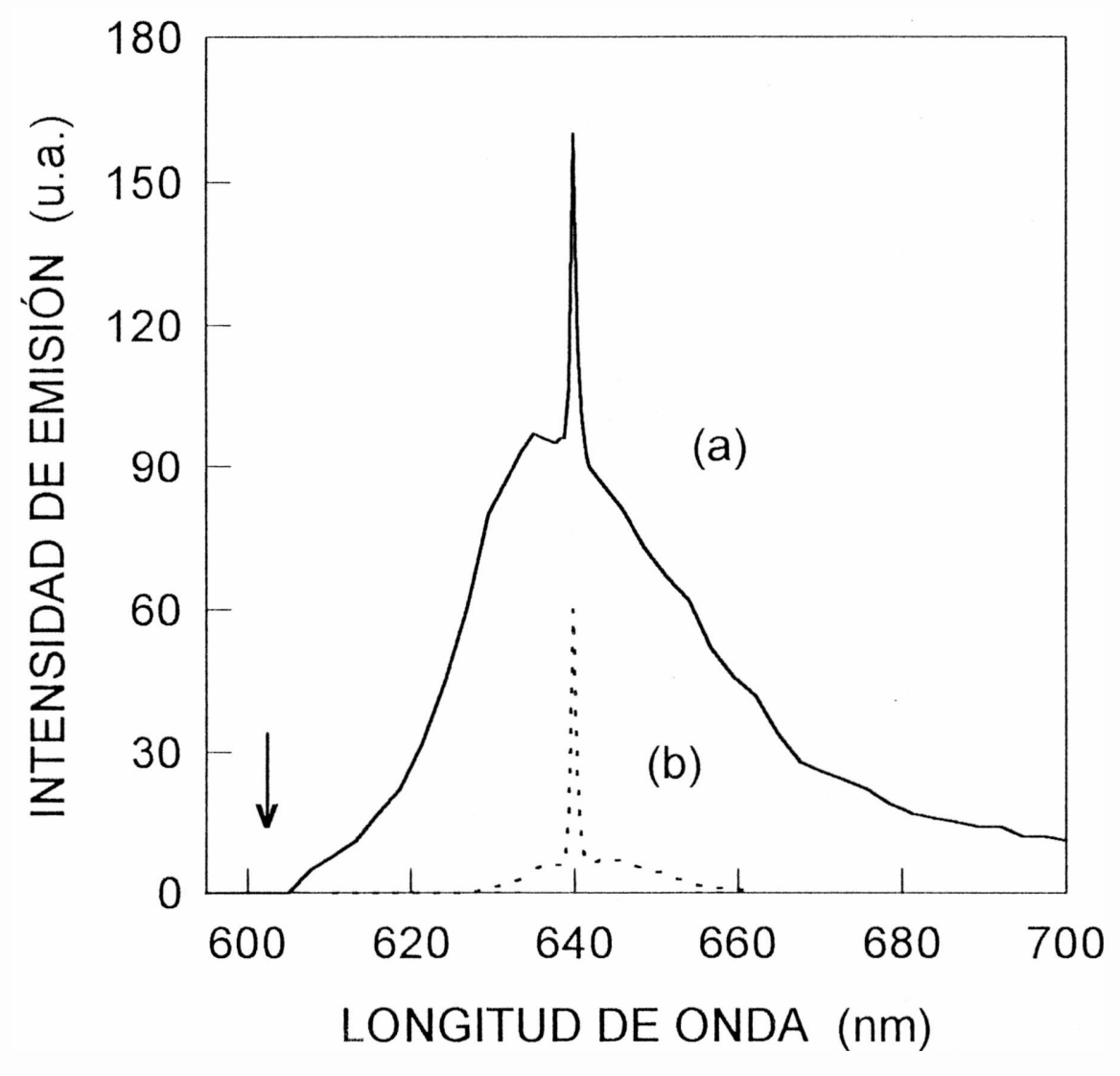

Figura 4.6. Espectro de fluorescencia del fotoisómero del DODCI.

(a) Espectro obtenido usando la técnica de doble excitación.

(b) Espectro de emisión que resulta de usar sólo la excitación pulsada.

Para obtener la eficiencia cuántica de fluorescencia de la forma fotoisomérica del DODCI, la emisión de esta especie fue comparada con la emisión de la especie normal del DTDCI, seleccionado por sus similares propiedades de absorción-emisión. La muestra con 
DODCI fue cambiada por una conteniendo el nuevo colorante, se quitó la excitación continua, y se usó la misma excitación pulsada de baja energía. El cociente de las emisiones que se obtuvieron (áreas bajo las curvas de fluorescencia) fue 0.254. Usando para DTDCI, $\phi_{\mathrm{r}}^{N}=0.36 \pm 0.05$ [Bilmes, 1989], el valor absoluto para la eficiencia cuántica de fluorescencia de $\mathrm{P}$ se calculó en $\phi_{\mathrm{r}}^{\mathrm{p}}(\mathrm{DODCI})=0.09 \pm 0.02$. Si bien $\phi_{\mathrm{r}}^{\mathrm{P}}$ resulta ser más alto que el valor $0.06 \pm 0.02$ dado por Bilmes (1988) para esta misma especie, estos dos valores son compatibles dentro del error experimental. Con el espectro de absorción del isómero $\mathrm{P}$ tomado de la última referencia y usando la fórmula de Strickler y Berg, el tiempo de vida radiativo de esta especie fue calculado en, $\tau_{0}=4.0 \mathrm{~ns}$.

\section{- ANÁLISIS DE LA CONVERSIÓN DIRECTA $S_{1}^{p} \rightarrow S_{1}^{N}$.}

La posibilidad de la conversión directa $S_{1}^{1} \rightarrow S_{1}^{N}$ [Arthurs (1973), Bäumler (1990, \#140)] puede ser discutida usando los espectros de emisión mostrados en la figura 4.6. La población $N_{1}$ alcanzada puede ser analizada en términos de los siguientes procesos: absorción directa por $S_{0}^{N}$ o absorción por $S_{0}^{p}$ seguida por el pasaje desde $S_{1}^{p}$ a $S_{1}^{N}$. La velocidad de transferencia de población en el último caso resulta en un máximo. Este liecho es debido a la baja población de $S_{0}^{N}$ y al pequeño coeficiente de absorción de la especie normal en la longitud de onda correspondiente a la excitación pulsada. Vale decir entonces que $S_{1}^{N}$ sólo es poblado a través del pasaje $S_{1}^{p} \rightarrow S_{1}^{N}$. En este caso,

$$
\frac{\mathrm{dN}_{1}}{\mathrm{dt}}=\mathrm{k}_{\mathrm{PN}}^{1} \cdot \mathrm{P}_{1}-\frac{\mathrm{N}_{1}}{\tau_{\mathrm{N}}} \cong 0 \quad \rightarrow \quad \mathrm{N}_{1}=\mathrm{k}_{\mathrm{PN}}^{1} \cdot \tau_{\mathrm{N}} \cdot \mathrm{P}_{1}
$$

donde las condiciones estacionarias para $\mathrm{N}_{1}$ y $\mathrm{P}_{1}$ son válidas, dado que la duración del pulso es mucho mayor que los tiempos de vida de los estados excitados. El cociente de las emisiones fluorescentes provenientes de $S_{1}^{N}$ y $S_{1}^{P}$ es estimado de,

$$
\frac{\mathrm{I}\left(\lambda_{\mathrm{N}}\right)}{\mathrm{I}\left(\lambda_{\mathrm{P}}\right)}=\frac{\frac{\mathrm{N}_{1} \cdot \mathrm{E}\left(\lambda_{\mathrm{N}}\right)}{\tau_{\mathrm{N}} \cdot \lambda_{\mathrm{N}}}}{\frac{\mathrm{P}_{1} \cdot \mathrm{E}\left(\lambda_{\mathrm{P}}\right)}{\tau_{\mathrm{p}} \cdot \lambda_{\mathrm{p}}}} \cong \phi_{\mathrm{PN}}^{1} \cdot \frac{\phi_{\mathrm{f}}^{\mathrm{N}}}{\phi_{\mathrm{f}}^{\mathrm{P}}} \rightarrow \phi_{\mathrm{PN}}^{1} \cong \frac{\mathrm{I}\left(\lambda_{\mathrm{N}}\right)}{\mathrm{I}\left(\lambda_{\mathrm{P}}\right)} \cdot \frac{\phi_{\mathrm{f}}^{\mathrm{P}}}{\phi_{\mathrm{f}}^{\mathrm{N}}}
$$

donde $\lambda_{\mathrm{N}}$ y $\lambda_{\mathrm{p}}$ corresponden a los máximos de emisión de cada especie y $E(\lambda)$ es la forma de linea de los espectros de emisión, los cuales se asumen iguales para las dos especies. Tomando el cociente de intensidades como 0.01 y el cociente de eficiencias como 0.225 
$\left(\phi_{\mathrm{r}}^{\mathrm{N}}=0.4\right.$ [Rulliere] y $\phi_{\mathrm{r}}^{\mathrm{P}}=0.09$, de la sección anterior $)$, resulta $\phi_{\mathrm{PN}}^{1} \leq 2.2510^{-3}$, un valor que puede ser despreciado en relación a $\phi_{\mathrm{P}^{\mathrm{N}}}$

El proceso directo es más dificil de estimar. Una sobreestimación límite puede ser obtenida suponiendo un modelo de dos valles para la superficie de energía potencial $S_{1}$, desestimando la existencia del estado twisted. En este caso,

$$
\mathbf{k}_{\mathrm{NP}}^{1}=\mathbf{k}_{\mathrm{PN}}^{1} \cdot \exp (\Delta \mathbf{E} / \mathbf{k T})
$$

donde $\Delta \mathbf{E}=\mathbf{E}_{\mathrm{N}}^{1}-\mathbf{E}_{\mathrm{P}}^{1}$ es la diferencia de energía entre los niveles $\mathrm{S}_{1}^{\mathrm{N}}$ y $\mathrm{S}_{1}^{\mathrm{P}}$. El valor más alto de $\mathbf{k}_{\mathrm{NP}}^{\prime}$ se puede obtener usando un valor de energía de $\Delta \mathbf{E}=770 \mathrm{cal} / \mathrm{mol}$, correspondiente a la diferencia de energía más alta reportada entre aquellos niveles [Bäumler (1990, \#140)]. A temperatura ambiente resulta, $\mathbf{k}_{\mathrm{NP}^{\mathrm{P}}}^{1} \leq 910^{-3} \mathrm{~ns}^{-1}$ y $\phi_{\mathrm{N}^{2}}^{1} \leq 210^{-2}$. Considerando también el decaimiento a través del estado twisted, estos valores deberian ser mucho más bajos. Entonces, en una primera aproximación, el cruce $S_{1}^{P} \leftrightarrow S_{1}^{N}$ puede ser desestimado.

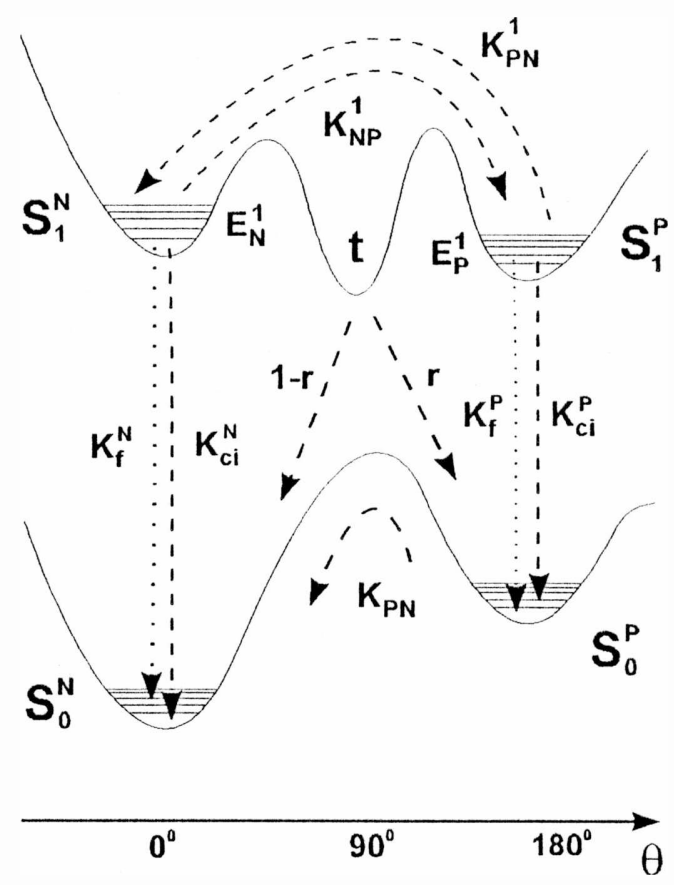

Diagrama de energia potencial considerando un modelo de tres valles para la superficie $S_{1}$. Lista figura es complementaria de la figura 2.4. Lit factor r que aparece en el dibujo da cuenta de como se reparten las moléculas desde el estado twisted hacia el estado findamental de ambas especies isoméricas. En relación con la figura 2.4, $r$ se vincula con las velocidades de desactivación de la sigulente manera: $K_{N P}=r \cdot K_{N t}, K_{P N}=(1-r) \cdot K_{P t}$ 
- dependencia con la temperatura de la eficiencia cuántica de FLUORESCENCIA Y DEL TIEMPO DE VIDA DEL FOTOISÓMERO

Cuando una población de equilibrio $\mathrm{P}_{0}$ es alcanzada por el bombeo continuo a una dada temperatura, la amplitud de la señal fluorescente pulsada, I(T), puede ser considerada proporcional a la población $P_{0}$ y a la eficiencia de fluorescencia $\left(\phi_{r}^{P}\right)$ a esa temperatura. $P_{0}$ fue medida como se describió anteriormente, entre 0 y $55^{\circ} \mathrm{C}$. En la figura 4.4 se mostraron los valores de $P_{0}$ relativos a su valor a temperatura ambiente. La intensidad de la emisión nuorescente del fotoisómero, producida por la prueba pulsada en $640 \mathrm{~nm}$, se midió en el mismo rango de temperaturas. Esto permitió determinar la dependencia de la eficiencia cuántica de fluorescencia con este parámetro. $\phi_{\mathrm{f}}^{\mathrm{p}}$ fue calculada para cada temperatura como,

$$
\phi_{r}^{\mathrm{P}}(\mathrm{T})=\frac{I(T)}{I\left(\mathrm{~T}_{0}\right)} \cdot \frac{\mathbf{P}_{0}\left(\mathrm{~T}_{0}\right)}{\mathbf{P}_{0}(\mathrm{~T})} \cdot \phi_{\mathrm{r}}^{\mathrm{P}}\left(\mathrm{T}_{0}\right)
$$

Los valores obtenidos se muestran en la siguiente figura.

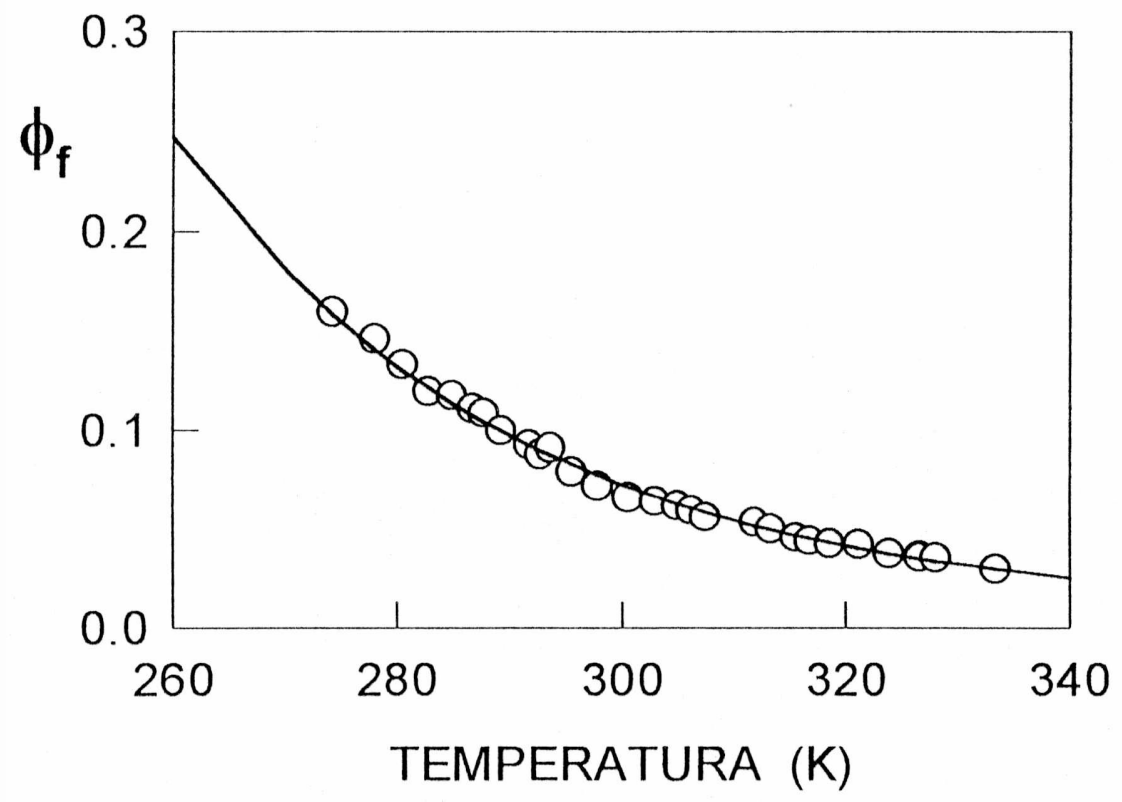

Figura 4.7 
Usando $\phi_{\mathrm{r}}^{\mathrm{P}}(\mathrm{T})$ y el tiempo de vida radiativo del fotoisómero que se halló antes $\left(\tau_{0}=4.0 \mathrm{~ns}\right)$, el tiempo de vida de $S_{1}^{\text {? }}$ para cada temperatura, puede ser calculado como,

$$
\tau_{\mathrm{P}}(\mathrm{T})=\tau_{0}^{\mathrm{P}} \cdot \phi_{\mathrm{r}}^{\mathrm{P}}(\mathrm{T})
$$

A partir de $\tau_{0}^{p}$ y $\tau_{\mathbf{p}}$, la dependencia con la temperatura del proceso no radiativo, puede ser analizada como sigue,

$$
\mathbf{k}_{\mathrm{nr}}^{\mathrm{P}}=1 / \tau_{\mathrm{P}}-1 / \tau_{0}^{\mathrm{P}}
$$

Los resultados se presentan de la manera usual (plot de Arrhenius) en la figura 4.8. La linealidad del plot indicaría que sólo un proceso no-radiativo está involucrado en este rango de temperaturas. La energía de activación para este proceso se evaluó en $\mathbf{E}_{\mathrm{nr}}^{\mathrm{p}}=5740$ $\pm 100 \mathrm{cal} \mathrm{mol}^{-1}$. Este valor resulta similar a aquel reportado para las energías de activación de los procesos no-radiativos desde $\mathrm{S}_{1}^{\mathrm{N}}$, la isomerización $\left(\mathrm{k}_{\mathrm{NP}}\right)$ [Velsko, 1982] y la retroisomerización $\left(\mathrm{k}_{\mathrm{PN}}\right)$ [Duchowicz, 1990].

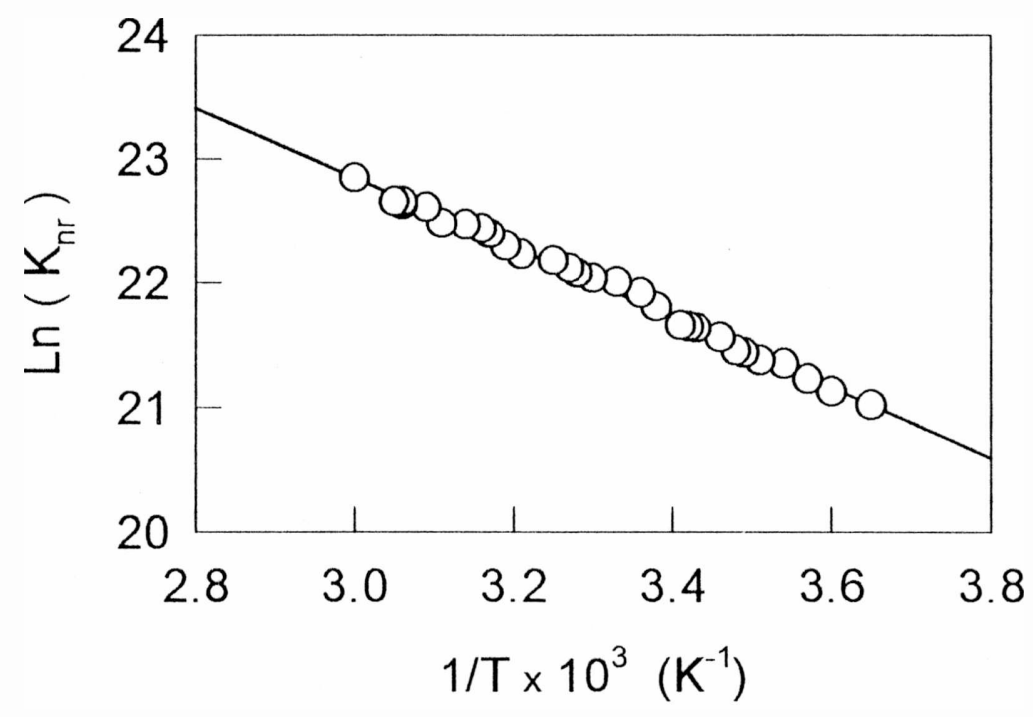

Figura 4.8

No se hallaron contribuciones del proceso no-radiativo independientes de la temperatura. De no existir una desactivación tal, la velocidad del proceso no-radiativo puede ser escrita como, 


$$
\mathbf{k}_{\mathrm{nr}}^{\mathrm{p}}=\mathbf{F}(\boldsymbol{\eta}) \cdot \exp \left(-\mathbf{E}_{0}^{\mathrm{P}} / \mathbf{R T}\right)
$$

donde $\mathbf{F}(\eta)$ es una función universal de la viscosidal y $\mathbf{E}_{0}^{\prime \prime}$ es la altura de la barrera molecular intrínseca [Velsko, 1982], la cual está relacionada sólo con las propiedades moleculares. Usando diferentes solventes (metanol, metanol-agua, etanol y propanol) se construyó un plot de Arrhenius de viscosidad constante, para obtener $\mathbf{E}_{0}^{\mathrm{P}}$. Para que la viscosidad de los cuatro solventes sea la misma, se midió a una temperatura diferente en cada caso. Los resultados para $\eta=0.9 \mathrm{cP}$, se muestran en la figura 4.9. Se obtuvo una barrera intrínseca $\mathbf{E}_{0}^{\mathrm{P}}=2830 \pm 200 \mathrm{cal} \mathrm{mol}^{-1}$. Este resultado es similar a aquel obtenido para el decaimiento no-radiativo de $S_{1}^{N}$ [Velsko, 1982].

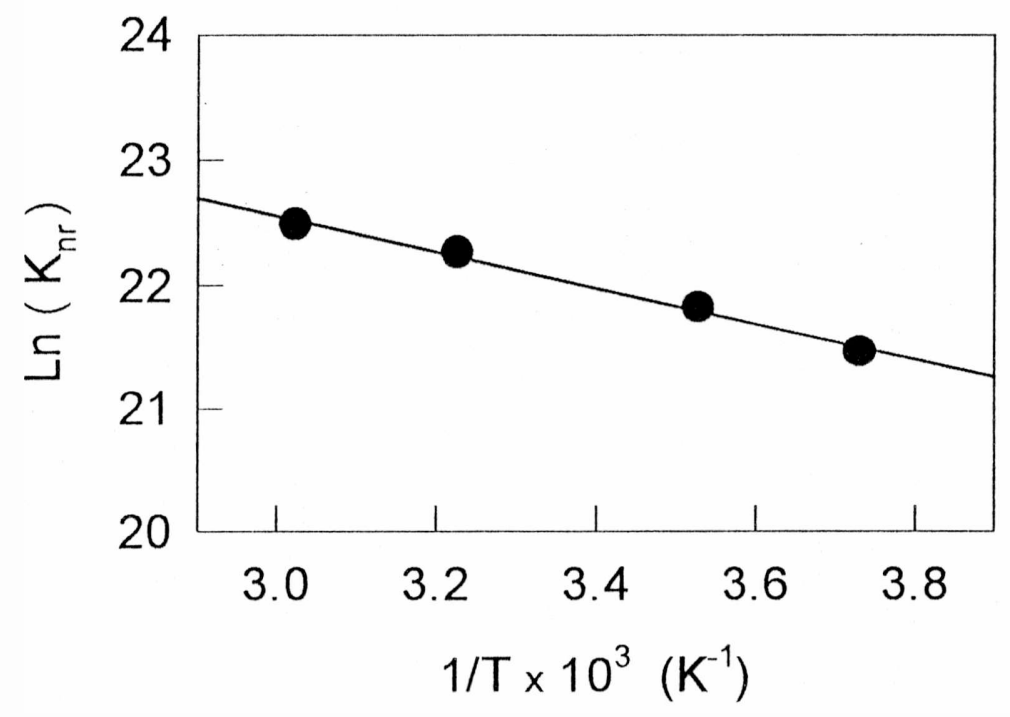

Figura 4.9

En resumen, como resultado de la aplicación de las técnicas mencionadas, se logró obtener información de la especie transiente del DODCI, minimizando la influencia de su forma normal. De esta manera se obtuvo el espectro fluorescente, la eficiencia de fluorescencia, el tiempo de vida y la población de su estado fundamental, en función de la temperatura. Excitando en forma continua con alta fluencia fue posible obtener datos de la barrera de potencial que influencia el proceso de retroisomerización $\left(\mathrm{k}_{\mathrm{pN}}\right)$. A partir de las medidas de absorción, con alta fluencia de excitación, se pudo obtener la población $\mathrm{P}_{0}$ en función de la longitud de onda de excitación y comprobar el modelo fotoisomérico 


\subsubsection{DTCI}

Al igual que con el DODCI, la solución de DTCI (Exciton) se hizo fluir a través de una cubeta de fluorescencia standard. Se trabajó con soluciones de etanol grado analitico a temperatura ambiente $\left(20^{\circ} \mathrm{C}\right)$. Para evitar efectos de dimerización se usaron soluciones de baja concentración. En la figura 4.10 se muestra el arreglo experimental que se usó para las medidas de absorción láser y para las de fluorescencia inducida por láser, tal como se describieron previamente (sección 4.4).

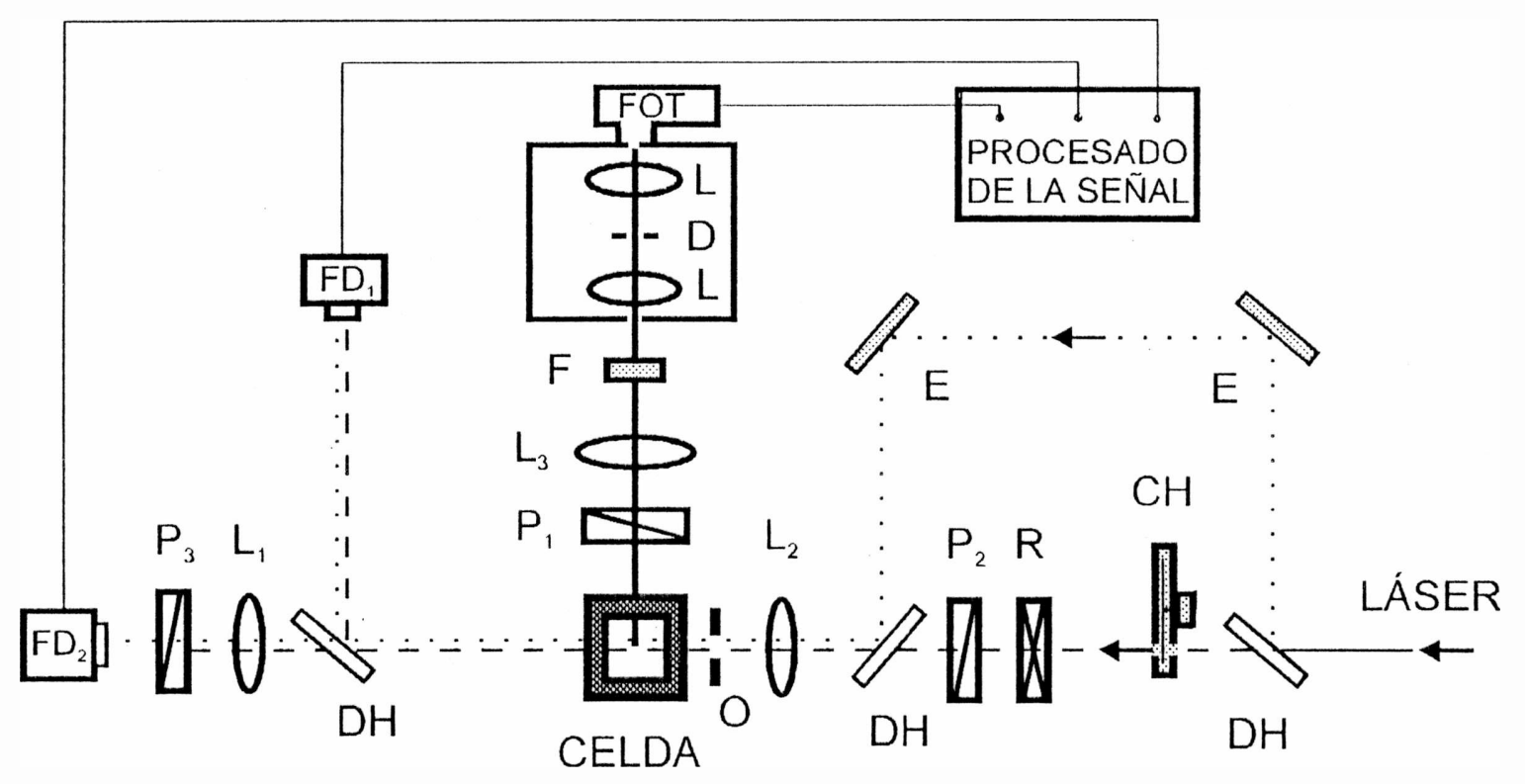

Figura 4. 10. Esquema experimental. La linea de puntos representa al haz de prueba, la linea a trazos al de excitación. La polarización del primer haz es perpendicular al plano del arreglo experimental. El haz de excitación tiene polarización paralela a aquel plano después de atravesar el polarizador $P_{2}$. Los distintos componentes del arreglo son: DH divisor de haz; $R$ lámina de media onda; (H chopper; P polarizador; li espejo; O orificio calibrado; L. lente; I filtro; D diafragma; FD fotodiodo; FOT fotomultiplicador.

El haz de prueba y el de bombeo los provee el mismo láser. Para ello el haz láser es dividido en dos haces mediante el arreglo de espejos y divisores de haz esquematizado en la figura. Uno de prueba indicado con línea punteada y otro de excitación en línea de trazos Los dos haces fueron enfocados sobre la celda con una lente plano-convexa $(f=7 \mathrm{~cm})$. El uso adecuado de los polarizadores $\left(P_{2}\right.$ y $\left.P_{3}\right)$, permite detectar separadamente la señal de prueba y la intensidad de excitación. El fotodiodo $\mathrm{FD}_{2}$ detecta sólo la intensidad del haz de prueba, $I_{0}$. El polarizador $P_{3}$ colocado delante de $F_{2}$, con su eje de transmisión en la 
dirección de polarización del haz de prueba, impide el paso del haz de excitación polarizado perpendicularmente respecto del primero por acción de $\mathrm{P}_{2}$. El fotodiodo $\mathrm{FD}_{1}$ detecta la intensidad total de la excitación láser que atraviesa la muestra $\left(I_{0}+I_{e}\right.$, siendo $I_{c}$ la intensidad del haz de excitación). Para amplificar las señales detectadas por los fotodiodos se utilizaron amplificadores de continua. Un osciloscopio digital permitió visualizar estas señales

El arreglo experimental esquematizado en la figura 4.10, permite variar la intensidad $I_{c}$ del haz de excitación rotando la lámina de media onda, y de esta forma modular la población estacionaria del estado fundamental del fotoisómero. La variación de la absorción resultante es monitoreada por el haz de prueba, de longitud de onda $\lambda_{0}$ (notar que ahora, a diferencia del estudio realizado con DODCI, $\lambda_{0}=\lambda_{\mathrm{e}}$ ), intensidad incidente constante $\mathrm{I}_{0}$ $\left(I_{0} \ll I_{c}\right)$ y polarización rotada en $\pi / 2$ respecto del haz de excitación

La excitación continua con el láser se hizo en cinco longitudes de onda diferentes; con el láser de argón en 501.7 y 514.5 nm, y con el láser de colorante (Rodamina 6G) bombeado por el láser de argón, en 562,557 y $550 \mathrm{~nm}$.

\section{RESULTADOS DE LAS MEDIDAS DE ABSORCIÓN}

La espectroscopía de absorción se utilizó para analizar el fotoequilibrio entre ambos isómeros de esta molécula, en términos de la fluencia de excitación. Una vez más se estudió el cambio en la absorbancia de la muestra, probada por un haz de baja potencia, como función del flujo de fotones de la excitación primaria. El cambio relativo en el coeficiente de absorción de la muestra viene dado por la Eq. [4.7]. Para tres diferentes longitudes de onda de excitación, se graficó $\alpha_{0} /\left(\alpha_{0}-\alpha_{\mathrm{s}}\right)$ como función de 1/a. De las pendientes y de las coordenadas al origen de las rectas con que se ajustaron los puntos experimentales, se pudo determinar de manera unívoca $\mathbf{p}, \phi_{\mathrm{NP}}, \mathrm{y} \mathbf{B}(\lambda)$. Los signos de la ordenada al origen y de la pendiente de la recta obtenida en cada caso, dependen del signo del factor (B-1). La figura 4.11 muestra los valores experimentales y las correspondientes rectas de ajuste obtenidas. El mejor ajuste resultó ser para: $\mathrm{p}=0.78 \pm 0.08, \phi_{\mathrm{NP}}=0.20 \pm 0.03, \mathrm{~B}(514 \mathrm{~nm})=$ $1.16, \mathrm{~B}(557 \mathrm{~nm})=0.56$ y $\mathrm{B}(562 \mathrm{~nm})=0.50 . \mathrm{B}(550 \mathrm{~nm})=1$ se determinó observando la longitud de onda para la cual la diferencia de absorción era cero. Alrededor de este punto, los cambios en el signo de la señal pueden ser claramente observados. Con el valor de p y el de $\phi_{\mathrm{NP}}$, la eficiencia cuántica de retroisomerización pudo ser evaluada, $\phi_{\mathrm{PN}}=0.16 \pm 0.03$. 


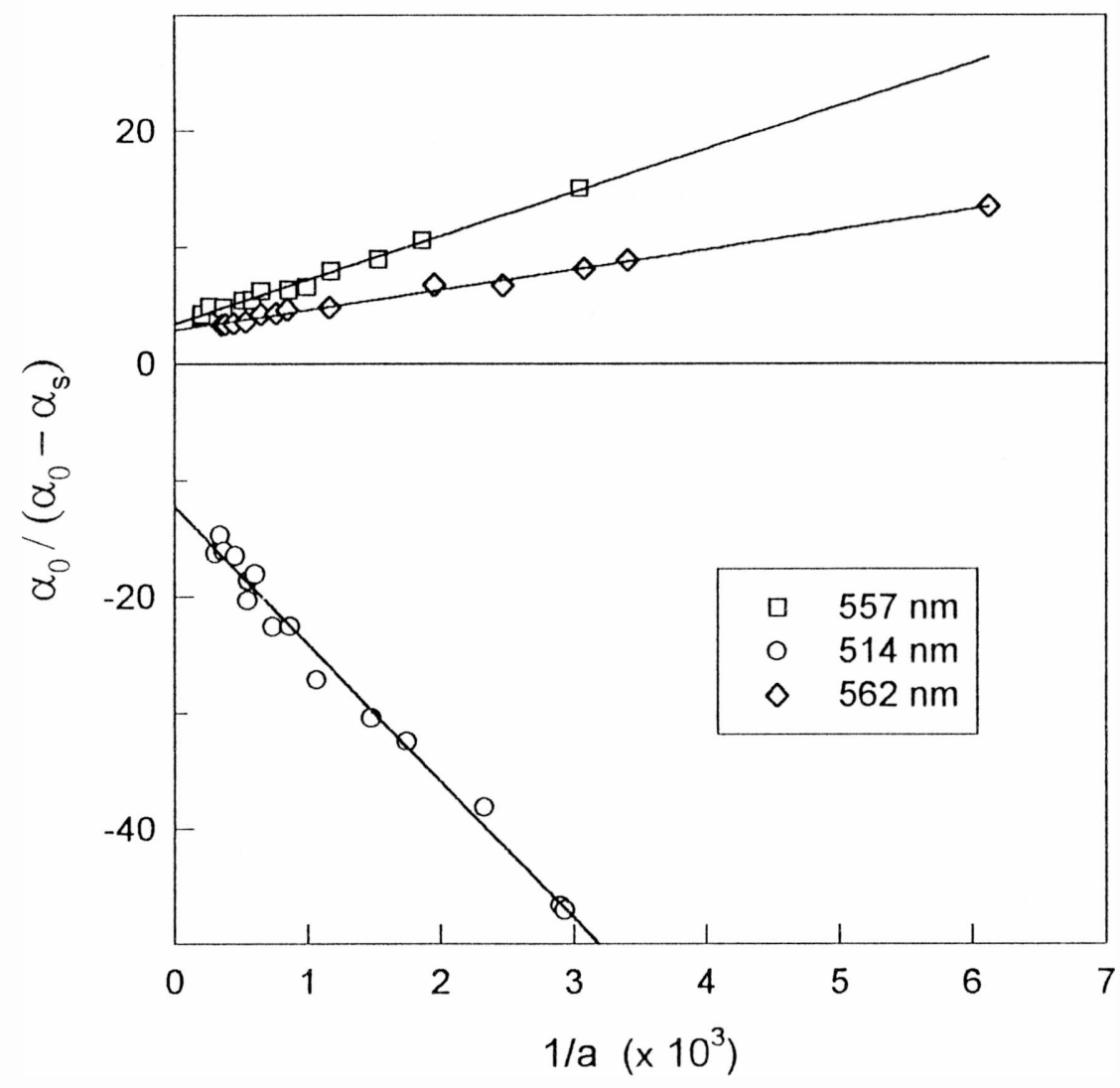

Figura 4.11

Con los valores hallados de B para las diferentes longitudes de onda y el espectro de absorción de la especie normal, medido con un espectrofotómetro Beckman DU-65, se pudo determinar los coeficientes de absorción del fotoisómero en aquellas longitudes de onda, como $\sigma_{\mathrm{p}}=$ B. $\sigma_{\mathrm{N}}$. En la figura 4.12 se muestran los valores de $\sigma_{\mathrm{p}}$ calculados (círculos). Resultados obtenidos de la diferencia de espectros entre ambas especies, usando medidas de flash fotólisis [Ponterini, 1991], permiten recalcular los coeficientes $\sigma_{\mathrm{p}}$. a partir de un valor dado de estos y así comparar con los demás coeficientes calculados. Para ello, se escribe la absorbancia diferencial $\Delta \mathrm{A}$ como

$$
\Delta \mathbf{A} \cdot 2,303 / l=\left(\sigma_{N}-\sigma_{P}\right) \cdot \mathbf{P}_{0}=\sigma_{N} \cdot(\mathbf{l}-\mathbf{B}) \cdot \mathbf{P}_{0}
$$

Luego, para dos longitudes de onda diferentes tenemos, 


$$
\mathbf{B}\left(\lambda_{2}\right)=\mathbf{1}+\frac{\left[\Delta \mathbf{A} / \sigma_{\mathrm{N}}\right]_{\lambda_{2}}}{\left[\Delta \mathbf{A} / \sigma_{\mathrm{N}}\right]_{\lambda_{1}}} \cdot\left(\mathbf{B}\left(\lambda_{1}\right)-\mathbf{1}\right)
$$

Usando esta última ecuación, los valores de $\Delta \mathrm{A}$ tomados de la última referencia, y uno de los valores obtenidos de $B$ (se eligió $B=1$ ), es posible calcular la curva completa para el espectro de absorción de la especie fotoisomérica. El resultado de este cálculo se muestra en la figura 4.12 en linea de puntos. Se observa un buen acuerdo entre el espectro calculado y los puntos obtenidos usando la técnica de absorción.

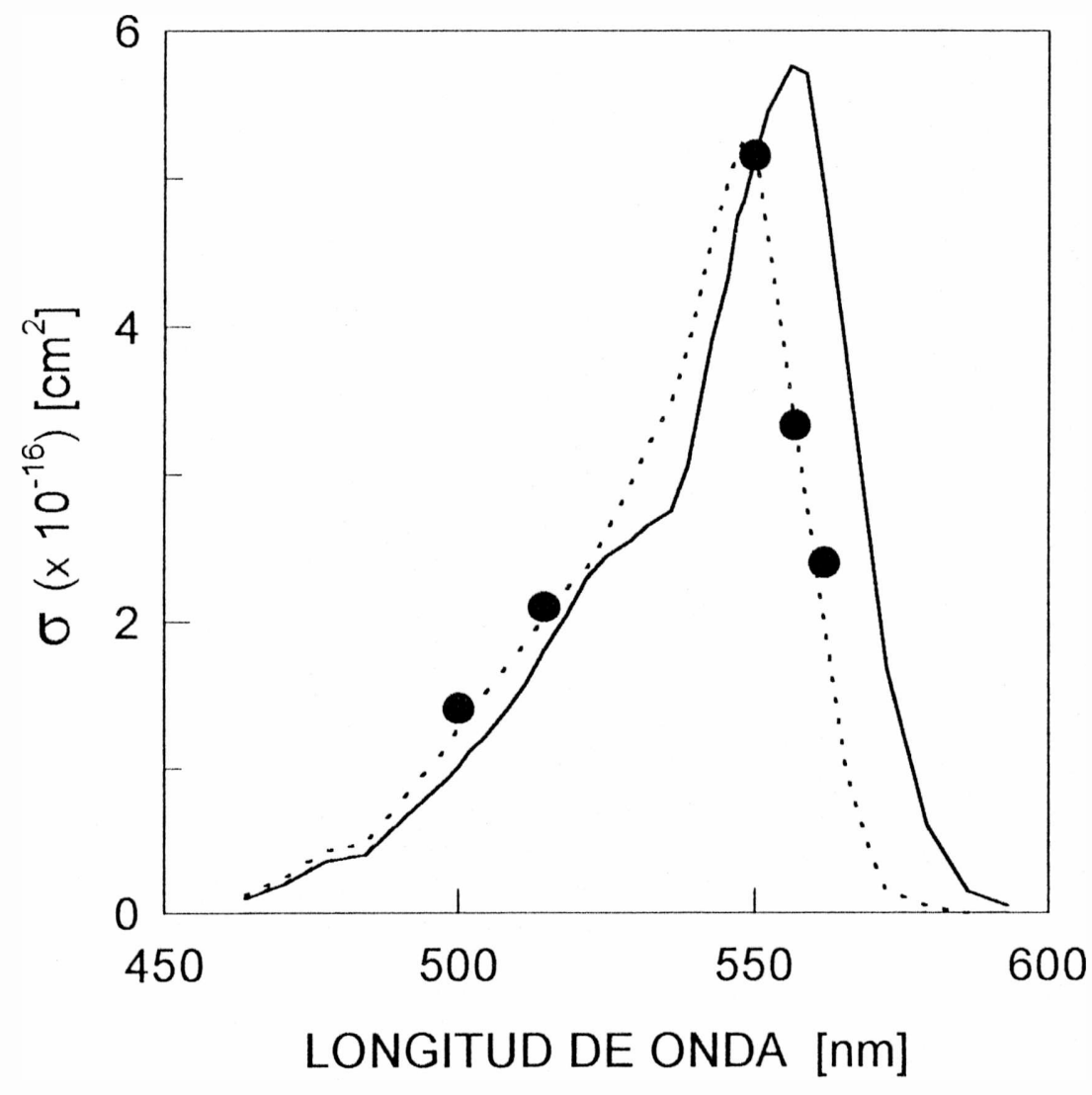

Figura 4.12. Sección transversal de absorción de las especies normal (linea continua) y fotoisómero (linea de puntos). Los circulos corresponden a valores de $\sigma_{p}$ calculados en este trabajo 


\section{MEDIDAS DE FLUORESCENCIA.}

La figura 4.13 muestra el espectro de emisión del DTCI, corregido por la respuesta instrumental del sistema de detección de la fluorescencia. Es de destacar que esta distribución de la emisión no cambia ni con la intensidad ni con la longitud de onda de la excitación. La intensidad de la excitación se varió entre 0.25 y $25 \mathrm{~W} / \mathrm{cm}^{2}$.

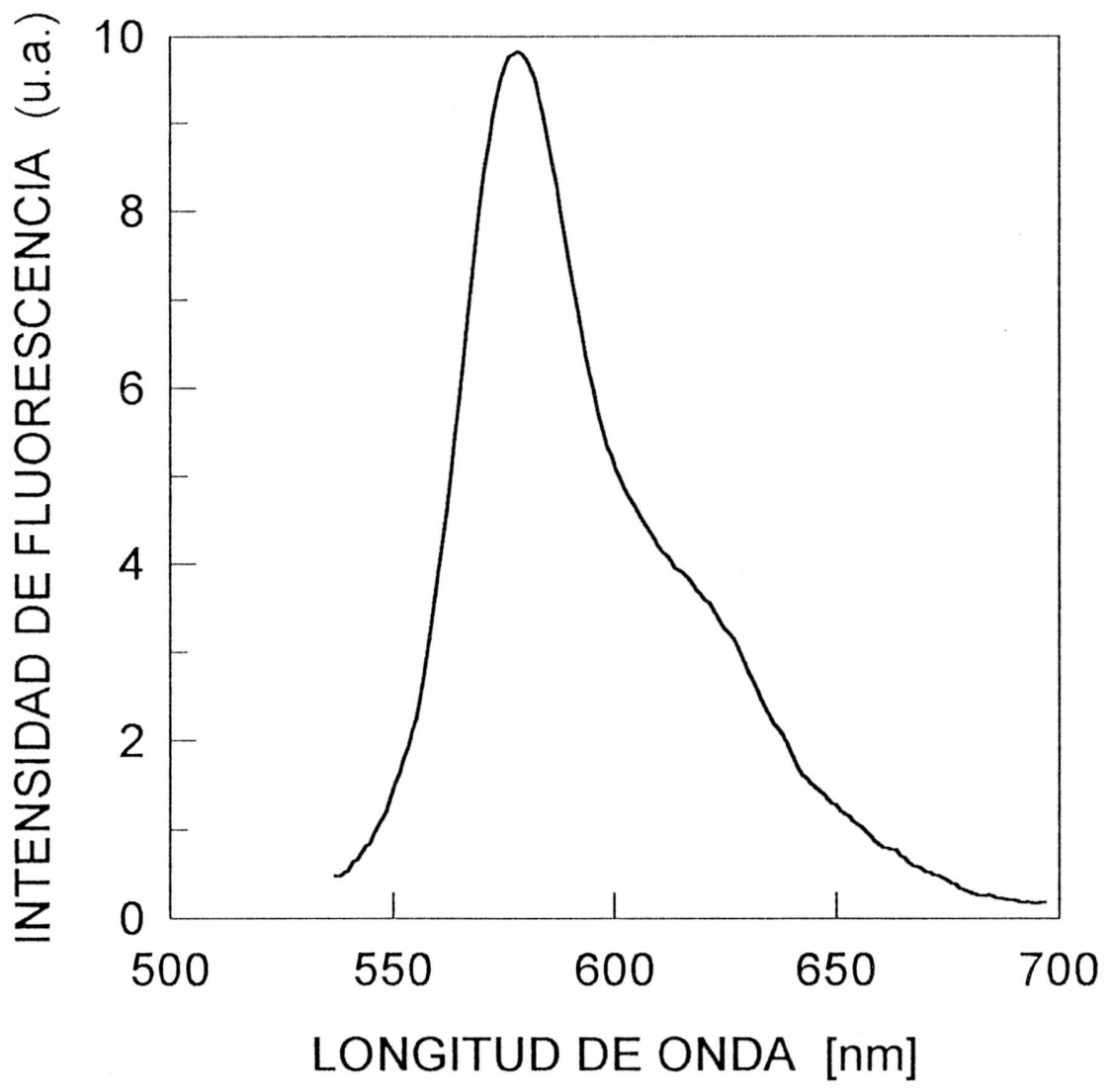

Figura 4.13

En la figura 4.14 se muestra la dependencia con el flujo de fotones absorbidos del cociente entre la intensidad máxima de fluorescencia y aquel flujo, para tres longitudes de onda de excitación diferentes. Habiendo visto que los espectros de absorción de ambas especies isoméricas están muy superpuestos, la intensidad de fluorescencia en cualquier longitud de onda es una combinación de las emisiones de las dos especies. 
Ajustando con la Eq. (4.10) los resultados experimentales mostrados en la Fig. 4. 14, se obtienen valores de $G$ para tres longitudes de onda diferentes. Usando los valores de p y B obtenidos a partir de las medidas de absorción, se logra determinar el cociente de eficiencias cuánticas de fluorescencia $\left(\mathrm{f}\right.$ ) de las dos especies como, $\phi_{\mathrm{r}}^{\mathrm{P}} / \phi_{\mathrm{r}}^{\mathrm{N}} \leq 0.05$. Dado que $\phi_{\mathbf{r}}^{\mathrm{N}}=0.07$ [Aramendía, 1994], la eficiencia cuántica de fluorescencia del fotoisómero resulta ser $\phi_{r}^{p} \leq 0.004$.

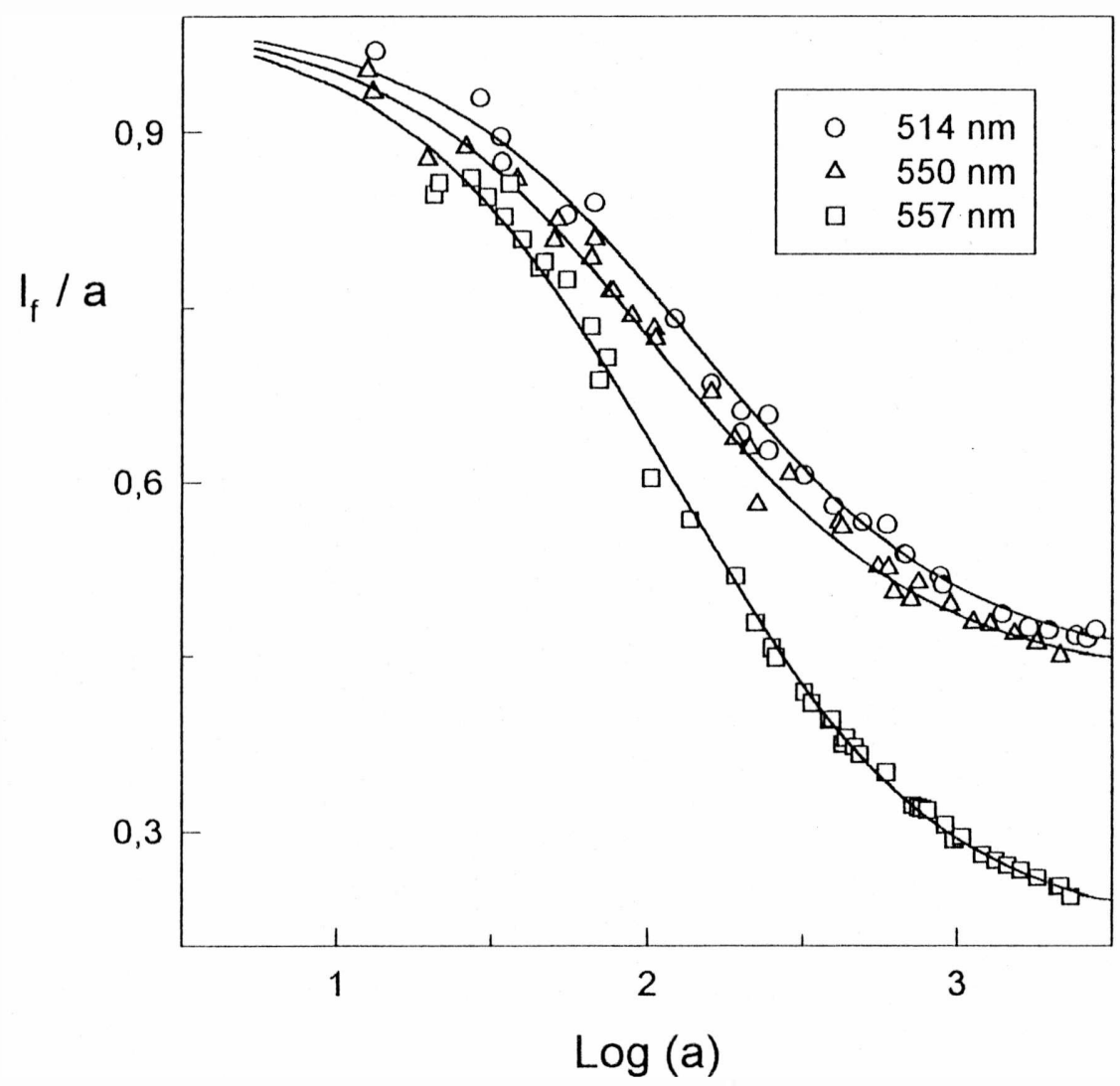

Figura 4.14. Cociente intensidad de fluorescencia/fluencia de excitación (normalizado), como función del logaritmo de esta última. Las curvas que se indican en linea contimua corresponden a ajustes de los puntos experimentales con el uso de la Eq. (4.10).

Los espectros de absorción de ambas especies isoméricas están más superpuestos que en el caso del DODCI, y entonces la absorción de las dos especies en cualquier longitud de onda no puede ser ignorada. La constancia en la forma de la emisión fluorescente frente a cambios en la fluencia de excitación o en la temperatura de la muestra, debe ser asignada al hecho de que la emisión del fotoisómero es despreciable aún respecto del bajo valor de eficiencia de emisión de la especie normal. 


\subsubsection{DOCI}

Como ocurriera con el DTCI, una fuerte superposición de los espectros de absorción del DOCl fue observada. Tampoco su espectro de fluorescencia presenta variaciones con la energía ni con la longitud de onda de excitación, volviéndose dificil entonces obtener información de su especie transiente (fotoisómero). La utilización de las técnicas de absorción y fluorescencia en condiciones de equilibrio fotoestacionario, permitió estudiar el comportamiento relativo de la especie fotoisomérica respecto de la normal y determinar así parámetros fotofisicos y propiedades espectroscópicas de esta molécula. Para la excitación de este compuesto se utilizaron las lineas de un láser de Argón, siguiendo el arreglo experimental ya descripto para el DTCI.

De las medidas de absorción en función de la fluencia de excitación, y en acuerdo con el modelo de fotoisomerización propuesto para estos colorantes, se obtuvo la razón entre eficiencias de isomerización (p) y el cociente entre secciones eficaces de absorción de ambas especies, para dos longitud de onda de excitación. De las medidas de fluorescencia en conjunto con la de absorción se determinó la eficiencia de fluorescencia del fotoisómero

En la figura 4.15 se muestra el resultado experimental de medir la absorción diferencial. El hecho de que las rectas de ajuste tengan signos de sus pendientes y ordenadas al origen opuestos, tiene que ver con el valor relativo entre $\sigma_{\mathrm{p}}$ y $\sigma_{\mathrm{N}}$. La absorción del fotoisómero es menor que la de la especie normal para $476.5 \mathrm{~nm}$, y mayor para la otra longitud de onda de excitación. Independientemente de la fluencia de excitación, la diferencia de absorción $\left(\alpha_{0}-\alpha_{s}\right)$ resultó ser muy próxima a cero en $488 \mathrm{~nm}$. Ello indica que $\sigma_{\mathrm{p}} \cong \sigma_{\mathrm{N}}(\mathrm{B} \cong 1)$ en esta longitud de onda.

El mejor ajuste de los puntos experimentales se obtuvo con: $\mathrm{p}=1.2, \mathrm{~B}(476.5)=$ $0.70, \mathrm{~B}(496.5)=1.53$ y $\phi_{\mathrm{NP}}=0.34$. Con los valores de $\sigma_{\mathrm{N}}$ medidos en el espectrofotómetro, y chequeados con los que aparecen en la literatura [Aramendía, 1994], la sección transversal de absorción del fotoisómero, para las dos longitudes de onda de excitación, resultó: $\sigma_{\mathrm{p}}$ $(476.5 \mathrm{~nm})=2.69 \times 10^{-16} \mathrm{~cm}^{2}$ y $\sigma_{\mathrm{p}}(496.5 \mathrm{~nm})=5.13 \times 10^{-16} \mathrm{~cm}^{2}$. Estos valores están en un acuerdo cualitativo con el corrimiento observado del espectro de absorción hacia longitudes de onda largas, hallado en un trabajo previo en el que un sistema similar es analizado a bajas temperaturas, sin que se llegue a resolver los espectros de ambas especies de esta molécula [Ponterini, 1991]. Usando el valor encontrado de p y $\phi_{\mathrm{NP}}$, la eficiencia de retroisomerización calculada es, $\phi_{\mathrm{PN}}=0.41\left(\right.$ a $25^{\circ} \mathrm{C}$ ).

Debido a la fuerte dependencia de las constantes fotofisicas con la temperatura y la velocidad de flujo de la solución, fue necesario tener especial cuidado en el control de estos parámetros. En la figura 4.16 se puede apreciar el decaimiento de la señal de absorción del fotoisómero, tal como se registró en las medidas de absorción diferencial. El tiempo que permanece el fotoisómero en la región iluminada depende de la velocidad con que se 
renueva la muestra de aquella región $(w)$, y de la constante de retroisomerización térmica (k). El valor medido de este tiempo fue $13.3 \mathrm{~ms}\left(\mathrm{k}+\mathrm{w}=75 \mathrm{~s}^{-1}\right)$. Luego, a temperatura ambiente se observa una fuerte influencia de w sobre este tiempo, dado que el valor de $k$ es mucho más bajo $\left(\mathrm{k}=4 \mathrm{~s}^{-1}\right.$, Aramendía, 1994).

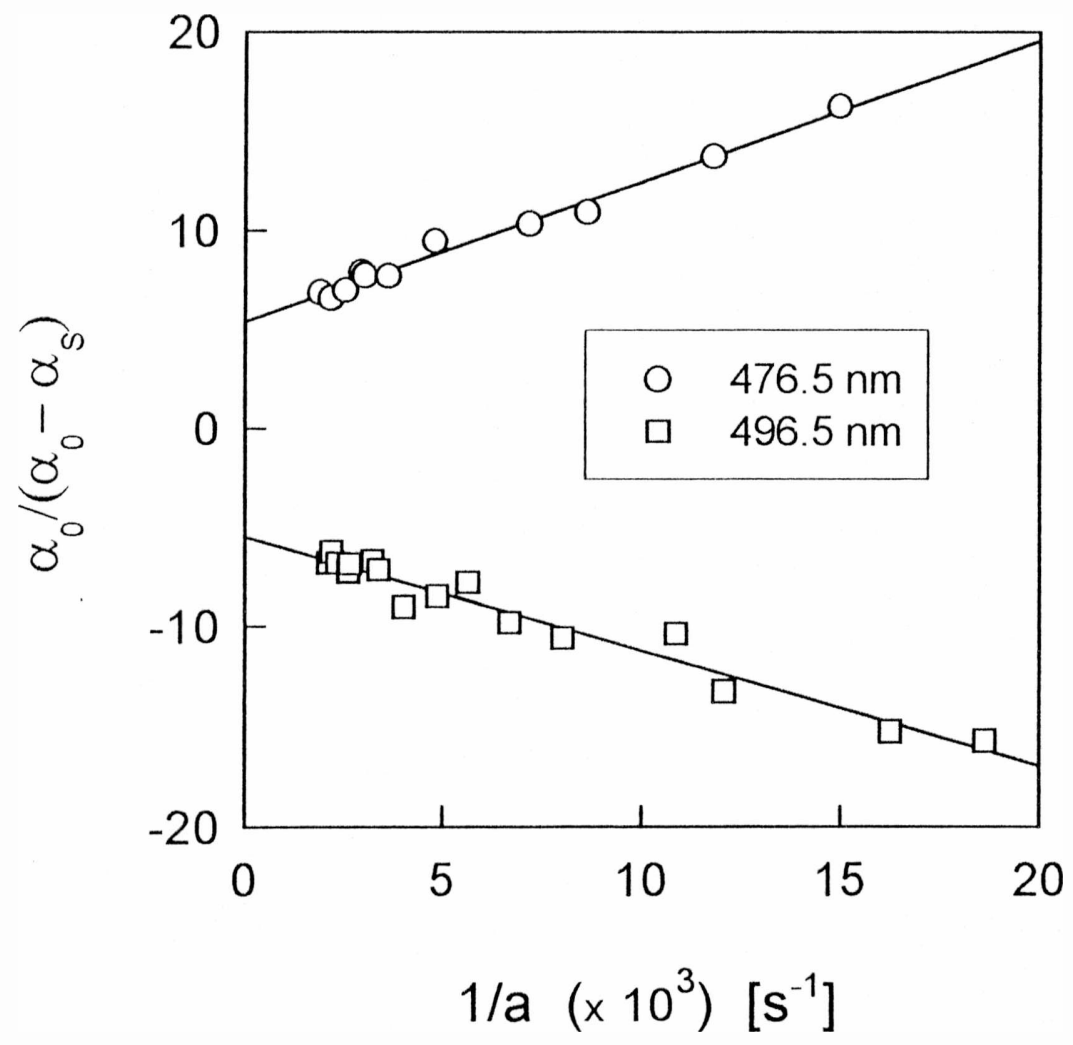

Figura 4.15

A diferencia de las medidas realizadas en el estudio de los dos colorantes anteriores (DODCI y DTCl), ahora la absorción y la fluorescencia fueron medidas simultáneamente. El mismo esquema experimental de la figura 4.10 permite seguir al mismo tiempo cambios en la absorbancia y en la fluorescencia de la muestra, respecto de la fluencia de excitación. Mediante un programa de adquisición de datos (ADA3) desarrollado en el Centro de Investigaciones Ópticas, las señales de absorción y fluorescencia son registradas en forma simultánea. 


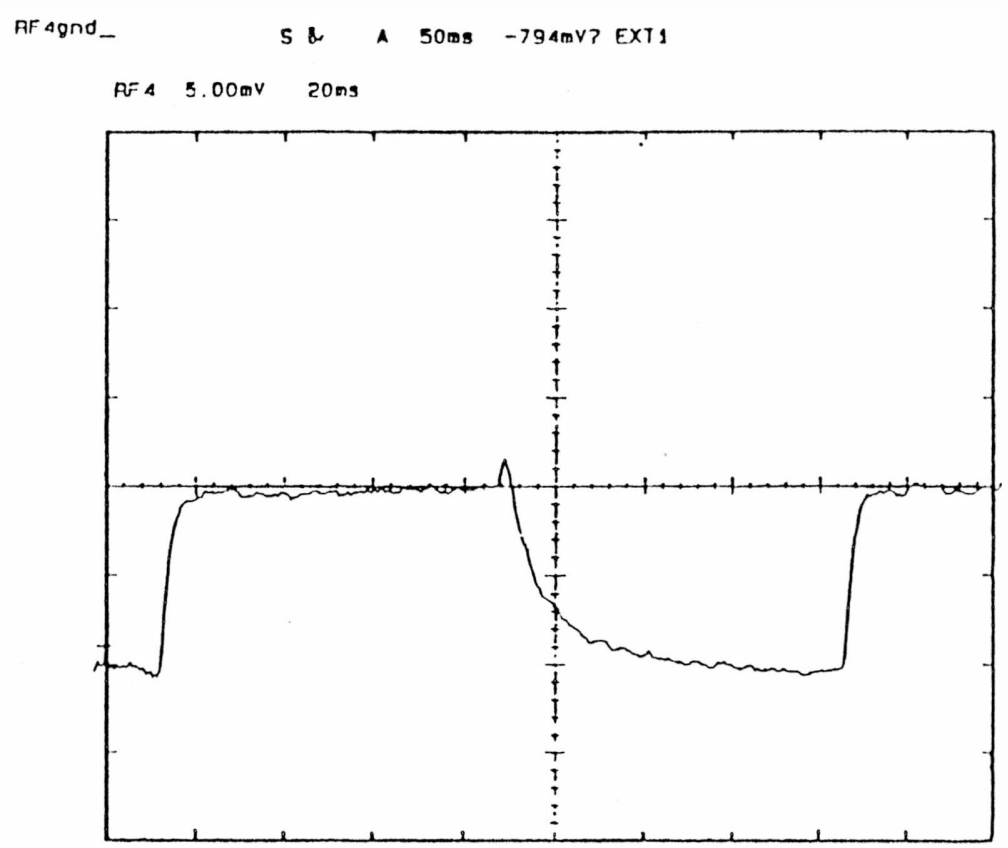

Figura 4.16. Senal de absorción modulada por la frecuencia de encendido-apagado de la excitación primaria. Cuando se interrumpe la exciración puede apreciarse como se desactiva la población del fotoisómero $\left(P_{0}\right)$, recuperdudose la imtensidad del haz de prueba transmitida a través de la muestra.

Un análisis de las expresiones que resultan para la absorción y la fuorescencia por separado, revela que el cociente de las mismas expresiones brinda información de los parámetros buscados y es independiente de la intensidad de excitación. Luego el ajuste se vuelve más sencillo. La intensidad total de fluorescencia (Eq. 4.10) puede ser escrita en función de la fracción de fotoisómero $\left(\mathbf{x}_{\mathbf{p}}\right)$ como,

$$
\frac{\Gamma}{\Gamma-\frac{I_{r}}{a}}=\frac{1}{(1-B \cdot f) \cdot x_{p}}
$$

donde $\Gamma$ es una constante que depende de la longitud de onda de excitación, y los demás parámetros ya fueron definidos anteriormente. Un valor adecuado de $\Gamma$ debería permitir que la representación de los datos de fluorescencia adoptasen la forma de una línea recta cuando el miembro de la izquierda en la ecuación (4.20) es graficado en función de 1/a. (Recordar la expresión de $x_{\mathbf{p}}$ en términos de 1/a, Eq. 4.2). En la figura 4.17 puede apreciarse el resultado de ajustar la constante $\Gamma$ a tal fin, para las medidas de fluorescencia en $476.5 \mathrm{~nm}$

Haciendo el cociente de las ecuaciones (4.6) y (4.20) resulta, 


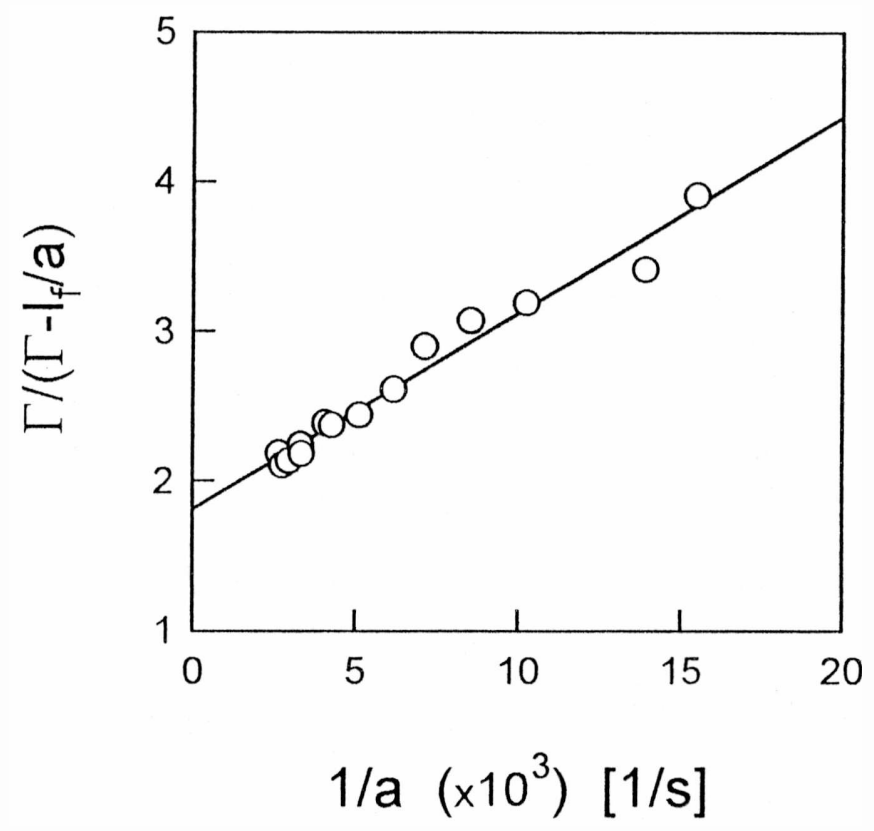

Figura 4.17

$$
\frac{\alpha_{0}-\alpha_{s}}{\alpha_{0}} / \frac{\frac{I_{f}}{a}-\Gamma}{\Gamma}=(B-1) /(\text { B.f }-1)
$$

Midiendo entonces la absorción diferencial y la intensidad de fluorescencia en función de la fluencia de excitación, haciendo luego el cociente absorción/fluorescencia, habiendo previamente linealizado la representación de la fluorescencia, se obtiene un valor (segundo miembro de la Eq. (4.21)) que depende de la longitud de onda de excitación a través de $B$, pero que es independiente de la fluencia de excitación. La figura 418 muestra los resultados obtenidos para las dos longitudes de onda con las que se trabajó (las mismas de absorción). Con los valores de B obtenidos de las medidas de absorción y las ordenadas al origen del cociente absorción/fluorescencia ((B-1)/(B.f-1)), el valor del cociente de eficiencias cuánticas de fluorescencia $(f)$ fue determinado. $f=0.09$. Teniendo en cuenta que la eficiencia cuántica de fluorescencia de DOCI en etanol es 0.05 a $21^{\circ} \mathrm{C}$ [Ponterini, 1992], obtenemos para la eficiencia de fluorescencia del fotoisómero $\phi_{r}^{\prime}=4.5 \times 10^{-3}$. 


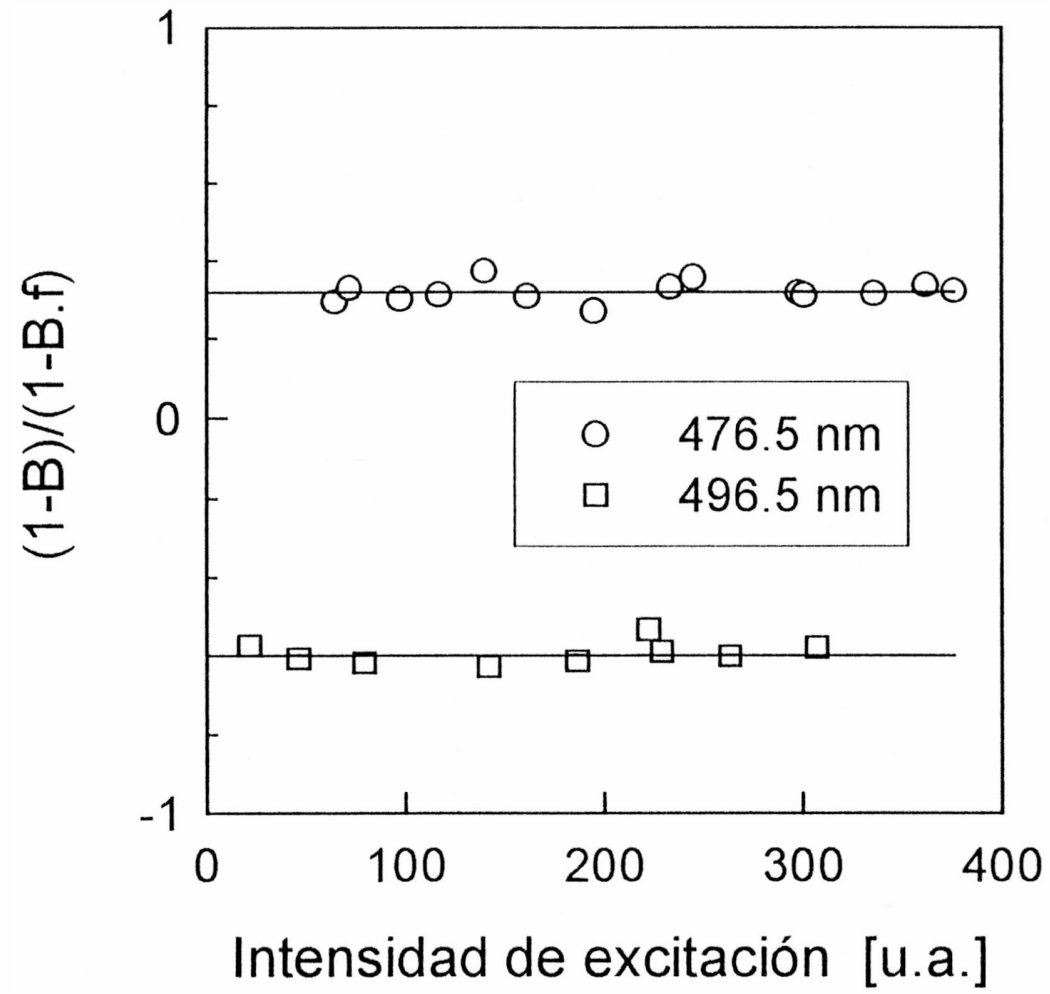

figura 4.18

La cinética de esta molécula puede ser ahora analizada como sigue. Si la conversión interna directa de la especie normal puede despreciarse [Aramendía, 1994], la eficiencia cuántica del pasaje $S_{1}^{N} \rightarrow t$ (ver figura 2.4), es $\phi_{N_{t}}=1-\left(\phi_{f}^{N}+\phi_{C l}^{N}\right) \cong 0.95$. Habiéndose determinado $\phi_{\mathrm{NP}}=\phi_{\mathrm{N}} \cdot \mathrm{r}=0.34$, donde $\mathrm{r}$ representa la fracción de moléculas que decaen desde el estado twisted al fundamental del fotoisómero (ver figura en la página 60), resulta para $r$ un valor igual a 0.36 .

$\phi_{\mathrm{PN}}=\phi_{\mathrm{Pt}} \cdot(1-\mathrm{r})$, luego $\phi_{\mathrm{Pt}}=0.64$. La eficiencia de fluorescencia despreciable del fotoisómero determina que la conversión interna directa de esta especie resulte ser $\phi_{\mathrm{Cl}_{\mathrm{i}}}^{\mathrm{P}}=1-\left(\phi_{\mathrm{Pt}}+\phi_{\mathrm{f}}^{\mathrm{p}}\right) \cong 0.36$.

La metodología adoptada permite así obtener parámetros fotofisicos de las especies fotoisoméricas, relativos a valores correspondientes de las especies normales. Es de destacarse en el caso de la especie fotoisomérica de esta molécula, el bajo resultado obtenido de su eficiencia cuántica de fluorescencia, tal como ocurriera con el DTCI. 


\subsubsection{DTDCI}

La fotoisomerización de este colorante ha sido estudiada detalladamente en el pasado, utilizándose para ello distintas técnicas [Kaliteevskaya, Bilmes (1989)]. Los espectros de absorción de ambos isómeros, su especie normal y su fotoisómero, han sido determinados, observándose una fuerte superposición de los mismos. Más recientemente han aparecido trabajos en los que se vuelve a discutir la fotoisomerización de este compuesto, incluyéndose la formación de un segundo fotoisómero para fluencias altas de excitación [Razumova, Awad, Vaveliuk (1996)]. Buscando información sobre las características luminiscentes de su especie transiente, el mismo estudio que se realizara con DOCI se hizo con este colorante. A diferencia de las anteriores cianinas, las soluciones de este compuesto se prepararon con metanol, dado que la mayor parte de la información disponible sobre esta molécula tenía base en este alcohol. Se excitó con un láser de colorantre (DCM) en 632 y $652 \mathrm{~nm}$. Haciendo el cociente absorción/fluorescencia, tal como se describió en el caso del DOCl, y usando valores de B tomados de la literatura [Bilmes], fue posible determinar la razón entre las eficiencias cuánticas de fluorescencia de la especie normal y su fotoisómero.

La figura 4.19 muestra el resultado de hacer el cociente absorción/fluorescencia para la excitación en $652 \mathrm{~nm}$. Usando $\mathrm{B}(652 \mathrm{~nm})=0.63$, se determinó el cociente de eficiencias de fluorescencia ( $C<0.1$ ). Dado lo pequeño de este valor, la eficiencia de la especie fotoisomérica resulta una vez más ser despreciable. Ello se corresponde con el hecho de que

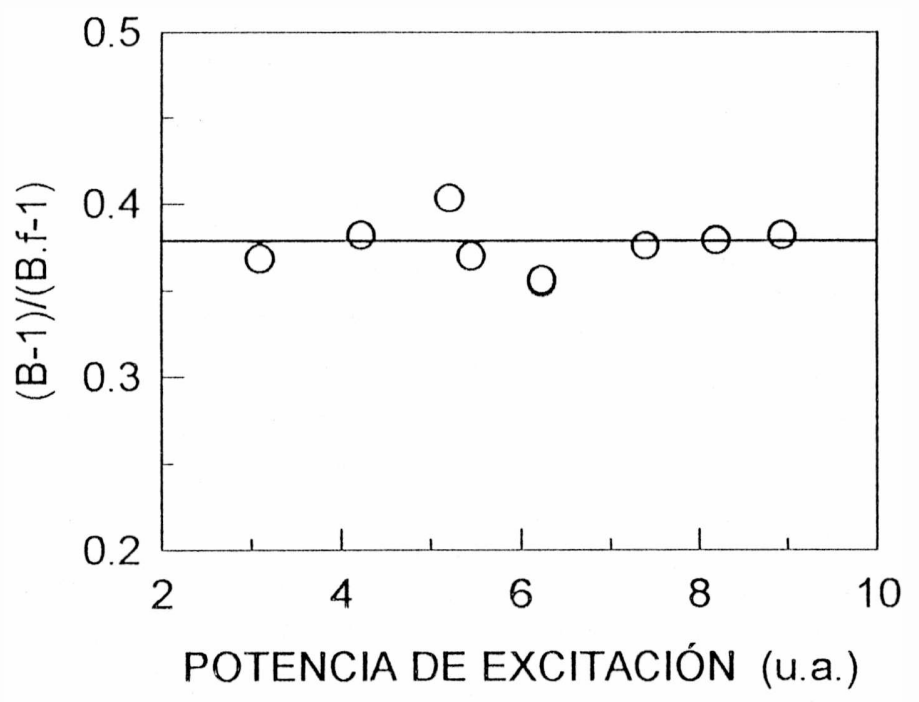

Figura 4.19 
los espectros de fluorescencia de esta molécula no mostraron cambios con la fluencia de excitación. Coincidiendo con el comportamiento hallado para las especies fotoisoméricas del DTCI y DOCI, su eficiencia de fluorescencia es demasiado pequeña $\left(\phi_{i}^{\mathrm{P}}<0.03\right)$ como para poder observarse la presencia de su espectro.

\subsection{CONCLUSIÓN.}

En resumen, como resultado de la aplicación de las técnicas mencionadas, se logró obtener información de la especie transiente del DODCI, minimizando la influencia de su forma normal. De esta manera se pudo obtener el espectro de fluorescencia de su fotoisómero y a partir de este, la eficiencia de fluorescencia, el tiempo de vida y la población de su estado fundamental, todos en función de la temperatura.

En el caso de las demás cianinas estudiadas, los espectros de absorción de ambas especies isoméricas aparecen más superpuestos que en el DODCI, y entonces la absorción de las dos especies en cualquier longitud de onda no puede ser ignorada. La constancia en la forma de la emisión fluorescente frente a cambios en la fluencia de excitación o en la temperatura de la muestra, debe ser asignada al hecho de que la emisión del fotoisómero es despreciable, aún respecto del bajo valor de eficiencia de emisión de sus especies normales.

La metodología adoptada permitió entonces obtener parámetros fotofisicos de las especies fotoisoméricas, en particular datos de fluorescencia que serán utilizados en lo que sigue. Salvo el caso del DODCI, por el comportamiento espectral de sus especies normal y fotoisomérica, el comportamiento global de estas cianinas es muy similar entre sí. 


\section{CAPÍtULO 5. DESARROLLO EXPERIMENTAL Y RESULTADOS DE ANISOTROPÍA.}

\subsection{INTRODUCCIÓN}

Habiendo analizado las propiedades cinéticas y características espectroscópicas de las cianinas ya mencionadas, se empleó la técnica de polarización de la luminiscencia con el fin de discernir la estructura isomérica de estas moléculas en solución. Midiendo la anisotropía en estado estacionario de la fluorescencia que se genera bajo excitación luminosa, se pudieron determinar tiempos de difusión rotacional para las diferentes especies. Los resultados están vinculados directamente con la estructura isomérica que adopta la molécula en solución y con las interacciones de esta con el solvente. Asignando determinados modelos moleculares a las especies estudiadas y analizando el problema hidrodinámico de la difusión rotacional con diferente condición de vínculo soluto-solvente, se logró predecir el comportamiento rotacional de estas moléculas.

Tal como fuera señalado en el capítulo 4 , la posibilidad de separar los espectros de fluorescencia de la especie normal de las cianinas estudiadas y del fotoisómero de estas, resulta cierta sólo en el caso del DODCI. Ello se debe a las características particulares de este colorante, cuyos espectros no se hallan tan superpuestos como en las restantes cianinas, y a que la eficiencia de fluorescencia, tanto de su especie normal como de su fotoisómero, resultan importantes. En las dos cianinas de cadena más corta (DTCI y DOCI) no sólo la eficiencia de las especies normales es comparativamente más pequeña, sino que además sus fotoisómeros tienen eficiencias de fluorescencia muy por debajo de las primeras (un orden de magnitud o más). Desafortunadamente las características de estos sistemas impide estudiar la anisotropía de la luminiscencia de sus especies fotoisoméricas.

En lo que sigue, se discutirá en detalle el estudio llevado a cabo en DODCI y posteriormente se resumen en conjunto los resultados que se obtuvieron para la anisotropía de la flluorescencia de las especies normales de las restantes cianinas.

\subsection{ESTUDIO DE LA DIFUSIÓN ROTACIONAL DEL DODCI.}

Con el fin de determinar la estructura isomérica del DODCI en solución, se midió la anisotropía en estado estacionario de su luminiscencia. Resultados experimentales obtenidos siguiendo la polarización resuelta en tiempo [Fleming, \#49], inducían a asignar una conformación di-cis para su forma normal y otra todo-trans para el isómero que se genera bajo excitación luminosa. Este resultado se contradice con las predicciones teóricas acerca de la 
conformación estructural de colorantes con cadena polimetínica, que dan como todo-trans a la especie más estable [Kolesnikov], y con estudios optoacústicos recientes que coinciden con este último resultado [Churio].

Si bien el estudio de la forma normal no presentaba mayores inconvenientes, el de la otra especie sí, debido a la dificultad que existía para separarla de la primera. Tras haberse resuelto este problema en la forma que ya fuera detallada (Cap. 4), la anisotropia en estado estacionario de las emisiones fluorescentes de ambas especies pudieron ser estudiadas separadamente

\section{- DESARROLLO EXPERIMENTAL.}

La anisotropía de la fluorescencia se determinó a partir de las intensidades de emisión observadas con polarización paralela y perpendicular a la dirección de polarización de la luz de excitación. Estas intensidades fueron corregidas en la forma usual [Paladini, Chen], para tener en cuenta la respuesta del equipo experimental. La fluorescencia fue detectada a $90^{\circ}$ con respecto a la dirección del haz de excitación y analizada a través de un polarizador. Un filtro de color se usó para eliminar la luz dispersa del láser.

La preparación de las soluciones, al igual que el control de la temperatura y del flujo de las mismas, se realizó en la forma como se describe en la sección 4.4.1. El circuito por el cual fluye la solución es el mismo antes indicado, como así también el rango de temperaturas estudiado. El montaje experimental se muestra en la figura 5.1. Las emisiones con polarización paralela y perpendicular se midieron alternativamente, en función de la temperatura.

El comportamiento de la especie normal se estudió excitando en forma continua la muestra con baja fluencia ( $F=0.1 \mathrm{~W} / \mathrm{cm}^{2}$ ). Un láser de colorante (Rodamina 110 ) proveyó la luz de excitación en $\lambda=550 \mathrm{~nm}$. El láser se focalizó sobre la celda en un haz de diámetro $0.5 \mathrm{~mm}$. La fluorescencia luego de ser analizada con el polarizador $P_{1}$, se hizo pasar a través del filtro ( $F_{1}$ ) Schott OG-570. Luego de ser colimada, esta luminiscencia se detectó usándose para ello un fotomultiplicador (Hamamatsu R-446) operando en modo corriente. La velocidad con que fluía la solución a través de la celda, se seleccionó para remover el líquido de la zona iluminada en aproximadamente $10 \mathrm{~ms}$, y así evitar la acumulación de fotoisómero en esa región.

Aumentando la potencia del láser de colorante hasta alcanzar una fluencia de excitación mucho mayor ( $\mathrm{F}=100 \mathrm{~W} / \mathrm{cm}^{2}$ ), como ya fuera discutido en el capítulo anterior, se logró transferir aproximadamente toda la población al estado fundamental del fotoisómero. Luego esta población fue excitada con un láser pulsado, sintonizado en su región de absorción, en forma tal que la emisión pulsada que se obtiene corresponde a la fluorescencia del estado excitado $\mathrm{P}_{1}$, libre de la primera especie. El mismo láser pulsado 


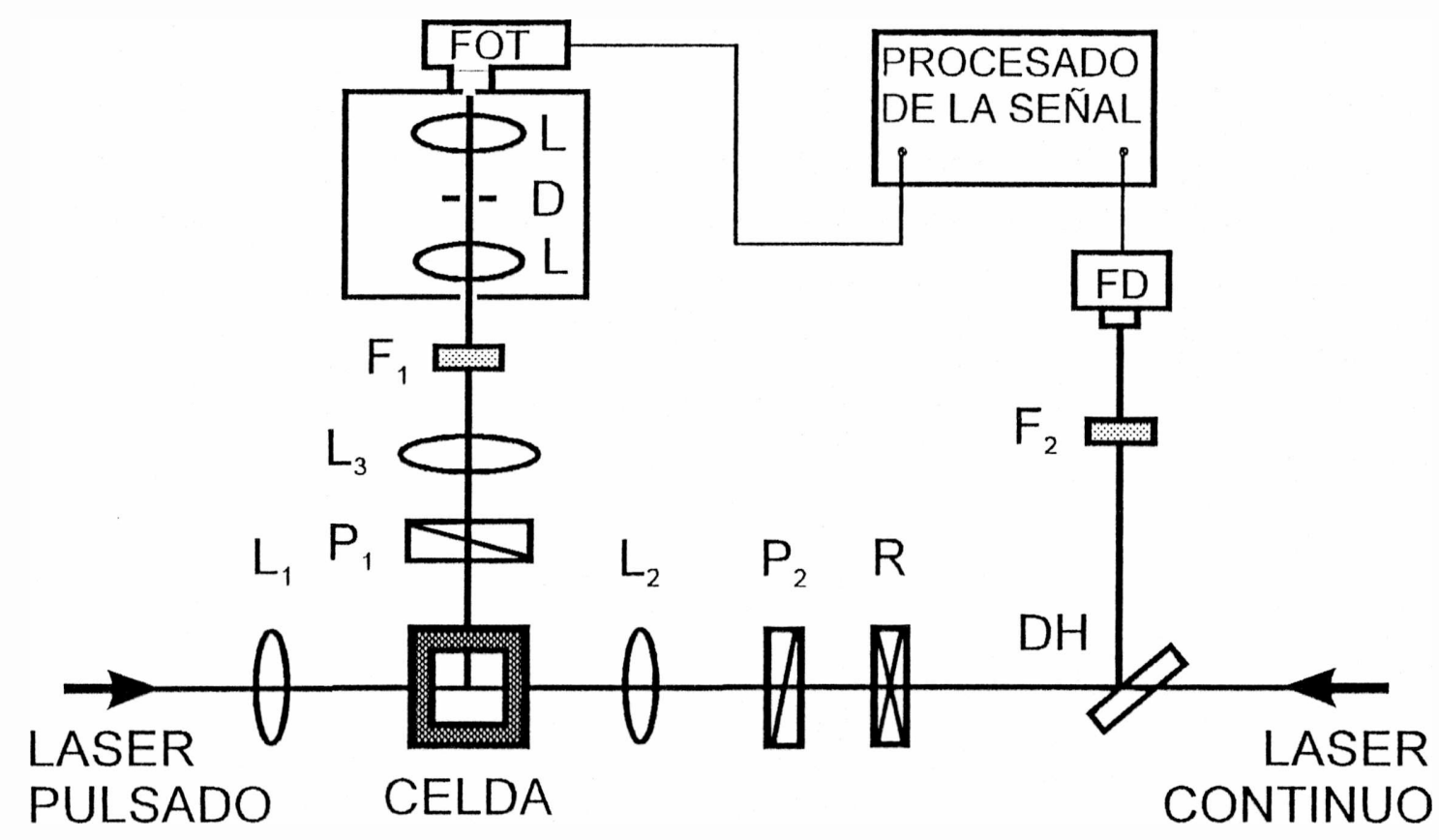

Figura 5.1. Esquema experimental. La notación se corresponde con la de la figura 4.10.

(Chromatix CMX-4) que se usara para estudiar la dinámica de fotoisomerización de este compuesto, es empleado ahora en su estudio de polarización. Utilizando Rodamina 640 como colorante, se excitó la muestra en $\lambda=640 \mathrm{~nm}$. El láser fue nuevamente alineado en el sentido opuesto al láser continuo, y focalizado con una segunda lente en la misma región espacial de la celda donde se estableció la población continua $P_{0}$. Para eliminar ahora la luz dispersa del láser, se usó un filtro Schott OG-665.

La polarización del láser continuo y del pulsado son perpendiculares al plano definido por las direcciones de observación de la fluorescencia e incidencia de los láseres sobre la celda. La emisión volvió a detectarse usándose el mismo fotomultiplicador, pero ahora la emisión pulsada se procesó con un integrador (Boxcar) de baja impedancia.

\section{- RESUltados.}

En la figura 5.2 puede verse la anisotropía de la fluorescencia obtenida para ambas especies del DODCl en etanol, como función del cociente temperatura / viscosidad. Estos resultados se analizarán asumiendo que las especies del DODCI se presentan en dos de las tres estructuras isoméricas que se indican a continuación: todo-trans; 11,12 mono-cis y 8,911,12 di-cis. (Las mismas pueden verse al final del apéndice $\mathrm{C}$ ). Las dos primeras son estructuras planas, en tanto que la di-cis no puede cerrarse en el plano por razones estéricas. 
Se han elegido estas tres estructuras y no otras, por diversos motivos. Como ya fuera mencionado anteriormente, la primera (todo-trans) es la que se espera corresponda a la especie normal en acuerdo con análisis teóricos y evidencia experimental recogida con otros compuestos. La estructura di-cis ha sido propuesta para esta especie en un trabajo previo [Fleming, \#49], y también es indicada en la fotoisomerización del benceno. Por último, la estructura mono-cis elegida asegura una despolarización rápida en virtud de la posición de la cadena polimetínica respecto de los grupos terminales de la molécula. Asumiremos luego en la discusión de los resultados que el dipolo de transición se localiza a lo largo de esta cadena.

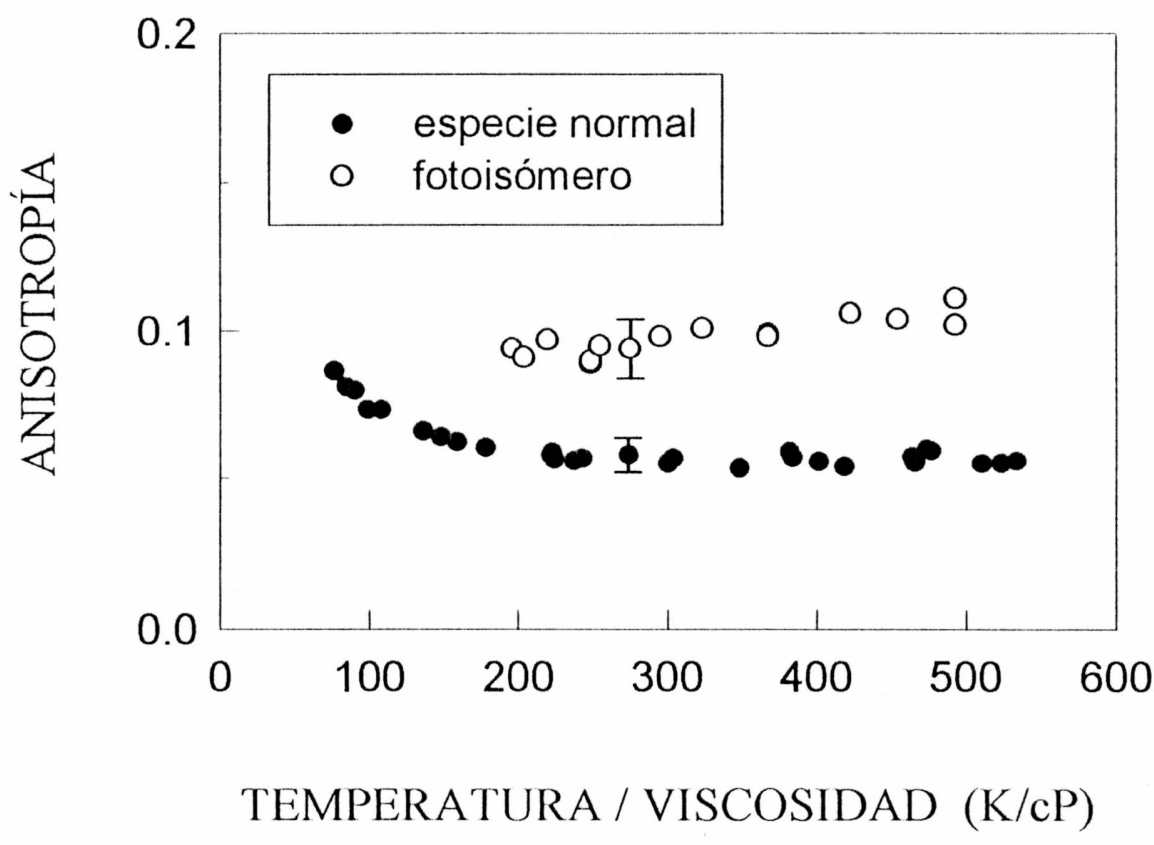

Figura 5.2. Anisotropia de la fluorescencia del DODCI.

Para simular la forma de la molécula, se usó un elipsoide asimétrico en los casos I (todo-trans) y II (mono-cis). Un esferoide oblado representa la estructura más compacta dicis (III). La forma y dimensiones de los elipsoides elegidos, son consistentes con las estructuras propuestas ( ver figura 5.3) y con el volumen de van der Waals [Edward] de la molécula. Para el elipsoide asimétrico, la longitud del eje más largo se tomó igual a la separación entre los dos hidrógenos más distantes entre sí, incluyéndose el radio de van der Waals de estos átomos. La longitud del eje más corto se eligió para lograr el mejor ajuste 
visual desde una vista lateral del modelo de llenado espacial. El restante eje es calculado en orden de hacer el volumen del elipsoide igual al volumen de van der Waals de la molécula. Si bien esta última elección parece estar de acuerdo con la representación de la molécula mostrada en la figura 5.3, es evidente que representar la molécula con una forma elipsoidal compacta subestima su volumen efectivo de difusión. Sin embargo, ha sido nuestra intención

\section{I dodci todo-trans}
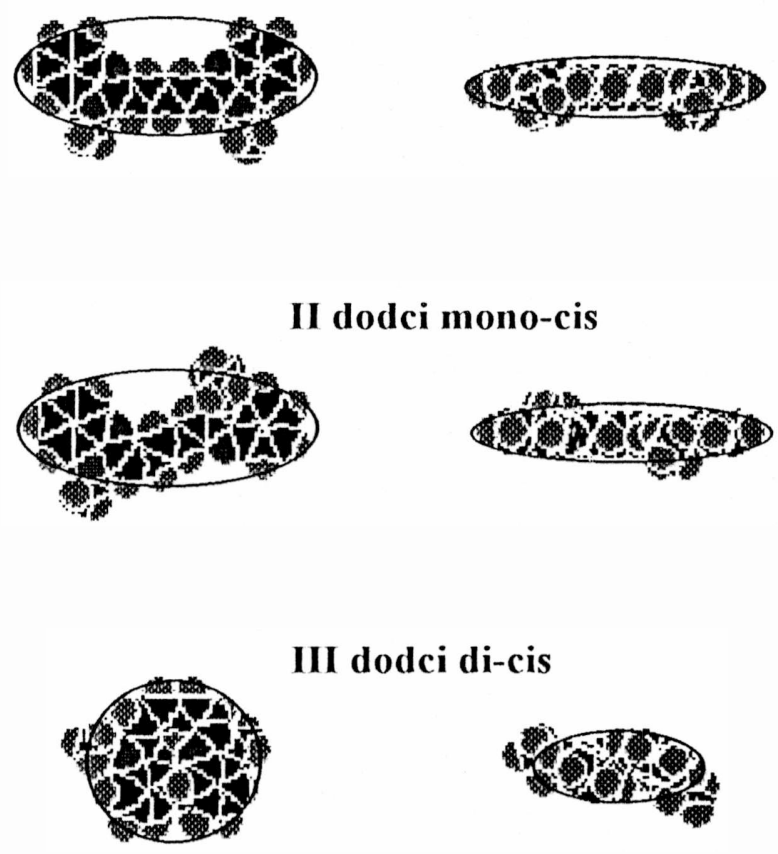

Figura 5.3

trabajar con este mínimo volumen posible, para así examinar desde este punto de partida las predicciones teóricas de ambas condiciones de contorno hidrodinámicas (stick-slip). Como veremos más adelante, esta elección permite descubrir la fuerte inconsistencia que surge entre el modelo hidrodinámico stick y los datos experimentales aquí obtenidos. Para el rotor oblado, el eje más largo se eligió como antes y el eje más corto se calculó de nuevo en orden de ajustar el volumen al de van der Waals.

En la figura 5.4, junto con los datos experimentales, se muestran curvas teóricas para la despolarización de la fluorescencia correspondientes a las distintas estructuras propuestas, con diferente condición de contorno hidrodinámica (stick-slip). En el cálculo de las mismas, 
se utilizaron para representar al tiempo de vida de cada una de las especies del DODCI, los parámetros de Arrhenius hallados en el capítulo anterior y que se resumen en el apéndice $C$.

En la obtención de las curvas se asume que los momentos de transición para absorción y emisión de esta molécula, son colineales. Este hecho puede ser justificado porque la anisotropía del DODCI a bajas temperaturas o en solventes de viscosidad muy alta, es cercana al valor 0.4 [Anfinrud]. Similarmente, en estudios de anisotropía resuelta en tiempo [Fleming], se señala que la anisotropía límite de esta molécula debería ser próxima a aquella característica de momentos de transición colineales

Como la absorción y la emisión están representadas por una transición electrónica $\pi \pi^{*}$, se asume además que ambos momentos de transición yacen en el plano de simetría formado por los dos ejes más largos del elipsoide. Sea $\theta$ el ángulo entre el dipolo de transición y el eje mayor del elipsoide. Es precisamente este ángulo el que hace la diferencia entre el modelo adoptado para representar a la estructura todo-trans y aquel elegido para el mono-cis. Esto es, la orientación del dipolo de transición respecto del eje mayor del elipsoide. En la primera, dicho dipolo se considera paralelo al eje mayor, mientras que para la forma II el dipolo se asume que forma un ángulo de $\pi / 8$ radianes con aquel eje

Las dimensiones de los dos elipsoides usados en el modelado de las estructuras moleculares y los tiempos de difusión rotacional alrededor de cada uno de sus ejes principales, calculados siguiendo los procedimientos indicados en el capítulo 3 , se muestran en la tabla 5.1

Tabla 5.1.

\begin{tabular}{||c|c|c|c|c|c|c|c|c||}
\hline Modelo & semiejes $(\boldsymbol{\AA})$ & $\boldsymbol{\theta}$ & $\mathbf{A}_{1}$ & $\mathbf{A}_{\mathbf{2}}$ & $\mathbf{A}_{\mathbf{3}}$ & $\mathbf{a}_{\mathbf{1}}\left(\mathbf{n s}^{-\mathbf{1}}\right)$ & $\mathbf{a}_{\mathbf{2}}\left(\mathbf{n s} \mathbf{1}^{-1}\right)$ & $\mathbf{a}_{\mathbf{3}}\left(\mathbf{n s}^{-\mathbf{1}}\right)$ \\
\hline \hline I stick & $10-4-2$ & $0^{\circ}$ & 0.00008 & 0.39992 & - & 8.56 & 3.41 & - \\
\hline I slip & $10-4-2$ & $0^{\circ}$ & 0.002 & 0.398 & - & 30.5 & 7.5 & - \\
\hline II stick & $10-4-2$ & $22.5^{\circ}$ & 0.124 & 0.002 & 0.271 & 4.77 & 8.58 & 3.41 \\
\hline II slip & $10-4-2$ & $22.5^{\circ}$ & 0.124 & 0.004 & 0.273 & 14.67 & 30.17 & 7.46 \\
\hline III stick & $6-6-2.2$ & $0^{\circ}$ & 0.1 & 0.3 & - & 7.55 & 6.53 & - \\
\hline III slip & $6-6-2.2$ & $0^{\circ}$ & 0.4 & - & - & 14.85 & - & - \\
\hline
\end{tabular}

Todos los coeficientes fueron calculados a temperatura ambiente $(293 \mathrm{~K})$. Lil solvente es etanol absoluto. Oes el ángulo entre el dipolo de transición y el eje mayor del elipsoide 


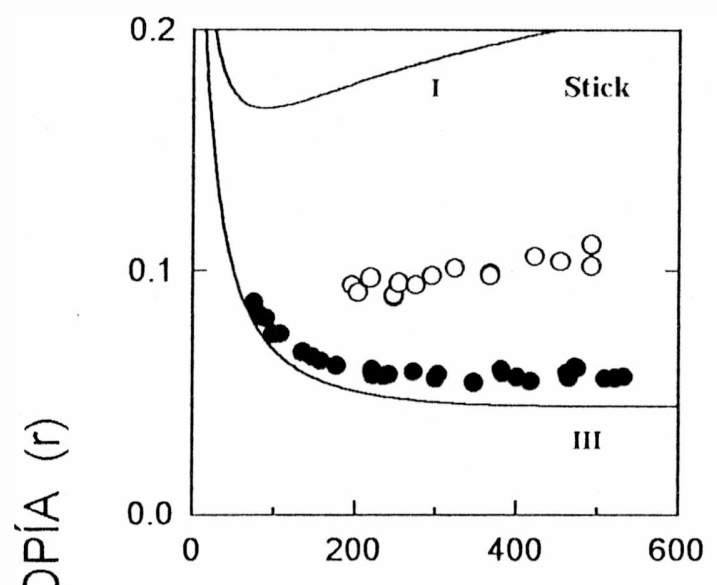

(a)

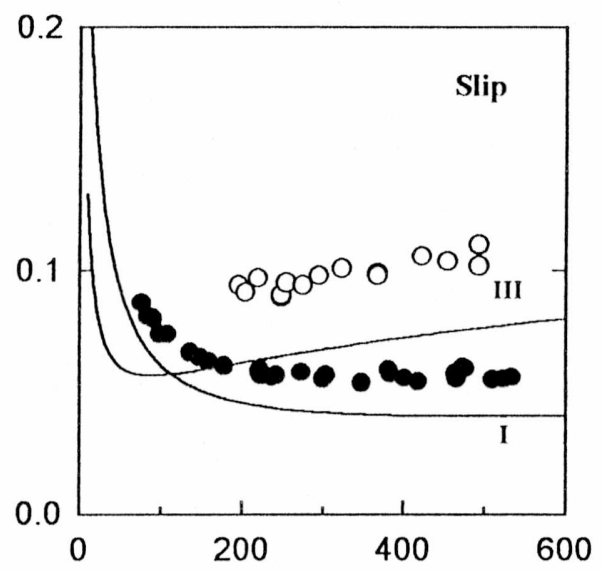

(c)

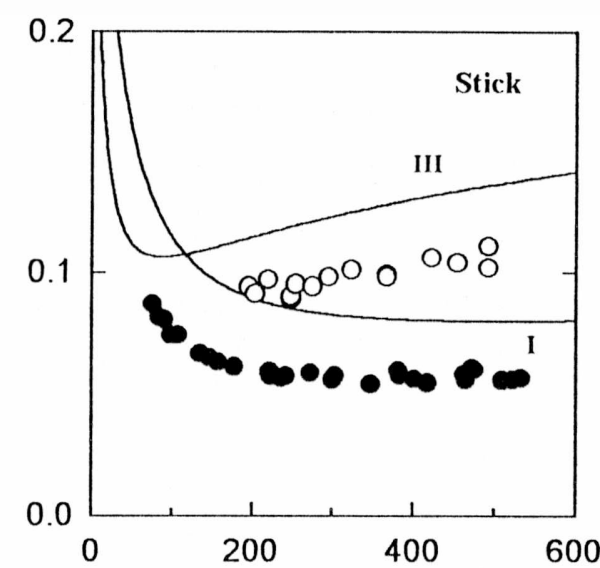

(b)

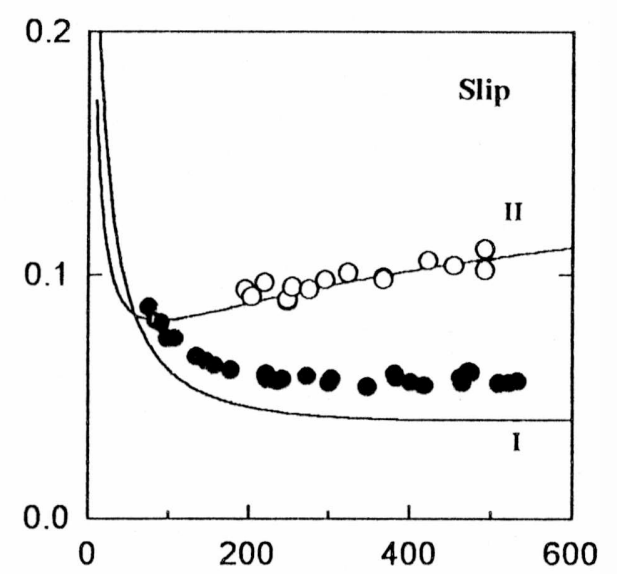

(d)

\section{TEMPERATURA / VISCOSIDAD (K/cP)}

Figura 5.4. Curvas teóricas de la anisotropia que predicen los modelos hidrodinámicos, para las distintas estructuras mostradas en la figura 5.3. (a) Condición stick, se supone para $N$ la estructura di-cis (III) y para el fotoisómero la todo-trans (I). (b) Condición stick, $N$ todo-trans, $P$ di-cis. (c) Se cambió la condición stick por slip, igual que en (b) $N$ se supone todo-trans y P di-cis. (d) Se mantiene la condición slip. N todo-trans y el fotoisómero pasa a estar representado por la estructura mono-cis (II). Los circulos negros corresponden a los valores de anisotropía medidos para la especie normal (N) y los circulos blancos a los de la especie fotoisomérica (P). 
Para el elipsoide asimétrico que representa a la especie todo-trans, el decaimiento de la anisotropía calculado resultó ser prácticamente monoexponencial, con ambas condiciones de contorno hidrodinámicas. Aparecen dos decaimientos exponenciales, pero uno de ellos se puede despreciar a causa del factor pre-exponencial que lo acompaña. Para el modelo de la especie mono-cis, aparecen dos decaimientos exponenciaies dominantes, pero al igual que en el caso ya discutido de un elipsoide oblado (sección 3.2.1.), estos dos decaimientos son muy similares y pueden describirse mediante una sola exponencial. Por su parte, el rotor oblado presenta dos decaimientos con condición stick y sólo uno para slip

De acuerdo con estos resultados, la expresión de la anisotropía en estado estacionario para los modelos considerados, puede ser expresada ya sea en forma exacta o como una aproximación, por:

$$
\frac{\mathbf{r}_{0}}{\mathbf{r}}-\mathbf{1}=\frac{\tau}{\tau_{\text {rot }}}
$$

De los parámetros que intervienen en la determinación de la anisotropía, uno de los que resulta menos preciso y que más influencia tiene sobre las curvas de anisotropía, es el tiempo de vida del estado fluorescente $(\tau)$. En la figura 5.5a puede verse el efecto sobre la curva correspondiente a la estructura di-cis con condición de contorno slip, de una variación en $\pm 10 \%$ sobre el valor del tiempo de vida usado para la especie normal (Este es el error asignado a su medida [Velsko] ). Se ve claramente que si bien el apartamiento de las curvas es importante, este no alcanza para entrar en la cota de error de las anisotropías medidas. La precisión con que se determinó la temperatura de la muestra durante las medidas de anisotropía $\left( \pm 0.2^{\circ} \mathrm{C}\right)$, hace que el error que introduce este factor lo podamos despreciar. Asumiendo que en la expresión de la anisotropía, sólo la viscosidad y el tiempo de vida de la fluorescencia dependen de la temperatura, puede verse fácilmente que el error relativo de la anisotropia $(\Delta r / r)$ que resulta, es menor del I \%

No ocurre lo mismo con la forma asignada a cada especie, cuya variación podría producir un cambio notorio en las curvas de anisotropía. Esto se ilustra en la figura $5.5 \mathrm{~b}$, donde ahora se han variado las dimensiones del elipsoide oblado que representa a la estructura di-cis con condición stick, manteniendo el volumen igual al volumen de van der Waals de la molécula. En línea continua se indica la anisotropía correspondiente al modelo antes propuesto para la estructura di-cis; un elipsoide oblado de semiejes 6-6-2.2 $\AA$. En línea punteada se muestra el resultado de aumentar el semieje mayor $(6.3 \AA)$ y disminuir el menor ( $2 \AA$ ), manteniendo constante los demás parámetros. En línea a trazos, la anisotropía correspondiente a un esferoide oblado de dimensiones 5.7-5.7-2.5 $\AA$ 


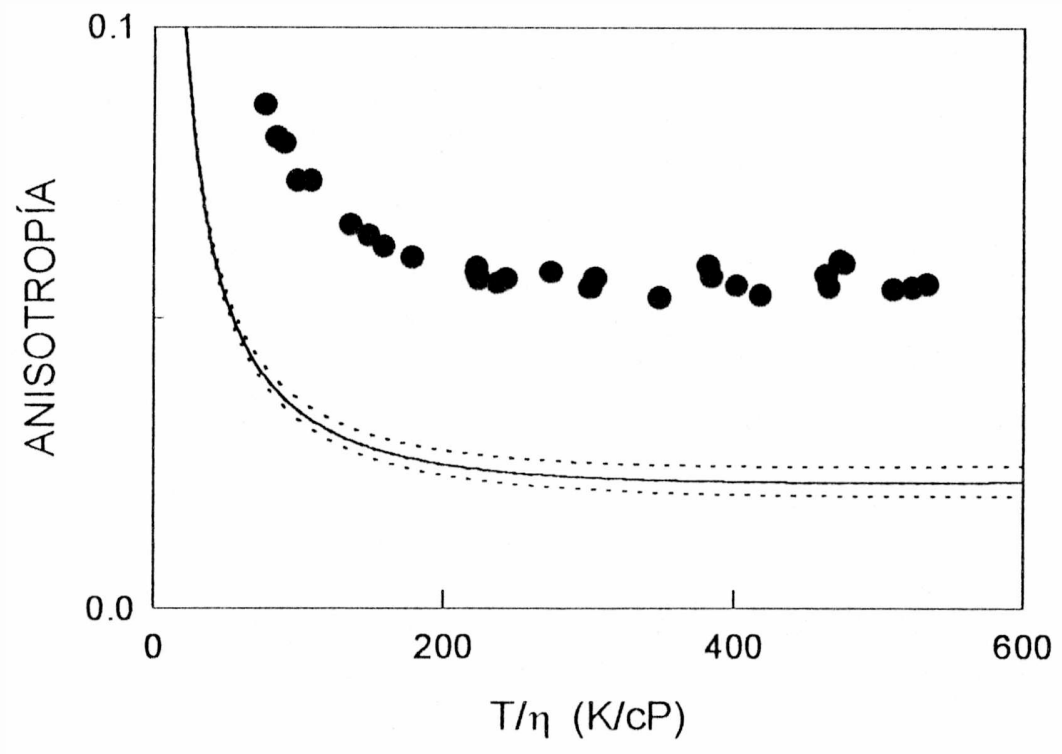

Figura. 5.5a. Ifecto sobre la curva de anisotropia de la fluorescencia, correspondiente a la estructura di-cis con condición de contorno slip (linea continua), de una variación en $\pm 10 \%$ sobre el valor del tiempo de vida del estado fluorescente de la especie normal (linea de puntos).

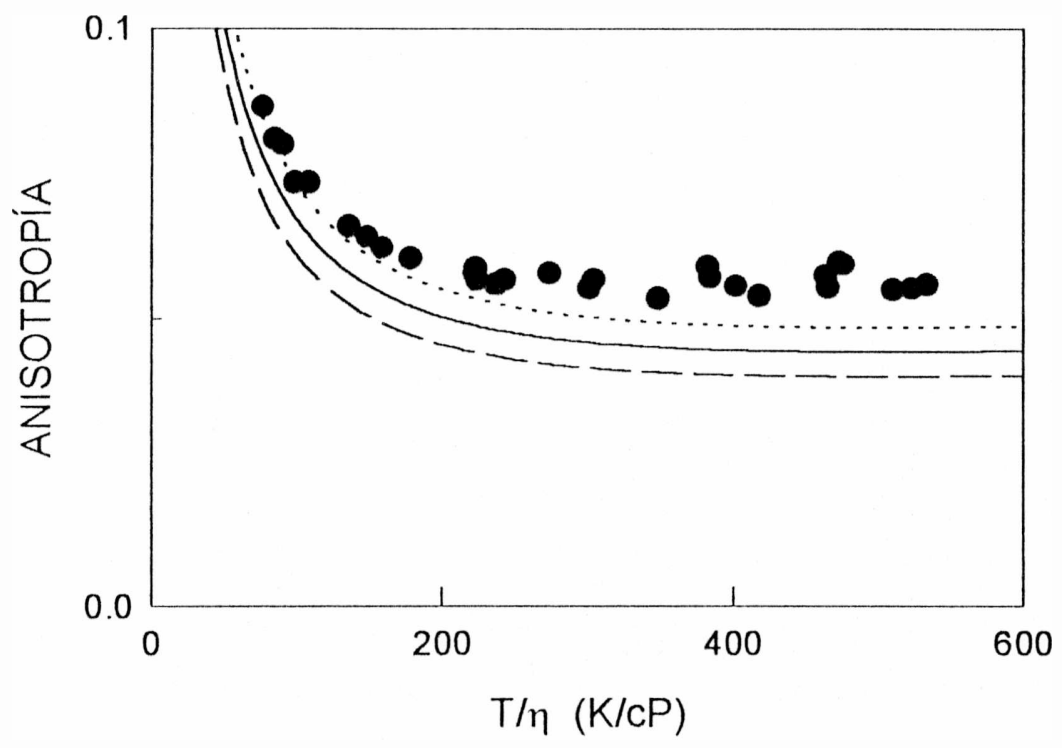

Figura 5.5b. Ffecto de un cambio en las dimensiones del elipsoide oblado que representa a la estructura di-cis con condición stick. La línea continua corresponde al elipsoide con semiejes 6-6-2.2 A. En linea punteada, 6.3-6.3-2 A y en linea a trazos, 5.7-5. 7-2.5 A. 
Volviendo a los resultados mostrados en la figura 5.4, de la primera (5.4a) puede verse que las conformaciones propuestas por Fleming son inconsistentes con nuestros datos experimentales. Sobre la base de sus propios resultados y considerando como válida la condición de contorno stick, él asigna la estructura di-cis (modelo III) a la especie normal del DODCI, y la todo-trans (modelo I) para el fotoisómero. Si bien la curva teórica que representa a la estructura III con condición de contorno stick, parece corresponderse aceptablemente con los datos experimentales para la forma normal; no es posible encontrar en nuestro caso tal correspondencia para el fotoisómero, asumiendo para este último la forma todo-trans. Este desacuerdo se ve reforzado cuando se tiene en cuenta, como ya fuera señalado anteriormente, que hemos subestimado el volumen difusivo de la molécula. Un aumento de este volumen no sólo no permite lograr que se alcance un mejor ajuste, sino que por el contrario, este empeora.

Los valores de anisotropia que hemos medido para la primera especie $(\mathrm{N})$, están en un buen acuerdo con los tiempos de difusión rotacional obtenidos por despolarización de la fluorescencia resuelta en tiempo y por otras técnicas [Fleming \#5I, Shank, Waldeck]. Por su parte, para el fotoisómero los únicos datos disponibles al presente eran aquellos dados por Fleming, los cuales resultan diferentes de los que aquí se presentan. A causa de la evidencia teórica que sugiere para la especie normal de esta cianina la forma todo-trans, intercambiamos los modelos moleculares asignados en primera instancia a los dos isómeros del DODCI. El resultado de este intercambio puede ser observado en la figura (5.4b), donde una vez más vemos que las curvas teóricas de la anisotropía no logran ajustarse a los datos experimentales

Cambiando la condición de contorno hidrodinámica de stick a slip, es posible mejorar el ajuste de los datos experimentales. Cuando se hace esto, los coeficientes de difusión rotacional incrementan sus valores y las curvas teóricas de la anisotropía se desplazan hacia abajo manteniendo sus formas. En la figura (5.4c) se siguió usando la representación anterior de las especies, esto es, modelo I para el isómero normal y modelo III para el fotoisómero, cambiando la condición de contorno por slip. Finalmente, en (5.4d) se muestra el mejor acuerdo, asignando la estructura II al fotoisómero, I para la especie normal, y trabajando con la condición de contorno slip y el volumen de van der Waals para ambas especies.

Es evidente que volúmenes menores que el volumen de van der Waals no pueden ser considerados en la discusión del problema. Pero volúmenes más grandes que aquel podrían ser posibles como consecuencia de interacciones soluto-solvente. Como al aumentar el volumen la anisotropía crece, el ajuste de los puntos experimentales no puede mejorarse bajo la condición de contorno stick. Sin embargo, trabajando con la condición de contorno slip es posible lograr un buen ajuste aumentando el volumen de difusión molecular ( las curvas de la anisotropía suben manteniendo sus formas). En la figura (5.6a) se muestra el mejor ajuste de los puntos experimentales obtenido usando las estructuras todo-trans y di-cis para las 
especies normal y fotoisomérica respectivamente, bajo condición de contorno slip, resultado de incrementar el volumen de las especies en un $49 \%$

En un trabajo reciente [Churio], se ha medido un cambio de volumen durante la fotoisomerización del DODCI en solución. En el mismo se sugiere que el cambio observado se debe al reacomodamiento de moléculas de solvente alrededor de los isómeros con diferentes momentos dipolares, y se determina un volumen asociado con la especie normal mayor que aquel del fotoisómero. Asumiendo como válido el volumen de van der Waals para la forma fotoisómerica y un volumen mayor para la especie normal, $24 \%$ más grande, lo cual representa el cambio de volumen medido en la última réerencia citada, se logra un muy buen acuerdo entre los datos experimentales y las predicciones teóricas; tal como puede ser observado en la figura (5.6b). Esta se corresponde con el detalle de la figura (5.4d), sólo que ahora se ha modificado el volumen de la forma normal.

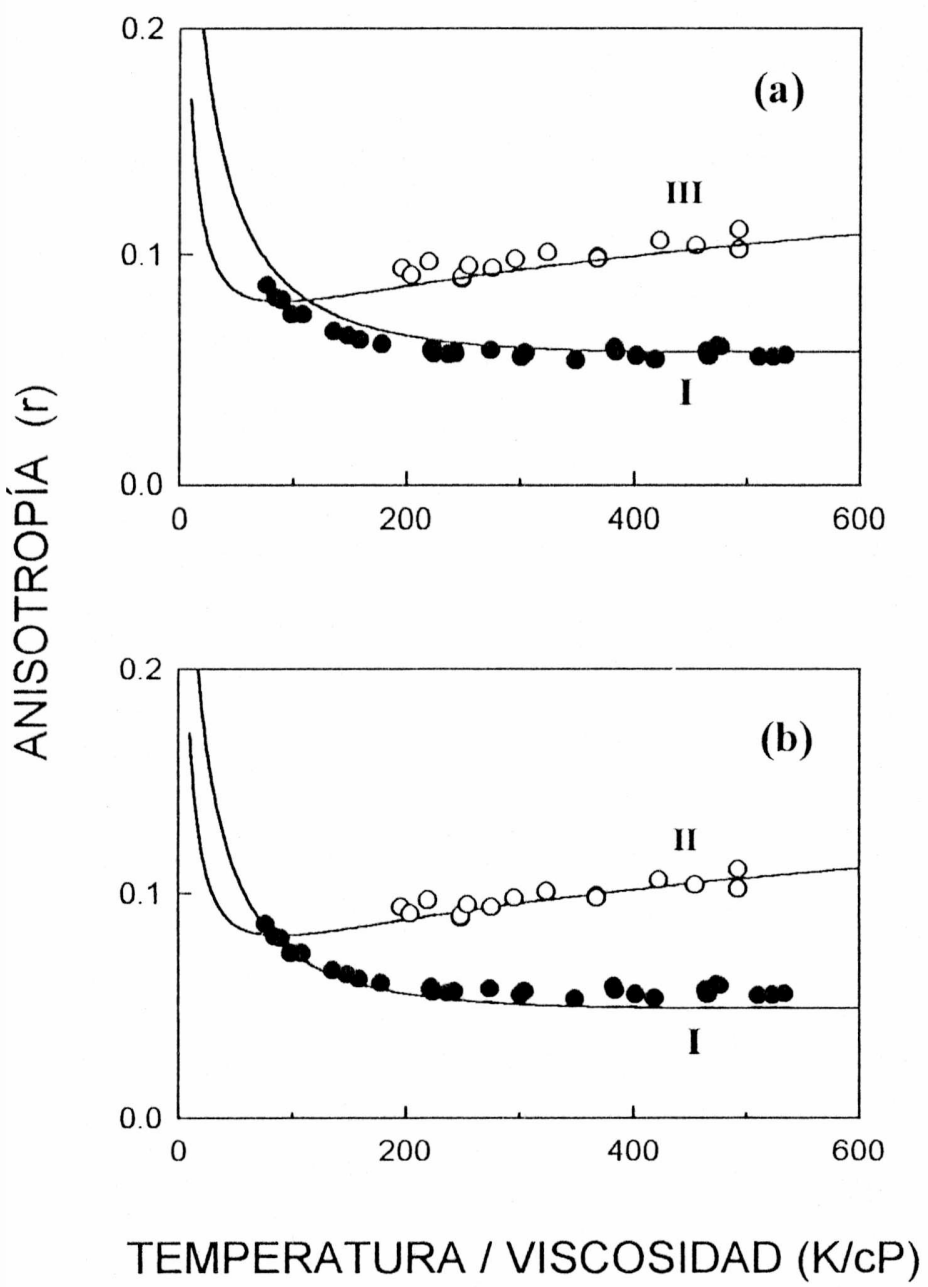

Figura 5.6 


\subsection{POLARIZACIÓN SATURADA.}

Con la idea de obtener información de las estructuras fotoisoméricas sin necesidad de separar los espectros de fluorescencia de ambas especies, se estudió la posibilidad de lograr esa información analizando la anisotropia en función de la fluencia de excitación. Tal como fuera señalado en el capítulo anterior, cuando esta fluencia es muy baja, la cantidad de fotoisómero que se forma es despreciable, entonces la anisotropía que se mide corresponde a la especie normal. A medida que la fluencia de excitación aumenta, crece la participación del isómero generado por la excitación luminosa, cambiando entonces el valor de la anisotropía. Luego, en saturación, cuando la potencia de excitación es tal que la población de fotoisómero que se forma es máxima, el cambio en la polarización alcanzará también un máximo, y a partir de este valor sería posible inferir la estructura de la segunda especie. Aún en aquellos casos en que los espectros se encuentren completamente superpuestos, debería medirse un cambio en la anisotropía de la fluorescencia, a causa de la contribución de la segunda especie con una estructura diferente a la de la primera y por ende una difusión rotacional distinta. Podría entonces abordarse el estudio de la anisotropía de las especies transientes de aquellos colorantes que presentan espectros de fluorescencia muy superpuestos con los de sus especies estables, sin necesidad de contar con las emisiones de una y otra especie por separado. Sería esta una posible vía de lograr información acerca de la estructura de sus fotoisómeros y así aumentar el conocimiento sobre el comportamiento de las especies fotoisoméricas de estas moléculas en solución

Este método se ha aplicado al estudio de la anisotropía del DODCI, con el fin de confirmar los resultados ya obtenidos a partir de los espectros de fluorescencia resueltos de ambas especies isoméricas de este colorante, y asi probar su utilidad. En lo que sigue se detallan las ecuaciones utilizadas y se analizan los resultados obtenidos.

\section{- teoría.}

Cuando las dos especies están fluoresciendo, en la expresión de la anisotropía aparecerá la contribución de ambas. Así, llamando $\mathbf{I}_{\mathrm{N}}^{\mathrm{p}, \mathrm{s}}$ a las componentes de la especie normal con polarización paralela y perpendicular a la de excitación, e $\mathbf{I}_{\mathbf{P}}^{\mathbf{p}, \mathbf{s}}$ a las respectivas componentes del fotoisómero, se tendrá:

$$
\mathbf{r}=\frac{\left(\mathbf{I}_{\mathrm{N}}^{\mathrm{p}}+\mathbf{I}_{\mathrm{P}}^{\mathrm{p}}\right)-\left(\mathbf{I}_{\mathrm{N}}^{\mathbf{s}}+\mathbf{I}_{\mathrm{P}}^{\mathrm{s}}\right)}{\left(\mathbf{I}_{\mathrm{N}}^{\mathrm{p}}+\mathbf{I}_{\mathrm{P}}^{\mathbf{p}}\right)+2\left(\mathbf{I}_{\mathrm{N}}^{\mathbf{s}}+\mathbf{I}_{\mathrm{P}}^{\mathrm{s}}\right)}
$$


Operando algebraicamente esta anisotropía puede expresarse en términos de aquellas correspondientes a las especies normal y fotoisomérica del colorante.

$$
\mathbf{r}=\frac{\mathbf{r}_{\mathrm{N}}}{1+\Phi^{-1}}+\frac{\mathbf{r}_{\mathrm{p}}}{1+\Phi}
$$

donde $\Phi=\frac{I_{\mathrm{N}}^{\mathrm{p}}+2 \mathbf{I}_{\mathrm{N}}^{\mathrm{s}}}{\mathbf{I}_{\mathrm{P}}^{\mathrm{p}}+2 \mathbf{I}_{\mathrm{P}}^{\mathrm{s}}}$

$\Phi$ es el cociente entre la intensidad total emitida por la especie normal y aquella emitida por el fotoisómero. Estas intensidades pueden ser expresadas en términos de las intensidades absorbidas y las eficiencias de fluorescencia de una y otra especie. De esta manera podemos volver a escribir:

$$
\Phi=\frac{\mathbf{I}_{\mathrm{a}}^{\mathrm{N}} \cdot \phi_{\mathrm{r}}^{\mathrm{N}}}{\mathbf{I}_{\mathrm{a}}^{\mathrm{P}} \cdot \phi_{\mathrm{r}}^{\mathrm{P}}}
$$

Asumiendo que la intensidad absorbida es la diferencia entre la intensidad incidente sobre la celda que contiene la muestra y la intensidad transmitida, y usando la ley de Lambert Beer para relacionar estas dos últimas, resulta para la intensidad absorbida por cada especie la siguiente expresión:

$$
\mathbf{I}_{\mathrm{a}}^{\mathrm{i}}=\mathbf{I} \cdot\left(1-10^{-\Lambda_{1}}\right) \quad \mathrm{i}=\mathbf{N}, \mathbf{P}
$$

donde $\mathbf{I}$ es la intensidad incidente y $\boldsymbol{A}_{\mathbf{i}}$ la absorbancia de la especie $\mathbf{i}$

Ahora bien, si la absorbancia es pequeña, la intensidad absorbida será proporcional a ésta. Suponiendo que las absorbancias de ambas especies son lo suficientemente pequeñas como para que sea válida la aproximación, el factor $\Phi$ podrá volver a escribirse como sigue:

$$
\Phi=\frac{\mathbf{A}_{\mathrm{N}}}{\mathbf{A}_{\mathrm{P}}} \cdot \mathbf{f}^{-1}=\frac{\sigma_{\mathrm{N}} \cdot \mathbf{N}_{0}}{\sigma_{\mathrm{P}} \cdot \mathbf{P}_{0}} \cdot \frac{1}{\mathbf{f}}
$$

donde $\sigma_{\mathbf{i}}$ es la sección eficaz de absorción de la especie $\mathbf{i}$, y $\mathbf{N}_{\mathbf{0}}$ y $\mathbf{P}_{0}$ son las poblaciones en el estado fundamental de la especie normal y fotoisómero, respectivamente, y $\mathbf{f}$ es el cociente de las eficiencias cuánticas de fluorescencia 
Hay un elemento cuyo efecto no ha sido tenido en cuenta hasta aqui. En el sistema de recolección de la luz emitida por la muestra, se colocó un filtro para evitar la luz dispersada del láser de excitación. Este filtro recorta las emisiones de una y otra especie en forma diferente. De manera que el cociente de las intensidades emitidas por las especies deberá incluir un factor $(\gamma)$ que represente la fracción del espectro de fluorescencia de cada especie que pasa a través del filtro. Entonces,

$$
\Phi=\frac{N_{0}}{P_{0}} \cdot \frac{1}{B . f} \cdot \gamma
$$

$\mathbf{N}_{0}$ y $\mathbf{P}_{0}$ se relacionan con la fluencia de excitación (ver sección 4.2.1) por la siguiente igualdad:

$$
\frac{\mathbf{N}_{0}}{P_{0}}=\mathbf{p} \cdot \mathbf{B}+(1+\mathbf{p} \cdot \mathbf{B}) \cdot \frac{\mathbf{a}_{\mathrm{s}}}{\mathrm{a}}
$$

Finalmente, la expresión que resulta para $\Phi$ es como sigue:

$$
\Phi=\frac{\gamma}{\text { B.f }} \cdot\left[\mathbf{p} \cdot \mathbf{B}+(\mathbf{1}+\mathbf{p} \cdot \mathbf{B}) \frac{\mathbf{a}_{\mathbf{s}}}{\mathbf{a}}\right]
$$

Cuando la fluencia de excitación es mucho mayor que la correspondiente a saturación, el factor $\Phi$ se reduce a :

$$
\Phi=\gamma \cdot \frac{\mathbf{p}}{\mathbf{f}}
$$

resultando ahora independiente del cociente de secciones eficaces de absorción de una y otra especie (B)

\section{- desarRollo de la experiencia y resultados}

La polarización del DODCI en función de la fluencia de excitación, se midió en la forma que se detallara oportunamente cuando se determinó la anisotropía de su especie normal. Las medidas se realizaron a temperatura ambiente, excitando la muestra en $584 \mathrm{~nm}$. En esta oportunidad, se utilizó un filtro Schott RG 630 detrás del analizador para evitar la 
luz dispersada del láser de excitación, y a la vez dar una mayor participación a la fluorescencia del fotoisómero respecto de la especie normal. En la figura 5.7, sobre los espectros normalizados de absorción y emisión de ambas especies, se indican la longitud de onda de excitación y el recorte de los espectros de fluorescencia ( b' y d' ) por acción del filtro utilizado

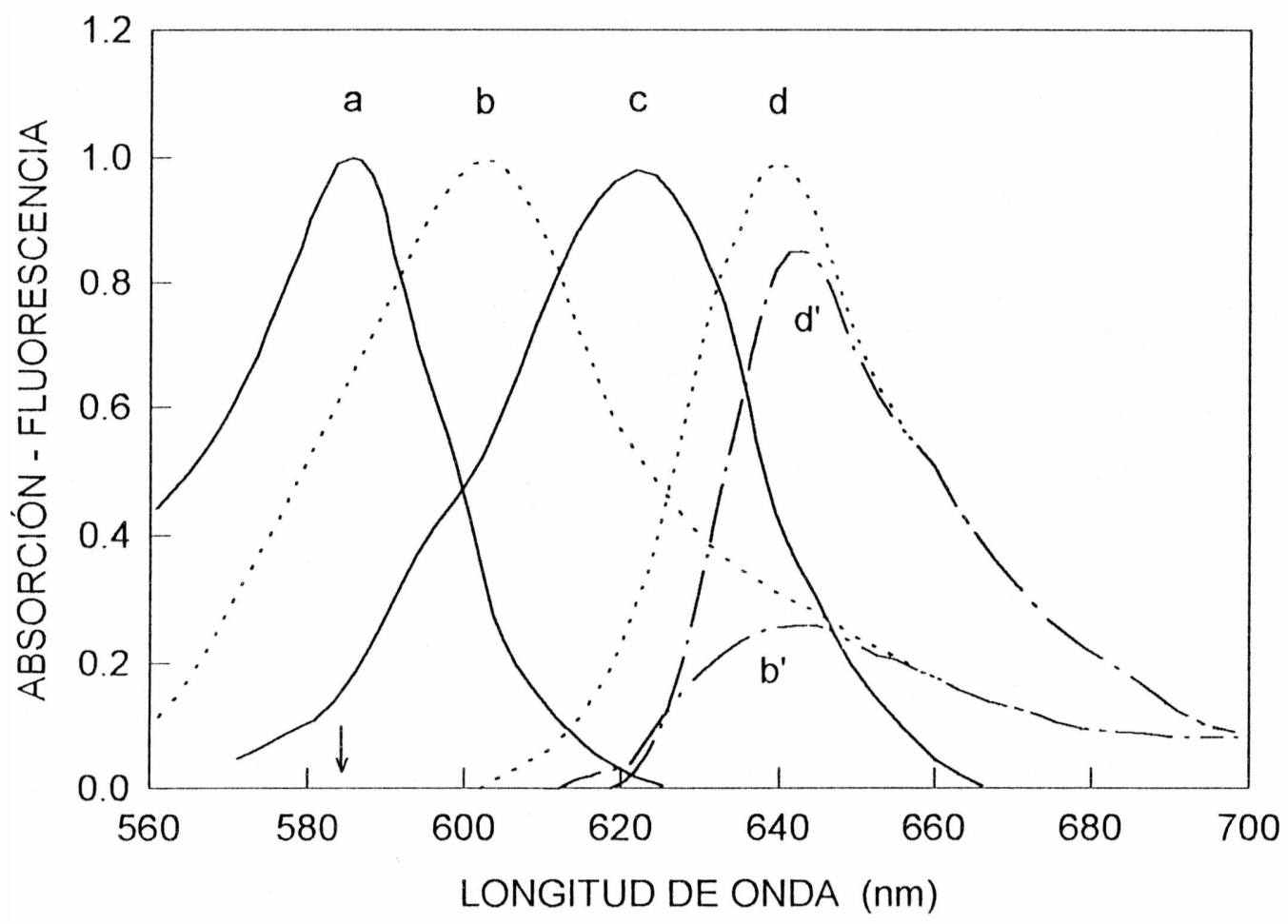

a : absorción de la especie normal. b : fluorescencia de la especie normal.

$\mathrm{c}$ : absorción del fotoisómero. d : fluorescencia del fotoisómero.

Figura 5.7

La fluencia de excitación se varió cuatro órdenes de magnitud, cambiando la potencia del láser de excitación y/o enfocando el haz sobre la celỏa con lentes de diferente distancia focal ( 6,7 y $40 \mathrm{~cm}$ ). La fuencia de excitación en la condición de saturación resultó ser $\mathrm{a}_{\mathrm{s}} \cong$ $10^{4} \mathrm{~s}^{-1}$. 
En la figura 5.8 puede verse el resultado de las medidas experimentales, junto con dos curvas que simulan el comportamiento esperado acorde a la ecuación (5.3). En línea punteada se indica el resultado de usar un valor de $\mathrm{B}=0.15$ en acuerdo con la figura 5.7. El espectro de absorción de $\mathbf{P}$ que allí aparece es el que se determinara en el trabajo de Bilmes [1988]. Si en lugar de este valor de B se usa 0.3 , el ajuste mejora ( línea continua ). $\mathrm{B}=0.3$ corresponde a una distribución del espectro de absorción de $\mathrm{P}$ que se asemeja más a la de la especie normal

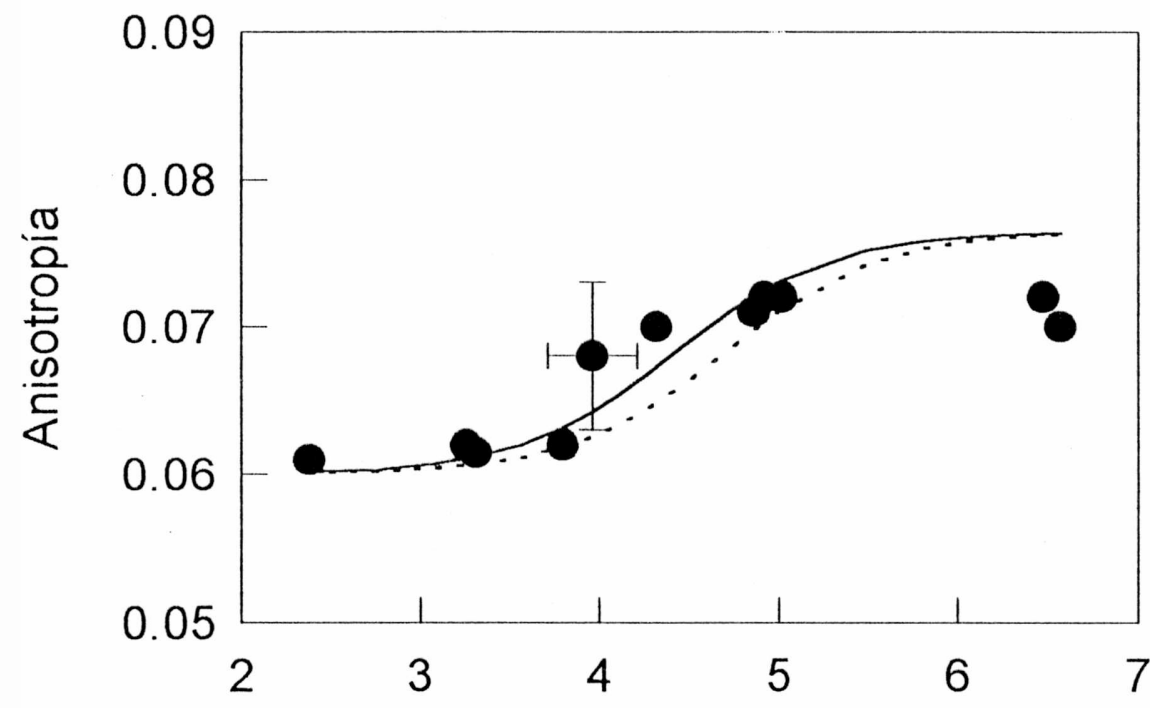

Log. de la fluencia de excitación (1/s)

Figura 5.8 .

Como valores del cociente de eficiencias cuánticas de isomerización (p) y de eficiencias cuánticas de fluorescencia (f), se usaron en el ajuste los valores hallados oportunamente (Cap. 4), $\mathrm{p}=0.36$ y $\mathrm{f}=0.25$. Los valores de anisotropía usados fueron, $r_{N}=0.06$ y $\quad r_{p}=0.085$. El valor de $\gamma$ se midió a partir del recorte de los espectros de fluorescecia de ambas especies ( Fig. 5.7), resultando igual a 0.36

Cuando la fluencia de excitación es mucho mayor que la fluencia de saturación, la anisotropía medida se incrementa aproximadamente en un veinte por ciento respecto de aquella que resulta con baja fluencia y que por ende corresponde a la especie normal. Dentro de los errores experimentales y teniendo en cuenta la incerteza con que se conocen los 
parámetros que se usaron en el ajuste, este incremento nos permite decir que la anisotropía del fotoisómero no es mayor que aquella medida utilizando la técnica de doble excitación y sí es mayor que la anisotropía correspondiente a la especie normal de esta molécula. Si bien la polarización saturada por si sola no basta para una determinación precisa de la anisotropia de las especies fotoisoméricas, al menos en este caso, permitió que se logrará obtener un valor acotado de la misma. La aplicación de este método a las restantes cianinas estudiadas no permite obtener información de sus especies fotoisoméricas, en principio por el valor bajo de sus eficiencias de fluorescencia. 


\subsection{ESTUDIO DE LA DIFUSIÓN ROTACIONAL DE LAS ESPECIES NORMALES.}

Tal como ya fuera señalado, la imposibilidad de acceder con la técnica de polarización de la luminiscencia a las especies fotoisoméricas de las restantes cianinas aquí estudiadas, es una consecuencia de las características espectroscópicas de las mismas. Estas características limitan el estudio de la anisotropia de la fluorescencia a sus especies normales ". A partir de los resultados de polarización obtenidos fue posible analizar en conjunto el comportamiento de estas especies, que presentan diferencias en su estructura como ser el largo de la cadena polimetínica o el heteroátomo en los grupos terminales.

El desarrollo experimental es como se indicó para el estudio del DODCI. Todas las cianinas fueron disueltas en etanol. En cada caso, la excitación y el filtro usado para eliminar la luz dispersada del láser, resultó adecuada a las características espectroscópicas de las cianinas estudiadas. Así, para DTDCI se usó un láser de He-Ne y el filtro de color empleado fue un Schott RG-695. Las muestras fueron excitadas en forma continua con baja fluencia, aproximadamente $0.1 \mathrm{~W} / \mathrm{cm}^{2}$

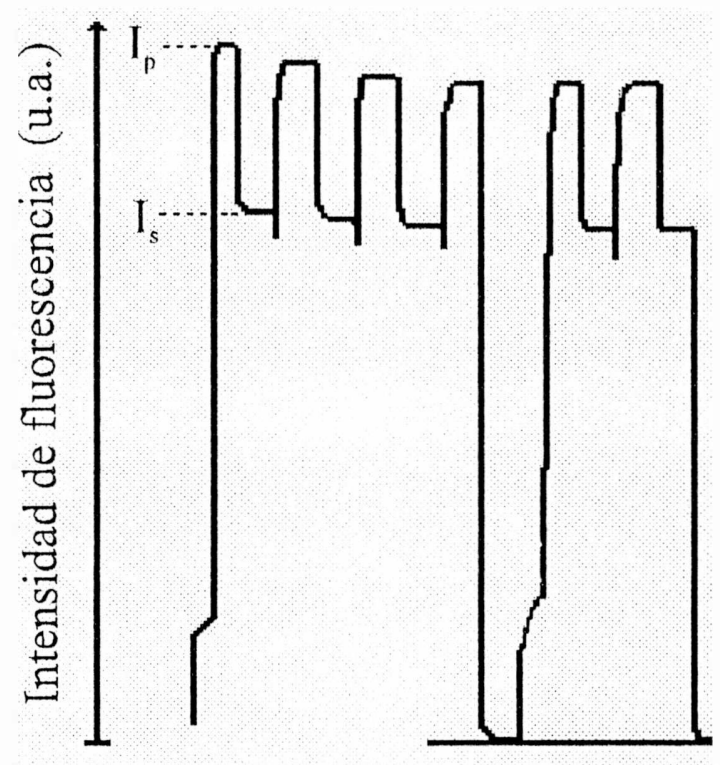

Figura 5.9

Para DTDCI a temperatura ambiente, la figura 5.9 muestra el resultado obtenido, sin corregir por la respuesta del equipo experimental, al medir alternativamente las intensidades

"La anisotropía de la fluorescencia en estado estacionario de las especies normales de las cianinas aquí estudiadas, ha sido medida recientemente en diferentes alcoholes [Levilus], estando los resultados mostrados en etanol en un buen acuerdo con los aqui obtenidos. 
de fluorescencia con polarización paralela y perpendicular a la dirección de polarización de la excitación.

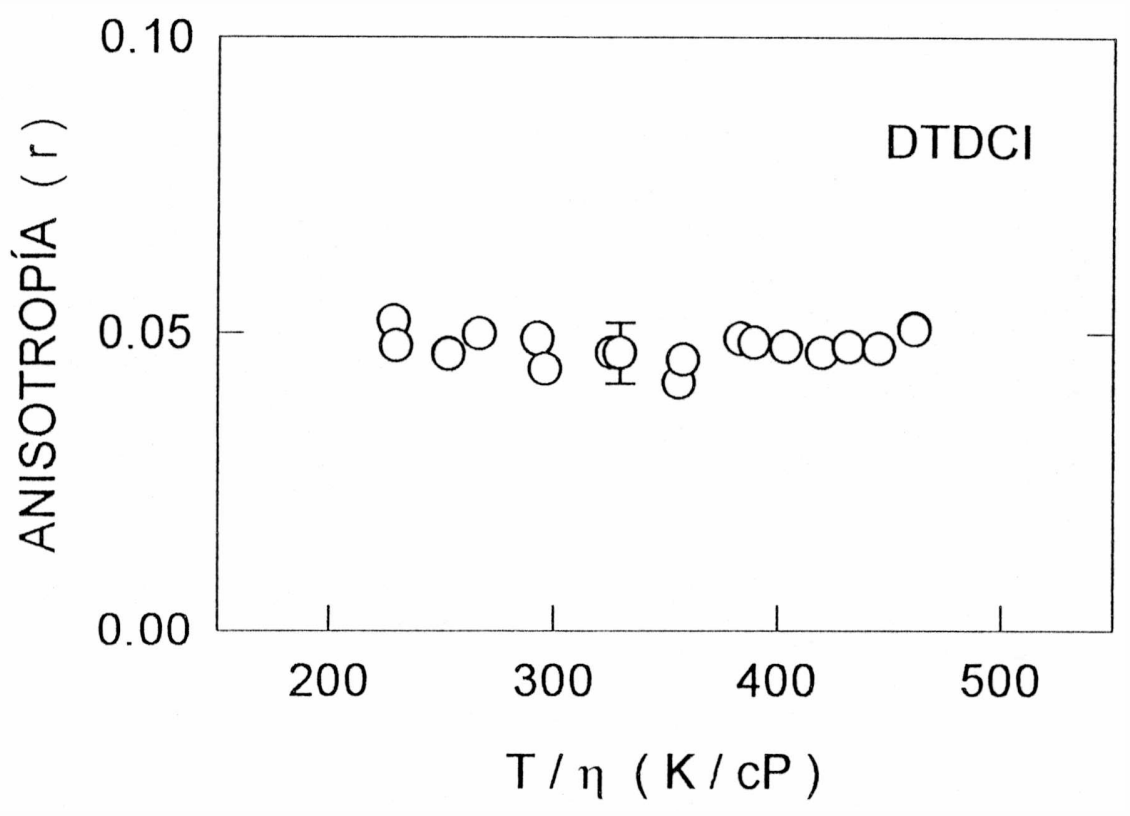

Figura 5.10

En la figura 5.10 pueden verse distintos valores de la anisotropía de la fluorescencia del DTDCI, obtenidos variando la temperatura de la solución, como función del cociente temperatura / viscosidad. Todas las medidas tienen un valor próximo entre sí, que está dentro del error experimental. Si bien al aumentar la temperatura el tiempo de difusión rotacional de la molécula disminuye, también disminuye el tiempo de vida de su fluorescencia, compensándose los dos efectos, lo cual resulta en un valor prácticamente contante de su anisotropía dentro del rango medido.

Atendiendo a la discusión de la anisotropía en el caso del DODCI, y en acuerdo con análisis teóricos que apoyan esta hipótesis, se asumirá que las especies más estables de las cianinas estudiadas en este trabajo, adoptan bajo condiciones normales en solución una conformación todo-trans. Luego, la forma de las moléculas será simulada con elipsoides asimétricos, cuya forma y dimensiones son una vez más consistentes con estas estructuras propuestas y con el volumen de van der Waals de las moléculas. Para DTDCI se usó el mismo elipsoide que representó a la especie todo-trans del DODCI. En el caso de las cianinas de cadena más corta se modificó sólo el semieje mayor del elipsoide. De acuerdo con la longitud de un eslabón de la cadena, esto es un átomo de carbono ligado al resto de la 
molécula por un enlace simple y uno doble formando cierto ángulo entre sí, se tomó para este semieje el valor $\mathbf{a}=9 \AA$.

En la figura 5.11 se representan las formas todo-trans de estas moléculas junto con los elipsoides que las simulan. Arriba se puede apreciar una vista en el plano molecular, y a continuación una vista lateral de las mismas, resultado de rotar alrededor del eje mayor de los elipsoides a un plano perpendicular
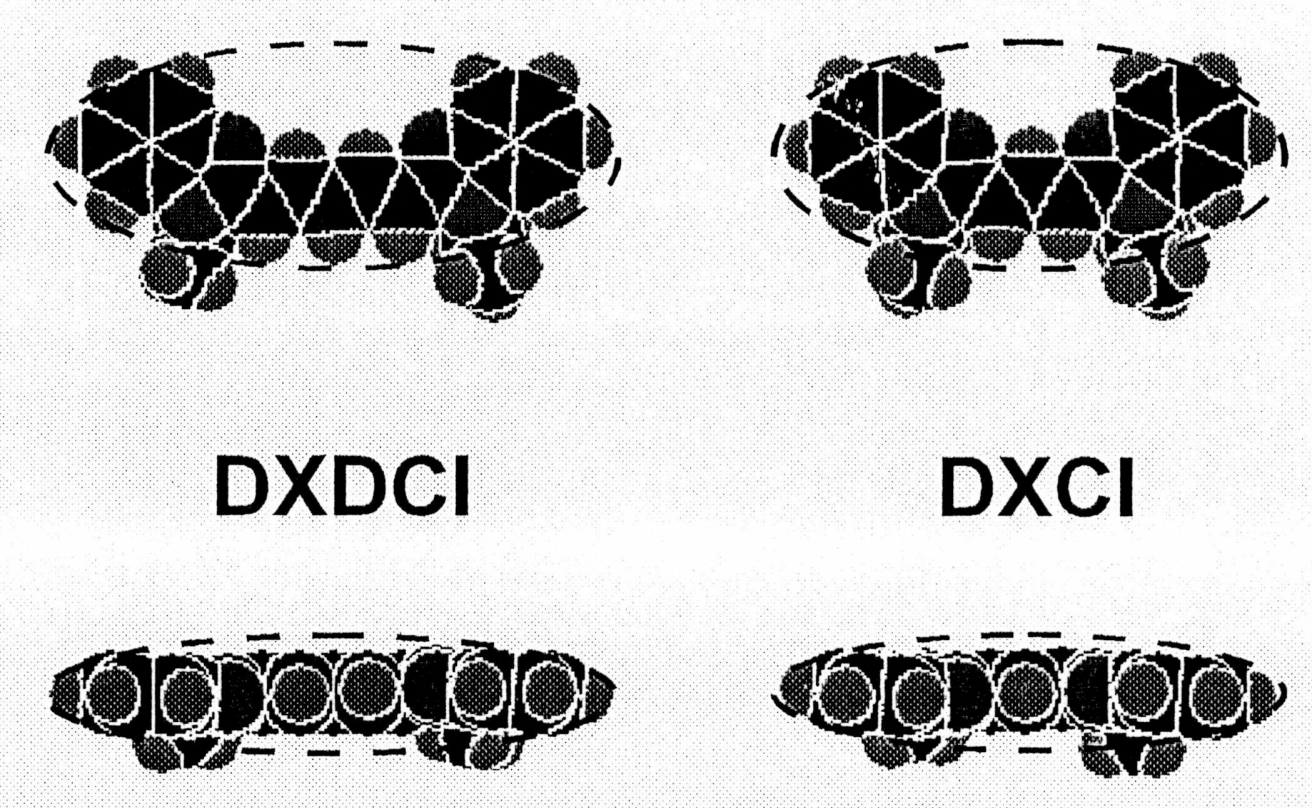

Figura 5.11. Vista de la estructura todo-trans de las moléculas estudiadas. X puede estar representando al azufre como heteroátomo (DTDCI, DTCI) o al oxígeno (DODCI, DOCI).

Se asume que los momentos de transición en absorción y emisión de estas cianinas, son colineales $\left(r_{o}=0.4\right)$. En la tabla 5.2 se indican las dimensiones de los dos elipsoides usados en el modelado de las estructuras moleculares y los tiempos de difusión rotacional calculados para ambas condiciones de contorno hidrodinámicas, siguiendo el procedimiento que fuera indicado en el capítulo 3.

En cualquier caso, el decaimiento de la anisotropía calculado resulta prácticamente monoexponencial. Esto era de esperar habiéndose asumido para las especies normales de las cianinas estudiadas la forma todo-trans. La expresión de la anisotropía correspondiente a los elipsoides asimétricos con que se modelan estas moléculas, podrá entonces ser escrita en la 


\begin{tabular}{||c|c|c|c|c|c||}
\hline semiejes $(\boldsymbol{A})$ & $\mathbf{A}_{\mathbf{1}}$ & $\mathbf{A}_{\mathbf{2}}$ & $\mathbf{a}_{\mathbf{1}}\left(\mathbf{n s}^{\mathbf{- 1}}\right)$ & $\left.\mathbf{a}_{\mathbf{2}} \mathbf{( n s}^{\mathbf{1}}\right)$ & c.c.h. \\
\hline \hline $\begin{array}{c}10-4-2 \\
(\mathrm{DXDCI})\end{array}$ & 0.0001 & 0.3999 & 8.56 & 3.41 & stick \\
\cline { 2 - 6 } & 0.002 & 0.398 & 30.5 & 7.5 & slip \\
\hline $\begin{array}{c}9-4-2 \\
(\mathrm{DXCI})\end{array}$ & 0.0001 & 0.3999 & 9.58 & 4.31 & stick \\
\cline { 2 - 6 } & 0.005 & 0.395 & 38.58 & 10.96 & slip \\
\hline
\end{tabular}

Los coeficientes fueron calculados en las condiciones que se indican para la tabla 5.1.

forma ya indicada anteriormente,

$$
\frac{r_{0}}{r}-1=\frac{\tau}{\tau_{\text {rot }}}
$$

En esta ecuación el tiempo de difusión rotacional puede ser expresado como,

$$
\tau_{\mathrm{rot}}=\frac{\eta \mathbf{V}}{\mathrm{kT}} \cdot \frac{\mathbf{F}}{\mathrm{S}}
$$

donde $\mathbf{F}$ es un factor de fricción, que tiene en cuenta la condición de contorno hidrodinámica que se aplica, y $\mathbf{S}$ un factor de forma asociado a la simetría molecular. $\mathbf{F}$ puede tomar cualquier valor entre cero y uno, y es igual a uno con la condición de contorno stick. Para elipsoides simétricos $\mathbf{S}$ depende sólo del cociente entre los semiejes mayor y menor. El conjunto V.F/S suele definirse como el volumen molecular efectivo.

Si la difusión rotacional de la molécula de soluto en solución está gobernada sólo por la fricción mecánica, no intervienen por ejemplo efectos dieléctricos, el tiempo de difusión rotacional variará linealmente con el cociente viscosidad / temperatura, con una pendiente que será independiente del solvente.

La expresión correspondiente a la anisotropía de la fluorescencia (5.12) puede volver a escribirse como sigue:

$$
\frac{\tau}{\frac{r_{0}}{r}-1}=C \cdot \frac{\eta}{T}
$$


donde $\mathbf{C}$ es una constante. Al graficar el primer miembro de esta ecuación en función del cociente viscosidad/temperatura, se debería obtener una recta si se cumplen las hipótesis antes señaladas. En la figura 5.12 se muestra el resultado de adoptar esta representación para la especie normal del DODCI. Las líneas rectas que aparecen en el gráfico representan las predicciones teóricas stick-slip, considerando un volumen efectivo de rotación mayor $(\sim 25 \%)$ que el volumen de van der Waals de la molécula.

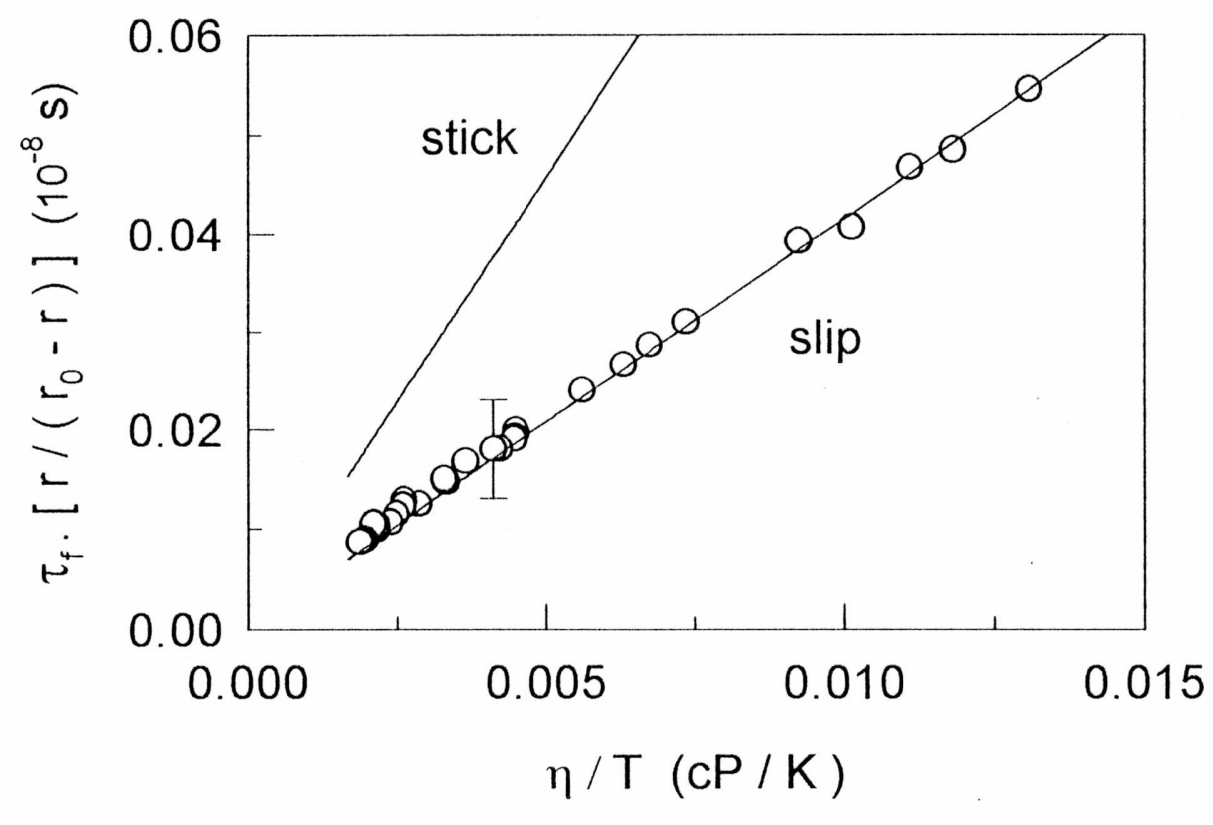

Figura 5.12. Tiempos de difusión rotacional de la especie normal del DODCI.

Volviendo al caso del DTDCI, la representación de su tiempo de difusión rotacional en función de cociente viscosidad/temperatura se muestra en la figura 5.13a. Con líneas rectas se indican las predicciones de ambos modelos hidrodinámicos. El elipsoide usado para simular la molécula, es el mismo que se empleó en el caso del DODCI. Existe una diferencia de volumen entre la molécula del colorante DTDCI y la de DODCI, a causa de la presencia ya sea de azufre u oxígeno en éstas. El DTDCI resulta ser aproximadamente $23 \AA^{3}$ más grande que el DODCI cuando se evalúa el incremento por volúmenes de van der Waals [Edward, Bondi]. Si esta diferencia de volumen es sumada al volumen del elipsoide con que se modeló la molécula, el resultado sobre el comportamiento hidrodinámico de la misma es un aumento en la pendiente de las rectas, que puede ser apreciado en linea de puntos en la 


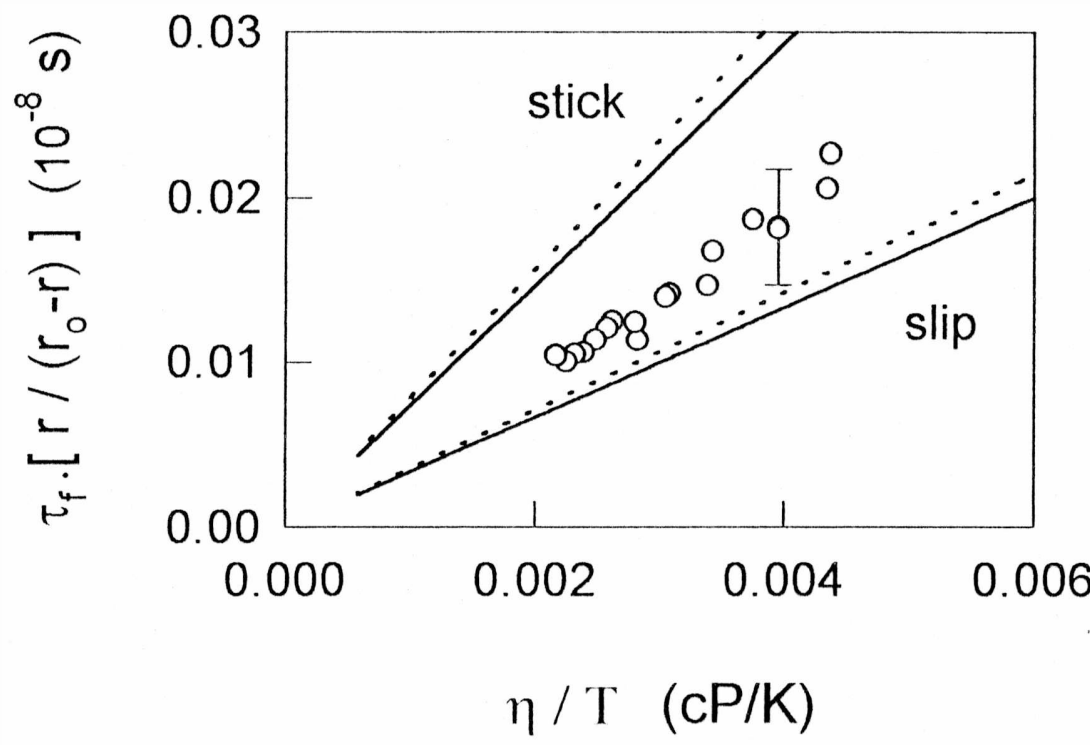

Figura 5.13a. Tiempos de difusion rotacional del DTDCI. En linea de puntos el resultado de aumentar el volumen de la molécula en $23 A^{3}$.

misma figura 5.13a. En 5.13b lo que se ha hecho, de nuevo como se hizo en el caso del DODCI, es aumentar el volumen de la molécula hasta alcanzar un volumen efectivo $25 \%$ mayor que el del elipsoide usado en la simulación de la forma molecular. El resultado que se observa es similar al que se encontrara para DODCI, en iguales condiciones.

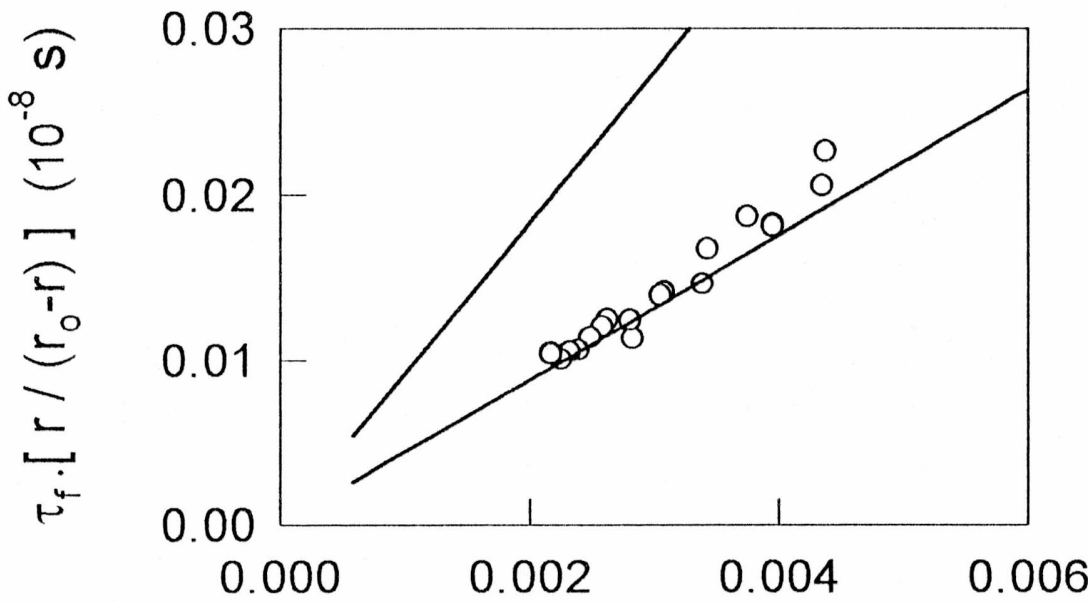

$\eta / \mathrm{T} \quad(\mathrm{cP} / \mathrm{K})$

Figura 5.136. DTDCI con un aumento del 25\% de su volumen de van der Waals. 
Para las cianinas de cadena corta ( DTCI y DOCI), la anisotropía obtenida resultó ser similar para las dos especies, y ésta a su vez mayor que la correspondiente a las dos cianinas de cadena más larga. Ambos resultados eran de esperar en virtud del comportamiento similar observado entre DODCI y DTDCI, y del hecho que los tiempos de vida de las especies excitadas de las cianinas de cadena más corta son menores que los de las otras cianinas.

En la figura 5.14 pueden verse los valores de la anisotropía obtenidos para DOCI y DTCI, en términos del cociente temperatura/viscosidad. Para cada molécula, de nuevo se
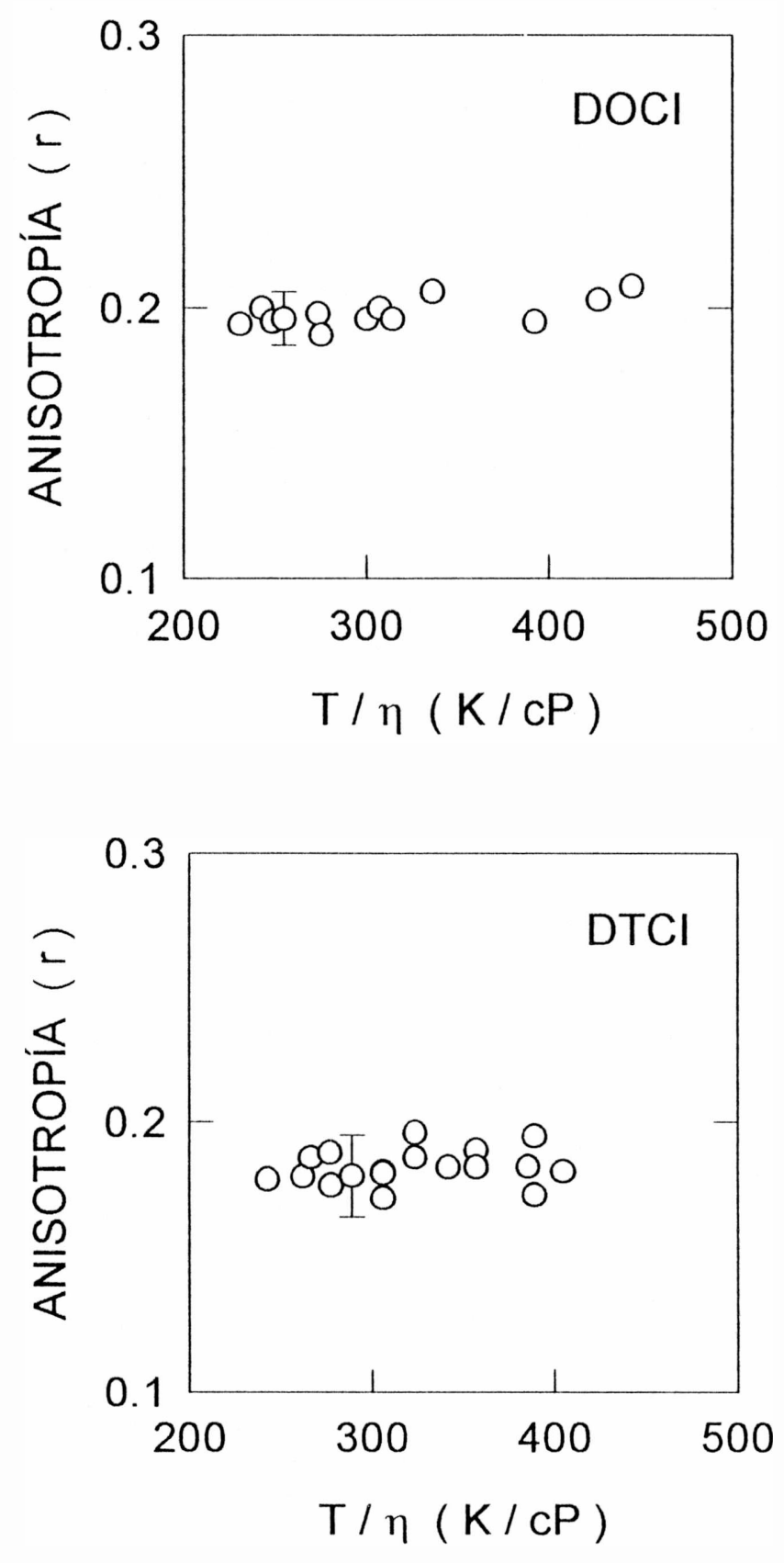

Figura 5.14 
observa que todas las medidas tienen un valor próximo entre sí. La mayor dispersión de los puntos en el caso del DTCI, tiene que ver con la estabilidad en potencia del láser de colorante utilizado. Si el láser no es estable la diferencia en la intensidad de fluorescencia puede no sólo depender de la polarización de observación, sino también de un cambio en la fluencia de excitación.

EI DTCI se excitó en $\lambda_{\text {exc. }}=553 \mathrm{~nm}$, y el filtro utilizado para eliminar la luz dispersada del láser de excitación fue un C.S. 2-73 (Corning Glass Works). En el caso particular del DOCI, se excitó con el láser de argón en $488 \mathrm{~nm}$ y se usó un filtro Schott OG-515. Al igual que se hiciera con el DODCI, con esta última cianina (DOCI) se midió la anisotropía cambiando la fluencia de excitación. Más precisamente, se varió la potencia de excitación en dos órdenes de magnitud (1-100 mW), viéndose sólo cambios de la anisotropía dentro del error experimental de estas medidas.

En la figura 5.15 se han representado los tiempos de difusión rotacional del DTCI, calculados a partir de la anisotropía medida en función de la temperatura de la solución, en términos del cociente $\eta / T$. Para este cálculo se requiere conocer la dependencia del tiempo de vida de la fluorescencia con la temperatura, dependencia que se indica en el apéndice $C$. En línea a trazos se muestran las predicciones de los modelos hidrodinámicos, acorde a los datos de la tabla 5.2. En línea continua se indica el resultado de usar aquellos mismos modelos cambiando sólo el volumen efectivo de la molécula, con un incremento del $25 \%$ sobre el volumen de van der Waals de la misma. Finalmente en línea de puntos, y sólo para el modelo slip, el resultado de sumar $23 \AA^{3}$ más al volumen molecular, como se hiciera en el caso del DTDCI.

De la figura 5.15 puede advertirse a simple vista que una regresión lineal de los puntos tiene una pendiente que es claramente diferente de aquella correspondiente a la recta teórica que resulta del uso del modelo hidrodinámico slip, aún con un volumen molecular efectivo mayor que el volumen de van der Waals. Esta diferencia puede tener su origen en la elección que se hiciera de los parámetros de Arrhenius para el ajuste de la dependencia del tiempo de vida de la fluorescencia de esta molécula con la temperatura.

Con el fin de poder apreciar el efecto de un cambio en los valores de los parámetros que relacionan el tiempo de vida $\left(\tau_{f}\right)$ con la temperatura, se incluye la figura 5.16. En esta se han vuelto a representar los tiempos de difusión rotacional en función del cociente viscosidad I temperatura (círculos), junto con la recta teórica que mejor se aproxima (aquella en línea de puntos), y se ha sumado a esta representación los tiempos de difusión rotacional $(\Lambda)$ que resultan de cambiar el conjunto de parámetros usados para establecer la dependencia $\tau_{1}(\mathrm{~T})$. En cada caso se muestra con línea continua la regresión lineal.

Los viejos parámetros, aquellos que se usaron en la representación de la Fig. 5.15, se indican entre paréntesis a continuación de la figura 5.16 (ver también apéndice C). El nuevo conjunto de parámetros se señala con un asterisco. 


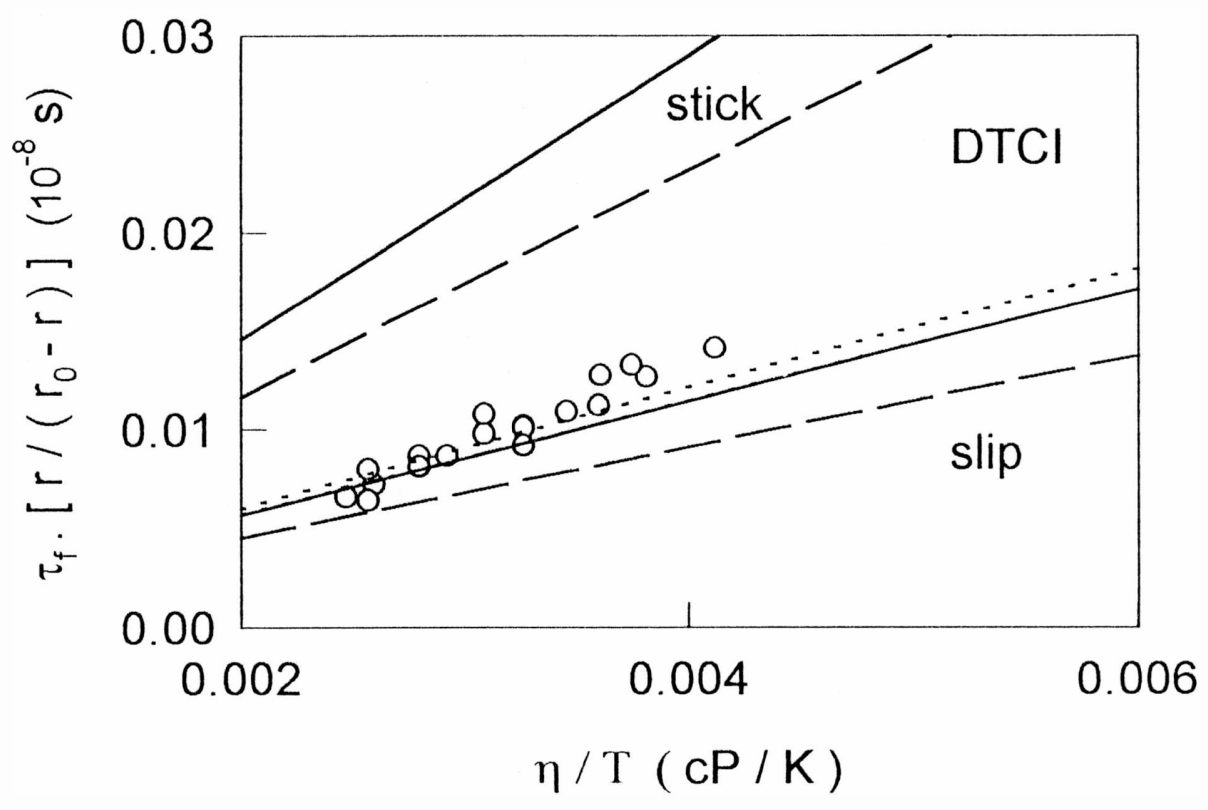

Figura 5.15

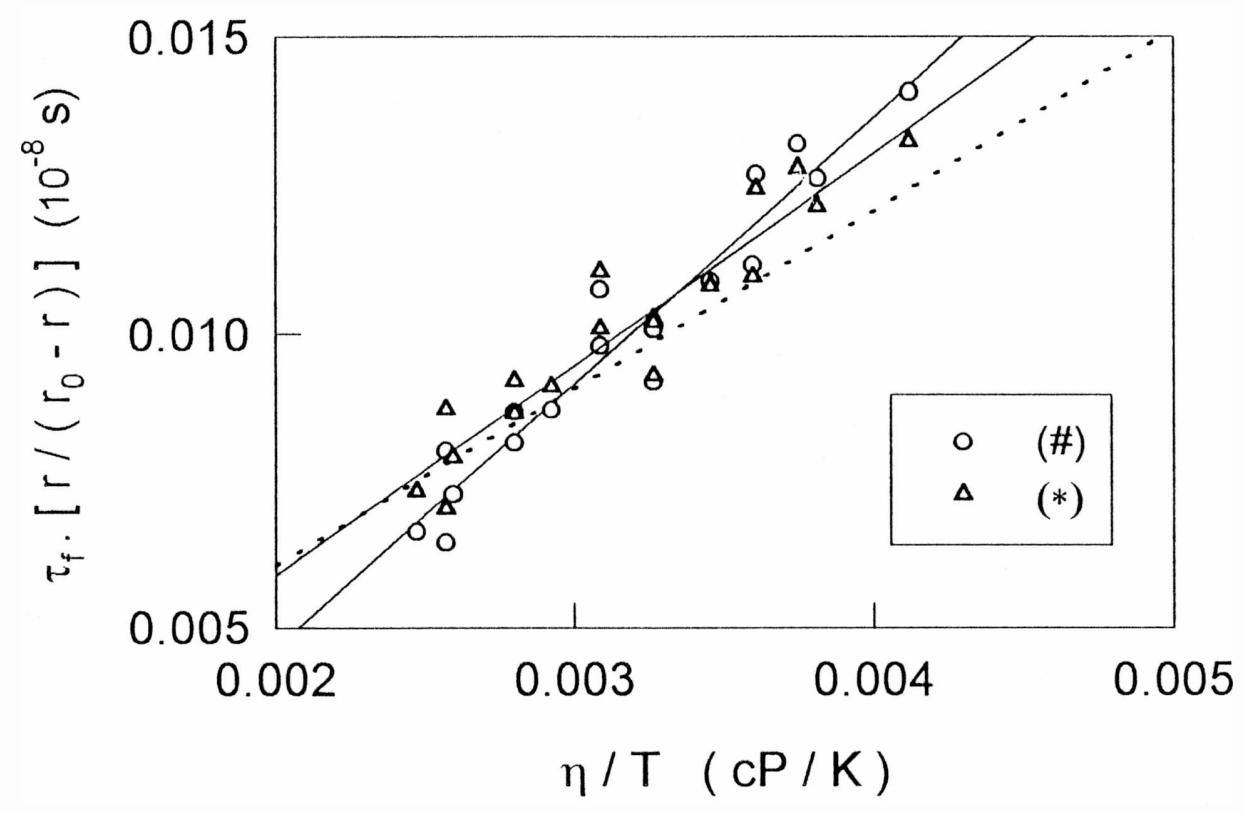

Figura 5.16

(*) $\quad \mathrm{k}_{\mathrm{r}}=3.1 \times 10^{8} 1 / \mathrm{s}, \quad \mathrm{A}_{\mathrm{nr}}=5.5 \times 10^{13} 1 / \mathrm{s}, \quad \mathrm{E}_{\mathrm{nr}}=5340 \mathrm{cal} / \mathrm{mol}$

(\#) $\quad\left(\mathrm{k}_{\mathrm{r}}=2.9 \times 10^{8} 1 / \mathrm{s}, \quad \mathrm{A}_{\mathrm{nr}}=5.2 \times 10^{14} 1 / \mathrm{s}, \quad \mathrm{E}_{\mathrm{nr}}=6680 \mathrm{cal} / \mathrm{mol}\right)$ 
El cambio ensayado está dentro del error experimental con que se determinan estos parámetros [Aramendía, 1994]. Si bien no se ha logrado ajustar completamente los puntos a la recta teórica, sí se ve que el cambio está en la dirección correcta, posibilitando un mejor acuerdo. No obstante debiera dejarse aclarado que no son precisamente las medidas de anisotropía un recurso apropiado para obtener a partir de éstas, parámetros tales como ser los parámetros de Arrhenius que se acaban de modificar, y que pueden ser determinados con mejor precisión siguiendo otros métodos. Sí es interesante, tal como se verá en lo que sigue con el análisis del DOCI, señalar la posibilidad que brinda la anisotropía de la fluorescencia, de poder estimar el tiempo de vida la molécula, cuando se asume como válida para ésta un determinado modelo hidrodinámico.

En el caso del DOCI, no se cuenta con una medida precisa de su tiempo de vida, pero hay medidas de su eficiencia de fluorescencia y de su tiempo de vida radiativo. Asumiendo como válido para DOCI el modelo usado con DTCI, el $\tau_{\mathrm{f}}$ que resulta se corresponde muy bien con aquellas medidas ( $\phi_{\mathrm{f}} \mathrm{y} \tau_{\mathrm{r}}$ ).

En la figura 5.17 se muestra la anisotropía medida de la fluorescencia del DOCI (círculos) en función del cociente temperatura/viscosidad; junto con el resultado (curvas en línea continua) de usar el modelo slip con un volumen molecular efectivo $25 \%$ mayor que el volumen de van der Waals de la molécula. Cada una de las curvas se corresponde con un tiempo de vida de la fluorescencia diferente. Así se tiene la curva (a) para $\tau_{\mathrm{f}}=228$ ps a $21^{\circ} \mathrm{C}$, (b) corresponde a $\tau_{\mathrm{f}}=114 \mathrm{ps}$ y $\left(\mathrm{c}\right.$ ) a $\tau_{\mathrm{f}}=57 \mathrm{ps}$, todos a la misma temperatura. De la figura podríamos decir que $\tau_{\mathrm{f}}=114 \mathrm{ps}$ es el valor correcto. Pero además es este valor el que resulta de hacer el producto $\phi_{\mathrm{r}}=0.052$ [Ponterini, 1992] $\times \tau_{\mathrm{r}}=2.2 \times 10^{-9} \mathrm{~s}$ [Apéndice C], obtenidos de la bibliografia. Se hace evidente de esta manera la similitud de comportamiento entre esta molécula y la de DTCI.

En la representación de los tiempos de difusión rotacional (figura 5.18), las rectas en línea a trazos corresponden a las regresiones lineales de los dos conjuntos de puntos que resultan de adoptar respectivamente, los tiempos de vida indicados para las curvas (a) y (c) en la figura anterior. La recta en línea continua, (b), corresponde al resultado que arroja el modelo propuesto para describir el comportamiento hidrodinámico de esta molécula. El mismo no depende de como se elige el tiempo de vida de la fluorescencia. Asumiendo entonces como válido el modelo que se usara para el DTCI, $\tau_{\mathrm{r}}=114 \mathrm{ps}$ a $21^{\circ} \mathrm{C}$ resulta ser claramente la mejor elección para lograr el buen acuerdo entre la teoría y las medidas experimentales. 


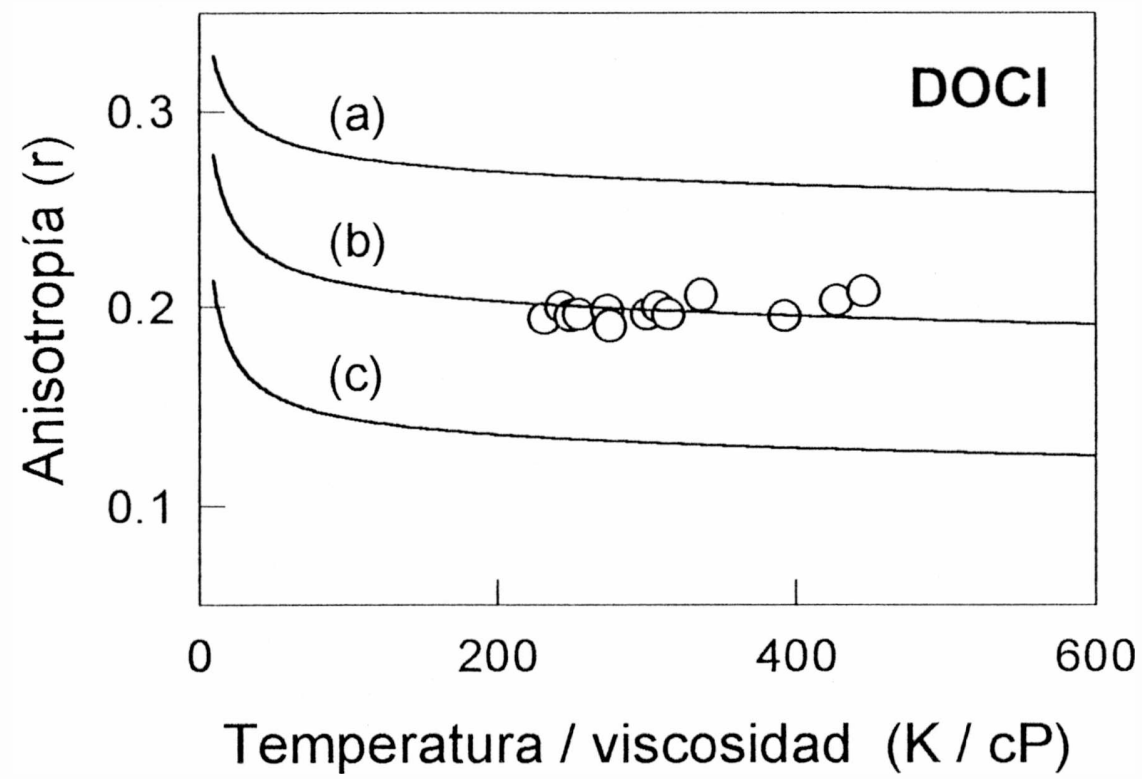

Figura 5.17. Curvas de anisotropia de la fluorescencia del DOCI correspondiendo a tiempos de vida diferentes: (a) $\tau_{f}=228 \mathrm{ps}$, (b) $\tau_{f}=114 \mathrm{ps}$, y (c) $\tau_{f}=57 \mathrm{ps}$.

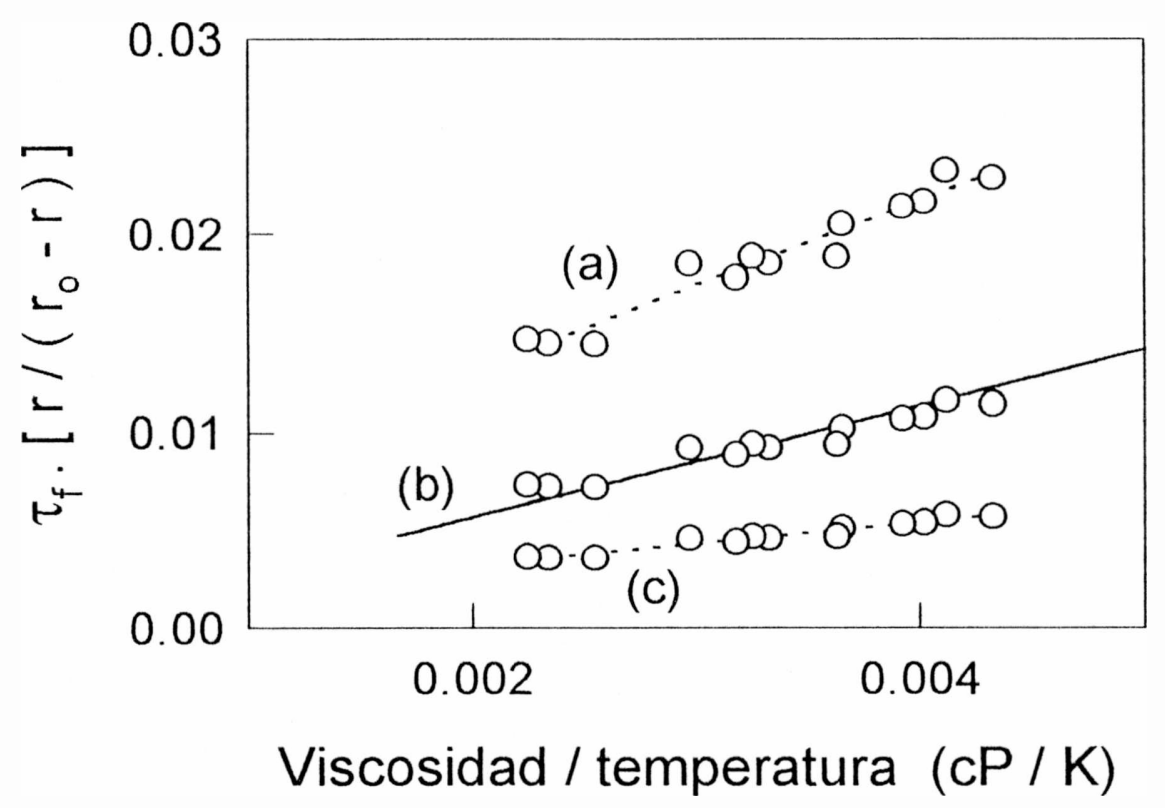

Figura 5.18 


\subsection{ANÁLISIS COMPARATIVO DE LA CUATRO CIANINAS ESTUDIADAS.}

Con el fin de facilitar el análisis en conjunto, acerca del comportamiento hidrodinámico de las especies normales de las cuatro cianinas estudiadas, se muestra en la figura 5.19 el resultado de trabajar bajos las mismas hipótesis referentes a los modelos hidrodinámicos escogidos, que permita inferir alguna conclusión sobre aquel comportamiento.

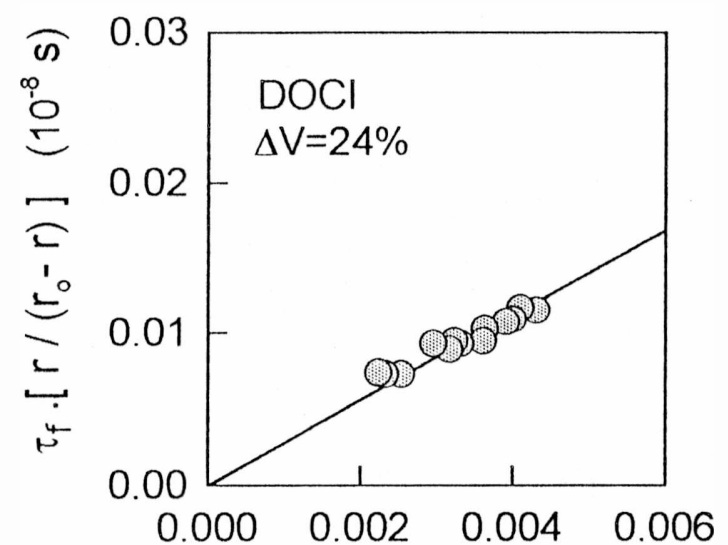

$\eta / \mathrm{T}(\mathrm{cP} / \mathrm{K})$

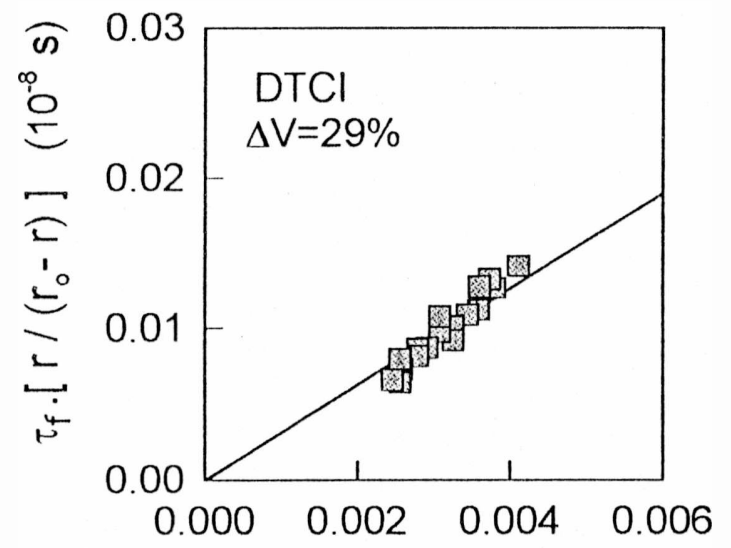

$\eta / \mathrm{T}(\mathrm{cP} / \mathrm{K})$

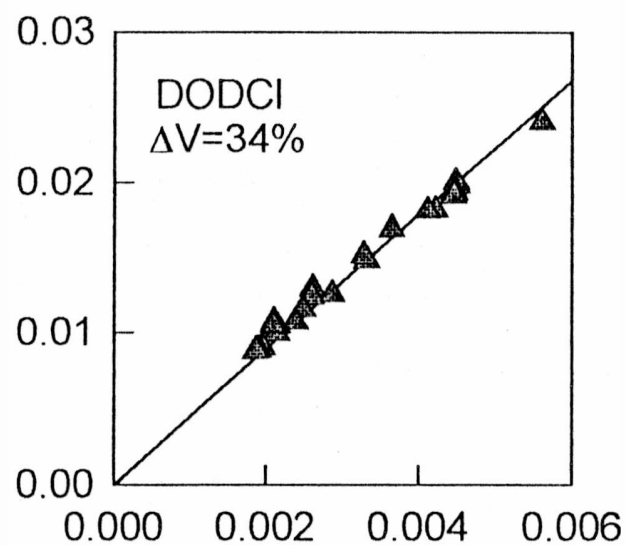

$\eta / \mathrm{T} \quad(\mathrm{cP} / \mathrm{K})$

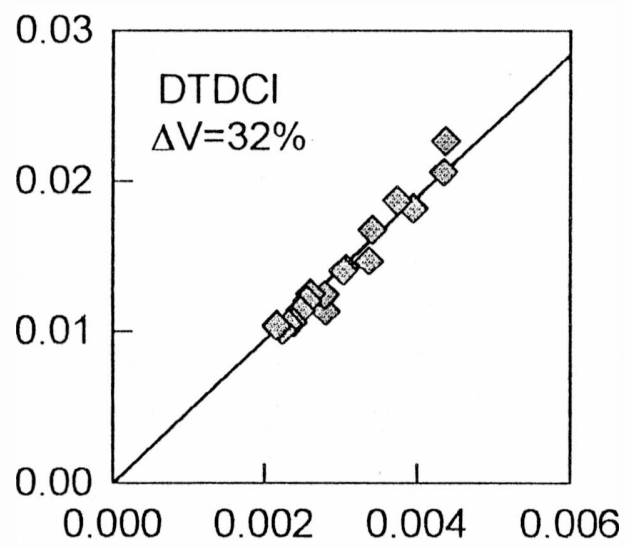

$\eta / \mathrm{T} \quad(\mathrm{cP} / \mathrm{K})$

Figure 5.19. Tiempos de difusión rotacional. $\Delta V$ representa el incremento de volumen que se requiere en cada caso para lograr el mejor ajuste. 
Los parámetros de Arrhenius que se usaron en la figura 5.19 para el tiempo de vida de la fluorescencia de estas especies ya fueron indicados oportunamente a lo largo de este capítulo, y se resumen en la tabla $C .1$ que aparece en el apéndice $C$. $\Delta V$ es el aumento de volumen, respecto del volumen de van der Waals de estas moléculas, que se requiere para alcanzar el mejor ajuste mostrado en la figura (líneas rectas)

En la tabla 5.3 se indica para cada molécula, su volumen de van der Waals y el volumen efectivo que resultó de ajustar los puntos calculados (tiempos de difusión rotacional)

Tabla 5.3

\begin{tabular}{|c|c|c|c|}
\hline Molécula & $V_{\text {eff }}\left(\AA^{3}\right)$ & $V_{\mathrm{o}}\left(\AA^{3}\right)$ & Razón \\
\hline DOCI & 372 & 301 & 1.24 \\
\hline DODCI & 449 & 335 & 1.34 \\
\hline DTCI & 419 & 324 & 1.29 \\
\hline DTDCI & 473 & 358 & 1.32 \\
\hline
\end{tabular}

Tabla 5.3. Volimenes moleculares de las cuatro cianinas estudiadas. $V_{\text {elr }}$ es el volumen molecular efectivo y $V_{\mathrm{o}}$ es el volumen de van der Waals.

Para DODCI resulta un incremento de volumen mayor que aquel que fuera estimado oportunamente (sección 5.2). Ello se debe a que el ajuste se limita a un intervalo de temperaturas más pequeño que en el primer caso. Esto se hizo así sólo a los efectos de presentar en un mismo rango de temperaturas $\left(0<\eta / \mathrm{T}<6 \times 10^{-3} \mathrm{cP} / \mathrm{K}\right)$ a las cuatro cianinas estudiadas. Como se puede apreciar en la tabla 5.3, los volúmenes efectivos que resultan para estas especies son cercanos en cada caso a sus volúmenes de van der Waals. En todos ellos, el incremento de volumen que se necesitó considerar para lograr el ajuste está en un mismo orden ( $29 \pm 5 \%$ ). A primera vista, esto nos estaría hablando de una interacción similar de las cuatro moléculas con el medio. La necesidad por considerar un volumen ligeramente mayor, será analizado en detalle en el próximo capítulo 
Parece evidente a partir de la figura 5.19, que la condición de contorno elegida para describir el comportamiento hidrodinámico de estas moléculas es la adecuada. Puede decirse que la difusión rotacional obedece la condición de contorno slip, con un volumen efectivo de rotación mayor que el volumen de van der Waals de estas moléculas. Si bien es cierto que la aplicación de la condición de contorno stick a la difusión rotacional de cualquier soluto, es equivalente a la aplicación de la condición slip con un volumen mayor, la diferencia de volumen ( volumen difusivo - volumen real de la molécula ) no es suficientemente grande como para que sea este el caso que se pueda considerar. Tal como ya fuera discutido para el DODCI, el uso de la condición de contorno stick en estas moléculas, requiere considerar un volumen difusivo menor que el volumen de van der Waals de las mismas, lo cual no puede ser real.

Que la condición de contorno slip sea la condición válida para estas cianinas, permite inferir la inexistencia de interacciones fuertes de las mismas con el medio. Luego se discutirá en detalle este punto. Volviendo a los gráficos de la figura 5.19, de la representación correspondiente al par DODCI-DTDCI puede concluirse que no existe un efecto importante, relativo a la difusión rotacional de estas moléculas, de los sustituyentes en los grupos terminales. Bajo las mismas condiciones, estas dos moléculas tienen un comportamiento similar, que trasladado al caso DOCI-DTCI, permitiría predecir (tal como se observa) el mismo comportamiento hidrodinámico entre estas dos cianinas.

El efecto del largo de la cadena polimetínica, contemplado en la elección del semieje mayor de los elipsoides con que se simulan estas moléculas, tampoco muestra un efecto que podamos distinguir. A partir de los resultados obtenidos se puede concluir que las cuatro moléculas estudiadas en solución, experimentan la misma fricción cuando rotan. 


\section{CAPÍTULO 6. INTERPRETACIÓN.}

En general, las moléculas de colorante con carga eléctrica, disueltas en solventes polares o con enlace hidrógeno, experimentan coeficientes de fricción grandes en comparación con solutos neutros de tamaño similar, disueltos en solventes de la misma viscosidad. A pesar de ello, todo sugiere hasta aquí que la hidrodinámica slip puede reproducir el comportamiento observado de las cianinas estudiadas en esta tesis. Si bien estas moléculas son monocationes, los resultados experimentales parecen ser consistentes con el modelo slip, de la misma manera que lo es el comportamiento de moléculas neutras con tamaños y formas similares a estas cianinas [Mateo, Fleming \#51].

La difusión rotacional es discutida en términos de la forma y el volumen molecular del soluto, la viscosidad del solvente; mientras que las interacciones intermoleculares específicas son excluidas del modelo hidrodinámico. Cuando estas interacciones están presentes, es de esperar que los tiempos de reorientación caigan entre las predicciones de los modelos slip y stick. Como ya fuera señalado, la hidrodinámica con condición de contorno slip puede predecir exitosamente los tiempos de reorientacion rotacional de muchas moléculas pequeñas en ausencia de interacciones fuertes soluto-solvente [Bauer]. Resultados obtenidos a partir de modelos teóricos sobre la dinámica de fluidos, sostienen que la hidrodinámica slip es más apropiada para representar el comportamiento de líquidos a escala molecular [Zwanzig]. Pero cuando la molécula de soluto es grande comparada con la del solvente, con una superficie rugosa a escala molecular o simplemente su forma es muy irregular, su comportamiento se espera se aproxime a aquel predicho por la hidrodinámica stick [Richardson]. Es evidente que un fuerte acoplamiento soluto-solvente podría conducir a la observación de un comportamiento stick como consecuencia del aumento del tamaño efectivo de la molécula de soluto. Entonces el grado de comportamiento slip-stick dependería simultáneamente de las características de la molécula de soluto y de la potencial interacción existente entre el soluto y el solvente.

Los coeficientes de difusión rotacional dependen de parámetros tales como volumen, temperatura, viscosidad, factores de forma. Cuando cambiamos la condición de contorno, todos estos parámetros no cambian. El efecto de pasar de la condición stick a slip es aumentar la difusión rotacional. Pero este mismo efecto podría lograrse, por ejemplo, considerando un volumen asociado a la molécula menor. O podría también ser resultado de una viscosidad menor que aquella que estamos tomando. La pregunta que surge es entonces a que factores atribuir el comportamiento hidrodinámico observado. Para poder responder a la misma, debemos tener en claro que representa verdaderamente cada parámetro y de que manera se relacionan entre sí.

Así por ejemplo, el volumen que aparece en la expresión del coeficiente de difusión rotacional no es simplemente el volumen de la molécula. Se trata del volumen del cuerpo en 
rotación. Esto es el de la molécula, sumado a todos aquellos elementos del fluido que puedan estar ligados directamente al soluto de modo de moverse solidariamente con él Podemos distinguir entonces un volumen intrínseco, que evaluamos mediante el uso de volúmenes de van der Waals, y un volumen de solvatación que estará asociado con la polaridad, electrostricción, interacciones dipolares, etc. En cualquier caso, es evidente que no podemos tomar como volumen del cuerpo, uno menor que el volumen de van der Waals correspondiente a la molécula. Un volumen mayor, dependerá de la interacción específica soluto-solvente. En solventes polares se podrán tener interacciones electrostáticas. Si el colorante es iónico existirán fuerzas iónicas atractivas con un solvente polar. Así, cualquier mecanismo que incremente la interacción entre el solvente y el soluto, jugará un rol importante en la difusión rotacional de la molécula de colorante.

En presencia de interacciones fuertes soluto-solvente, la predicción stick, más que la slip, debería estar en buen acuerdo con las observaciones. A pesar de estar trabajando con moléculas de colorante cargadas y un sistema soluto-solvente con puente hidrógeno, la predicción slip es consistente con el comportamiento observado. En general, las interacciones atractivas entre el soluto y el solvente deberían incrementar el rango sobre el cual sus movimientos están acoplados y en consecuencia conducir a un volumen efectivo del soluto mayor. Ajustar los tiempos de difusión rotacional medidos de moléculas cargadas con el modelo hidrodinámico slip, requiere en estos casos incrementar las dimensiones de los elipsoides usados en el modelado de aquellas moléculas. El mayor aumento en el volumen efectivo molecular está asociado con los colorantes dianiónicos, tal como fluoresceína. Pero cómo se explica este aumento en aquellos casos. Si bien se ha sugerido en algunas oportunidades que las interacciones por puente hidrógeno entre las moléculas de soluto y las de solvente son las causantes de tal comportamiento, el mecanismo responsable del aumento de los tiempos de difusión rotacional en estos sistemas, no parece que sea este. El éxito aparente de la condición de contorno slip para moléculas sin carga, sugirió el intento de explicar la difusión rotacional del dianión rosa de bengala como una desviación del comportamiento slip. Una posibilidad sería considerar la existencia de sitios especiales de interacción con el solvente [Spears], que afectan sólo una parte de la superficie molecular. La interacción con el solvente en estos sitios puede considerarse como una perturbación al coeficiente de fricción establecido con la condición de contorno slip. La hipótesis es que el solvente interactúa con los dos iones negativos del colorante, generando un torque que retarda su movimiento rotacional. Existe una fuerte interacción de los alcoholes con los sitios $\mathrm{O}^{-}$de rosa de bengala que apoya esta hipótesis y que podría explicar lo lento del decaimiento rotacional de los colorantes dianiónicos.

Los iones positivos son menos polarizables que los iones de oxígeno. Esto determina que una molécula cargada positivamente en un solvente alcohólico pueda tener un decaimiento orientacional más rápido, debido a un debilitamiento de la ligadura de las 
moléculas de solvente a aquellas de la estructura coordinada en torno al ion. De esta manera se podría en principio extender la explicación al caso de las cianinas estudiadas.

En la noción de sitios especiales de interacción con el solvente, cada tipo particular de sitio molecular generará un torque medio con el solvente. El efecto de la interacción ionalcohol dependerá de la coordinación media del alcohol. Si la coordinación alrededor de los iones es grande, aumentaría el tamaño medio de la molécula de soluto, reduciéndose entonces el efecto de torque sobre el solvente por el suavizado de las capas coordinadas. La capa de solvente coordinada ligada a un ion molecular no necesita considerarse como una capa rígida. Basta contar con una coordinación media que determine un nuevo volumen efectivo y un torque medio sobre el solvente. El tiempo de permanencia de una molécula de solvente en la estructura coordinada debe definirlo un modelo dinámico de intercambio. La energía necesaria para cambiar de un arreglo de solvatación a otro, puede ser muy pequeña aún cuando la energía de ligadura a la estructura de una sola molécula de solvente sea grande. El proceso de exclusión e inserción de nuevas moléculas en una estructura coordinada del solvente dependerá de la fuerza de ligadura a la primera capa. También el enlace de una molécula a un ion incrementa la fuerza de los enlaces hidrógeno con la segunda capa de moléculas de solvente. Se vuelve razonable entonces asumir que la energía de interacción soluto-solvente lo mismo que la energía de interacción media solventesolvente, serán parámetros importantes en un modelo cuantitativo que relacione estas energias de interacción dinámica con los torques medios que actúan contra la reorientación.

En contra de esta explicación cualitativa del fenómeno observado, esto es la necesidad por considerar volúmenes más grandes que los volúmenes de van der Waals de las cianinas estudiadas, debe señalarse la situación no real que supone en estas moléculas la localización de la carga catiónica en un sitio particular. La carga aparece distribuida en toda la molécula como muestran cálculos de estructura electrónica, que determinan las cargas parciales sobre cada átomo. Además en una situación así uno esperaría tener un comportamiento alineal con la temperatura, dada la dependencia con este parámetro de las estructuras de equilibrio en el seno del líquido, y tal como fue observado no es este el comportamiento que se advierte.

Cuando se analiza el comportamiento dinámico de un soluto en un dado solvente, el efecto del tamaño relativo del soluto puede ser un punto importante a considerar. Si la molécula de soluto es pequeña comparada con las moléculas del solvente podría ocurrir que esta viera facilitada su rotación. Uno esperaria que al ir aumentando el tamaño del soluto, aumente la fricción que éste experimente. En alcoholes podría pensarse que la red de enlaces hidrógeno forme cavidades o regiones donde la fricción se vea reducida. Esto podría explicar la difusión rotacional más rápida de solutos pequeños en alcoholes que en solventes 
simples (alkanos) y la aproximación de los tiempos de difiısión para solutos más grandes en estos solventes [Ben-Amotz].

El comportamiento sub-slip reportado en estudios de difusión rotacional cuando el volumen de la molécula de soluto llega a ser menor o comparable al de las moléculas de solvente, puede atribuirse a una falla en el uso de la hipótesis de un solvente continuo. Por ejemplo el soluto podría reorientarse muy rápidamente en los agujeros del solvente. El mismo comportamiento sub-slip de moléculas neutras pequeñas observado en n-alcoholes, puede ser interpretado como resultado de características dinámicas o estructurales de la solvatación microscópica del medio.

De acuerdo con la teoría de Eyring, un líquido es considerado como un arreglo más o menos regular de moléculas, en el cual ocasionalmente aparecen huecos. En un líquido normal, los huecos en la estructura de "red" se estiman en el 5\%. La existencia de los huecos en el solvente facilitaría el movimiento del soluto en este, reduciendo los coeficientes de fricción en mayor medida cuanto menor resultase el tamaño del soluto respecto del solvente. Los huecos no necesitan tener el tamaño y forma de la cavidad formada por el faltante de una molécula de la red. Estos pueden considerarse como espacios abiertos entre las moléculas, de tamaños que van desde la separación molecular de equilibrio a más de un diámetro molecular. Además pueden aparecer, desaparecer o moverse a través del sistema, todo como resultado del movimiento de la moléculas vecinas. La teoría de Eyring ha sido exitosa en predecir cualitativamente la difusión de moléculas pequeñas en estado líquido.

La teoría molecular de la microfricción de Gierer y Wirtz modifica la teoría de Stokes teniendo en cuenta la migración de las moléculas de soluto en los huecos del solvente. De esta manera aparece un factor debido a la microfricción que multiplica a la viscosidad en la ecuación de Einstein-Stokes, obteniéndose el correspondiente coeficiente para escala molecular. El factor de microfricción es menor que la unidad para solutos de tamaño menor o igual que las moléculas de solvente y se aproxima a uno para el caso de solutos grandes. Cuando se compara con los experimentos, el factor de microfricción llega en algunos casos a ser mayor que uno. Esto puede deberse a que las fuerzas entre las moléculas de solvente y el soluto son más fuertes que entre las mismas moléculas de solvente. Por el contrario, si las fuerzas entre el soluto y las moléculas de solvente fueran despreciables, se esperaría que la partícula pudiera cambiar de posición más fácilmente lo cual significa que el factor de microfricción se reduciría.

Si el acento se pone en la relación existente entre la fricción microscópica y la viscosidad de corte del solvente, partiendo de la condición slip se obtendrán comportamientos sub-slip y la explicación de la difusión rotacional de las moléculas estudiadas sólo puede abordarse desde este punto de vista, cambiando la condición de contorno hidrodinámica de slip a stick. Pero luego el factor de corrección que resulta para los coeficientes de fricción, dada la relación de tamaño soluto-solvente, es inferior a la que 
se requiere para poder explicar el comportamiento observado. La fricción microscópica no parece ser la causa determinante del comportamiento de nuestras cianinas, más aún cuando se compara el dispar comportamiento rotacional de estas cianinas con las moléculas de colorante dianiónicas antes señaladas. Es preferible entonces atender a otros fenómenos como posibles causas de la difusión rotacional observada.

Además de los cambios en el volumen efectivo de la molécula de soluto o la consideración de la viscosidad local del solvente, otros mecanismos tales como fricción dieléctrica pueden jugar un rol significativo en la determinación de los tiempos de difusión rotacional de moléculas de colorante. Esta fuente de fricción aparece como resultado de la disipación de energía por las moléculas de solvente cuando ellas se reorientan en respuesta a la difusión de la molécula de soluto. Se han hallado evidencias de la importancia de este mecanismo en la difusión rotacional de rodamina $6 \mathrm{G}$ y de otras moléculas de colorante cargadas, en solventes alcohólicos.

Antes de pasar a considerar la fricción dieléctrica, se discutirá el posible efecto del momento de inercia de las cianinas estudiadas sobre la difusión rotacional de las mismas

Cuando se incluye el momento de inercia de la molécula de soluto en el análisis de la despolarización de la fluorescencia, un término adicional aparece en la expresión del tiempo de difusión rotacional (Eq. 5.14). La nueva expresión tiene la siguiente forma [Kawski],

$$
\frac{\tau}{\frac{r_{0}}{r}-1}=\frac{V \eta}{k T} \cdot \frac{F}{S}+\frac{I}{6 k T \tau}
$$

I es el momento de inercia del soluto; los demás términos ya fueron definidos anteriormente Viendo esta expresión la pregunta que surge entonces es, ¿hasta dónde el acuerdo entre la teoría y el experimento dependerá del efecto inercial? Para responder a la misma basta simplemente con calcular el momento de inercia de las cianinas estudiadas y reemplazarlo en la ecuación (6.1), para ver cuál es el efecto que produce sobre las predicciones de los tiempos de difusión rotacional

Para ejemplificar bastará con considerar el caso de la especie normal del DODCI. Su momento de inercia calculado como el del elipsoide prolado que le fuera asignado (sección 5.2), con densidad constante, rotando alrededor de un semieje distinto del semieje mayor, es del orden: $I_{\mathrm{DODCl}} \cong 1.4 \times 10^{-43} \mathrm{Kg} \cdot \mathrm{m}^{2}$. Este valor del momento de inercia se corresponde muy bien con aquel que resulta a partir del tiempo de reorientación libre del rotor. En general, los tiempos de difusión rotacional salisfacen una expresión empírica sugerida por Bauer, 


$$
\tau_{\mathrm{rot}}=\mathbf{C} \cdot \boldsymbol{\eta}+\tau_{\mathbf{0}}
$$

donde $\mathrm{C}$ es una constante y $\eta$ es la viscosidad del solvente. La intersección para viscosidad cero, $\tau_{0}$, se asocia con el tiempo de reorientación libre del rotor, cuya expresión es como sigue:

$$
\tau_{0}=\frac{2 \pi}{9} \cdot \sqrt{\frac{\mathrm{I}}{\mathrm{kT}}}
$$

Para DODCl el valor medido de $\tau_{0}$ resultó ser 4 pseg [Waldeck \#85]. Luego, usando la última ecuación, el momento de inercia que resulta para la molécula es, $\mathrm{I}_{\mathrm{D} \text { onc: }} \cong 1.37 \times 10^{-43}$ $\mathrm{Kg} \cdot \mathrm{m}^{2}$, en acuerdo con el calculado anteriormente.

Ahora bien, el momento de inercia requerido para lograr el ajuste entre la teoría y el comportamiento hidrodinámico observado, puede ser obtenido comparando la ecuación (6.1) con aquella en la que no se considera el efecto inercial y el volumen considerado es el volumen efectivo que permite el ajuste (sección 5.5). Entonces, haciendo

$$
\begin{aligned}
& \frac{V_{0}}{k} \cdot \frac{\eta}{T} \cdot \frac{F}{S}+\frac{I}{6 k T \tau}=\frac{V_{\text {eff }}}{k} \cdot \frac{\eta}{T} \cdot \frac{F}{S} \\
& \therefore I=6 \frac{F}{S} \cdot\left(V_{\text {eff }}-V_{0}\right) \cdot \eta \cdot \tau
\end{aligned}
$$

Con condición slip y a temperatura ambiente, resulta para DODCI un momento de inercia del orden, $I_{D O D C I} \cong 1 \times 10^{-39} \mathrm{Kg} \cdot \mathrm{m}^{2}$. Este es cuatro ordenes de magnitud más grande que aquel estimado para esta molécula. Debería hablarse entonces de un momento de inercia efectivo, vinculado posiblemente con la fricción dieléctrica y la coordinación del solvente alrededor de la molécula de colorante. Pero si se adoptase un momento de inercia tal, dada la variación de el tiempo de vida de la fluorescencia de estas especies con la temperatura, se perdería la linealidad de la representación $\tau_{\text {rot }}$ vs $\eta / T$, que es el comportamiento real observado experimentalmente, no sólo en este caso sino en la inmensa mayoría. Esto conduce inmediatamente a dejar fuera de cualquier consideración el efecto inercial.

En solventes polares la fricción dieléctrica puede causar desviaciones del comportamiento hidrodinámico analizado hasta aquí. Resulta entonces de interés conocer el posible rol de la fricción dieléctrica en la difusión rotacional de las moléculas estudiadas.

En ausencia de efectos dieléctricos, la fricción mecánica gobierna la difusión rotacional de una molécula de soluto en solución. Introduciendo la fricción mecánica a través de ecuaciones hidrodinámicas continuas se obtuvo un modelo hidrodinámico en el cual el 
coeficiente de fricción es proporcional a la viscosidad de corte del solvente. Como ya hemos visto, el tiempo de difusión rotacional puede ser expresado por la siguiente ecuación,

$$
\tau_{\text {rot }}=\frac{\eta V}{k T} \cdot \frac{\mathbf{F}}{S}
$$

Luego, si la difusión rotacional está gobernada sólo por la hidrodinámica, $\tau_{\text {rot }}$ variará linealmente con el cociente viscosidad / temperatura, debiendo ser el factor de proporcionalidad independiente del solvente. En la búsqueda por hallar el efecto del solvente sobre la dinámica rotacional de la molécula de soluto, uno debe descifrar el rol de la fricción mecánica, de las interacciones dieléctricas e interacciones específicas soluto-solvente. Ha sido descartada la posibilidad de solvente "pegado" a la molécula de colorante y ahora sólo resta distinguir los efectos de la fricción dieléctrica sobre el comportamiento del sistema estudiado.

Hay varias formas en las cuales modelar la fricción dieléctrica. Esta puede tenerse en cuenta con un término adicional $\tau_{\mathbf{f a}}$, tal que

$$
\tau_{\mathrm{rot}}=\frac{\eta \mathbf{V}}{\mathrm{kT}} \cdot \frac{\mathbf{F}}{\mathrm{S}}+\tau_{\mathrm{fd}}
$$

Si bien esta aproximación aditiva no es del todo correcta, la misma brinda un marco simple para el análisis de la dinámica rotacional en solventes polares.

Para evaluar el término $\tau_{\mathrm{fd}}$ se puede usar el modelo dieléctrico continuo de Nee y $Z$ wanzig. Este considera un soluto dipolar, con un momento dipolar puntual $\boldsymbol{\mu}$, que rota lentamente en una cavidad esférica de radio $\mathbf{a}_{\mathbf{c}}$. El radio $\mathbf{a}_{\mathbf{c}}$ es usualmente estimado a partir del volumen molecular del soluto. La contribución de la fricción dieléctrica a la difusión rotacional está dada por

$$
\tau_{\mathrm{fd}}=\frac{\mu^{2}}{\mathbf{a}_{\mathrm{c}}^{3} \cdot \mathbf{k}} \cdot \frac{\varepsilon-1}{(2 \varepsilon+1)^{2}} \cdot \frac{\tau_{\mathrm{D}}}{\mathrm{T}}
$$

donde $\tau_{\mathrm{D}}$ es el tiempo de relajación de Debye y $\boldsymbol{\varepsilon}$ la constante dieléctrica del solvente. $\boldsymbol{\mu}$ es el momento dipolar de la cianina. El efecto de la fricción dieléctrica debería ser más notorio para el caso de pequeñas moléculas con momentos dipolares grandes

Una relación empírica para la fricción dieléctrica establece

$$
\frac{\varepsilon-1}{(2 \varepsilon+1)^{2}} \cdot \frac{\tau_{D}}{T}=A \cdot \frac{\eta}{T}+B
$$


Los parámetros A y B se obtienen de ajustar con una recta los puntos $\mathrm{f}(\varepsilon)$. $\tau_{\mathrm{D}} / \mathrm{T}$ vs $\eta / \mathrm{T}$. La justificación para esta relación, es la observación de que el tiempo de relajación de Debye se correlaciona linealmente con la viscosidad del solvente. En la figura 6.1 se muestra el ajuste realizado para etanol. La dependencia con la temperatura del tiempo de relajación dieléctico y de la constante dieléctrica del solvente fueron obtenidos de la bibliografia [Wiberg, Bessire]. Los parámetros que resultan de hacer la regresión lineal ( la recta en línea continua de la figura ) son: $\mathrm{A}=0.69 \mathrm{ps} / \mathrm{cP}$ y $\mathrm{B}=8.1 \times 10^{-4} \mathrm{ps} / \mathrm{K}$.

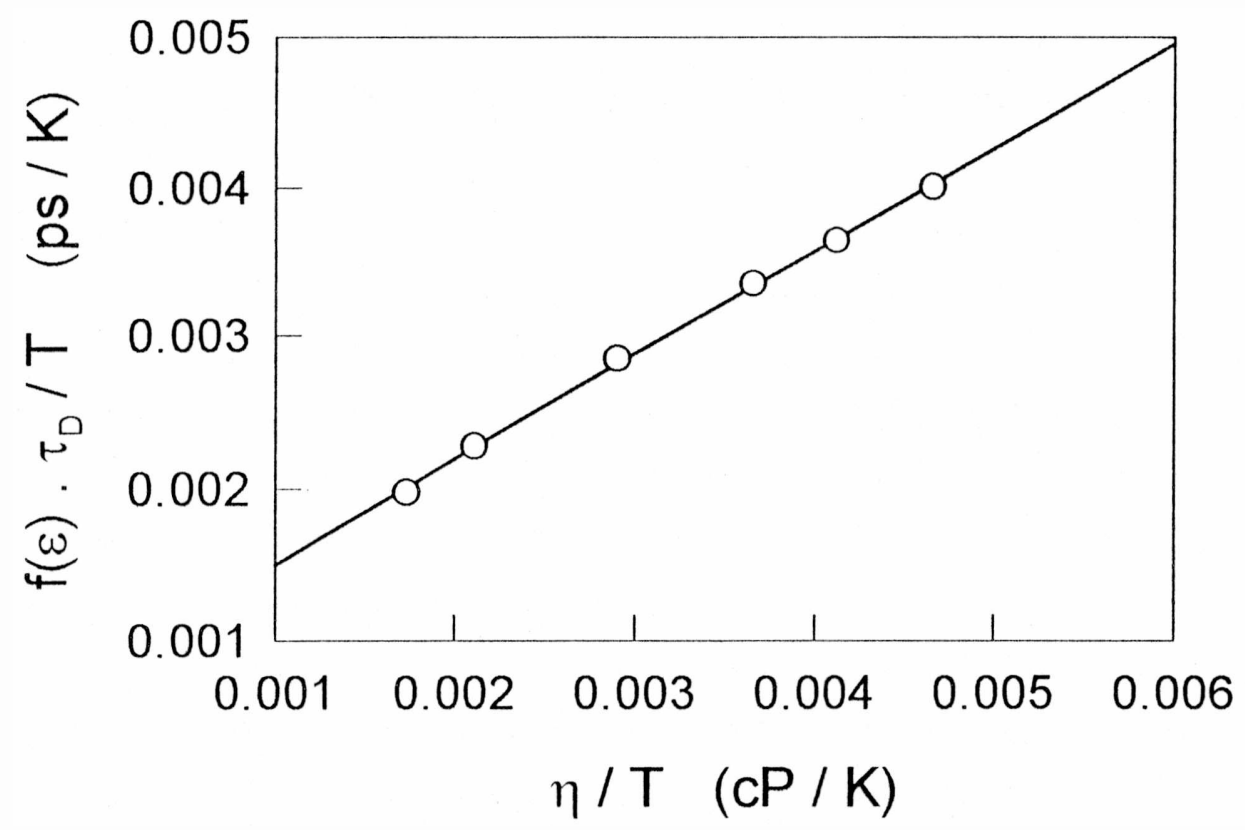

Figura 6.1

Combinando las ecuaciones $6.7,6.8$ y 6.9, resulta para el tiempo de difusión rotacional la siguiente expresión:

$$
\tau_{\text {rot }}=\left(\frac{\text { V.F }}{\text { k.S }}+\mathbf{A} \cdot \mathbf{P}\right) \cdot \frac{\eta}{T}+\text { B.P }
$$

$$
\text { donde } \quad \mathbf{P}=\frac{\mu^{2}}{\mathbf{a}_{\mathrm{c}}^{3} \cdot \mathbf{k}}
$$


Ahora en la representación de $\tau_{\text {rol }}$ vs $\eta / T$, la pendiente tiene contribuciones de la fricción mecánica y la dieléctrica. La desviación del comportamiento hidrodinámico debida a fricción dieléctrica, puede ser cuantificada en términos del parámetro A.P. Para ello se necesita conocer el momento dipolar.

Los momentos dipolares de la molécula, en el estado excitado y en el fundamental, pueden ser estimados a partir del corrimiento espectral inducido por el solvente en los máximos de absorción y de fluorescencia. Dentro de la descripción dieléctrica continua del solvente, el corrimiento Stokes está dado por la ecuación de Lippert, que relaciona este corrimiento con el cambio del momento dipolar,

$$
\text { hc } \cdot\left(v_{\mathrm{a}}-v_{\mathbf{f}}\right)=\left\{2\left[\mathbf{f}(\varepsilon)-\mathbf{f}\left(\mathbf{n}^{2}\right)\right] / \mathbf{a}_{\mathbf{c}}^{3}\right\} \cdot(\Delta \mu)^{2}+\text { cte }
$$

donde $v_{\text {a }}$ y $v_{\mathrm{t}}$ corresponden al máximo del espectro de absorción y fluorescencia, respectivamente. $\Delta \mu=\mu_{\mathrm{g}}-\mu_{\mathrm{e}}$ es la diferencia de los momentos dipolares. Las cantidades $\mathrm{f}(\varepsilon)$ y $f\left(n^{2}\right)$ son las funciones de Onsager, definidas por $f(x)=(x-1) /(2 x-1)$. $\varepsilon$ es la constante dieléctrica estática y $\mathbf{n}$ es el índice de refracción del solvente. La cantidad que aparece entre llaves es la polarizabilidad orientacional, asociada con la rotación de los momentos dipolares del solvente. Con esta ecuación, conociendo el momento dipolar del estado fundamental, es posible calcular el momento dipolar del estado excitado.

Un gráfico de $\Delta v\left(=v_{u}-v_{r}\right)$ como función de $\Delta f\left(=f(\varepsilon)-f\left(n^{2}\right)\right)$ debería ser lineal con pendiente proporcional a $(\Delta \mu)^{2}$. De acuerdo con los datos espectrales de las cianinas estudiadas y los parámetros del solvente, se advierte que las mismas deben tener momentos dipolares muy parecidos en sus estados fundamental y excitado. Esto es confirmado por cálculos de estructura electrónica, que determinan las cargas parciales localizadas sobre cada átomo de la molécula. El resultado para las cianinas estudiadas es de unos pocos Debye [Aramendía, com.]. En el caso particular del DODCI se establece para el momento dipolar de su especie todo-trans, un valor de 2.6 Debye en su estado excitado y 2 Debye para su estado fundamental. Otros isómeros de esta misma molécula no muestran cambios importantes respecto de estos valores. De afectar entonces la fricción dieléctrica a la difusión rotacional de estas moléculas, no debiera observarse cambio alguno cuando se estudia su movilidad orientacional ya sea en su estado excitado o en su estado fundamental. Aquí se ha seguido el comportamiento hidrodinámico de las cianinas a través de sus estados excitados, midiendo la despolarización de su fluorescencia. Pero un estudio de absorción anisotrópica del DODCI [Waldeck \#74, Shank] prueba que este comportamiento es independiente del estado observado. En aquel estudio se determinó un tiempo de difusión rotacional para la especie normal de esta cianina en su estado fundamental, que resulta indistinto del hallado 
para su estado excitado, confirmando así que la solvatación que pueda darse es muy similar en ambos casos.

Ahora bien, siguiendo con la especie normal del DODCI, en la figura 6.2 se muestran los tiempos de difusión rotacional obtenidos para un rango amplio de temperaturas (se corresponde con la figura 5.12), junto con las predicciones de los modelos hidrodinámicos

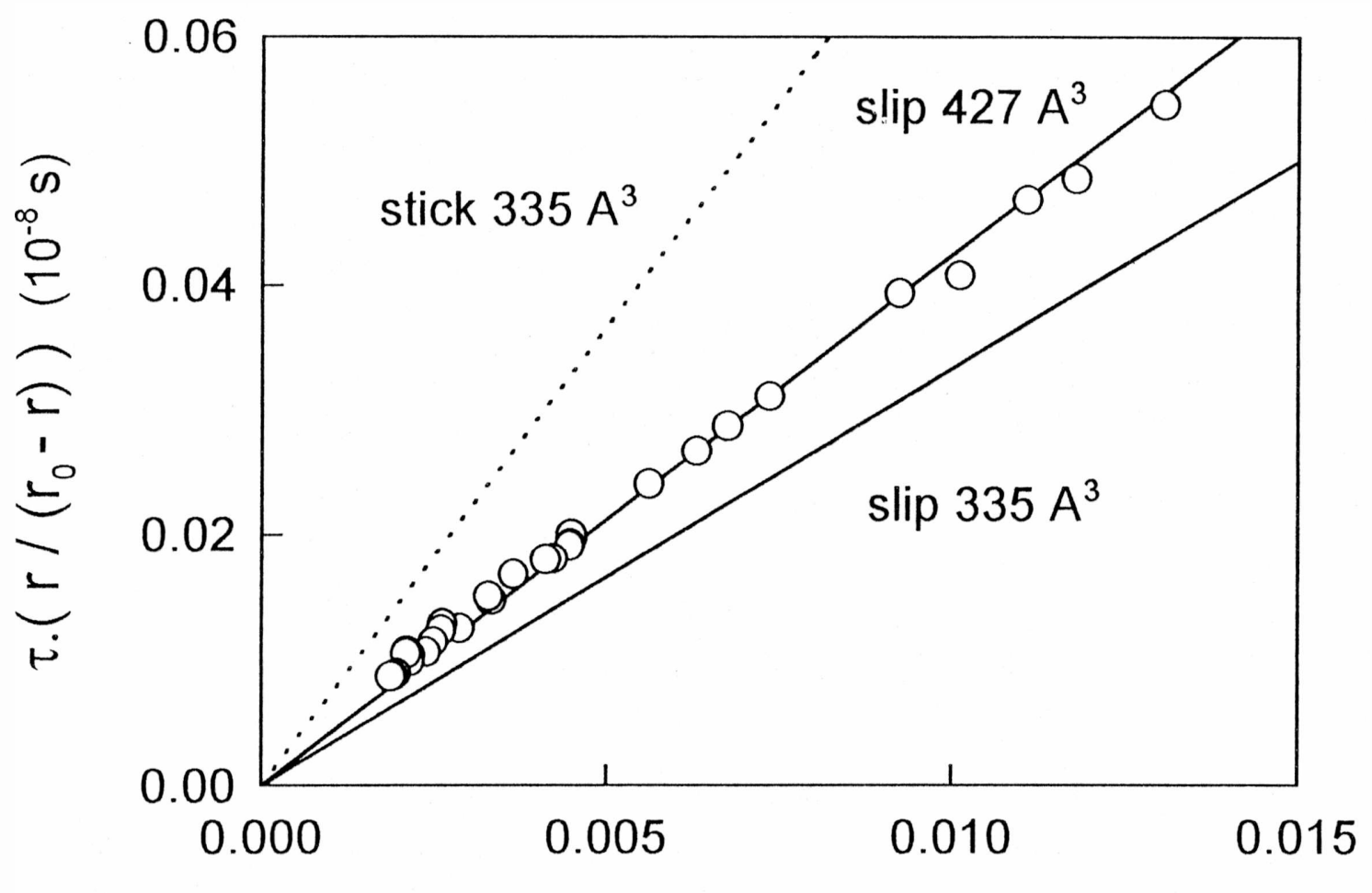

\section{$\eta / \mathrm{T} \quad(\mathrm{cP} / \mathrm{K})$}

Figura 6.2. Tiempos de difusión rotacional del DODCI junto con predicciones de los modelos hidrodinámicos slip (en linea continua) y stick (línea de puntos). El mejor ajuste (slip) se corresponde con un volumen efectivo de $427 A^{3}$.

slip-stick. El ajuste del tiempo de difusión rotacional como función del cociente $\eta / T$, requirió el uso de un volumen efectivo para esta molécula de aproximadamente un $27 \%$ más que el volumen de van der Waals de la misma. Si este incremento fuera atribuido a la fricción dieléctrica, de la ecuación (6.10) se tiene que,

$$
\text { P.A }=0.27 \times\left(V_{0} . \mathbf{F} / \mathbf{k . S}\right)
$$


Habiéndose determinado A para el solvente, P resulta:

$$
\mathrm{P}=\frac{0.27}{\mathrm{~A}} \cdot \tau_{\mathrm{rot}}^{\mathrm{slip}} \cdot \frac{\mathrm{T}}{\eta} \cong 1.5 \times 10^{4}\left[{ }^{\circ} \mathrm{K}\right]
$$

Estimando $\mathbf{a}_{\mathbf{c}}$ a partir del volumen de van der Waals de la molécula, se obtiene para su momento dipolar en el estado excitado un valor,

$$
\boldsymbol{\mu}=\left(\mathbf{P} \cdot \mathbf{a}_{\mathbf{c}}^{3} \cdot \mathbf{k}\right)^{\mathbf{1} / 2} \cong 13 \text { Debye }
$$

Este valor es grande comparado con el valor calculado (2.6 Debye), y uno inmediatamente pensaría en descartar el efecto dieléctrico sobre las moléculas estudiadas. Revisando el modelo de Nee y Zwanzig una de sus hipótesis consiste en suponer que el momento dipolar de la molécula es un dipolo puntual, ubicado en el centro de la misma. Así como se descartó la posibilidad de una carga puntual en un sitio particular de la molécula, tampoco podemos aceptar esta hipótesis como válida.

Para una distribución de carga arbitraria, en una cavidad esférica, la fricción dieléctrica tiene la misma forma indicada anteriormente, con el parámetro $\mathrm{P}$ estando dado por una expresión más compleja que depende de la distribución de carga real en la molécula de soluto. Alavi y Waldeck encontraron que cuando se utiliza una distribución de carga más cercana a la real, la fricción dieléctrica puede cambiar por varios ordenes de magnitud. En la tabla I de su trabajo se indican las diferencias cuantitativas entre coeficientes de fricción dieléctrica, calculados para dipolos puntuales y para una distribución de cargas extendida. De los resultados numéricos se desprende la importancia de la razón entre la distancia correspondiente a la separación de cargas y el radio de la cavidad dentro de la cual rota el dipolo. En la figura 6.3 puede apreciarse el cociente de los coeficientes de fricción dieléctrica en función de la razón $\mathbf{r} / \mathbf{a}_{\mathbf{c}}(\boldsymbol{\mu}=\mathbf{2 q r}$ ). Esta figura se contruyó a partir de los datos de la tabla I antes mencionada. Cuando el cociente $\mathbf{r} / \mathbf{a}_{\mathbf{c}}$ es 0.5 la fricción que experimenta el dipolo es más del doble de aquella que predice el modelo de Nee y Zwanzig. Para $\mathbf{r} / \mathbf{a}_{\mathbf{c}}>0.7$, llega a ser un orden más alta.

De esta manera se hace posible ajustar la desviación observada del comportamiento slip, considerando en el cálculo de la fricción dieléctrica una distribución de carga en la molécula de soluto más cercana a la situación real, que aquella correspondiente a considerar un dipolo puntual.

Resulta evidente de lo discutido hasta aqui, que la hidrodinámica slip con fricción dieléctrica es la mejor combinación para representar el comportamiento rotacional de las cianinas estudiadas en esta tesis. 


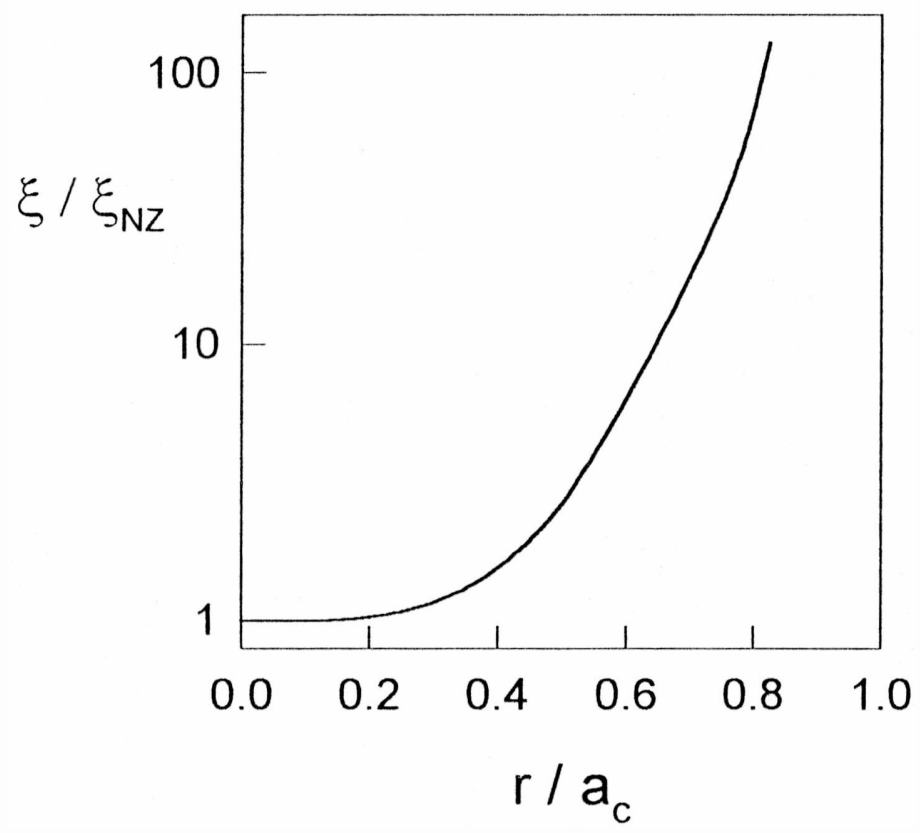

Figura 6.3. Cociente entre el coeficiente de fricción dieléctrica ( $\xi$ ) para un dipolo 2qr y aquel coeficiente corespondiente a un dipolo puntual de igual magnitud, colocado en el centro de la cavidad de radio $a_{c}$, como fiunción de $r / a_{c}$ 


\section{CAPÍTULO 7. CONCLUSIONES.}

- La hidrodinámica slip permite predecir el comportamiento rotacional de las cianinas estudiadas, cuando se adoptan elipsoides asimétricos para representar la estructura molecular de las mismas. Este modelo es consistente con la anisotropía de la fluorescencia medida de las distintas especies, a pesar de ser estas cianinas monocationes y no haberse observado en estos casos tal comportamiento con anterioridad al presente estudio

- En el caso particular de DODCI, este modelo ha permitido descartar la estructura di-cis para la especie normal de esta cianina, dando como muy probable la asignación todotrans para la misma. Una interacción ligeramente diferente entre las distintas especies de esta molécula y el solvente puede ser inferida de las medidas realizadas.

- El similar comportamiento hidrodinámico de las especies normales de las cianinas estudiadas, es consistente con la asignación de una estructura común a todas ellas (todo-trans), y demuestra que el largo de la cadena polimetínica o el heteroátomo en los grupos terminales, no tiene una influencia cualitativa en la difusión rotacional de estas moléculas.

- El estudio fotofisico realizado sobre las cianinas permitió establecer entre otros parámetros, la baja eficiencia de fluorescencia de sus fotoisómeros. Este hecho vuelve impracticable el estudio de polarización saturada, que resultara de cierta utilidad en el caso del DODCI.

- De los resultados obtenidos y su posterior interpretación, la fricción dieléctrica resulta ser el fenómeno más indicado para acompañar a la fricción mecánica en un modelo hidrodinámico que de cuenta de la difusión rotacional de las especies estudiadas.

- Para lograr una determinación precisa de la conformación de las distintas especies, la anisotropía de las mismas debería ser medida con técnicas de picosegundos, que posibiliten acceder a una medida directa de los tiempos de difusión rotacional. 
APÉNDICE A: EXPRESIÓN GENERAL PARA LA ANISOTROPIA DE LA FLUORESCENCIA.

En el capítulo 3 se señalaba que las intensidades de emisión observadas con polarización paralela y perpendicular a la excitación son:

$$
\begin{aligned}
& I_{p}(t)=\int_{\Omega} P_{c m}^{p} \cdot P(t) \cdot f(\Omega, t) d \Omega \\
& I_{s}(t)=\int_{\Omega} P_{e m}^{s} \cdot P(t) \cdot f(\Omega, t) d \Omega
\end{aligned}
$$

Si la emisión de las moléculas fluorescentes decae exponencialmente, con un tiempo de vida $\tau$, entonces $\mathrm{P}(\mathrm{t})=\exp (-\mathrm{t} / \tau)$ y puede ser puesto fuera de las integrales. Luego, la solución de las mismas es como sigue:

$$
\begin{aligned}
I_{p}(t)= & P(t) \cdot\left\{1 / 9+(4 / 15) \cdot g_{x} \cdot g_{y} \cdot h_{x} \cdot h_{y} \cdot \operatorname{Exp}\left[-\left(3 \cdot d_{z}+S\right) \cdot D \cdot t\right]+\right. \\
& (4 / 15) \cdot g_{y} \cdot g_{z} \cdot h_{y} \cdot h_{z} \cdot \operatorname{Exp}\left[-\left(3 \cdot d_{x}+S\right) \cdot D \cdot t\right]+ \\
& (4 / 15) \cdot g_{x} \cdot g_{z} \cdot h_{x} \cdot h_{z} \cdot \operatorname{Exp}\left[-\left(3 \cdot d_{y}+S\right) \cdot D \cdot t\right]+ \\
& (1 / 15) \cdot(\lambda+\omega) \cdot \operatorname{Exp}[-2 \cdot(S+U) \cdot D \cdot t]+ \\
& (1 / 15) \cdot(\lambda-\omega) \cdot \operatorname{Exp}[-2 \cdot(S-U) \cdot D \cdot t], \\
I_{s}(t)= & 1 / 6 P(t)-1 / 2 I_{p}(t)
\end{aligned}
$$

donde,

$$
\begin{aligned}
& \mathrm{S}=\mathrm{d}_{\mathrm{x}}+\mathrm{d}_{\mathrm{y}}+\mathrm{d}_{\mathrm{z}} \\
& U^{2}=d_{x}^{2}+d_{y}^{2}+d_{z}^{2}-d_{x} \cdot d_{y}-d_{x} \cdot d_{z}-d_{y} \cdot d_{z}, \\
& \lambda=\mathrm{g}_{\mathrm{x}}{ }^{2} \cdot \mathrm{h}_{\mathrm{x}}^{2}+\mathrm{g}_{\mathrm{y}}{ }^{2} \cdot \mathrm{h}_{\mathrm{y}}^{2}+\mathrm{g}_{\mathrm{z}}{ }^{2} \cdot \mathrm{h}_{\mathrm{z}}^{2}-1 / 3, \\
& \omega=\left(d_{x} / U\right) \cdot\left(g_{y}{ }^{2} \cdot h_{y}{ }^{2}+g_{z}{ }^{2} \cdot h_{z}^{2}-2 \cdot g_{x}{ }^{2} \cdot h_{x}{ }^{2}+h_{x}{ }^{2}+g_{x}{ }^{2}\right)+
\end{aligned}
$$




$$
\begin{aligned}
& \left(d_{y} / U\right) \cdot\left(g_{x}{ }^{2} \cdot h_{x}{ }^{2}+g_{z}{ }^{2} \cdot h_{z}{ }^{2}-2 \cdot g_{y}{ }^{2} \cdot h_{y}{ }^{2}+h_{y}{ }^{2}+g_{y}{ }^{2}\right)+ \\
& \left(d_{z} / U\right) \cdot\left(g_{x}{ }^{2} \cdot h_{x}{ }^{2}+g_{y}{ }^{2} \cdot h_{y}{ }^{2}-2 \cdot g_{z}{ }^{2} \cdot h_{z}{ }^{2}+h_{z}{ }^{2}+g_{z}{ }^{2}\right)-2 S / 3 U
\end{aligned}
$$

Entonces la expresión correspondiente a la anisotropía es:

$$
\begin{aligned}
r(t)= & \left(I_{p}-I_{s}\right) /\left(I_{p}+2 \cdot I_{s}\right) \\
r(t)= & (9 / 2) \cdot I_{p} / P(t)-1 / 2 \\
r(t)= & (6 / 5) \cdot g_{x} \cdot g_{y} \cdot h_{x} \cdot h_{y} \cdot \operatorname{Exp}\left[-\left(3 \cdot d_{z}+S\right) \cdot D \cdot t\right]+ \\
& (6 / 5) \cdot g_{y} \cdot g_{z} \cdot h_{y} \cdot h_{z} \cdot \operatorname{Exp}\left[-\left(3 \cdot d_{x}+S\right) \cdot D \cdot t\right]+ \\
& (6 / 5) \cdot g_{x} \cdot g_{z} \cdot h_{x} \cdot h_{z} \cdot \operatorname{Exp}\left[-\left(3 \cdot d_{y}+S\right) \cdot D \cdot t\right]+ \\
& (3 / 10) \cdot(\lambda+\omega) \cdot \operatorname{Exp}[-2 \cdot(S+U) \cdot D \cdot t]+ \\
& (3 / 10) \cdot(\lambda-\omega) \cdot \operatorname{Exp}[-2 \cdot(S-U) \cdot D \cdot t],
\end{aligned}
$$

Y en una forma más compacta,

$$
r(t)=\sum_{i=1}^{5} A_{i} \cdot \operatorname{Exp}\left[-a_{i} \cdot t\right]
$$

donde, como ya se mencionara, $\mathbf{a i}^{-1}$ son los tiempos de correlación rotacional. La anisotropía límite de la emisión está determinada por la suma de los factores preexponenciales $\mathbf{A}$.

En el caso particular de una molécula con dipolos de transición que yacen en un plano determinado por dos ejes de simetría molecular, la expresión correspondiente al decaimiento de la anisotropía se reduce a sólo tres decaimientos exponenciales. Estos son, los dos últimos en la expresión de arriba y uno de los restantes dependiendo de cuales sean los dos ejes de simetría. 


\section{APÉNDICE B : CONDICIÓN STICK-SLIP.}

Es en la evaluación de los coeficientes de fricción $(\zeta)$ donde aparece la condición de contorno soluto-solvente que uno adopta. Estos coeficientes se evalúan calculando el torque hidrodinámico que ejerce el fluido sobre el soluto en movimiento.

Si $\omega$ es la velocidad angular del elipsoide, el tensor de fricción viene definido por la siguiente relación de proporcionalidad:

$$
\mathbf{T}=-\zeta \cdot \omega
$$

El tensor de fricción es un tensor de segundo rango simétrico y es fácil ver que puede ser diagonalizado con una elección adecuada del sistema de coordenadas

Este mismo torque es determinado por la integral de superficie del momento del esfuerzo normal.

$$
\mathbf{T}=\oint \mathbf{d S} \cdot \mathbf{r} \times(\mathbf{n} \cdot \boldsymbol{\sigma})
$$

$\mathbf{r}$ es el vector posición de un elemento de superficie del elipsoide respecto del centro de este y $n$ un versor normal a aquel elemento de superficie. $\sigma$ es el tensor de esfuerzos.

Para un fluido incompresible, el tensor de esfuerzos tiene la forma simple:

$$
\sigma_{i j}=-p \cdot \delta_{i j}+\eta \cdot\left(\frac{\partial \mathbf{v}_{i}}{\partial \mathbf{x}_{j}}+\frac{\partial \mathbf{v}_{j}}{\partial \mathbf{x}_{i}}\right)
$$

donde $\mathbf{v}$ es la velocidad y $\mathbf{p}$ la presión del fluido. $\boldsymbol{\eta}$ es la viscosidad. En el caso más general, su expresión contiene un tercer término, en el que aparece un segundo coeficiente de viscosidad [Landau]

$\sigma_{i j}$ es la fuerza en la dirección i sobre un elemento de superficie perpendicular a j. $\sigma_{n}$ $(=n \cdot \sigma)$ es un vector, es la proyección de un tensor sobre $\mathbf{n}$, es la fuerza que actúa sobre la superficie normal a $\mathbf{n}$. En general tiene una componente en $\mathbf{n}$ y otra perpendicular. 
La condición de contorno hidrodinámica stick, es la condición convencional Establece que sobre la superficie del esferoide la velocidad del fluido es la misma que la de la superficie $(\mathbf{v}=\omega \times \mathbf{r})$.

La condición slip tiene dos partes. La primera es la condición de contorno para un fluido ideal. "El fluido no puede penetrar una superficie sólida". Entonces, sobre la superficie del elipsoide:

$$
\mathbf{v} \cdot \mathbf{n}=(\omega \times \mathbf{r}) \cdot \mathbf{n}
$$

Esta igualdad también se cumple con stick.

La segunda condición establece que no hay componente tangencial del esfuerzo normal sobre la superficie del elipsoide. La condición es entonces:

$$
\mathbf{n} \mathbf{x}(\mathbf{n} \cdot \sigma)=0
$$

Para obtener $\sigma$, se debe hallar la distribución de velocidades del fluido en la vecindad del cuerpo cuyo movimiento estamos estudiando. Para ello se resuelve la ecuación de Navier-Stokes alrededor del elipsoide, con la condición de contorno elegida sobre su superficie. Usando esta distribución de velocidades, se calcula el torque hidrodinámico total que actúa sobre el esferoide

La ecuación de Navier-Stokes es:

$$
\frac{\partial \mathbf{v}}{\partial \mathbf{t}}+(\mathbf{v} \cdot \nabla) \mathbf{v}=-\frac{1}{\rho} \nabla \mathbf{p}+\frac{\eta}{\rho} \Delta \mathbf{v}
$$

Para un movimiento estacionario y lento del fluido esta ecuación puede volver a escribirse como sigue:

$$
\eta \Delta \mathbf{v}=\nabla \mathbf{p}
$$

Esta ecuación es complementada con la condición $\nabla . \mathbf{v}=0$, que se sigue de la ecuación de continuidad cuando el fluido es incompresible.

La solución de este sistema de ecuaciones con condición slip, requiere el uso de un sistema de coordenadas curvilíneas. El sistema de ecuaciones puede reducirse entonces a uno más simple y ser resuelto con métodos numéricos 
En dinámica de fluidos las consideraciones fenomenológicas son macroscópicas. Las ecuaciones se aplican a elementos de fluidos que son suficientemente grandes como para que puedan definirse las variables termodinámicas del sistema, sin que llegue a ser necesario tener en cuenta interacciones de tipo intermolecular. El modelo que implica asumir como válidos los resultados que de aquí se desprenden, para el caso microscópico de la difusión rotacional, es tan sólo un modelo que busca no perder la "simplicidad" del tratamiento teórico desarrollado 
Asumiendo que el tiempo de vida radiativo de las especies normales de las cianinas estudiadas no depende de la temperatura, la dependencia con este parámetro del tiempo de vida de la fluorescencia es la misma que la de su eficiencia cuántica de fluorescencia:

$$
\tau(T)=\tau_{0} \cdot \phi_{f}(T)
$$

La inversa del tiempo de vida del estado excitado es la suma de las velocidades de desactivación de aquel estado,

$$
1 / \tau=\mathrm{k}_{\mathrm{r}}+\mathrm{k}_{\mathrm{nr}}
$$

La constante del decaimiento radiativo $\left(\mathrm{k}_{\mathrm{r}}\right)$ es $\tau_{0}{ }^{-1}$, y para el decaimiento no radiativo se asume un proceso activado, de la forma:

$$
\mathrm{k}_{\mathrm{nr}}=\mathrm{A}_{\mathrm{nr}} \cdot \mathrm{e}^{-\frac{\mathrm{E}_{\mathrm{m}}}{\mathrm{RT}}}
$$

donde la energía de activación $\left(E_{n r}\right)$ y el factor preexponencial se suponen independientes de la temperatura.

$$
\begin{aligned}
& \text { De (C. 1) y (C. 2) se tiene, } \\
& \mathrm{k}_{\mathrm{nr}}=\mathrm{k}_{\mathrm{r}} \cdot\left(\frac{1}{\phi_{\mathrm{r}}}-1\right)
\end{aligned}
$$

Luego,

$$
\ln \left(\frac{1}{\phi_{\mathrm{f}}}-1\right)=\ln \left(\frac{\mathrm{A}_{\mathrm{nr}}}{\mathrm{k}_{\mathrm{r}}}\right)-\frac{\mathrm{E}_{\mathrm{nr}}}{\mathrm{RT}}
$$

Graficando el primer miembro de la ecuación anterior (C.5) en función de la inversa de la temperatura, se debería obtener una recta de la cual se pueda extraer los parámetros de Arrhenius, $A_{u r}$ y $E_{u r}$, conociendo $k_{r}$. Si bien todos estos parámetros pueden ser encontrados en la bibliografia, algunos han sido determinados aquí midiendo la variación del máximo de fluorescencia de estas especies con la temperatura, en el intervalo correspondiente a las medidas de polarización. 
La señal fluorescente a una dada temperatura, I(T), puede ser considerada proporcional a la eficiencia de fluorescencia del compuesto a esa temperatura. Luego, con una valor conocido de $\phi_{\mathrm{f}}$ se puede determinar $\phi_{\mathrm{l}}(\mathrm{T})$ mediante la siguiente relación:

$$
\phi_{\mathrm{f}}(\mathrm{T})=\frac{\mathrm{I}(\mathrm{T})}{\mathrm{I}\left(\mathrm{T}_{0}\right)} \cdot \phi_{\mathrm{f}}\left(\mathrm{T}_{0}\right)
$$

En la figura C.1 se muestra el resultado obtenido de representar gráficamente la ecuación (C.5), con $\phi_{\mathrm{f}}(\mathrm{T})$ dada por (C.6), para DTDCI disuelto en etanol. En la determinación de la variación de la eficiencia de fluorescencia con la temperatura, se utilizó el mismo arreglo experimental con el que se midieron las componentes polarizadas de las intensidades de fluorescencia. En lugar del sistema de colimación, se usó un monocromador posicionado en el máximo de fluorescencia. El polarizador usado antes como analizador se colocó en ángulo mágico para evitar el efecto de la despolarización sobre las medidas de eficiencia de fluorescencia.

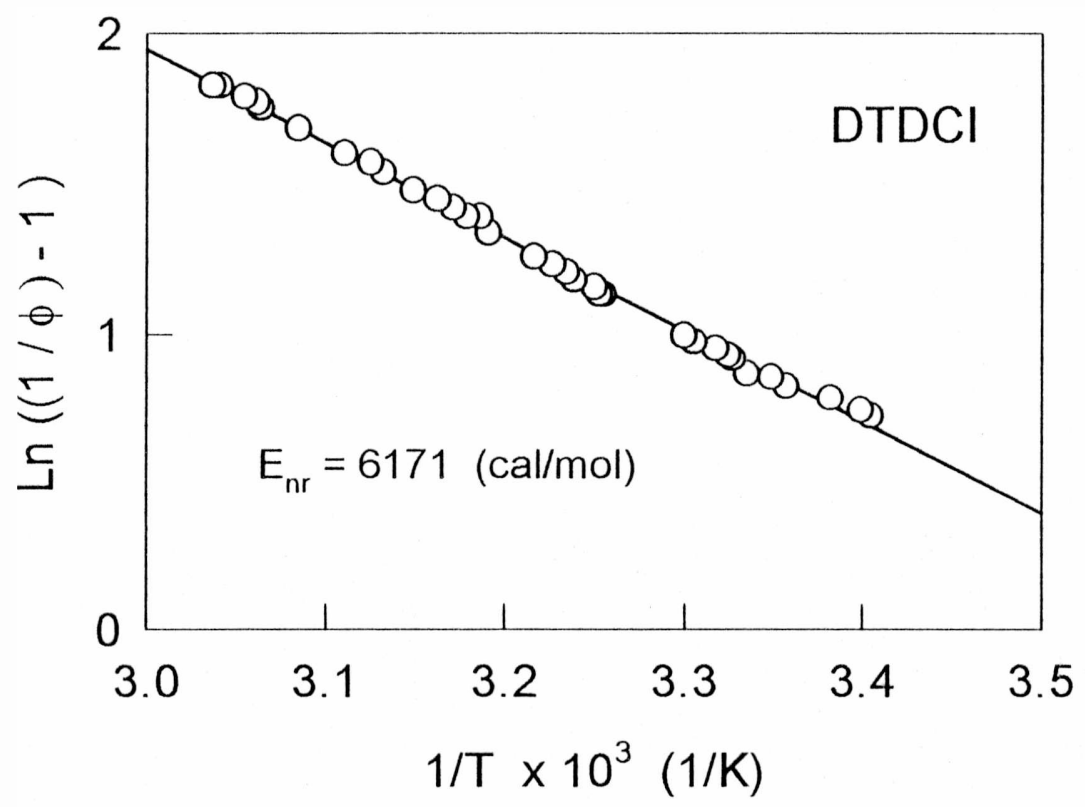

Figura C. 1

Para que la igualdad (C.6) sea válida no deben producirse cambios con la temperatura en la distribución espectral de la emisión o cambios en otros factores que 
vuelvan dependiente con la temperatura la proporcionalidad entre la eficiencia de nuorescencia y la intensidad de esta. Así por ejemplo, la variación de la absorbancia de las muestras con la temperatura puede influir en la determinación de $\phi_{\mathrm{l}}(\mathrm{T})$. Cuando la temperatura aumenta el solvente se expande, la concentración baja y la absorbancia disminuye. Para corregir la influencia de este factor simplemente se multiplica el segundo miembro de la ecuación (C.6) por $\mathrm{A}\left(\mathrm{T}_{0}\right) / \mathrm{A}(\mathrm{T})$, siempre que las absorbancias sean pequeñas $(A<0.05)$. En el intervalo de temperaturas considerado, la corrección es típicamente del $5 \%$ y no tiene mayor efecto en el cálculo de las energías de activación [Negri]

A continuación se detallan los parámetros utilizados en el capítulo 5, que establecen la dependencia con la temperatura del tiempo de vida de fluorescencia de las distintas especies estudiadas.

Tabla C. 1

\begin{tabular}{|c||c|c|c|c||}
\hline Molécula & $\mathrm{K}_{\mathrm{r}} \times 10^{8}(1 / \mathrm{s})$ & $\mathrm{A}_{\mathrm{rr}} \times 10^{13}(1 / \mathrm{s})$ & $\mathrm{E}_{\mathrm{nr}}(\mathrm{kcal} / \mathrm{mol})$ & $\tau_{\mathrm{r}}{ }^{*}(\mathrm{~ns})$ \\
\hline DODCI & 3,94 & 0,63 & 5,48 & 1,09 \\
\hline DOCI & 4,6 & 2,5 & 4,7 & 0,12 \\
\hline DTDCI & 2,6 & 2 & 6,17 & 1,3 \\
\hline DTCI & 2,9 & 52 & 6,68 & 0,173 \\
\hline
\end{tabular}

* $\tau_{\mathrm{f}}$ es el tiempo de vida de la fluorescencia, a $20^{\circ} \mathrm{C}$, que resulta de usar la ecuación (C.2) con los parámetros dados en esta tabla.

Con los valores de $\mathrm{K}_{\mathrm{r}}$ obtenidos de la bibliografia y las energias de activación medidas, se determinaron los parámetros de Arrhenius $A_{n r}$ tal que el tiempo de vida resultante coincida con aquel que se indica en la tabla C.1 y que están en acuerdo con datos de la bibliografia como puede ser visto en la tabla siguiente (C.2). En el caso del DODCI, todos los parámetros adoptados corresponden a los publicados en el trabajo de Velsko

En la tabla C.2 se indican distintos parámetros de fluorescencia extraídos de la bibliografia, que tienen que ver con la elección de aquellos parámetros que aparecen en la tabla anterior. Los tiempos de vida radiativo fueron calculados usando la fórmula de Strickler y Berg, u otra similar a aquella expresión. Los tiempos de vida de fluorescencia que aparecen en esta tabla han sido medidos con técnicas de picosegundos. En todos los casos el solvente es etanol. 
Tabla C.2

\begin{tabular}{|c|c|c|c|c|c|}
\hline Molécula & $\tau_{\mathrm{o}}(\mathrm{ns})$ & $\phi_{\mathrm{r}}$ & $\tau(\mathrm{ns})$ & Temp. $\left({ }^{\circ} \mathrm{C}\right)$ & Referencia \\
\hline \multirow{5}{*}{ DTCI } & 3.7 & 0.071 & & 25 & Aramendia \\
\hline & 3.23 & & & $\mathrm{~T}_{\text {amb. }}$ & Vaveliuk $^{(\cos )}$ \\
\hline & & & 0.241 & 25 & Sibbett \\
\hline & & & 0.173 & 20 & Winkworth \\
\hline & & & 0.140 & $22 \pm 3$ & Gadonas \\
\hline \multirow{3}{*}{ DOCI } & 2.17 & 0.12 & & 25 & Aramendía \\
\hline & 2.24 & & & $\mathrm{~T}_{\mathrm{amb}}$ & Vaveliuk $^{(\mathrm{com})}$ \\
\hline & & 0.052 & & 21 & Ponterini ${ }^{(1992)}$ \\
\hline \multirow{4}{*}{ DTDCI } & 4.17 & 0.35 & & 25 & Aramendía \\
\hline & & & 1.278 & 25 & Sibbett \\
\hline & 4.2 & & 1.5 & & Sundström \\
\hline & 3.43 & 0.35 & & & Dempster \\
\hline \multirow{7}{*}{ DODCI } & 2.54 & 0.49 & & & Dempster \\
\hline & & & 1 & & Adams \\
\hline & & & 1.2 & 20 & Jaraudias \\
\hline & & $0.42^{*}-0.48 \dagger$ & 1.15 & & Madge \\
\hline & & & 1.2 & & Mialocq $^{(1976)}$ \\
\hline & & & 1 & $\mathrm{~T}_{\text {amb. }}$ & Heisel \\
\hline & 2.54 & & 1.09 & 20 & Velsko \\
\hline
\end{tabular}

$†$ Medida respecto a Rodamina $6 \mathrm{G}$

* Medida respecto a fluoresceina sódica.

En el caso del DODCI no se observan diferencias importantes entre los distintos valores reportados por los diferentes autores. Para DTECI la situación es similar, si bien en este caso las fuentes de información de que se dispone son menos numerosas comparadas con el primer colorante.

Para DOCI y DTCI los dos valores calculados del tiempo de vida radiativo son, en cada caso, muy parecidos. En el caso del DOCI sí se observa que la diferencia de eficiencias de fluorescencia medida por distintos autores es importante. Si bien la medida 0.12 es en etanol $95 \%$, que debería ser algo mayor que aquella en etanol puro, su diferencia con el valor 0.052 no puede ser explicada. Atendiendo a la similitud de tiempos de vida de 
fluorescencia entre DODCI y DTDCI, uno esperaría que DOCI y DTCI tuvieran tiempos de vida similares. La eficiencia de fluorescencia 0.052 parece estar en mejor acuerdo con el cálculo del tiempo que resulta de multiplicar esta eficiencia por el tiempo de vida radiativo.

La dependencia de la viscosidad del solvente (etanol absoluto) con la temperatura fue obtenida a partir de datos de la bibliografia [Weast]. La expresión usada para establecer dicha relación es de la forma:

$$
\eta=\eta_{0} \cdot \exp \left(E_{\eta} / R T\right)
$$

Los parámetros de Arrhenius escogidos fueron:

$$
\begin{aligned}
& \boldsymbol{\eta}_{\mathbf{0}}=2,610^{-3} \mathrm{cP} \\
& \mathbf{E}_{\boldsymbol{\eta}}=3580 \mathrm{cal} / \mathrm{mol}
\end{aligned}
$$


DODCI

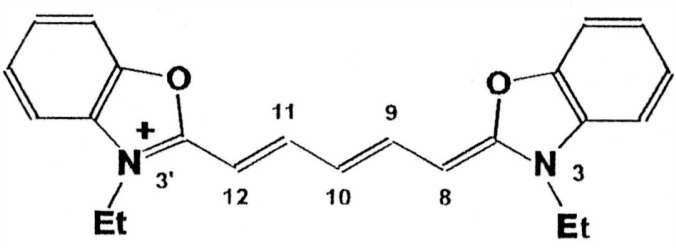

TODO-TRANS

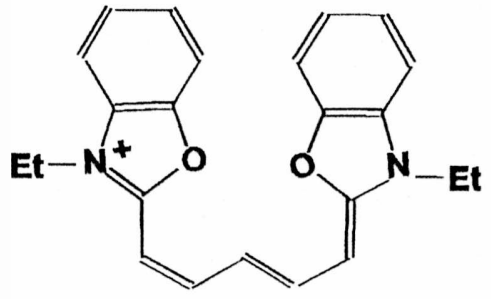

8,9-11,12 DI-CIS

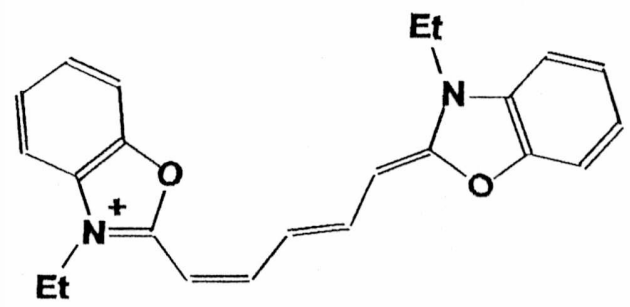

11,12 MONO-CIS

En la figura 2.3 (pag. 23) se representa al isómero todo-trans de las cianinas estudiadas. Para el caso del DODCI (ioduro de 3,3'-dietiloxadicarbocianina) se indican a continuación los tres isómeros de este colorante, que fueron motivo de análisis en el capítulo 5. La estructura mono-cis que se muestra, resulta de la rotación en $\pi$ de una parte de la molécula alrededor del enlace 11,12 de la cadena polimetínica. Para lograr la estructura di-cis se requiere hacer una segunda rotación, ahora en torno al enlace 8,9 de la misma cadena. 


\section{REFERENCIAS.}

Adams, M.C., Bradley, D.J., Sibbett, W., Springer Series in Chem. Phys. 4,

Picosecond Phenomena, Ed. F.P. Schäfer, Springer-Verlag Berlin, 1978, pag. 108

Alavi, D.S., Waldeck, D.H., J. Chem. Phys., 94, 1991, 6196.

Albrecht, A.C., J. Mol. Spectry, 6, 1961, 84.

Anfinrud, P.A., Struve, W.S., J. Chem. Phys., 87, 1987, 4256.

Aramendia, P.F., Negri, M., San Roman, E., J. Phys. Chem., 98, 1994, 3165

Aramendía, P.F., comunicación privada.

Arthurs, E.G., Bradley, D.J., Roddie, A.G., Appl. Phys. Lett., 20, 1972, 125.

Arthurs, E.G., Bradley, D.J., Roddie, A.G., Chem. Phys. Lett., 22, 1973, 230.

Awad, M.M., McCarthy, P.K., Blanchard, G.J., J. Phys. Chem., 98, 1994, 1454.

Bauer, D.R., Brauman, J.I., Pecora, R., J. Am. Chem. Soc., 96, 1974, 6840

Bäumler, W., Penzkofer, A., Chem. Phys., 142, 1990, 431.

Bäumler, W., Penzkofer, A., Chem. Phys., 140, 1990, 75.

Ben-Amotz, D., Scott, T.W., J. Chem. Phys., 87, 1987, 3739.

Bessire, D.R., Quitevis, E.L., J. Phys. Chem., 98, 1994, 13083.

Bilmes, G.M., Tocho, J.O., Braslavsky, S.E., J. Phys. Chem., 92, 1988, 5958.

Bilmes, G.M., Tocho, J.O., Braslavsky, S.E., J. Phys. Chem., 93, 1989, 6696.

Bolton, J.R., Archer, M.D., Am. J. of Phys., 1991

Bondi, A., J. Phys. Chem., 68, 1964, 441

Chen, R.F., Bowman, R.L., Science, 147, 1965, 729

Chuang, T.J., Eisenthal, K.B., J. Chem. Phys., 57, 1972, 5094.

Churio, M.S., Angermund, K.P., Braslavsky, S.E., J. Phys. Chem., 98, 1994, 1776.

Churio, M.S., Angermund, K.P., Braslavsky, S.E., J. de Phys. IV C7, 4, 1994, 323.

Dempster, D.N., Morrow, T., Rankin, R., Thompson, G.F., J. Chem. Soc. Faraday Trans. II $68,1972,1479$.

Duchowicz, R., Scaffardi, L., Tocho, J.O., Chem. Phys. Lett., 170, 1990, 497. 
Dietz, F., Rentsch, S.K., Chem. Phys, 96, 1985, 145.

Drexhage, K.H., Structure and properties of laser dyes. Dye Lasers, 2nd Edition, Editor F.P.Schäfer; Springer-Verlag, Berlin Heidelbery New York, 1977

Edward, J.T., Journal of Chemical Education, 47, 1970, 261

Edwardes, D., Quart. J. Pure Appl. Math., 26, 1892, 70.

Eisenthal, K.B., Drexhage, K.H., J. Chem. Phys,, 51, 1969, 5720.

Favro, L., Phys. Rev., 119, 1960, 53

Fleming, G.R., Knight, A.E.W., Morris, J.M., Robbins, R.J., Robinson, G.W., Chem. Phys. Letters, 49, 1977, 1.

Fleming, G.R., Knight, A.E.W., Morris, J.M., Robbins, R.J., Robinson, G.W., Chem. Phys. Letters, 51, 1977, 399.

Fork, R.L., Shank, C.V., Yen, R., Hirlimann, C., IEEE J. Quant. Electron., QE19, 1983, 500 .

Gadonas, R., Danelyus, R, Piskarskas, A., Rentsch, S., Sov. J. Quantum Electron., $13(2), 1983,186$

G.H.C. New Reports on Progress in Physics, 46(8), 877, 1983

Gierer, A., Wirtz, K., Z. Naturforsch., 8a, 1953, 532.

Grieser, F., Lay, M., Thistlethwaite, P.J., J. Phys. Chem., 89, 1985, 2065

Heisel, F, Miene, J.A., Sipp, B., J. Luminescence, 24, 1981, 651.

Henrichs, P.M., Gross, S., J. Am. Chem. Soc., 98, 1976, 7169.

Hu, C., Zwanzig, R J., J. Chem. Phys., 60, 1974, 4354

Jaraudias, J., J. Photochem., 13, 1980, 35

Kaliteevskaya, E. N., Razumova, T.K., Opt Spectrosc. (Engl. Transl.), 43, 1977, 398

Kawski, A., Critical Rev. Analytical. Chem., 23, 1993, 459.

Knudtson, J., Eyring, E., J. Phys. Chem., 78, 1974, 2355.

Kolesnikov, A.M., Mikhailenko, F.A., Russ. Chem. Rev., 56 (3), 275, 1987.

Landau, L.D., Fifshitz, E.M. Mecánica de Fluidos, vol 6, Curso de Física Teórica. Editorial Reverté, 1978 
Lessing, H.E., Optical and Quantum Electronics, 8, 1976, 309.

Lessing, H.E. and Von Jena, A., B6, pag.753, Continuous Picosecond Spectroscopy of Dyes. Laser Handbook, edited by M.L. Stitch, North-Holland Publishing Company, 1979.

Levitus, M., Negri, R.M., Aramendía, P.F., J. Phys. Chem., 99, 1995, 14231.

Lippert, E., Z. Elektrochem., 61, 962, 1957

Lombardi, J.R, Raymonda, J.W. and Albrecht, A.C., J. Chem. Phys., 40, 1964, 1148.

Madge, D., Windsor, M., Chem. Phys. Lett., 27, 1974, 81.

Mateo, C.R., Lillo, M.P., Brochon, J.C., Martínez-Ripoll, M., Sanz-Aparicio, J., Acuña, A.U., J. Phys. Chem., 97, 1993, 3486

Memming, R., Z. Physik. Chem., 28, 1961, 169.

Mialocq, J.C., Boyd, A.W., Jaraudias, J., Sutton, J., Chem. Phys. Lett., 37, 1976, 236.

Mialocq, J.C., Goujon, P., Arvis, M., J. Chem. Phys., 76, 1979, 1067.

Minch M.J., Sadiq Shah S., J. Org. Chem., 44, 3252, 1979

Momicchioli, F., Baraldi, I., Berthier, G., Chem. Phys., 123, 1988, 103.

Mulliken, R.S., J. Chem. Phys., 7, 1939, 364.

Nakashima N, Ando R., Fukushima H., Kunitake T., J. Chem. Soc., Chem. Commun., $1982,707$.

Nee, T.W., Zwanzig, R., J Chem. Phys., 52, 1970, 6353.

Negri, R.M., "Procesos Fotofísicos de Ftalocianinas, Porfimeros y Cianinas en Solución", Tesis Doctoral, 1991, Facultad de Ciencias Exactas y Naturales, UBA.

Orlandi, G., Siebrand, W., Chem. Phys. Lett., 30, 1975, 352.

Paladini, A.A., Weber, G., Rev. Sci. Instrum., 52(3), 1981, 419.

Perrin, F., J. Phys Radium, 7, 1926, 390

Perrin, F., Ann. de Phys., 12, 1929, 169.

Perrin, F., Journal de Physique, 5, 1934, 497.

Ponterini, G., Caselli, M., Ber. Bunsenges. Phys. Chem., 96(4), 1992, 564.

Ponterini, G., Momicchioli, F., Chem. Phys, 151, 1991, 111 
Razumova, T.K., Tarnovskii, A. N., Opt. Spectrosc. (Engl. Transl.), 73, 1992, 662.

Richardson, S., J. Fluid Mech., 59, 1973, 707.

Roosbroeck,W., Shockley, W., Phys. Rev., 94, 1954, 1558

Rulliere, C., Chem. Phys. Lett., 46, 1976, 303.

Scaffardi, L., Bilmes, G.M., Schinca, D., Tocho, J.O., Chem. Phys. Lett., 140, 1987, 163

Sension, R.J., Hochstrasser, R.M., J. Chem. Phys., 98, 1993, 2490.

Shank, C.V., Ippen, E.P., Appl. Phys. Lett., 26, 1975, 62

Sheves, M., Friedman, N., Angew. Chem. Int. Ed. English., 25, 1986, 284.

Sibbett, W, Taylor, J.R., Welford, D., IEEE J. Quantum Electron, QE17, 1981, 500.

Smith, D.L., Photogr. Sci. Eng., 18, 1974, 309.

Spears, K.G., Cramer, L.E., Chem. Phys., 30, 1978, 1.

Strickler, S.J., Berg, R.A., J. Chem. Phys., 37, 1962, 814.

Sturmer, D.M., Heseltine, D.W., The Theory of the Photographic Process, 4th Edition, Editor T.H. James. Mc Millan Publishing Co., Inc. New York, 1977.

Sundström, V, Gillbro, T., J. Phys. Chem., 86, 1982, 1788

Tao, T., Biopolymers, 8, 1969, 609

Tocho, J.O., Duchowicz, R., Scaffardi, L., Bilmes, G.M., Di Paolo, R.E., Murphy, M., Trends in Physical Chemistry, 3, 1992, 31

Valdmanis, J.A., Fork, R.L., IEEE J. Quant. Electron., QE22, 1986.

Vaveliuk, P., Scaffardi, L.B., Duchowicz, R., J. Phys. Chem., 100(28), 1996, 11630.

Vaveliuk, $P$., comunicación privada.

Velsko, S.P., Fleming, G.R., Chem. Phys., 65, 1982, 59

Waldeck, D., Cross, A.J., McDonald, D.B., Fleming, G.R., J. Chem. Phys., 74, 1981, 3381

Waldeck, D.H., Fleming, G.R., J. Phys. Chem., 85, 1981, 2614.

Weast, R.C., (editor) Handbook of Chemistry and Physics, 50th Edition (CRC, Boca Raton, 1970)

Weber, G., J. Chem. Phys., 55, 1971, 2399.

Weber, G., Biochem. J., 51, 1952, 145 
West, W., Pearse, S., Grum, F.; J. Phys. Chem., vol.71(5), 1967, 1316.

Wheatley, P.J., J.Chem. Soc., 1959, 4096.

Wiberg, K.B., Physical Organic Chemistry, 2nd Edition, 1966. John Wiley \& Sons, Inc

Winkworth, A.C, Osborne, A.D., Porter, G., Springer Series in Chem. Phys. 23

Picosecond Phenomena III, Ed. F.P. Schäfer, Springer-Verlag Berlin, 1982, pag. 228.

Woessner, D.E., J. Chem. Phys., 37, 1962, 647.

Youngren, G.K., Acrivos, A., J. Chem. Phys., 9, 1975, 3846.

Zechmeister, L., Pinckard, J.H., Experimentia, 9, 1953, 16.

Zwanzig, R., J. Chem. Phys., 68, 1978, 4325. 